\title{
Searching for Low-Mass Dark Matter with SuperCDMS Soudan Detectors
}

\author{
by \\ William Alexander Page \\ B.A., Bowdoin College, 2013 \\ M.Sc., The University of British Columbia, 2015 \\ A THESIS SUBMITTED IN PARTIAL FULFILLMENT OF \\ THE REQUIREMENTS FOR THE DEGREE OF \\ DOCTOR OF PHILOSOPHY \\ in
}

The Faculty of Graduate and Postdoctoral Studies

(Physics)

THE UNIVERSITY OF BRITISH COLUMBIA

(Vancouver)

February 2019

(C) William Alexander Page 2019 
The following individuals certify that they have read, and recommend to the Faculty of Graduate and Postdoctoral Studies for acceptance, the dissertation entitled:

\section{Searching for Low-Mass Dark Matter with SuperCDMS Soudan} Detectors

submitted by William Alexander Page in partial fulfillment of the requirements for the degree of Doctor of Philosophy in Physics.

\section{Examining Committee:}

Scott Oser, Physics and Astronomy

Supervisor

Christopher Hearty, Physics and Astronomy

Supervisory Committee Member

Douglas Bonn, Physics and Astronomy

University Examiner

Jiahua Chen, Statistics

University Examiner

Leslie Rosenberg

External Examiner 


\section{Abstract}

SuperCDMS is a direct-detection dark matter (DM) experiment that uses cryogenically cooled germanium and silicon detectors to search for interactions between DM particles and detector nuclei, and in this thesis I describe my contributions to the experiment.

I start with a brief review of DM and motivate the possibility of its detection in underground laboratories with sensitive detectors, and I review the SuperCDMS detector fundamentals. Then I focus on detector development for the future generation of the experiment, which will deploy an array of detectors at SNOLAB in Sudbury, Canada. Specifically I describe characterization of prototype detectors from surface facility testing, and discuss measurements of critical values that determine the detectors' sensitivity to DM particles, such as the baseline resolution and the phonon collection efficiency. I also describe analysis techniques developed to measure intrinsic detector noise in a high radiation environment such as a surface test facility.

In the final chapters I describe a DM search analysis using four months of data from operation of SuperCDMS detectors in the Soudan Mine in northern Minnesota. I discuss how a particular detector operating mode, called CDMSlite, lowers the energy threshold of the detectors in order to improve the sensitivity to low-mass DM particles. I also present new analysis techniques that optimize the sensitivity to low-mass DM particles, including noise discrimination with multivariate classifiers, instrumental background modeling, and a profile likelihood signal and background fitting approach. In this analysis we set an upper limit on the DM-nucleon scattering cross section in germanium that is a factor of 2.5 improvement over the previous CDMSlite result for a DM mass that is five times the proton rest mass. 


\section{Lay Summary}

It has been well established through astrophysical observations that there is a form of matter that we cannot see ("dark matter") that is five times more abundant than ordinary matter (i.e. stars, planets, dust, etc.) in the universe. Through gravity, dark matter is responsible for the formation of most galaxies, and in that sense we owe our existence to it, but little is known about its character. Leading theories posit that it consists of a halo of particles surrounding most galaxies, and in this model many thousands of dark matter particles stream through the Earth every second with low interaction rates with normal matter. In this thesis I describe my contributions to an experiment designed to detect the rare collisions between dark matter particles and detector material. In an analysis of data from the experiment, I rule out smaller interaction rates between dark matter and these detectors than previous analyses. 


\section{Preface}

Chapter 1 and 2 contain numerous citations to recognize the body of work of others upon which a thesis such as this is built. These contributions include the establishment of the WIMP dark matter paradigm with contributions dating back to the 1920s, as well as the development of CDMS detectors dating back to the early 1990s. These contributions of others are discussed in order to set the stage for the later chapters.

Chapter 3 is based on detector characterization work carried out at the University of California, Berkeley under the supervision of Professor Matt Pyle and research scientist Bruno Serfass. The derivations presented in this chapter resulted from conversations with Matt Pyle. Bruno Serfass assisted greatly with software issues that arose when operating the detectors and analyzing the data. Nicholas Zobrist (now a graduate student at University of California, Santa Barbara) took on the majority of the work required to maintain stable operation of the dilution refrigerator for the 1-2 week intervals during which we tested a series of detectors. The data analysis presented in this chapter is my own.

Chapter 4 and $\mathbf{5}$ are based on an analysis that has been submitted for publication. I was the lead author and responsible for a significant portion of the analysis presented, and co-led the coordination of the analysis effort with D'Ann Barker. We co-chaired weekly working group meetings. Major analysis contributions came from D'Ann Barker and Ryan Underwood. The "Background Models" section (Sec. 5.1) is largely adapted from the manuscript and is based on work carried out by D'Ann Barker. Ryan Underwood carried out the majority of the work in the "Fiducial Volume" section, with conceptual contributions from Wolfgang Rau and some technical contributions from myself. Eleanor Fascione, Dan Jardin, and Andrew Scarff also contributed to the data analysis. Rob Calkins, Scott Oser, and Wolfgang Rau supervised the analysis. As the lead author I contributed significantly to the manuscript composition and ushered it through internal collaboration review as well as through journal peer review. 


\section{Table of Contents}

Abstract $\ldots \ldots \ldots \ldots \ldots \ldots$ iii

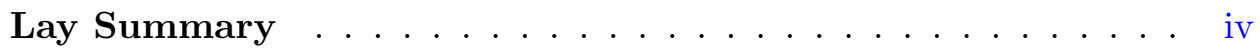

Preface $\ldots \ldots \ldots \ldots \ldots \ldots \ldots \ldots$

Table of Contents .................... . . vi

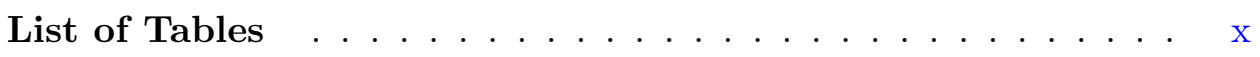

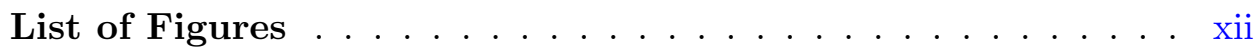

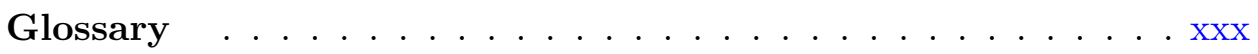

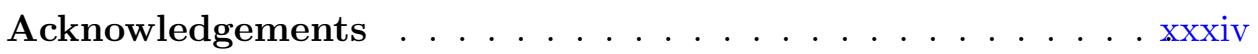

1 Detection of Particle Dark Matter . . . . . . . . . . . . 1

1.1 Early Evidence . . . . . . . . . . . . . . . . 1

1.2 Galaxy Clusters . . . . . . . . . . . . . . . . . . 3

1.3 Big Bang Nucleosynthesis . . . . . . . . . . . . 5

1.4 The Cosmic Microwave Background . . . . . . . . . . 7

1.5 Composition Hypotheses . . . . . . . . . . . . . . . . 8

1.5.1 The WIMP Hypothesis . . . . . . . . . . . . 9

1.5 .2 Axions .................... 11

1.5.3 Dark Sector Particles . . . . . . . . . . . . . 11

1.6 Dark Matter Detection . . . . . . . . . . . . . . 12

1.6.1 Direct Detection . . . . . . . . . . . . 12

1.6.2 Collider Production . . . . . . . . . . . . . 18

1.6.3 Fixed Target . . . . . . . . . . . . . . . 19

1.6.4 Indirect Detection . . . . . . . . . . . . . . 20

1.7 Further Reading . . . . . . . . . . . . . . . . 21 
2 Detectors for the Cryogenic Dark Matter Search . . . . . 22

2.1 Semiconductor Detector Physics . . . . . . . . . . . . 22

2.1.1 Electron Recoils . . . . . . . . . . . . . . 23

2.1.2 Nuclear Recoils . . . . . . . . . . . . . . . . 24

2.1.3 Yield Discrimination . . . . . . . . . . 25

2.1.4 Phonon Generation and Propagation . . . . . . . 26

2.2 Amplifiers . . . . . . . . . . . . . . . . 29

2.2.1 Measuring the $e^{-} / h^{+}$Energy . . . . . . . . . . 31

2.2.2 Measuring the Phonon Energy . . . . . . . . . . 32

2.3 Optimal Filter Event Reconstruction _. . . . . . . . . . 33

2.3.1 Simple 1D Optimal Filter . . . . . . . . . . . . 34

2.3.2 Non-Stationary Optimal Filter . . . . . . . . . . 36

2.3.3 Two Template Optimal Filter . . . . . . . . . 38

2.3.4 Use of Different OF Algorithms . . . . . . . . . 38

2.4 iZIP Interleaved Design _ . . . . . . . . . . . 40

2.5 CDMSlite Detectors . . . . . . . . . . . . . . . . 42

2.5.1 CDMSlite Biasing and Readout Configuration . . . . 44

2.5.2 Calibration ................... 45

2.5.3 CDMSlite History: Run 1 and Run $2 \ldots \ldots$

3 Detector Characterization . . . . . . . . . . 55

3.1 Detector Resolution . . . . . . . . . . . . . 55

3.1.1 Data-Driven Resolution Measurement . . . . . . . 56

3.1 .2 Phonon Amplifier Noise . . . . . . . . . . . . . 59

3.1 .3 TES Voltage Biasing . . . . . . . . . . 60

3.1.4 Phonon Collection Efficiency . . . . . . . . . . . . 62

3.1.5 Optimal Filter Baseline Resolution . . . . . . . . 66

3.2 Detector Leakage Current . . . . . . . . . . . . . . . 67

3.2.1 Detector Ionization Environment at Surface Facilities 69

3.2.2 Muon Veto Analysis Techniques . . . . . . . . . . 70

3.2.3 Phonon Noise as a Function of Detector Voltage . . . 73

4 Data Analysis of CDMSlite Run 3 f . . . . . . . . . . 85

4.1 Motivation .................. . . 85

4.2 Salting . . . . . . . . . . . . . . 86

4.2.1 Determining the Target Number of Salt Events . . 86

4.2.2 Selecting Events To Replace . . . . . . . . . . . 88

4.2.3 CDMSlite R3 Salt Application and "Unsalting" . . . 89

4.3 Experimental Setup . . . . . . . . . . . . . . . . 90

4.3.1 Detector Selection and Configuration . . . . . . 90 
4.3.2 Vibration Monitoring . . . . . . . . . . . . 91

4.3.3 High Voltage Current Monitoring . . . . . . . . . 93

4.3.4 Charge Triggers . . . . . . . . . . . . . . . 94

4.4 Energy Scale . . . . . . . . . . . . . . . . . . 96

4.4.1 Energy Corrections . . . . . . . . . . . . . . 96

4.4.2 Nuclear Recoil Energy Scale . . . . . . . . . . . 100

4.5 Quality Cuts ................... . . 102

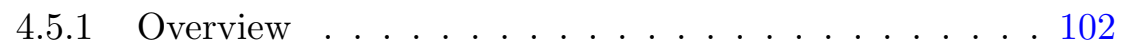

4.5.2 Prepulse Noise Cut . . . . . . . . . . . . . 103

4.5.3 Bad Series Cut . . . . . . . . . . . . . . . 104

4.5.4 Phonon Pulse-Shape Cuts . . . . . . . . . . . . 104

4.6 Low Frequency Noise Cuts . . . . . . . . . . . . . . . . . 112

4.6 .1 Motivation . . . . . . . . . . . . . 112

4.6.2 High Energy LF Noise Cut . . . . . . . . . . . . . . . 113

4.6.3 Detector-Detector Correlations . . . . . . . . . . . 114

4.6.4 Motivation for Multiple Templates . . . . . . . . . . 116

4.6.5 Boosted Decision Tree (BDT) . . . . . . . . . . 119

4.7 Bifurcated Analysis . . . . . . . . . . . . . . . 121

4.7 .1 Introduction . . . . . . . . . . . . 123

4.7.2 Application to CDMSlite Run $3 \ldots \ldots$. . . . . . 125

4.7.3 LF Noise Background Correlation . . . . . . . . . . . 127

4.7 .4 Box Relaxation . . . . . . . . . . . . . . 132

4.8 Fiducial Volume . . . . . . . . . . . . . . . . . . . . . 132

4.8.1 Radial Pulse Simulation . . . . . . . . . . . 135

4.8.2 Energy Distribution of Reduced NTL events . . . . . 136

4.8.3 Radial Distribution of Reduced NTL events . . . . . 137

4.8.4 Optimizing the Radial Cut . . . . . . . . . 137

4.9 Livetime and Signal Efficiency . . . . . . . . . . . . . . . . 138

4.9 .1 Livetime . . . . . . . . . . . . . . . . 139

4.9 .2 Trigger Efficiency . . . . . . . . . . . . . . . 140

4.9 .3 Signal Simulation . . . . . . . . . . . . . . . 142

4.9 .4 Quality Cut Efficiency . . . . . . . . . . . 144

4.9.5 Fiducial Volume Efficiency . . . . . . . . . . . . 146

4.9.6 Combining and Parametrizing the Efficiency . . . . 147

\section{Profile Likelihood Dark Matter Search with CDMSlite Run}

3 . . . . . . . . . . . . . . . . . . . . . 149

5.1 Background Models . . . . . . . . . . . . . . . . 149

5.1.1 Energy Resolution Model . . . . . . . . . . . . 150

5.1 .2 Cosmogenic Activation . . . . . . . . . . . 152 
5.1.3 Electron Capture of ${ }^{71} \mathrm{Ge} \ldots \ldots . . . \ldots 154$

5.1 .4 Compton Scattering . . . . . . . . . . . 155

5.1 .5 Surface Backgrounds . . . . . . . . . 156

5.2 Likelihood and Limit Setting . . . . . . . . . . . . . . . . 158

5.2.1 Components Needed for the Likelihood . . . . . . . . 158

5.2 .2 Likelihood Function . . . . . . . . . . . . . . 162

5.2 .3 Limit-Setting Approach . . . . . . . . . . . . . . 165

5.3 Results . . . . . . . . . . . . . . . . . . . 168

5.3.1 Background Model Goodness of Fit . . . . . . . 168

5.3 .2 Exposure of Salt . . . . . . . . . . . . . . 170

5.3 .3 Final Spectrum Fit . . . . . . . . . . . . . 171

5.3 .4 Background Rates . . . . . . . . . . . . . . . . 172

5.3 .5 Sensitivity Estimate . . . . . . . . . . . . . 175

5.3 .6 DM Limit . . . . . . . . . . . . . . . 176

6 Conclusion and Future Outlook . . . . . . . . . . 178

Bibliography . . . . . . . . . . . . . . . 181

\section{Appendices}

A Voltage Scan Calibration Results . . . . . . . . . . . . 211

A.1 Linearity . . . . . . . . . . . . . . . . . . . . 211

A.2 Significance of Calibration Systematics . . . . . . . . . 211 


\section{List of Tables}

2.1 Electron-hole pair creation energies (in eV) and bandgap energies for germanium and silicon. Values from Ref. [62]. . . . 23

2.2 Detector amplifier specifications for different generations of the germanium CDMS detectors. Adapted partially from Ref. [66]. . . . . . . . . . . . . . . 31

2.3 Important time and frequency values for charge $\left(e^{-} / h^{+}\right)$and phonon amplifier digitizers from SuperCDMS Soudan. $R, N$, $T, \Delta f, \Delta t$ refer respectively to the digitization rate, the number of bins in the read out time stream, the time length of the read out time stream, the lowest resolvable frequency $(1 / T)$, and the time length of one digitization bin. . . . . . . . 34

2.4 The primary use of the three different OF algorithms. . . . . 40

2.5 The energies and probabilities of electron capture for ${ }^{71} \mathrm{Ge}$. The $N$-shell is included, though events from this EC have never been observed in the CDMSlite detectors because of the low energy of this process. . . . . . . . . . 46

3.1 Measured resistances and bias currents $\left(\mathrm{I}_{b}\right)$ by channel on the S12C detector.) The values below the double horizontal line are not directly measured, but rather derived based on the schematics of Fig. 3.4, where $V_{b}=I_{b} R_{s}, R_{l}=R_{p}+R_{s}$, and the shunt resistor $R_{s}$ is taken to be $0.024 \Omega$ based on historical measurements. . . . . . . . . . . . . 65

3.2 The leakage current, as well as the effective total ohmic detector resistance, derived from the listed excess noise levels at low frequency on the $\mathrm{S} 12 \mathrm{C}$ detector. . . . . . . . . . 81

5.1 Background components considered in the likelihood fit . . . 151

5.2 Reconstructed energies and resolutions of the ${ }^{71} \mathrm{Ge}$ decay peaks and the baseline noise in CDMSlite Run 3. . . . . . . . 152

5.3 Best-fit energy resolution parameters of the model in Eq. 5.2 for Period 1 and Period 2. . . . . . . . . . . 152 
5.4 Cosmogenic isotopes that decay via electron capture and are present in the measured CDMSlite spectrum. The shell energies $\mu$, given in $\mathrm{keV}$, are from Ref. [130]. The amplitudes $\Lambda$, from Ref. [131], are normalized with respect to the $K$ shell. . 154

5.5 Compton model parameters for CDMSlite, normalized over the energy range 0-20 keV. All values have been multiplied by a factor of $10^{3}$ and are in units of $\mathrm{keV}^{-1}$. . . . . . . . 156

5.6 Constrained or bound variables in the likelihood fit . . . . . . 165

5.7 Average single-scatter event rates for energy regions between the activation lines in Run 2 and Run 3, corrected for efficiency. All errors contain $\pm \sqrt{N}$ Poissonian uncertainties, and the lowest energy range values additionally include uncertainty from the signal efficiency. . . . . . . . . . . . 175

6.1 The projected exposures and detector parameters for the four types of SNOLAB detectors: Ge iZIP, Si iZIP, Ge HV, Si HV. The exposures are based on 5 years of operation with $80 \%$ live time. The HV detectors do not have ionization sensors. The arrangement will be 4 towers of 6 detectors each. Table reproduced from Ref. [97]. . . . . . . . . . . . . . 180 


\section{List of Figures}

1.1 (left) The hydrogen surface densities (azimuthally averaged) for five galaxies and (right) the rotation galactic rotation curves. The bars under the galaxy names indicate the spatial resolution of the measurements. $\mathrm{R}_{80}$ corresponds to the galactic radius containing $80 \%$ of the Hydrogen density determined from the $21 \mathrm{~cm}$ emission. Plot copied from Ref. [5], with permission. Measurements were performed by Rogstad and Shostak in 1972 from the Owens Valley Radio Observatory using $21 \mathrm{~cm}$ observations. . . . . . . . . . . . . . .

1.2 1E 0657-56 (C) 2004 X-ray: NASA/CXC/CfA/M.Markevitch et al.; Optical: NASA/STScI; Magellan/ U.Arizona/D.Clowe et al.; Lensing Map: NASA/STScI; ESO WFI; Magellan/ U.Arizona/ D.Clowe et al., by permission) [11] [12] . . . . . .

$1.3 Y$ is the ${ }^{4} \mathrm{He}$ mass fraction of all baryons. The blue, red, and green bands are the modeled number densities of ${ }^{2} \mathrm{H},{ }^{3} \mathrm{He}$, and ${ }^{7} \mathrm{Li}$, respectively, relative to ${ }^{1} \mathrm{H}$ as a function the baryon density. Yellow boxes show measurements (95\% CL) of the light element abundances; of note is that only upper limits on ${ }^{3} \mathrm{He}$ abundance have been made, and the measurements of ${ }^{7} \mathrm{Li}$ are in slight tension with the model. The pink hatched band shows the inferred baryon to photon ration from the ${ }^{2} \mathrm{H}$ measurement, while the blue hatched band shows the range indicated by CMB measurements. From [14] with permission.

1.4 CMB power spectrum predicted by a $\Lambda$-CDM cosmology (i.e. a dark energy (69\%) and cold dark matter (26\%) dominated universe). Data points in red are measurements by the Planck collaboration [15]. Power spectrum of temperature fluctuations in the Cosmic Microwave Background (C) 2013 ESA/Planck, and the Planck collaboration, from Planck 2013 results. I. Overview of products and results, by permission). . . . . . 7 
1.5 Number density of WIMPs in the Universe as a function of time, where the relic density depends on the WIMP annihilation cross section, $\sigma_{\chi \bar{\chi}}$ (C) NASA/IPAC Extragalactic Database (NED) which is operated by the Jet Propulsion Laboratory, California Institute of Technology, under contract with the National Aeronautics and Space Administration, by permission). . . . . . . . . . . . . . . 10

1.6 Current (solid) and projected (dashed) $90 \%$ limits on the WIMP mass vs. WIMP-nucleon cross section parameter space. The yellow shaded region represents the DM cross section at which experiments will observe an "irreducible" background from nuclear scattering neutrino events. At lower DM mass, the background from solar neutrinos from ${ }^{7} \mathrm{Be}$ and ${ }^{8} \mathrm{~B}$ reactions dominate, whereas at higher DM mass atmospheric and the diffuse supernovae neutrino background (DSNB) dominate. Figure from SuperCDMS collaboration approved public plots. . . . . . . . . . . . . . . . 16

1.7 The expected WIMP event rate for the given $m_{\chi}$ and spinindependent cross section. $\sigma_{S I}=10^{-41} \mathrm{~cm}^{2}$ corresponds to roughly the cross section reported by DAMA/LIBRA, CRESST, CDMS $\mathrm{Si}$, and CoGent. $\sigma_{S I}=10^{-45} \mathrm{~cm}^{2}$ corresponds to a cross section excluded only by results in the last $2-3$ years. Internal CDMS figure, from [46]. . . . . . . . . . . . . 17

1.8 The depth of different underground sites, shown as muon flux on the y-axis, and meters of water equivalent (mwe) on the $\mathrm{x}$-axis, from different generations of CDMS experiment. SUF stands for Stanford Underground Facility. . . . . . . . . . 18

1.9 Limits on the DM-nucleon scattering cross section, as a function of DM mass from the CMS (left) and ATLAS (right) experiments for different sets of model assumptions. Figures from $[47,48] \ldots \ldots \ldots \ldots \ldots$

2.1 Estimated energy vs. electron range in germanium and silicon, based on the electron stopping power from Coulomb interactions. Figure adapted from [61]. . . . . . . . . . 24

2.2 Measurements of the ionization yield of nuclear recoil events, as a function of energy. The prediction from the Lindhard model is overlaid. From Ref. [73]. . . . . . . . . . . 26 
2.3 Ionization yield versus recoil energy for calibration data, from a CDMS II detector. The blue data was acquired during exposure of the detector to a neutron ${ }^{252} \mathrm{Cf}$ source producing primarily NRs, which accordingly have a measured ionization yield less than 1 . The red data is from a ${ }^{133} \mathrm{Ba}$ calibration, which primarily consists of ERs from $\gamma$ interactions in the detector. The black bands correspond to the $\pm 2 \sigma$ area in the ionization yield vs. recoil energy plane where ERs and NRs occur. These bands provide a visualization of the discrimination in this plane, and a cut in this plane would be customized for a specific analysis (not necessarily at the $\pm 2 \sigma$ level). From Ref. [46]. . . . . . . . . . . . . . . . 27

2.4 A simple depiction of an event in the detector and the resulting prompt, NTL (or Luke), and recombination phonon production. . . . . . . . . . . . . . 27

2.5 (top) A cartoon of the iZIP detector, with the individual phonon channels labeled and uniquely colored. (bottom) A characteristic phonon signal (i.e. pulse) from the bulk of an iZIP detector. The different pulse shapes on the individual channels indicate that this event occurred close to channel DS2 and CS1. . . . . . . . . . . . . . . 30

2.6 (left) An example simulated pulse with the template (normalized to the best fit amplitude as given by the optimal fit). The template is also left-right aligned with the best fit time delay as given by the optimal fit. (right) The frequency domain representation (magnitude only) of the template, noise (PSD), and optimal filter.

2.7 (left) Example $10 \mathrm{keV}$ total phonon pulses with the OF template overlaid in blue. (right) The residuals (phonon pulse - template) showing that the position-dependent part of the pulse can be treated as a non-stationary source of noise. . . 37

2.8 (top)An example of the 2 template fit to data, where the prompt signal is modeled by the fast template and the ballistic signal is modeled by the slow template. (bottom) An example of the 2 template fit performed on the 4 individual phonon channels for a single event on a CDMSlite detector. . . . . . 39

2.9 The iZIP design with interleaved charge electrodes $( \pm 2 \mathrm{~V})$ and phonon rails (0V). Used with permission, from [81]. . . . . 40 
2.10 (left) The electric field and potential lines produced from the phonon rails (yellow) and charge electrodes (green). Notice that the unique surface E-field extends $\sim 1 \mathrm{~mm}$ into the crystal and therefore surface events within this margin should exhibit asymmetric charge collection. (right) Data from iZIP detector T3Z1 showing surface event discrimination (discussion in main text). Internal CDMS figure, used with permission, from [81]. . . . . . . . . . . . . . . . . . . . . 41

2.11 (left) The iZIP phonon channel partition on a single side, with channel names labeled. (right) The px-py partition for uniformly distributed events for an iZIP detector, where the triangular shape is due to the channel layout as well as the partition variable definition (Eq. 2.17, 2.19). . . . . . . . 43

2.12 A rough schematic of the custom CDMSlite biasing electronics, which shows that only a single side of the detector is read out while the other side is biased using the "High Voltage" power supply (credit: R. Thakur [83]). . . . . . . . . .

2.13 DM recoil spectra for different DM masses. The vertical lines show example energy thresholds and demonstrate the importance of threshold for sensitivity to a low mass DM-nuclear recoil scattering. The CDMSlite detectors have achieved thresholds of $\sim 0.5 \mathrm{keV}_{\mathrm{nr}}$. (credit: M. Pepin [84]) . . . . . . . .

2.14 The reconstructed total phonon energy of data with ${ }^{71} \mathrm{Ge}$ electron capture peaks present. The colors label data taken at different detector voltages, where the NTL amplification is apparent at the $30 \mathrm{~V}, 50 \mathrm{~V}$, and $70 \mathrm{~V}$ data. The energy scale of the data at each voltage is calibrated so that the $K$-shell peaks appear at the correct energy (i.e. the total phonon energy given by Eq. 2.9), and additional details and insights gained from these "voltage scan" data are discussed in Sec. A.1. . . 47 
2.15 (top) A simple depiction of a regular event from a particle interaction alongside two leakage events, one from the detector bulk and one at the detector interface. (middle) From P. Luke's publication, showing the signal-to-noise benefits of NTL amplification up to $\sim 140 \mathrm{~V}$ for the diode detector described in Ref. [76]. (bottom) The baseline total phonon noise RMS, labeled as " $\sigma_{O F}\left[\mathrm{keV}_{\mathrm{t}}\right]$ " for the T5Z2 detector, showing an increase in the noise above $\sim 60 \mathrm{~V}$ and a severe worsening of the noise above $\sim 70 \mathrm{~V}$. The triangles on this plot provide supplemental measurements of the noise RMS without pre-biasing, demonstrating the effectiveness of the pre-bias procedure. . . . . . . . . . . . . . . 49

2.16 A simple schematic of the CDMSlite biasing circuit. . . . . . 51

2.17 The CDMSlite Run 2 energy spectrum after all cuts have been applied, with the energy of the events measured by the nonstationary optimal filter, and converted to the nuclear recoil energy using a Lindhard $k$ parameter of 0.159 . One of the energy intervals is highlighted between events as a visualization of the energy intervals considered by the optimum interval method. The optimal interval considers intervals between all pairs of events (not necessarily adjacent pairs of events) . . . 53

2.18 Spin-Independent WIMP-nucleon cross section 90\% CL upper limits from Run 1 and Run 2 (black solid with 95\% uncertainty band) compared to the other most sensitive results in this mass range (at the time of publication of the Run 2 result): CRESST [94] below 1.6 $\mathrm{GeV} / c^{2}$ and PandaX-II [43] above $4 \mathrm{GeV} / c^{2} \ldots \ldots \ldots \ldots \ldots . \ldots \ldots$

3.1 (left) Example simulated random trigger (noise only) with the best fit OF template overlaid in red. Even though there is zero signal, noise fluctuations result in the OF algorithm fitting a small non-zero amplitude. (right) The OF amplitude fit results (from a detector with two readout channels) of many random triggers, when the $\mathrm{OF}$ algorithm has no constraint on the time delay search window [96]. . . . . . . . . . 57

3.2 (left) Data from ${ }^{133} \mathrm{Ba}$ calibration of the $\mathrm{S} 12 \mathrm{C}$ detector, in the total phonon integral vs. total phonon OF energy estimates. (right) The S12C detector baseline resolution measurement using random triggers and measured using the constrained time-offset $\mathrm{OF}$, giving a resolution of $52.4 \mathrm{eV} . \ldots \ldots 59$ 
3.3 (top) The current noise (amps $/ \sqrt{\mathrm{Hz}}$ ) for each channel, the total phonon channel sum, and the quadrature channel sum for the S12C iZIP detector. (bottom) The S12C power noise (watts $/ \sqrt{\mathrm{Hz}}$ ), converted from the measured current noise using the $(\mathrm{d} I / \mathrm{d} P)$ conversion described in Sec. 3.1.4. . . . . . 61

3.4 (left) Simplified circuit diagram for the TES amplifier, with the shunt resistor $\left(R_{s}\right)$, parasitic resistor $\left(R_{p}\right)$, inductor $(L)$, and TES element labeled. (right) The Thevenin equivalent voltage-biased TES circuit, where $V_{b}=I_{b} \cdot R_{s}$ and the load resistor is given by $R_{l}=R_{p}+R_{s} . \ldots \ldots \ldots$. . . . . 61

3.5 The simplified TES thermal diagram. In this simplified model, the bath represents the collection of the crystal and the dilution refrigerator. More realistic thermal models are considered in Ref. $[77,95] . \ldots \ldots \ldots . \ldots . \ldots 63$

3.6 (left) The phonon collection efficiency $\left(\epsilon_{\text {phonon collection }}\right)$ calculated for events in the $100-200 \mathrm{keV}$ range, where $E_{\text {collected }}$ is calculated using the total phonon signal (the channel sum). The solid (dashed) histogram represents the $E_{\text {true }}$ measured by the optimal filter (pulse integral) energy estimate. (right) The phonon collection efficiency measured on a channel-bychannel basis. The individual $\mathrm{S} 12 \mathrm{C}$ channels (of which there are 8 total) on average collect $2-3 \%$ of the event's energy. . . 66

3.7 The power noise observed on S12C with channel Cs2 removed, with the detector baseline resolution (from Eq. 3.16) provided in the overlaid text. . . . . . . . . . . . .

3.8 A simulated representation of the energy deposition in surface detectors with iZIP dimensions (76mm diameter) as well as for larger diameter $(100 \mathrm{~mm})$ detectors being fabricated for the SNOLAB experiment. The time window over which an event is typically digitized $(50 \mathrm{~ms})$ is given by the time window shown by the vertical lines around 13.5 sec. . . . . . . . . 69

3.9 Random traces acquired on a prototype iZIP detector operated at a surface test facility, showing the slopes due to muon "tails" evident to varying degrees on top of the underlying phonon noise. The labels in the top left of the plots correspond to the labels in scatter plot shown in Fig. 3.11. . . . .

3.10 The PSD constructed with slopeless random traces (labeled as "underlying noise"). The dashed PSDs are calculated with random triggers with varying steepness of slopes (in units of amps $/ \mathrm{ms}$ ) in the randoms. . . . . . . . . . 72 
3.11 (top) The distribution of randoms in the DC value vs slope plane, showing the approximate acceptance region of this parameter space to select the random traces that are representative of the underlying TES noise. The red symbols highlight the region in this plane that the raw traces in Fig. 3.9 are selected. (bottom) A projection of the data onto just the trace slope axis, showing that the selection criteria become increasingly inefficient when the detector is operated at high voltage. . . . . . . . . . . . . . . .

3.12 Examples of time domain noise from an iZIP-style prototype detector. The data come from two channels: channel A, top, blue and channel B, bottom, orange. The left (right) panel show noise traces from these two channels, with the detector at $0 \mathrm{~V}(44 \mathrm{~V})$. The traces read out at $44 \mathrm{~V}$ clearly have worse noise. It is also clear that the excess noise is correlated between channels, which is expected from a charge leakage noise source that stochastically generates NTL phonons distributed as small phonon pulses. These small pulses are absorbed equivalently to the standard phonon pulses on the iZIP - equally on all channels in the ballistic limit - which leads to correlated noise. . . . . . . . . . . . . .

3.13 (top) Power Noise PSDs measured on a phonon channel on the S12C detector (with negative HV applied to side 2, reading out side 1). Overlaid on each PSD is a leakage current model using the measured phonon fall time. (bottom) Power Noise PSDs measured on a phonon channel of the G23R prototype detector. . . . . . . . . . . . . .

3.14 The black data depicts a time series of the shot noise model, where single charge quanta are distributed with inter-arrival times randomly sampled from a $e^{-\lambda t}$ distribution (i.e. a Poisson process). For visualization purposes the delta functions have been widened from being infinitely narrow to $0.5 \mathrm{~ms}$ wide. The average current used in this simulation was $20 \times 10^{-18}$ amps (corresponding to an average of 6.25 charge quanta per $50 \mathrm{~ms}$ interval, which fluctuated to 8 charges in this realization). The blue data depicts the phonon power generated by the charges traversing the detector, for a $50 \mathrm{~V}$ detector bias, and for a phonon absorption time of $\tau_{F}=175 \mu$ s to match the $\mathrm{S} 12 \mathrm{C}$ detector. . . . . . . . . . . . . . . 77 
3.15 Example of the simulation of a single $50 \mathrm{~ms}$ data stream of charge leakage shot noise injected onto fundamental TES noise. The green pulse is amplified by a factor of 10 (in order to be visible) and its fall time is $\tau_{f}=175 \mu$ s to match the S12C detector. Many of the pulses are Poisson distributed in time and added to the fundamental TES noise, giving the blue curve. . . . . . . . . . . . . . .

3.16 PSDs are made of the simulated traces using different detector voltages in the simulations. The noise shape and magnitude are consistent with those predicted by the shot noise model (Eq. 3.23), as well as consistent with the noise shape seen in the data (e.g. Fig. 3.13). . . . . . . . . . . . . . . .

3.17 Leakage current measurements, as a function of voltage, for a number of detectors (primarily prototype, primarily iZIP style and size). All detectors here were measured at a surface test facility, with the exception of the blue "T5Z2" data point. This data point is determined using data acquired from Soudan, but is a rough estimate for a number of reasons, one of which is that low frequency noise present in all PSDs from Soudan masked the shot noise signal. . . . . . . . . . . 82

3.18 (left) The total phonon baseline resolution $\left(\sigma_{\mathrm{t}}\right)$ for a number of detectors as a function of detector voltage. The solid lines express ideal (no leakage current) resolution scalings as a function of voltage where the $\sigma_{\mathrm{t}}$ value remains constant as a function of voltage. The data points represent resolution measurements at those voltages, or deduced resolutions based on PSD measurements and Eq. 3.16. (right) The electron equivalent energy resolution $\left(\sigma_{\mathrm{t}}\right)$ as a function of voltage, derived from the left hand plot using Eq. 3.26. . . . . . . . . . . 83

4.1 (left) The CDMSlite Run 2 90\% CL OI exclusion spin- independent cross section limit as a function of DM mass. (right) The number of DM events that correspond to the excluded $\sigma_{S I}$ from the left figure. . . . . . . . . . . . . 87

4.2 Example "salt" added to the CDMSlite Run 2 final spectrum at the magnitude indicated by the excluded 90\% CL for a DM signal. The salt spectrum is scaled to a much higher level than the exclusion limit to illustrate its shape. . . . . . 88 
4.3 The distribution of ${ }^{252} \mathrm{Cf}$ calibration data in the crycooler vibrational phase variable (labeled as CryoPreTime Mod 0.83s). As the selection criteria moves to higher energy, the correlation of events with the phase decreases. . . . . . . . . . 90

4.4 (left) A depiction of the SuperCDMS Soudan detector array, with 15 iZIPs total arranged in 5 "towers." (right) A depiction of an iZIP detector read out in CDMSlite mode. Copied from Ref. [82], with permission. . . . . . . . . . . . . 91

4.5 The low energy event density dependence on the the cryocooler vibrational phase is shown in the red histogram. Good events from actual energy depositions, shown in the blue histogram, are not correlated with the vibrational phase. . . . . 92

4.6 An event density plot of lower energy events $\left(-2<\mathrm{keV}_{\mathrm{t}}<\right.$ $5)$ that are more likely to be instrumental noise events than events from particle energy depositions in the detector. Ba and Cf calibrations are highlighted. A Cf period when no data was read out is between May 01 and May 05. The April 14 stripe is from a single series (01150414_1430) when the cold trap was being cleaned. A second 3 hour series on May 05 (01150505_1422) was removed from the plot as it was saturating the color map - this was the first series after the late-Run Cf calibration. . . . . . . . . . . . . . . 93

4.7 The RF room temperature (TE_FEB_RACK) $\left({ }^{\circ} \mathrm{F}\right)$ and the leakage current (iseg_hv_current_b) (nA) as a function of calendar time. The x-axis runs from mid-February to early April, 2015. . . . . . . . . . . . . . . . 94

4.8 Correlation between trigger rate and detector voltage. The red curve is the best fit of the trigger rate to the detector voltage model of Eq. 4.2. . . . . . . . . . . . . . . . 94

4.9 Representative PSDs from R3a (left) and R3b (right). The colored curves represent the noise on the individual channels, while the black curve represents the channel sum. . . . . . 96

4.10 The drift in the total phonon energy of the $10 \mathrm{keV}$ line, with the HV current correction prediction overlaid. The other energy scale corrections have been applied to the data in order to highlight the corrections from the HV current. . . . . . . 97 
4.11 The steps of the linear base temperature correction. After fitting a line in the $E_{c o r r}$ (y axis) vs. base temperature (x axis) plane, the linear dependence is removed to form a corrected $E_{\text {corr } 3}$ variable. A Gaussian fit of the $K$-shell events' energies (as measured by $E_{\text {corr } 3}$ ) shows the energy resolution. . . . . . 98

4.12 Data from single-sided readout of T5Z2 at 0V. (left) Raw total phonon traces plotted from $10 \mathrm{keV}$ events. These events all have the same energy (see alignment in the tail of the pulse), and the dark green-blue traces are selected by their peakiness. (right) The high energy $0 \mathrm{~V}$ spectrum asymmetry in the $10 \mathrm{keV}$ line, with the peakier $10 \mathrm{keV}$ events highlighted in cyan. . . . . . . . . . . . . . . . .

4.13 The steps of the linear 2-template correction. After fitting a line in the $E_{\text {corr } 3}$ (y axis) vs. 2-template fast amplitude ( $\mathrm{x}$ axis) plane, the linear dependence is removed to form a corrected ptNF variable. A Gaussian fit of the $K$-shell in the corrected $E_{\text {corr } 4}$ variable shows improved energy resolution. . 100

4.14 (left) Comparison of the correction factor for $Y=1$ and $Y=$ 0.15 events. Note that the difference in the correction factors is very small for small $I_{\mathrm{HV}}$, but for Run 3, with a leakage current up to $I_{\mathrm{HV}}=120 \mathrm{nA}$, it is clear that we should include this correction. (right) The maximum \% error on a NR energy estimate $\left(E_{n r}\right)$, if the total phonon energy $\left(E_{p t}\right)$ is corrected without consideration of the ionization yield, which results in the nuclear recoil energy incorrectly set higher than what it would be when properly keeping track of the ionization yield in the HV current correction. The $k$ is the Lindhard $k$ parameter for the yield. . . . . . . . . . . . . . 101

4.15 The energy scale conversions for Run 3. The differences from the Run 2 energy scalings, which are negligible only for small HV leakage current, are circled in red. . . . . . . . . . . 102

4.16 (left) The distribution of the prepulse standard deviation of the randoms for a single series, with a Gaussian fit to the distribution overlaid. (right) The trend of fitted Gaussian $\sigma$ for all Run 3 series. . . . . . . . . . . . . . . . . . . . 104

4.17 Comparison of glitch and square templates with the good phonon template. While the square template only roughly resembles a "square" shape, we refer to it as square because it is different from the glitch pulse primarily in that it plateaus for $300 \mu \mathrm{s}$, which it makes it wider. . . . . . . . . . 105 
4.18 (left) An example of a glitch event with the best fits of the good event and glitch template to the event overlaid. (right) An example of a LF noise event with the best fits of the good event and LF noise template to the event overlaid. . . . . . . 106

4.19 (left) The distribution of good events in the $\Delta \chi_{\text {glitch }}^{2}$ vs. ptOF plane, and (right) the same data reprocessed with a single noise PSD, showing that the variations are due to the PSDs that change for each series. . . . . . . . . . . 107

4.20 (top) The $\Delta \chi_{\mathrm{LF}}^{2}$ vs. ptOF plane for a portion of the Run 3 data, showing a high concentration of LF noise events. Raw pulse inspection revealed that only a small fraction of data points in this plot (those data point at the lowest $\Delta \chi_{\mathrm{LF}}^{2}$ values) resembled good events. The $\Delta \chi_{\mathrm{LF}}^{2}$ parameter is calculated with "template 1" as shown in Fig. 4.26. (bottom) The parabolic fit coefficient in the ptOF- $\Delta \chi_{\mathrm{LF}}^{2}$ plane vs. $3.5 \sigma$ contour area, with the clusters shown. . . . . . . . . . 108

4.21 (top) The maximum likelihood estimate best fit of 2 Gaussians plus a flat distribution to noise events in a single series. (bottom) The 9 cryocooler "loud" clusters, and 8 cryocooler "quiet" cluster, all highlighted by time. . . . . . . . . . 110

4.22 (top) A raw trace for a glitch-like event that fails the cut. (bottom) The tuning of the parabolic portion of the cut based upon the parabola of good events, for series block 15. . . . . 111

4.23 (left) The total phonon pulse of the square pulses. (right) the $\Delta \chi_{\text {square }}^{2}$ parameter formed from the square template. . . . . 112

4.24 The simulated signal (blue data points) significantly overlaps the LF noise background (the majority of the red data points) at low energy in this variable plane. . . . . . . . . . . . 114

4.25 (left) Example traces showing detector correlated LF noise on the three Tower 2 detectors (top:T2Z1, middle: T2Z2, bottom: T2Z3). (right) Comparisons of signal and background distributions in the T2Z1-T2Z2 detector trace correlation parameter. . . . . . . . . . . . . . . . 115

4.26 (top) Three different LFN templates used to fit different low frequency noise shapes on T2Z1. (middle) The averaged amplitude of the Fourier transform of the LFN traces. In the red curve, the time domain traces had zeros added to the front and end of the trace before the Fourier transform, which is a trick to to improve the $\delta f$ resolution of the Fourier transform. (bottom) The phase of the black LF noise template. . . . . . 117 
4.27 (left) The $\Delta \chi_{\mathrm{LF}}^{2}$ vs. ptOF plane for the data, shown in red, and the simulated signal, shown in blue, when using template \#1 to fit the raw traces. (right) equivalent to figure left but using template \#2, showing improved separation between the data (which consists primarily of LF noise events), and the signal. . . . . . . . . . . . . . . . . . 118

4.28 The average of LF noise events showing a coherent shape (since incoherent noise would average to roughly 0 on the y-axis) on T2Z1 (left) T2Z2 (center) and T2Z3 (right). . . . 118

4.29 (left) A two-level decision tree. (right) A diagnostic plots of the error rate, for both training and test data, to help select the optimal number of weak decision trees. . . . . . . . 120

4.30 (top) The feature discrimination power of different BDT parameters, which is calculated using the amount that each feature split point improves the sample purity, weighted by the number of data points in the branch of interest, and summed over many decision branches. (bottom) The Run 3b BDT score as a function of energy, showing good separation of a small collection of signal events from the population of noise events . . . . . . . . . . . . . . . . . . 122

4.31 The CDMSlite R3 low background WIMP-search spectrum with a relatively loose cut placed on the LF noise BDT score. We seek to answer how many of the low energy events are LF noise triggers as a function of the cut value, and then set a cut such that there is $<1$ event leakage. . . . . . . . . . . 123

4.32 The 4 boxes into which events are divided when two bifurcated cuts (cut A and cut B) are applied to the data. The number of events in the upper left box $\left(N_{A B}\right)$ represents the number of events that pass both cuts. . . . . . . . . . . 124

4.33 (top) The two branches of the bifurcated BDTs of Run 3a data, colored by the full BDT score. (middle) The setup cut value in the full BDT score. (bottom) The distribution of events considered for the bifurcated analysis shown in black, with those rejected by the setup cut shown in blue. . . . . . 128

4.34 The distribution of events failing the setup cut, characterized with a kernel density. Integrating the kernel density into the signal region gives 0 events. . . . . . . . . . . . . 129 
4.35 (left) A toy model of a background distribution (blue) and a signal distribution (red) with known correlation coefficient in the two variables. (right) The full signal + background distribution, where the correlation of the background distribution is estimated using the method described above, and agrees with the true correlation coefficient. . . . . . . . . . . 130

4.36 (left) The distribution of simulated signal in the bifurcated analysis cut plane. $97.5 \%$ of the simulated signal is contained in the passage box, while $0.47 \%$ is in the $A \bar{B}$ box, $1.9 \%$ is in the $\bar{A} B$ box, and $0.13 \%$ is in the $\bar{A} \bar{B}$ box. (right) The distribution of the DM search data in the bifurcated analysis cut plane. The points in the shaded pink area pass both bifurcated cuts. . . . . . . . . . . . . . 131

4.37 Examples of cut loosening when the cuts are uncorrelated (left) and correlated (right). . . . . . . . . . . . 131

4.38 (top) The number of events in the $A B$ box, as the value of cut $\mathrm{B}$ is loosened. (middle) The estimated number of signal events outside the $A B$ box, as the value of cut $\mathrm{B}$ is loosened. (bottom) The number of new events in the $A B$ box minus the number of estimated signal events shown in red, as well as the bifurcated analysis's leakage estimate in blue. . . . . . 133 
4.39 (top left) Calculated voltage map for high radius events, showing the difference in electric potential $\Delta V$ between the final collection points of the positive and negative charge carriers, as a function of initial position of the pair (plotted as radius squared vs. vertical position). Here, the top of the crystal is biased at $75 \mathrm{~V}$ and the bottom is grounded. Charge carriers in the outermost (radius $>800 \mathrm{~mm}^{2}$ ) detector annulus can experience less than the full detector bias voltage (credit: D. Barker [113]). (top right) A cartoon of the radial vs. energy distribution for a homogenous and monoenergetic background in the CDMSlite detectors. The $P_{i}$ and $P_{O}$ refer to inner and outer probabilities, and these variable names are used in the radial cut efficiency calculation of Sec. 4.9 .5 (credit: M. Pepin [82]). (bottom left) Distribution of the radial parameter $\xi$ vs. energy in the DM search data. An energy-dependent cut on $\xi$ defines the fiducial volume below $2 \mathrm{keV}_{\text {ee }}$, while a stricter constant cut is used above $2 \mathrm{keV}_{\text {ee }}$. (bottom right) Resolution $(1 \sigma)$ for $\xi$ (radial parameter) shown as a function of $\xi$ and energy. At lower energy, the resolution worsens as the increased noise affects the reconstruction of the radial parameter. (credit: R. Underwood [114]) . . . . . . . . . . . 134

4.40 The Monte Carlo distribution of reduced NTL events in the $2 \mathrm{~T}$ radius (i.e. $\xi$ ) vs. energy plane. The colored points are those that would pass a cut such that (after normalizing the Monte Carlo to the Run 3 exposure) only 1/8th of an event would pass into the signal region. (credit: R. Underwood) . 138

4.41 Livetime contribution of each series block to the R3 exposure, with the livetime removed by the cuts listed in Sec. 4.9.1 shown at the top of each bar of live days. . . . . . . . . . 139

4.42 (top) Trigger calculation showing the number of events that triggered both T2Z1 and the other detectors (numerator counts) as well as the number of events that triggered just the other detectors (denominator counts). (bottom) Binned efficiency of the trigger as a function of energy. Error bars are binomial statistical uncertainties. . . . . . . . . . . . . . . . 141

4.43 (left) Corner plot for trigger efficiency fit of $\mu$ and $\sigma$. (right) Best-fit error function with $100 \mathrm{MC}$ sample curves from the $\mu$ vs. $\sigma$ posterior. These samples serve as visualizations of the model uncertainties. . . . . . . . . . . . . . . . . 142 
4.44 A cartoon depicting the pulse simulation procedure (credit A. Anderson: [120]). . . . . . . . . . . . . . . 143

4.45 (left)The fast/slow amplitude ratio, as a function of energy, for the two-template fit optimal filter fit. (center) (right) Two $L$-shell pulses showing the variation in pulse peakiness where the center pulse has is the peakiest of the $L$-shell events (fast/slow ratio $=0.4$ ) and the right pulse is the least peaky of the $L$-shell events (fast/slow ratio= -0.6). . . . . . . . . . 144

4.46 (left) The distribution of simulated signal events in the $\Delta \chi_{\text {glitch }}^{2}$ vs energy plane with the cut boundary overlaid. (center) The efficiency of the $\Delta \chi_{\text {glitch }}^{2}$ cut calculated with data the simulated signal data. (right) The efficiency of all DQ cuts calculated using Eq. 4.26. All figures use series block 7 simulated data and cut boundaries. . . . . . . . . . . . . . 145

4.47 The signal efficiency with successive application of the trigger efficiency, quality cuts efficiency, and fiducial volume cut efficiency. The final data is included with statistical and systematic $1 \sigma$ uncertainty. Fitting the efficiency model to these data gives the final (blue) efficiency curve and the corresponding $\pm 1 \sigma$ uncertainty band. . . . . . . . . . . 148

5.1 Fits of a Gaussian + linear background to the energy spectra of zero-energy (baseline) events and events from each ${ }^{71} \mathrm{Ge}$ activation peak. The widths of the Gaussians are the energy resolution $\sigma$. Copied from [126] with permission. (credit: D. Barker) . . . . . . . . . . . . . . . 153

5.2 The spectra (normalized to event density) of surface events expected from the three surface background locations (left: germanium; center: housing; right: top lid). For each location, the solid curve represents the mean of the expected event distribution $\left(\rho_{0}\right)$. The shaded band shows the $1 \sigma$ uncertainty, where the top and bottom edges of the bands correspond to $\rho_{+}$and $\rho_{-}$in Eq. 5.7, respectively. Copied from [126] with permission. (credit: D. Barker) . . . . . . . . . . . 158

5.3 (left) The distribution expected (with detector efficiency applied) from a $7 \mathrm{GeV} / c^{2} \mathrm{DM}$ particle for different values of the Lindhard $k$ parameter. (right) Background distributions at the magnitude expected in the Run 3 data (estimated prior to unblinding). . . . . . . . . . . . . 160 
5.4 (left) Uncertainty on the shape of the housing background. (right) The uncertainty on the shape and normalization of the housing background, as controlled by a single morphing parameter that is given by a single color. . . . . . . . . . . 161

5.5 (left) Likelihood fit, to a pseudo-dataset, with WIMP cross section freely floating. (right) A likelihood fit, to the same pseudo-dataset, with WIMP cross section clamped to $\sigma_{\chi}=$ $2.3 \times 10^{-42} \mathrm{~cm}^{2}$. On both plots, the best fit number of background events from the different backgrounds are shown in the legend, and the likelihood values are shown in the plot title. . . . . . . . . . . . . . 166

5.6 An example $q_{\sigma_{\chi}, \text { obs }}$ relative to a MC produced $f\left(q_{\sigma_{\chi}} \mid \sigma_{\chi}\right)$ distribution, as well as the theoretical $(1 / 2) \times\left(\delta(0)+\chi^{2}\right)$ distribution. . . . . . . . . . . . . . 167

5.7 Cramér-von Mises Statistic of the fit to the data (red line) and 1000 pseudo experiments. . . . . . . . . . . 170

5.8 (left) The R3 final spectrum with all cuts applied, and with salt included and highlighted). (right) The distribution of salt (with no cuts applied) highlighted in the energy vs. $\xi$ radial parameter plane, with the real data shown in the background. 171

5.9 (left) The salt passage fraction with sequential application of cuts (shown in the legend) compared to the signal efficiency estimate. (center) The energy spectrum of events, in the unsalted dataset, selected to be replaced by salt events. Roughly $19 \%$ of them were multiples, consistent with the passage fraction given by the left hand figure. (right) The salt passage fraction, but without the multiples and muon veto cut. . . . 172

5.10 (top) Background best fit amplitudes overlaid on the Run 3 unsalted data. "Sf" is an abbreviation for "surface". (bottom) 1D and 2D posterior distributions for the number of events (or morphing parameter in the case of surface backgrounds) contributing from each background. By posterior, we do not mean to imply that these are the results of a Bayesian analysis; rather, these are the fit results from the likelihood, with asymmetric uncertainties determined with a Markov-chain Monte Carlo sampling algorithm. . . . . . . 173 
5.11 Comparing prior constraints with fit results of the maximum likelihood for the efficiency, morphing, and resolution parameters. The similar variance of the prior and posteriors indicates that the data are not providing additional information for these systematic uncertainty parameters, and the similar mean indicates that our signal efficiency and resolution model parameters are in agreement with the data. . . . . . . . . 174

5.12 (left) R3 $1 \sigma$ sensitivity band and PLR limit (with R2 OI limit overlaid) . . . . . . . . . . . . . 177

5.13 The CDMSlite Run 3 90\% CL PLR limit (this result, solid black) on the spin-independent WIMP-nucleon cross section, along with the $\pm 1 \sigma$ and $\pm 2 \sigma$ sensitivity bands (green and yellow respectively). The CDMSlite Run 3 optimum interval limit (dashed grey) and Run 2 (red) optimum interval limit [143] are overlaid. Examples of limits from other detector technologies are overlaid: DarkSide-50 2018 No Quenching Fluctuations (magenta) [84]; PandaX-II 2016 (blue) [43]; PICO-60 2017 (orange) [144]; CRESST-II 2016 (cyan) [94]; CDEX-10 2018 (purple) [145] . . . . . . . . . . . . . . 177

6.1 The dashed lines show the projected 90\% exclusion sensitivity of the SuperSCDMS SNOLAB experiment, calculated using the optimum interval method. The y axis is the spinindependent WIMP-nucleon cross section. The solid lines show the current 90\% from the CRESST-II [94], SuperCDMS [126, 143], and LUX [147] experiments. The dotted yellow line is the region of parameter space where the solar neutrino coherent elastic scatter rate begins to mask the interaction rate from DM particles, as calculated in Ref. [148]. Figure from SuperCDMS approved public plots. . . . . . . . . . 180

A.1 (left) The zoomed-in version of the left hand plot highlights the discrepancy between the expected, by extrapolation, location of the $K$-shell peak at $0 \mathrm{~V}$. (right) The location of the $K$ - and $L$ - shell peaks as a function of detector voltage. The higher voltage peaks $(30 \mathrm{~V}, 50 \mathrm{~V}$, and $70 \mathrm{~V})$ are linear to within uncertainty, but there is a deviation from linearity at $0 \mathrm{~V}$. The overall energy scale (y axis) has used a calibration constant for the detector obtained at $4 \mathrm{~V}$, though the overall scaling of the $y$ axis is irrelevant when checking for linearity. 
A.2 Large systematics in Luke phonon collection (15\%) and recombination phonon collection (50\%) would contribute at most to a $\sim 3 \%$ error in the nuclear recoil energy scale. The recombination phonon error becomes negligible at high detector bias. Also the error decreases at higher detector voltage, $V_{b}$, as the total phonon energy becomes more correlated with the ionization. . . . . . . . . . . . . . . 213 


\section{Glossary}

$\xi$ Two Template Radial Parameter

$\mathbf{2 T}$ Two Template, in relation to the optimal filter fitting algorithm

BDT Boosted Decision Tree

CL Confidence Level

CMB Cosmic Microwave Background

$\mathbf{C L}_{\mathrm{S}}$ Limit setting method that protects against downward fluctuating background rates

CryoPreTime the cryocooler vibrational phase (see crycooler)

CVM Cramér-von Mises

cryocooler The most vibrationally intense component of the SuperCDMS Soudan cryogenic equipment that generates noise in the detectors

DAQ Data Acquisition (System)

DFT Discrete Fourier Transform

DOF Degree of Freedom

DM Dark Matter

EDELWEISS Expérience pour DEtecter Les WIMPs En Site Souterrain (Experiment to Detect WIMPs in Underground Site) 
EC Electron Capture

ER Electron Recoil

FET Field Effect Transistor

G23R A germanium prototype detector, with the same dimensions and channel layout as the SuperCDMS Soudan iZIP detector, though only instrumented on one side

GOF Goodness of Fit

HS Housing (for surface backgrounds)

HV High Voltage

iZIP interleaved Z-sensitive Ionization and Phonon Detector

KDE Kernel Density Estimate

$\mathbf{k e V}_{\text {ee }} \mathrm{keV}$ energy of an event, converting the energy to that produced by an electron recoil (ee: "electron equivalent")

$\mathbf{k e V}_{\mathrm{nr}} \mathrm{keV}$ energy of an event, converting the energy to that produced by a nuclear recoil (nr: "nuclear recoil")

$\mathbf{k e V}_{\mathrm{t}} \mathrm{keV}$ energy of the total phonon energy of an event

LF Low Frequency

LFN Low Frequency Noise

LT Live Time

MC Monte Carlo

NR Nuclear Recoil

NTL phonons Neganov-Trofimov-Luke phonons 
OF Optimal Filter

OI Optimum Interval method

PDF Probability Density Function

PLR Profile Likelihood Ratio

PSD Power Spectral Density

pt2T Total phonon energy, estimated with the two template optimal filter algorithm, in units of $\mathrm{keV}$

ptOF Total phonon energy, estimated with the 1D optimal filter algorithm, in units of $\mathrm{keV}$

ptOF0 Total phonon energy, estimated with the 1D optimal filter algorithm with the time delay forced to 0 , in units of $\mathrm{keV}$

ptNF Total phonon energy, estimated with the non-stationary optimal filter algorithm, in units of $\mathrm{keV}$

R2 CDMSlite Run 2, which occurred over the course of 2014 and consisted of operating the T5Z2 detector at $70 \mathrm{~V}$

R3 CDMSlite Run 3, which occurred over the course of 2015 and consisted of operating the T2Z1 detector up to $75 \mathrm{~V}$

R3a The first portion of CDMSlite Run 3, before the voltage was stabilized and in which the detector noise performance was better

R3b The second portion of CDMSlite Run 3, after the voltage was stabilized and in which the detector noise performance was worse

Random Trigger A time series of data that is triggered randomly with the intention of reading out a time series in which no event occurs in order to characterize detector noise. Also referred to as just a "random."

RMS Root Mean Squared 
ROI Region Of Interest

S12C A Silicon prototype detector, with the same dimensions and channel layout as the SuperCDMS Soudan iZIP detector

SHM Standard Halo Model

SN Series Number

SNOLAB Sudbury Neutrino Observatory Laboratory

SUSY Supersymmetry

SQUID Superconducting Quantum Interference Device

$\boldsymbol{T}_{c}$ Superconductor critical temperature

T2Z1 An iZIP detector used in the SuperCDMS Soudan experiment, and in CDMSlite Run 3, in Tower 2 and the first (highest) position

T5Z2 An iZIP detector used in the SuperCDMS Soudan experiment, and used in CDMSlite Run 1 and Run 2, in Tower 5 and the second (middle) position

TES Transition Edge Sensor

TL Top Lid (for surface backgrounds)

WIMP Weakly Interacting Massive Particle

z4z5 correlation Waveform correlation between the Z4 (T2Z1) detector and Z5 (T2Z2) detector

z4z6 correlation Waveform correlation between the Z4 (T2Z1) detector and Z6 (T2Z3) detector 


\section{Acknowledgements}

First I would like to thank Scott Oser for his supervision over the past five years. Scott was always available when I needed it and the quality of the guidance he offered - from detailed analysis help to general organizational advice - will always amaze me. Scott gave me opportunities to broaden my research experience by allowing me to work on a range of topics related to CDMS, and this made a real difference in my appreciation of such a complex experiment. One thing that struck me midway through this degree is that I always left meetings with Scott feeling more focused, and feeling better about life in general, and I take this to be one mark of a great advisor.

Within the CDMS collaboration I have worked with many kind and intelligent people. Over the past year I worked most closely with D'Ann Barker as we inched the CDMSlite Run 3 analysis towards completion with weekly group meetings, Skype calls, and countless emails. I was very lucky to have D'Ann as a partner as we pushed through the challenges of coordinating a large group effort within a collaboration like SuperCDMS. I can only hope that my future work partners will be as knowledgeable and on top of things as her. Mark Pepin was particularly generous with his time whenever I had a question related to CDMSlite, and in multiple ways he paved the way for the CDMSlite Run 3 analysis. Wolfgang Rau provided valuable advice on many topics in this thesis, and we were lucky that one of the foremost detector experts connected so frequently to our analysis calls to share his knowledge. Rob Calkins was a great help in coordinating analysis work. Bruno Serfass was an approachable source of knowledge on anything CDMS related even when I was new to the experiment and more of a time sink than a source. Matt Pyle was also exceptionally generous with his time to teach me about detector physics while I was visiting Berkeley. His enthusiasm for his research is something to aspire to. It has also been a pleasure to work alongside and/or learn from Nick Zobrist, Arran Phipps, Noah Kurinksy, Ben Loer, Lauren Hsu, Jorge Morales, Steve Yellin, Tsuguo Aramaki, Ryan Underwood, Nick Mast, Anthony Villano, Dan Jardin, and Matt Fritts.

At UBC, I thoroughly enjoyed the two years of Danika MacDonell's company in the office, and I will miss summer volleyball at Jericho beach

xxxiv 
with her. Working with Belina von Krosigk was also a pleasure. To Alon Hershenhorn, Emanuele Michielin, and the numerous office mates who have passed through room 222, thank you for your willingness to put down your work for me to bounce my own ideas off of you. I also must state my gratitude to the UBC physics department as a whole. It has been a stimulating, and at times quite humbling, experience to be around so many impressive scientists over the past five years.

Last, thank you also to all my sailing, scrambling, climbing, and ski buddies who accompanied me on adventures in Vancouver and the surrounding wilderness. 


\section{Chapter 1}

\section{Detection of Particle Dark Matter}

\subsection{Early Evidence}

In 1933 Fritz Zwicky calculated the mass of the Coma galaxy cluster by way of the virial theorem, using the velocity dispersion of galaxies from the measurements taken by Hubble and Humason [1], and found a mass-toluminosity ratio of roughly 500, significantly higher than expected for galaxy clusters [2]. Three years later Sinclair Smith performed a similar analysis of the Virgo galaxy cluster and observed a significant discrepancy, by a factor of about 200, between the expected mass per galaxy from luminosity measurements and his calculation of the mass per galaxy [3]. The term "dark matter" was being used in the literature at this point, but it was not thought of in today's paradigm, with Smith asserting that the discrepancy was due to uniformly distributed or low luminosity internebular material [4].

After Zwicky and Smith, more accurate measurements were made of the mass of galaxies, many of which were based on the rotational speeds of nearby galaxies. At high galactic radii within which the majority of luminous matter is enclosed, if all the mass in the galaxy were luminous then Newton's and Gauss' laws predict the rotational speed of orbital bodies around the galactic center to be:

$$
v(r)=\sqrt{G M_{l}} \sqrt{\frac{1}{r}}
$$

where $M_{l}$ is the mass of the luminous matter. Horace Babcock in 1939 measured rotational speeds of the Andromeda galaxy (M31) and found rising speeds out to distances beyond where the luminous matter was enclosed (100 arc minutes), in tension with Eq. 1.1. He attributed the measurements to large amounts of non-luminous matter in the outer part of the galaxy or to new dynamics that would account for the high rotational speeds without a new mass component $[4,6]$.

We now know definitively that the rotation speed of objects around the 


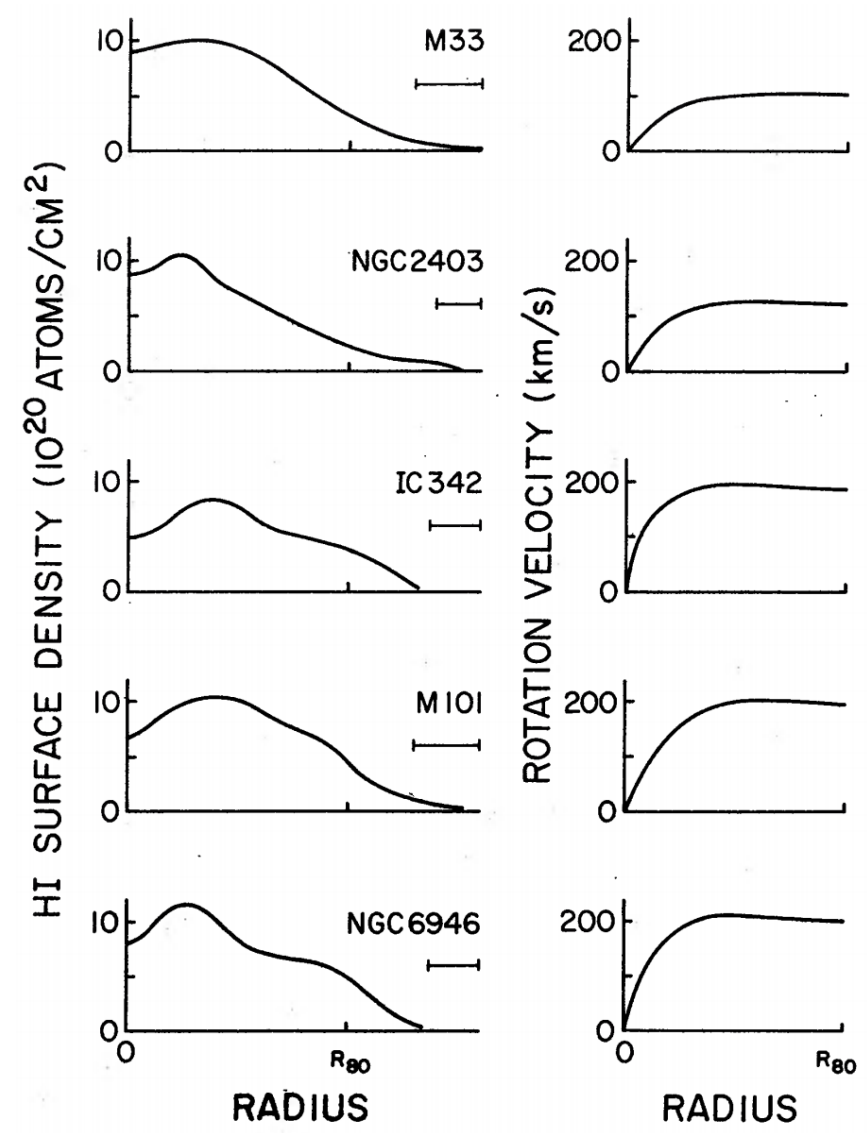

Figure 1.1: (left) The hydrogen surface densities (azimuthally averaged) for five galaxies and (right) the rotation galactic rotation curves. The bars under the galaxy names indicate the spatial resolution of the measurements. $\mathrm{R}_{80}$ corresponds to the galactic radius containing $80 \%$ of the Hydrogen density determined from the $21 \mathrm{~cm}$ emission. Plot copied from Ref. [5], with permission. Measurements were performed by Rogstad and Shostak in 1972 from the Owens Valley Radio Observatory using $21 \mathrm{~cm}$ observations. 


\subsection{Galaxy Clusters}

galaxy does not fall off as $r^{-1 / 2}$. In the 1960s and 70s, using the Doppler shift of the $\mathrm{H} \alpha$ (optical) hydrogen emission line, Vera Rubin and Kent Ford measured the rotation speeds of hydrogen in M31, showing that the speeds are roughly constant at large radius from the galactic center [7]. Subsequent measurements of a host of spiral galaxies by Rubin, Ford, and Thonnard also showed flat rotation curves [8]. Outside of the optical band, Rogstad and Shostak analyzed the rotation curves from five galaxies using hydrogen's $21 \mathrm{~cm}$ photon emission [5]. The flat rotation curves measured for these five galaxies are shown in Fig. 1.1.

To explain the flat rotation curves, if we instead modeled mass in the galaxy as a spherically symmetric distribution that varies with distance, $M(r)$, the constant stellar rotational velocity at large distances given by

$v=C=\sqrt{G M(r)} \sqrt{\frac{1}{r}}$, can be explained by an enclosed mass that varies linearly with the distance from the center:

$$
M(r) \propto r .
$$

Therefore the enclosed mass has a density proportional to the inverse square of the distance from the galactic center, $\rho(r) \propto \frac{1}{r^{2}}$. Most of this matter must be dark because the luminous mass density falls off much more rapidly. To account for this missing matter, the leading model is a spherically symmetric "halo" of dark matter distributed throughout our galaxy and other galaxies, interacting gravitationally and making up the majority of mass in the galaxy and the universe.

\subsection{Galaxy Clusters}

Measurements of galaxy clusters since the work of Zwicky and Smith have provided additional evidence for dark matter and important information regarding the composition of dark matter. Observations of galaxy clustersthe largest gravitationally collapsed astrophysical structures - are particularly powerful because different techniques can be used to make independent measurements of their mass.

The Chandra Observatory measures the x-ray emission from the intergalactic gas of galaxy clusters. With the clusters' gravitational potential largely due to dark matter, their intergalactic gas gains kinetic energy and heats to $\sim 10^{8} \mathrm{~K}$, and they are therefore among the brightest x-ray sources. By measuring the x-rays, the temperature and pressure of the gas can be computed. If the cluster is in equilibrium, the gravitational potential can be computed under the good assumption that gravitational forces cancel with 


\subsection{Galaxy Clusters}

pressure forces. Recent publications matching models of the interstellar gas and dark matter halo to Chandra Observatory data indicate that ordinary matter makes up 12-15\% of the total mass of the cluster [9].

In an independent measurement of the cluster mass, the Hubble Space Telescope measures light from background objects that bends around the massive structures. The total gravitational mass of the cluster is computed by the strength of the gravitational lensing, which confirms that the majority of gravitational mass of the clusters is not due to the luminous matter[10].

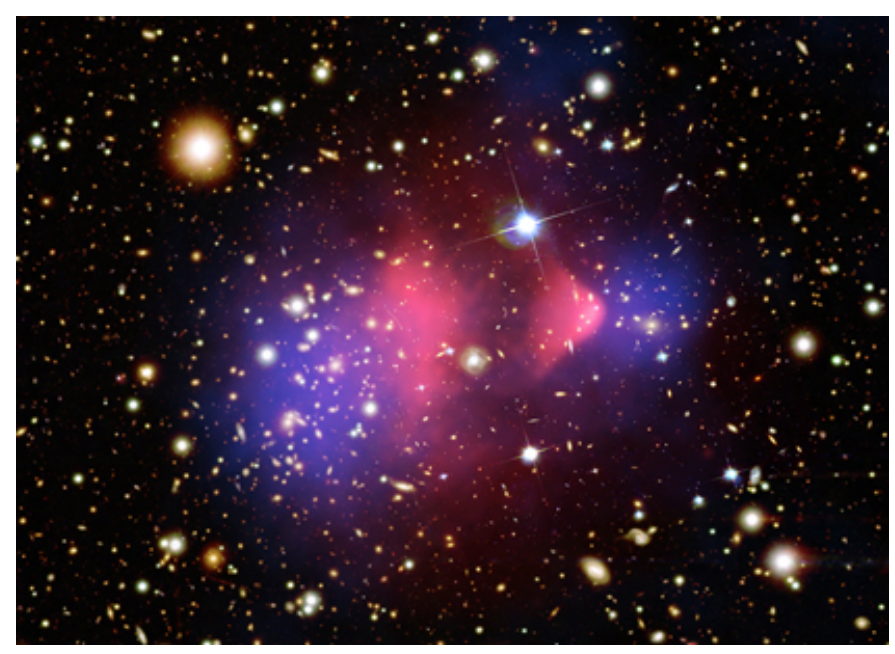

Figure 1.2: 1E 0657-56 (C) 2004 X-ray: NASA/CXC/CfA/M.Markevitch et al.; Optical: NASA/STScI; Magellan/ U.Arizona/ D.Clowe et al.; Lensing Map: NASA/STScI; ESO WFI; Magellan/ U.Arizona/ D.Clowe et al., by permission) [11] [12] .

The "Bullet" Cluster is one of the most famous examples of the dark matter making itself apparent in our universe. The Hubble Space Telescope and Chandra Observatory have observed the collision of two galaxy clusters and measured the distributions of both the gravitational matter measured with strong gravitational lensing and luminous matter measured with x-ray emission. As shown in Fig. 1.2, these two distributions are observed to have separated. The luminous matter (pink) is superimposed on the distribution of gravitational matter (i.e. predominantly dark matter) as measured by lensing (purple). The two dark matter distributions have passed through each other while the luminous distributions lag behind due to the impedance of their collisions. The nearly non-interacting dark matter streams through unimpeded. 


\subsection{Big Bang Nucleosynthesis}

Measurement of the abundances of light elements produced in the early universe was an important step in constraining the dark matter composition hypotheses. These measurements indicated, using a different set of observables (and different systematics) than the measurements discussed in Sec. 1.1 and 1.2, that the baryon density of the universe was significantly smaller than the total matter component. These measurements not only pointed to the existence of dark matter, but also indicated that dark matter must be non-baryonic.

In the first minutes after the Big Bang the energy of photons dropped below the binding energy of deuterium $(2.2 \mathrm{MeV})$, allowing protons and neutrons to fuse into deuterium $\left(\mathrm{p}+\mathrm{n} \rightarrow{ }^{2} \mathrm{H}+\gamma\right)$. Because both ${ }^{3} \mathrm{He}$ and ${ }^{4} \mathrm{He}$ have higher binding energies than deuterium, subsequent reactions produced those nuclei. The binding energies of nuclei in the $A=5-8$ range are lower than that of ${ }^{4} \mathrm{He}$, and so combined with the fact that particle densities at this stage of the universe forbade 3-body interactions, light nuclei production stopped (to first order) at ${ }^{4} \mathrm{He}$. Therefore, only ${ }^{2} \mathrm{H},{ }^{3} \mathrm{He}$, and ${ }^{4} \mathrm{He}$ were produced in significant quantities in the early universe (with small amounts of ${ }^{7} \mathrm{Li}$ ). The abundances of these nuclei are sensitive to the baryon density in the early universe. For example, the efficiency of the primary reaction that turns deuterium into helium, ${ }^{2} \mathrm{H}+\mathrm{p} \rightarrow{ }^{3} \mathrm{He}+\gamma$, depends on the proton number density. Models of these nuclear processes predict abundances shown as colored bands in Fig. 1.3, and show a strong dependence on the baryon density.

The measurements of these abundances in today's universe are complicated by the fact that the high densities within stars do result in 3-body interactions, allowing for nuclei production heavier than ${ }^{4} \mathrm{He}$. Measurements of the deuterium abundance, made by observing high redshift quasars and exploiting the difference in the absorption properties of deuterium to hydrogen, provide the best estimates for the baryon density [13]. This measurement is shown by the smallest yellow box in Fig. 1.3. The baryon to photon ratio of $\sim 6 \times 10^{-10}$ translates to a $\sim 5 \%$ baryon mass-energy density of the universe, which is significantly smaller than the mass density needed to explain galactic rotation curves and to explain measurements of the cosmic microwave background, discussed next in Sec. 1.4. 


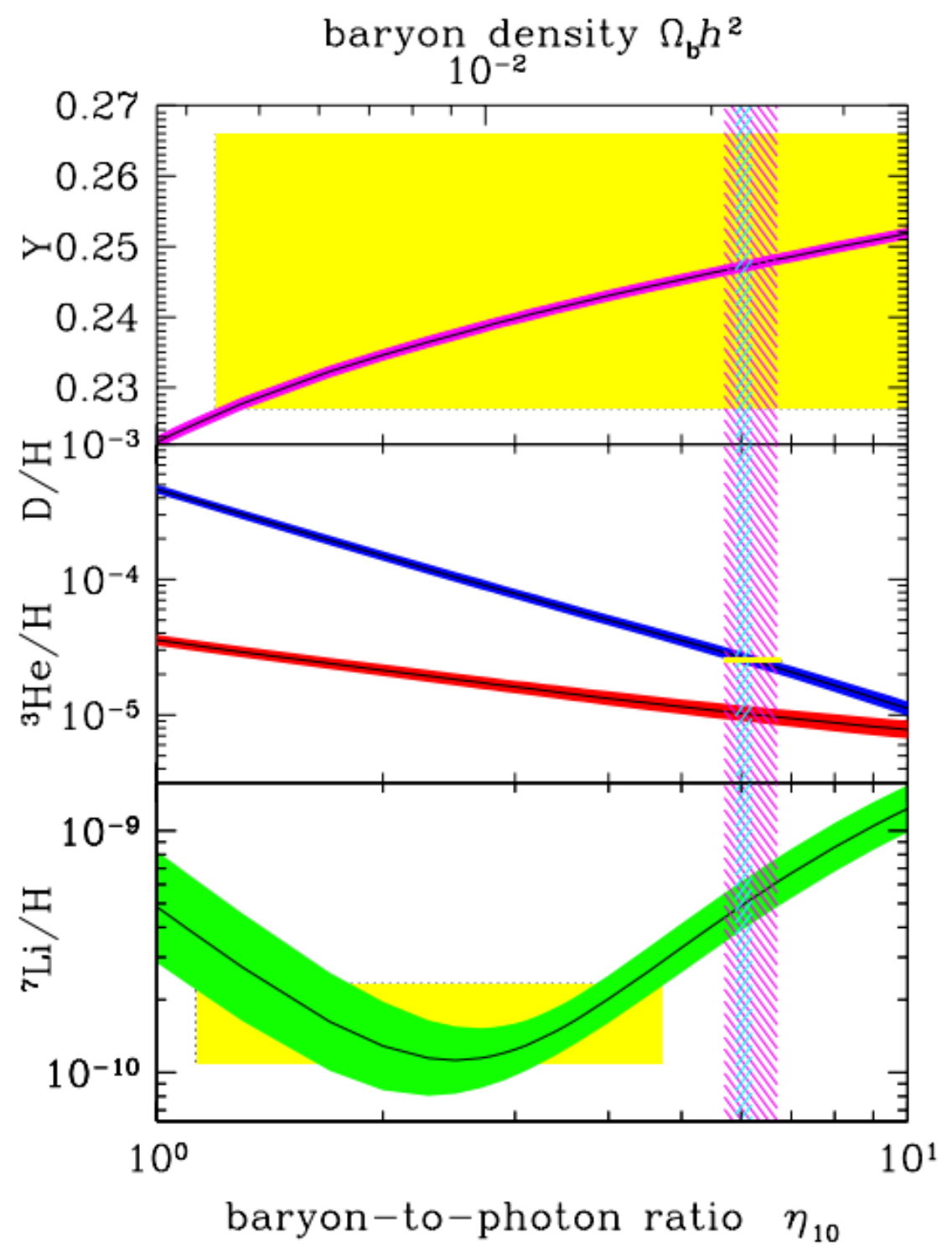

Figure 1.3: $Y$ is the ${ }^{4} \mathrm{He}$ mass fraction of all baryons. The blue, red, and green bands are the modeled number densities of ${ }^{2} \mathrm{H},{ }^{3} \mathrm{He}$, and ${ }^{7} \mathrm{Li}$, respectively, relative to ${ }^{1} \mathrm{H}$ as a function the baryon density. Yellow boxes show measurements (95\% CL) of the light element abundances; of note is that only upper limits on ${ }^{3} \mathrm{He}$ abundance have been made, and the measurements of ${ }^{7} \mathrm{Li}$ are in slight tension with the model. The pink hatched band shows the inferred baryon to photon ration from the ${ }^{2} \mathrm{H}$ measurement, while the blue hatched band shows the range indicated by CMB measurements. From [14] with permission. 


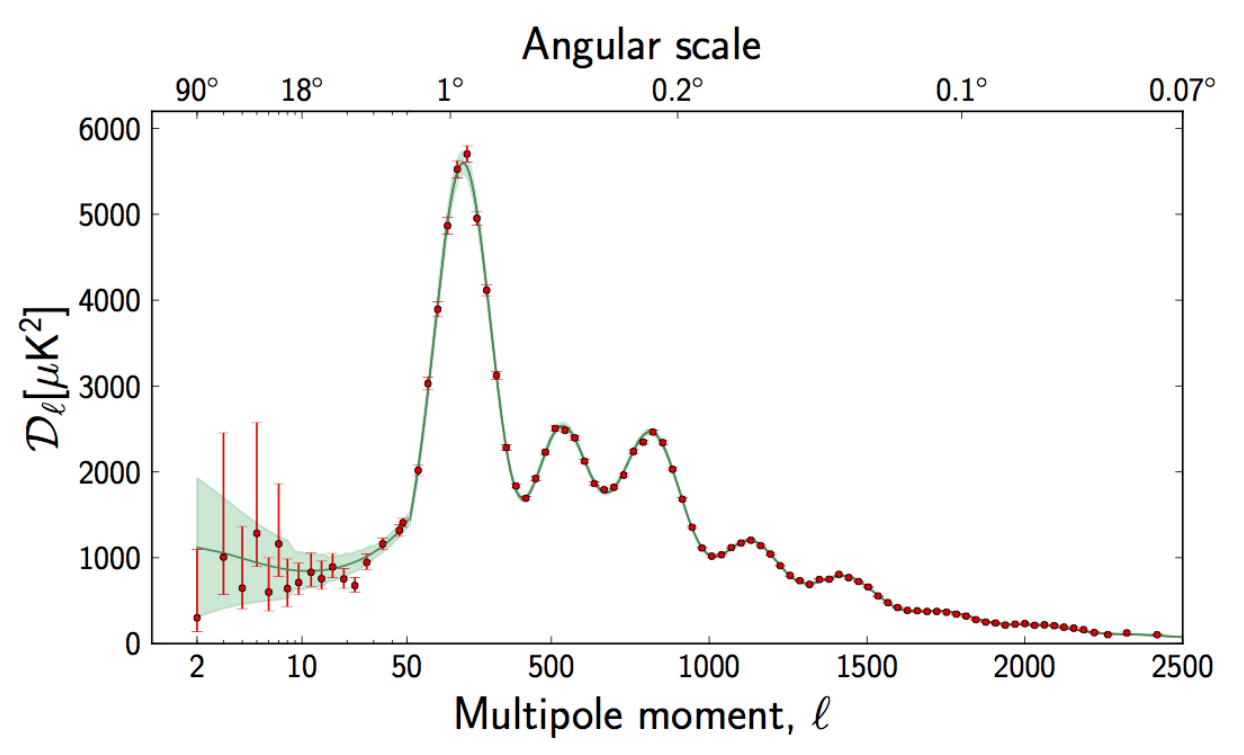

Figure 1.4: CMB power spectrum predicted by a $\Lambda$-CDM cosmology (i.e. a dark energy (69\%) and cold dark matter (26\%) dominated universe). Data points in red are measurements by the Planck collaboration [15]. Power spectrum of temperature fluctuations in the Cosmic Microwave Background (c) 2013 ESA/Planck, and the Planck collaboration, from Planck 2013 results. I. Overview of products and results, by permission).

\subsection{The Cosmic Microwave Background}

The Cosmic Microwave Background (CMB) provides the most accurate measurement of the fraction of the dark matter density of the universe. Approximately 400,000 years after the Big Bang, the expanding universe cooled to a point where it became energetically favorable for the plasma of protons and electrons to fall out of equilibrium with photons and form neutral hydrogen. At this point of "recombination," the universe became transparent to the photons which make up the CMB radiation, which matches a blackbody spectrum with (currently) a temperature of $2.7 \mathrm{~K}$. Slight differences, or anisotropies, in this temperature across the sky, on the order of $100 \mu \mathrm{K}$, have been a rich source of cosmological information, including the most accurate measure of the non-baryonic (dark matter) matter density in the universe.

Transforming the spatial anisotropies into spherical harmonics gives the CMB power spectrum, or the variance of the spatial fluctuations as a function of angular scale. The acoustic peaks of the power spectrum show the 
angular scales at which the photons were slightly hotter and slightly colder than average at recombination. The hotter overdensities are regions where the photons, coupled to the baryonic matter up to the point of recombination, had clumped together because of gravitational wells into which the baryonic matter was attracted.

Importantly for the case of the non-baryonic dark matter, the amplitude of the peaks depends on the dark matter density in the universe. This is because the pressure of the photons coupled to the baryonic matter opposes the formation of gravitational wells. In order to accurately model the location and amplitude of the peaks, a decoupled non-baryonic matter component must exist which continues to collapse regardless of the photon restoring force. The photon pressure restoring force does set up an oscillation of the baryon-photon plasma, which is highly sensitive to the non-baryonic matter density, and which is imprinted on the CMB at last scattering in the form of the peaks in the power spectrum [16]. With the CMB power spectrum we are therefore able to determine the non-baryonic matter density. The CMB power spectrum is sensitive to numerous other properties of the universe, such as the dark energy fraction, and these cosmological parameters are fit in a multidimensional space to the power spectrum. The best current measurement of the cold dark matter density fraction of the universe is $26.8 \%$ (with a $68.3 \%$ dark energy component) [17]. Reference [17] contains further details on the power spectrum fitting, including discussion of the covariances between cosmological parameters.

\subsection{Composition Hypotheses}

Despite overwhelming observational evidence that dark matter does exist, very little is known about its composition. A number of theories have been put forth.

In one effort to account for the dark matter, experiments searched for hidden massive compact halo objects (MACHOs), such as black holes or massive non-luminous planets. They looked for MACHOs in the Milky Way by waiting for slight unexpected gravitational lensing of distant luminous galaxies as a MACHO passed between us and the galaxy. These searches ruled out the possibility of MACHOs constituting any more than $25 \%$ of the Milky Way's dark matter halo, and therefore disqualified them as the primary dark matter candidate [18].

Most theories predict that non-baryonic particles make up dark matter halos around galaxies, but still there exist many possibilities for the type of 
particle. If we assume that dark matter is non-baryonic, it is highly likely that such dark matter is also non-relativistic, or "cold", dark matter. Relativistic, or "hot", dark matter conflicts with the accepted model of galaxy formation [19]. Returning to the discussion of Sec. 1.4, had the dark matter been relativistic then its kinetic energy would have largely prevented its gravitational collapse. However, the gravitational landscape at recombination is well understood, and not only is it imprinted on the CMB but it also explains the formation of the dense small scale structures (galaxies) seen in the universe today. That the majority of dark matter is cold rules out a hot relativistic (neutrino-like) species from contributing substantially to the $26.8 \%$ dark matter component.

Theoretical models that predict new particles with these characteristics include, but are not limited to, axions [20, 21] and WIMPs [22]. In the following subsections we briefly review these and other candidates, with a focus on candidates to which CDMS detectors are sensitive.

\subsubsection{The WIMP Hypothesis}

The SuperCDMS experiment, along with many competitor experiments, searches for Weakly Interacting Massive Particles (WIMPs). The WIMP hypothesis is intriguing because it fits into a parameter space supported by supersymmetric (SUSY) theory as well as cosmology. A number of assumptions regarding matter in the early universe allow cosmologists to estimate the WIMP cross section. First, WIMPs would have been constantly created and annihilated until some critical point of the universe's cooling where the low temperature would prevent any further WIMP creation. Following this, expansion of the universe would have made it exponentially unlikely that a WIMP would collide with its antiparticle and annihilate [22]. This second critical moment - when annihilation ceases - is known as thermal relic "freeze out." The particle abundance resulting from freeze out depends on the WIMP annihilation cross section as shown in Fig. 1.5, with a detailed derivation given in Chapter 3 of Ref. [16]. In order to account for the dark matter in the universe, the WIMP annihilation cross section is estimated to be roughly at the scale of the weak force where yet undiscovered particles are expected to exist as postulated by SUSY [22].

This coincidence is what some refer to as the "WIMP miracle," since SUSY was initially proposed as a solution to other problems with the Standard Model of particle physics, but could naturally solve the dark matter problem as well. SUSY adds particles to the Standard Model and the lightest of these particles, the neutralino, could be the dark matter WIMP. This 


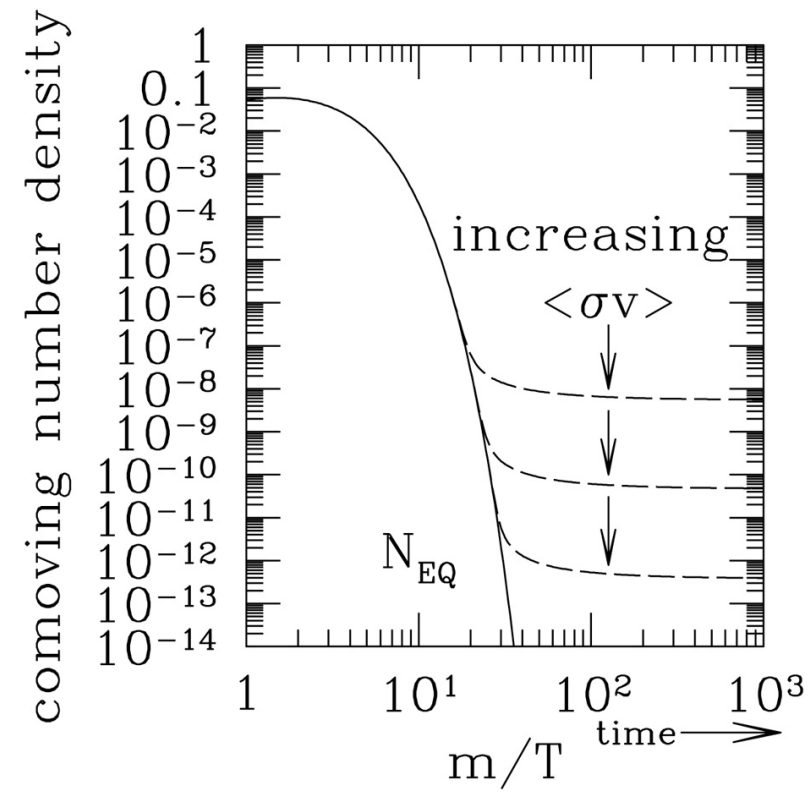

Figure 1.5: Number density of WIMPs in the Universe as a function of time, where the relic density depends on the WIMP annihilation cross section, $\sigma_{\chi \bar{\chi}}$ (C) NASA/IPAC Extragalactic Database (NED) which is operated by the Jet Propulsion Laboratory, California Institute of Technology, under contract with the National Aeronautics and Space Administration, by permission). 
convergence of SUSY and cosmology is the primary motivation behind the WIMP hypothesis and has launched the dozens of experiments attempting to detect WIMPs [22].

\subsubsection{Axions}

The axion was originally proposed as a solution to the strong charge parity (CP) problem $[20,21]$ and has since become a leading light mass $\left(10^{-5}\right.$ to $\left.10^{-2} \mathrm{eV}\right) \mathrm{DM}$ candidate. The strong CP problem refers to the observation that there is no violation of CP-symmetry in quantum chromodynamics, despite the fact that the theory contains $\mathrm{CP}$-violation. One manifestation of CP-violation would be an electric dipole moment in the neutron, but no such moment has been observed and upper limits [23] have been placed that are significantly lower than those naïvely expected from quantum chromodynamics. Peccei and Quinn [24] proposed a solution to the strong CP problem that includes a new particle (the axion) that cancels out the quantum chromodynamic effects that permit CP-violation.

Through interactions with standard model particles, axions would be produced in stars and provide an additional process for the stars to shed energy. Models of these processes combined with stellar lifetime values predict a $10^{-2} \mathrm{eV}$ upper limit on the axion mass [25]. The axion can convert to two photons, and terrestrial experiments search for this conversion in resonant cavities threaded by a strong magnetic field [26].

\subsubsection{Dark Sector Particles}

The DM could be a new particle from a "dark sector" that does not interact via the standard model force mediators. Dark sector theories in particular postulate the existence of particles in the lower mass range $\left(1-10 \mathrm{GeV} / c^{2}\right)$ [27-29], whereas the SUSY WIMP favors higher masses. One dark sector theory postulates a new force mediated by a dark photon, which can kinematically mix with the standard photon and therefore interact with standard model particles [27]. Interactions of such particles with protons can be searched for in detectors nominally designed for WIMP detection (which are discussed in Sec. 1.6.1).

A second class of dark sector models goes under the name of "asymmetric dark matter," and these models propose an asymmetry between the dark matter and its antiparticle [30-32], analogous to the baryon/anti-baryon asymmetry of the universe. Such models postulate the existence of DM particles in the $5-15 \mathrm{GeV} / c^{2}$ mass range [32]. Many asymmetric DM models 
exist, some of which fit into the SUSY framework, and some of which are motivated by new dark force mediators. It is not guaranteed that asymmetric DM interacts with standard model particles, but interactions are possible and could proceed via DM-proton scattering. In this case, again, detectors nominally designed for WIMP detection would be sensitive to these interactions [32].

\subsection{Dark Matter Detection}

\subsubsection{Direct Detection}

When DM WIMP particles interact in a detector on earth, the DM can lose energy by colliding with electrons and nuclei in the detector material. The amount of energy that the DM can lose is significantly greater for nuclei than electrons (as described in more detail below), and so direct detection experiments look for DM-nucleon recoils in terrestrial detectors and employ different targets (e.g. liquid argon, liquid xenon, germanium, silicon, calcium tungstate), background rejection techniques, amplifiers, and/or energy thresholds. Direct detection experiments are optimized to certain DM masses and cross sections based on their detector technology.

For a DM WIMP, the expected elastic scattering energy transfer to a target particle is given by:

$$
E_{\text {recoil }}=\left(\frac{m_{\chi} m_{T}}{m_{\chi}+m_{T}}\right)^{2} \frac{v^{2}}{m_{T}}\left(1-\cos \left(\theta_{\chi}\right)\right)
$$

where $m_{\chi}$ is the WIMP mass, $m_{T}$ is the target particle's mass, $v$ is the WIMP velocity, and $\theta_{\chi}$ is the WIMP scattering angle.

The WIMP velocity is given by $v$ and deserves brief discussion. In the Standard Halo Model (SHM) the DM halo is isothermal and isotropic. The phase space density for a DM particle in the halo, under these assumptions, is given by Maxwell-Boltzmann statistics

$$
f(\vec{x}, \vec{v})=C \exp \left(-E(\vec{x}, \vec{v}) / k_{B} T\right)
$$

with an energy given by

$$
E(\vec{x}, \vec{v})=\frac{1}{2} m_{\chi}|\vec{v}|^{2}+m_{\chi} \Phi(\vec{x})
$$

where $\Phi(\vec{x})$ is the gravitational potential and $C$ is a normalization constant. 
The velocity distribution function is calculated by integrating over the spatial coordinates

$$
\begin{aligned}
f(\vec{v}) & =\int d^{3} \vec{x} f(\vec{x}, \vec{v}) \\
& =C \exp \left(-m_{\chi} v^{2} / 2 k_{B} T\right) \int \exp \left(-m_{\chi} \Phi(\vec{x})\right) d^{3} \vec{x} \\
& =C^{\prime} \exp \left(-m_{\chi} v^{2} / 2 k_{B} T\right) \\
& =C^{\prime} \exp \left(-v^{2} / 2 \sigma_{v}^{2}\right)
\end{aligned}
$$

where in the last step we have defined the DM velocity dispersion as $\sigma_{v}^{2}=$ $k_{B} T / m_{\chi}$. The velocity dispersion is related to the characteristic velocity of the dark matter by $\sigma_{v}=\sqrt{1 / 2} v_{0}$. Since DM particles moving too fast will gravitationally escape the galaxy, $f(\vec{v})$ is generally truncated at the escape velocity ( $v_{\text {esc }}$ is taken to be $544 \mathrm{~km} / \mathrm{s}$ in the SHM, as estimated from high velocity stars [33]). Therefore, the final velocity distribution function is given by

$$
f(\vec{v})= \begin{cases}C^{\prime} e^{\left(-v^{2} / v_{0}^{2}\right)} & v<v_{e s c} \\ 0 & v>v_{e s c}\end{cases}
$$

The characteristic velocity of the DM is not a constant as a function of radial distance from the galactic center, and Ref. [34] shows that at radii comparable to the Sun's location, the characteristic velocity is approximately given by the local circular velocity at the radius of the Sun's orbit. Historically a value of $v_{0}=220 \mathrm{~km} / \mathrm{s}$ is used as estimated by galaxy surveys [35]. The earth is moving through this halo with a velocity $\vec{v}_{E}$, the vector sum of the sun's circular and peculiar velocity as well as a small (6\%) annual modulation due to the earth's orbital velocity around the sun. The SHM uses $v_{E}=232 \mathrm{~km} / \mathrm{s}$ for the average velocity of the earth, and boosts the DM velocity distribution into this frame with the transformation $\vec{v} \rightarrow \vec{v}+\vec{v}_{E}$ in Eq. 1.6.

One key element of direct detection of elastic scatters is made clear from Eq. 1.2 - the WIMP cannot efficiently transfer energy to target components that are much less massive than a nucleon. Consider the maximum energy transfer of a WIMP-electron collision $\left(\theta_{\chi} \rightarrow 180^{\circ}, m_{T} \approx m_{e}\right)$, giving $E_{\text {recoil }} \approx 2 m_{e} v^{2}=0.25 \mathrm{eV}$. Signals of this magnitude are "in the noise" projected for the even the lowest threshold next-generation dark matter experiments, although through upgrades detector resolutions for the following generation of experiments are projected to approach these values. 


\subsection{Dark Matter Detection}

Instead consider the maximum energy transfer of a WIMP-nucleon collision where the dark matter particle is well matched kinematically to a $\mathrm{Ge}$ nucleus target: $\left(\theta_{\chi} \rightarrow 180^{\circ}, m_{\chi} \approx m_{T} \approx 72 m_{p}\right)$. In this case $E_{\text {recoil }} \approx$ $(1 / 2) m_{T} v^{2} \approx 16.5 \mathrm{keV}$, which is certainly a detectable energy.

The local dark matter density is approximately $\rho_{\mathrm{dm}}=0.3 \mathrm{GeV} / \mathrm{cm}^{3}[36]$. Recent measurements of $\rho_{\mathrm{dm}}$ combine stellar kinematic data with simulation to estimate the local galactic gravitational potential in the vicinity of the Sun which is then converted to a dark matter density by subtracting out the contribution from luminous matter [37]. The measurements are prone to significant systematic uncertainties, though most measurements are within $2 \sigma$ of $\rho_{\mathrm{dm}}=0.3 \mathrm{GeV} / \mathrm{cm}^{3}$, albeit with significant error bars [37]. Despite ever improving measurements of $\rho_{\mathrm{dm}}$ from improved stellar kinematic data and simulations, the DM direct detection community assumes $\rho_{\mathrm{dm}}=0.3 \mathrm{GeV} / \mathrm{cm}^{3}$ for consistency and in order to be able to compare DM results between competing experiments and with previous results.

This density means that many DM particles stream through the detectors every day, with lower mass DM particles generating a higher flux, and direct detection experiments hope to measure this rate of DM events. The expected differential scattering rate, as a function of $E_{\text {recoil }}$, is given by:

$$
\frac{d R}{d E_{\text {recoil }}}=\frac{\rho_{\mathrm{dm}}}{m_{T} m_{\chi}} \int_{v_{\text {min }}}^{\infty} v^{3} f(v)\left[\frac{d \sigma_{\chi T}}{d E_{\text {recoil }}}\left(v, E_{\text {recoil }}\right)\right] d v \quad[\mathrm{keV} \mathrm{kg} \mathrm{day}]^{-1}
$$

where $d \sigma_{\chi T} / d E_{\text {recoil }}$ is the differential cross section, $v_{\min }$ is the minimum WIMP velocity in order to produce recoil energy $E_{\text {recoil }}{ }^{1}$, and $\rho_{\mathrm{dm}}$ is the local dark matter density [40].

Except for the differential cross section $d \sigma_{\chi T} / d E_{\text {recoil }}$ and the WIMP mass, all the parameters of the differential scattering rate are estimated in the SHM. The differential cross section clearly has large implications for the detectability of WIMP particles. The total cross section could be the sum of a spin-independent and spin-dependent term. As Witten and Goodman

\footnotetext{
${ }^{1}$ The revolution of the earth around the sun seasonally adds and subtracts from the WIMP velocity relative to the earth, and detecting a seasonal variation in a possible WIMP signal would be another sign that the signal is indeed the dark matter halo. A different direct detection experiment - the DAMA/LIBRA collaboration-claims that they are seeing this annual modulation in their data and interpret this as a dark matter signal [38]. As shown in Fig. 1.6, multiple other direct detection experiments exclude the DM interpretation of the DAMA/LIBRA data. Additionally, the COSINE-100 experiment searches for DM using the same target (sodium iodide) as DAMA/LIBRA to conduct a model independent test of DAMA's claim; COSINE-100 observes no signal above the expected background and is currently searching for evidence of an annual modulation signal in their data [39].
} 


\subsection{Dark Matter Detection}

noted in their 1984 paper [41], the spin-independent term in the cross section scales as the number of nucleons squared, which is sometimes referred to as "coherent rate enhancement." This effect significantly amplifies the spinindependent term relative to the spin-dependent term, and therefore most direct-detection experiments present their results as a sensitivity to the spinindependent cross section and as a function of the dark matter mass $m_{\chi}$, as shown in Fig. 1.6.

Spin-dependent interactions would occur if the DM particle has a nonzero spin and the target nucleon has a non-zero spin, although the spindependent interaction is more difficult to probe. This is not only because of the lack of coherent rate enhancement, but also because of lack of dark matter nuclei targets with a large nuclear spin. The spin-dependent interaction will cancel for DM scattering off of paired nucleons with equal magnitude but opposite signed spins, and so experiments hoping to detect this interaction use light odd-proton or odd-neutron nuclei (e.g. ${ }^{19} \mathrm{~F}$ as used in the PICASSO experiment [42]) in order to maximize the nuclear spin per unit mass.

We compute the right hand side of Eq. 1.7, and integrate over the $E_{\text {recoil }}$ spectrum to obtain the total number of events expected for different targets and different cross sections as a function of energy threshold. The results are shown in Fig. 1.7. In the left plot we show a relatively light WIMP $\left(10 \mathrm{GeV} / c^{2}\right)$ with a cross section of $\sigma_{S I}=10^{-41} \mathrm{~cm}^{2}$, now ruled out at the $90 \%$ confidence level by a number of experiments. In the right plot we show a $100 \mathrm{GeV} / c^{2}$ WIMP with a cross section of $\sigma_{S I}=10^{-45} \mathrm{~cm}^{2}$, close to the $90 \%$ exclusion limit of the xenon-based dark matter searches [43-45]. As a rough reference, reading off from Fig. 1.7 (left), a recoil threshold of $6 \mathrm{keV}$ gives a rate of $1 / 10$ [events $\mathrm{kg}^{-1} \mathrm{day}^{-1}$ ]. CDMS II had roughly $5 \mathrm{~kg}$ of detector bulk, translating to a rate of 0.5 events per day.

At these relatively low nuclear-recoil energies and low event rates, one fundamental challenge to direct detection experiments is background discrimination. One advantage is that the majority of backgrounds will scatter off electrons in the detector bulk, and most direct detection technologies have means to distinguish electron recoils from nuclear recoils and thus reject background events, which is further discussed in Sec. 2.1.1. In addition, all direct detection experiments shield the detector as best as possible to reduce the background rate. To shield from cosmic-ray muon-induced neutron scattering in the detector, the detectors are operated underground which reduces the muon flux with the rock overburden. The flux reduction for underground sites in which the CDMS detectors have operated is shown in Fig. 1.8. In addition to being underground, the SuperCDMS cryo- 


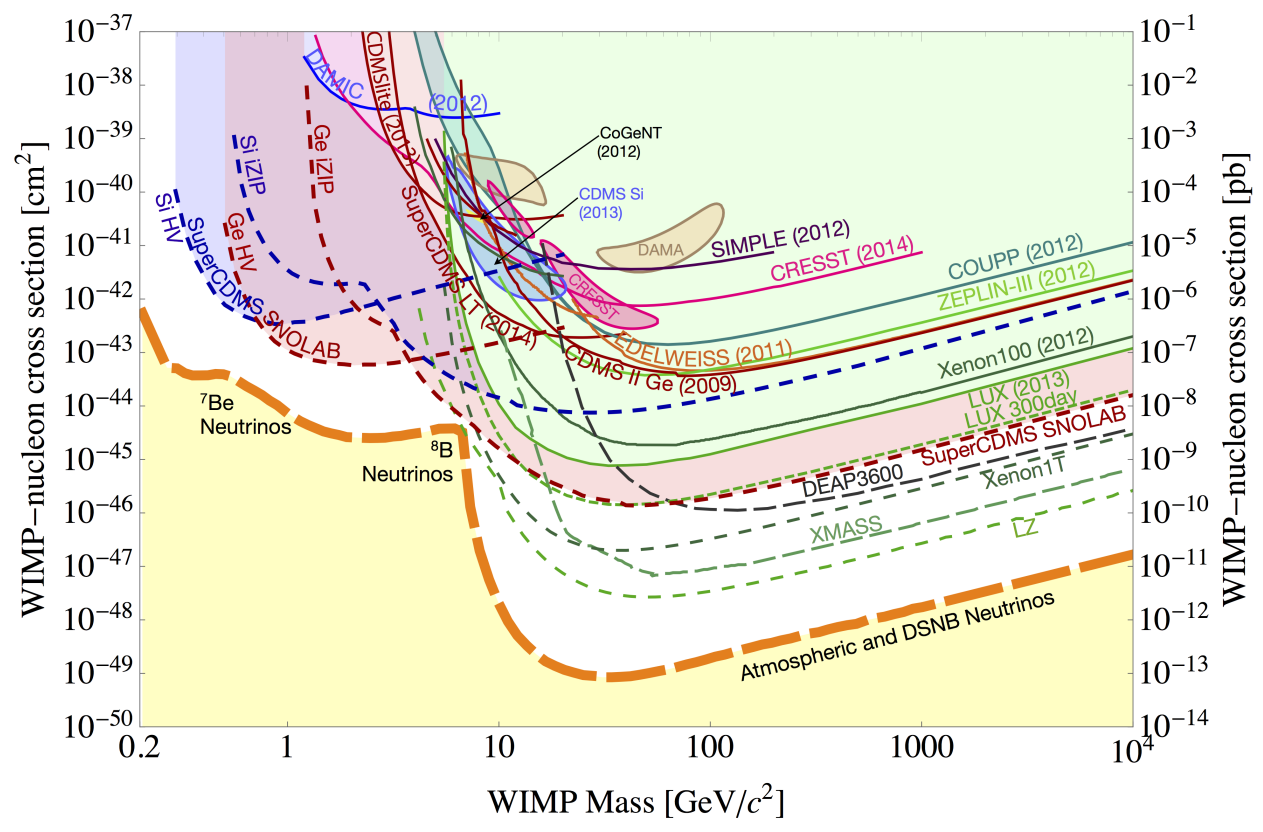

Figure 1.6: Current (solid) and projected (dashed) 90\% limits on the WIMP mass vs. WIMP-nucleon cross section parameter space. The yellow shaded region represents the DM cross section at which experiments will observe an "irreducible" background from nuclear scattering neutrino events. At lower DM mass, the background from solar neutrinos from ${ }^{7} \mathrm{Be}$ and ${ }^{8} \mathrm{~B}$ reactions dominate, whereas at higher DM mass atmospheric and the diffuse supernovae neutrino background (DSNB) dominate. Figure from SuperCDMS collaboration approved public plots. 


\subsection{Dark Matter Detection}
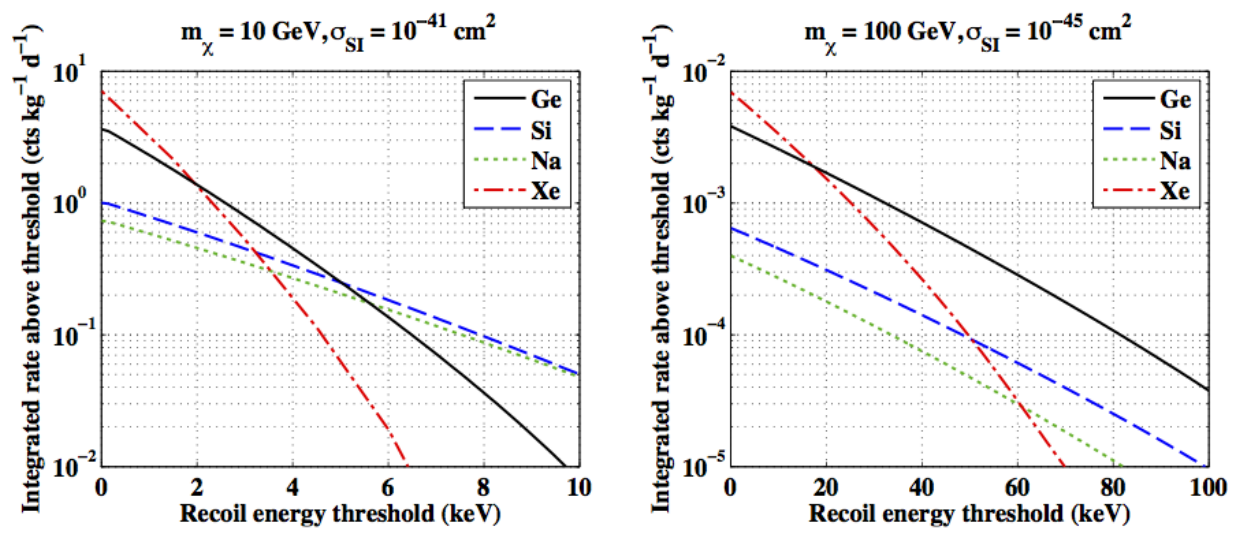

Figure 1.7: The expected WIMP event rate for the given $m_{\chi}$ and spinindependent cross section. $\sigma_{S I}=10^{-41} \mathrm{~cm}^{2}$ corresponds to roughly the cross section reported by DAMA/LIBRA, CRESST, CDMS Si, and CoGent. $\sigma_{S I}=10^{-45} \mathrm{~cm}^{2}$ corresponds to a cross section excluded only by results in the last 2-3 years. Internal CDMS figure, from [46].

stat was surrounded by layers of shielding (polyethylene to block neutrons, ancient lead ${ }^{2}$ to block $\gamma$-rays), and as a result the dominant source of background particles in the detector comes from radioactivity of the shielding itself, other apparatus materials, and the detectors themselves. Finally, most direct-detection experiments have the capacity to estimate the position of an event in the detector, which is useful for fiducialization, or removing background events that occur close to the surface of the detector (at the expense of removing that outer detector volume from the dark matter search as well).

The above is in principle how CDMS and other direct detection experiments hope to discover WIMPs. However, ever since the 1990s when the first limits on WIMP cross sections were published by CDMS, no such rate has been observed. There have been reported detections [38], but they have not held up to further scrutiny and are not widely accepted by the com-

\footnotetext{
${ }^{2}$ Lead on Earth naturally contains some amount of ${ }^{235} \mathrm{U}$, which decays to a radioactive type of lead, ${ }^{210} \mathrm{~Pb}$, which has a half life of 22 years. Most of the uranium is removed when the lead is first processed, and the ${ }^{210} \mathrm{~Pb}$ begins to decay which further purifies the lead over time. In ancient lead almost all of the ${ }^{210} \mathrm{~Pb}$ has already decayed, resulting in radioactive background rates for ancient lead that are $\sim 1000$ times lower than non-ancient lead.
} 


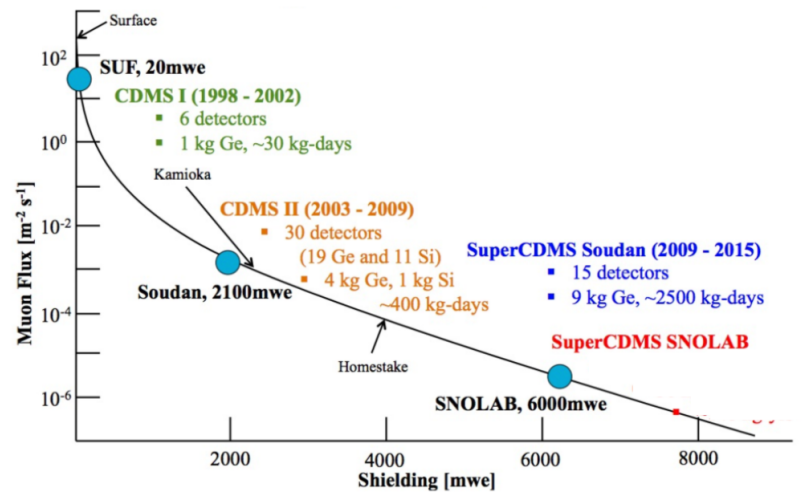

Figure 1.8: The depth of different underground sites, shown as muon flux on the $y$-axis, and meters of water equivalent (mwe) on the x-axis, from different generations of CDMS experiment. SUF stands for Stanford Underground Facility.

munity. CDMS has gone through three generations of experiments: CDMS, CDMS II, and SuperCDMS Soudan, and is now preparing for SuperCDMS SNOLAB. Each generation of the experiment has increased the total detector mass and implemented improved detector technology. In two of the iterations the detectors were moved to a cosmogenically cleaner site (deeper) and radiogenically cleaner environment. Meanwhile competitor experiments made similar improvements and new detection technologies were developed in order to address (1) a low rate of WIMP-nucleon collisions, and (2) background rejection.

\subsubsection{Collider Production}

DM particles could be produced in collisions of leptons and hadrons in high energy colliders. Because the dark matter particles are stable and interact negligibly with the detector, their production in a collision would be observed as a missing transverse momentum.

Both of the general-purpose Large Hadron Collider detectors, ATLAS and CMS, have searched for a DM-like missing momentum in collisions [47, 48]. The data have been consistent with expectations from Standard Model processes and limits have been placed on the DM production rates. In a highly model-dependent fashion, production limits can be converted to limits in the DM-nucleon cross section vs. DM mass plane and be compared with 


\subsection{Dark Matter Detection}
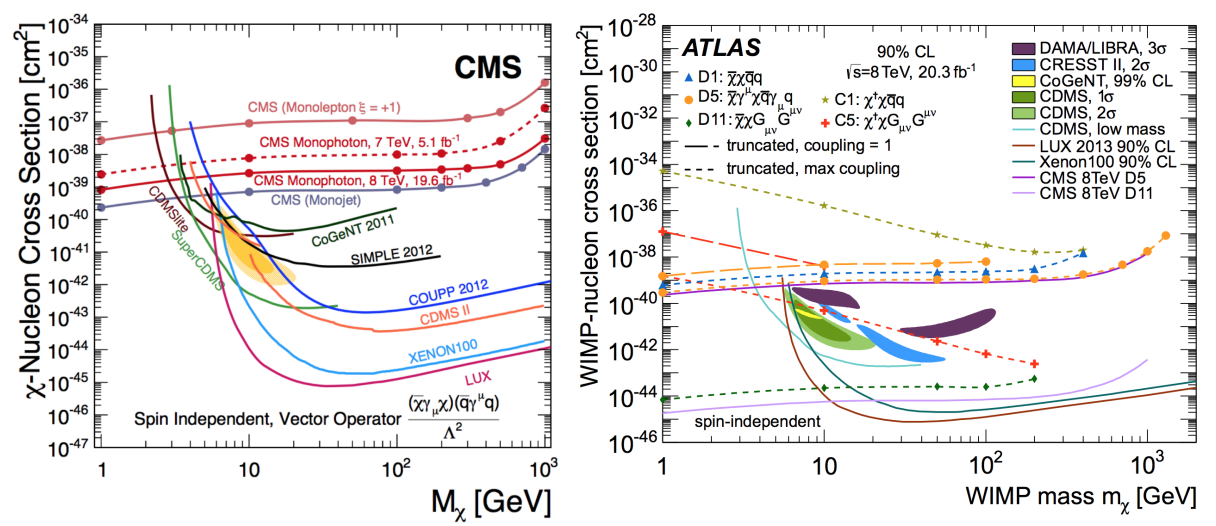

Figure 1.9: Limits on the DM-nucleon scattering cross section, as a function of DM mass from the CMS (left) and ATLAS (right) experiments for different sets of model assumptions. Figures from [47, 48].

direct-detection limits. These conversions are shown in Fig. 1.9. At high DM mass, collider experiments are not as sensitive as the direct detection detectors because they are limited by the energy available in the particle collisions. At low DM mass by contrast, the collider experiments are more sensitive than direct detection detectors because they are not as limited by the low energy thresholds that dictate the mass reach of direct detection experiments.

\subsubsection{Fixed Target}

Fixed target experiments hope to detect dark matter particles by first producing them from proton beam collisions with a "fixed target," and then detecting the dark matter particles in a downstream detector. For example, the MiniBooNE [49] experiment hopes to produce dark matter particles via $8 \mathrm{GeV} / c^{2}$ proton collisions in steel, either by proton Bremsstrahlung or by $\pi^{0}$ or $\eta$ meson (produced copiously in the proton beam interactions with the steel) decay. MiniBoone searches for these dark matter production mechanisms from a "dark photon" kinematically mixing with standard model particles (as mentioned in Sec. 1.5.3). Once the dark matter particles are produced, their elastic nuclear collisions in a downstream detector could be observed. The MiniBooNE detector consists of 818 tons of $\mathrm{CH}_{2}$ mineral oil scintillator, and dark matter nuclear collisions would produce a proton or neutron track that would generate a small, but detectable, amount of 


\subsection{Dark Matter Detection}

scintillation light. In 2018 new limits were placed on the kinematic mixing amplitude for dark matter masses between $10 \mathrm{MeV} / \mathrm{c}^{2}$ and $1 \mathrm{GeV} / c^{2}$ [49]. Many other proposed and ongoing experiments plan similar studies, such as T2K [50] and NO $\nu \mathrm{A}$ [51], and new efforts such as LDMX [52] and HPS [53].

\subsubsection{Indirect Detection}

Indirect detection efforts involve looking for signatures of DM interaction with standard model particles in observable astrophysical objects. One significant challenge in these efforts is accurately modeling of all other non-DM astrophysical processes so that if a DM signal exists in the data it can be correctly attributed as such at a high confidence level.

One channel by which to look for dark matter is its annihilation into gamma rays, e.g. $\chi \bar{\chi} \rightarrow \gamma \gamma$, where $\chi$ is a DM particle and $\bar{\chi}$ is an antiDM particle. Researchers look for gamma ray excesses in regions of the sky where there is predicted to be a high DM density, such as the Milky Way galactic center. The Fermi Large Area Telescope has observed an excess of gamma rays (at roughly $\mathrm{GeV}$ energies) from the galactic center [54], and in some publications this excess has been interpreted as consistent with annihilation of $36-51 \mathrm{GeV} / c^{2}$ DM particles [55]. Subsequent analyses [56, 57] have found that models with unresolved point sources account for the excess and are a better fit to the data, with high statistical significance. The unresolved point sources (e.g. millisecond pulsars) are predicted to generate a gamma flux just below the current Fermi Large Area Telescope point source detection threshold. Lowering of this threshold with future measurements and detecting the point sources will provide additional information about the processes creating the gamma rays in the galactic center.

Another indirect detection method is to look for neutrino excesses from astrophysical bodies where DM particles would gravitationally accumulate, where the probability of their annihilation is higher. One such body is the Sun, and while most of the standard model annihilation products such as gammas would be trapped by the Sun, the neutrinos would escape. Both the IceCube [58] and Super-Kamiokande [59] experiments look for the spectrum of excess neutrinos, and with the analyses indicating that the data are consistent with expected backgrounds they have placed limits on DM annihilation in the Sun. 


\subsection{Further Reading}

While this chapter provides a broad overview of the evidence and search for DM, for a deeper background there are numerous other review articles. Reference [55] contains further discussion of the history of dark matter. Reference [60] reviews DM from an observational and theoretical perspective. Reference [22] establishes and reviews the theoretical motivation for WIMP DM. Reference [36] provides more detailed discussion of the DM rate and energy spectrum expected in terrestrial detectors. 


\section{Chapter 2}

\section{Detectors for the Cryogenic Dark Matter Search}

\subsection{Semiconductor Detector Physics}

The CDMS detectors consist of kilogram-scale cylindrically-shaped ultrapure germanium and silicon crystals. Germanium and silicon have good charge transport properties and small band gaps, which are important for a favorable detector response to a particle interaction in the detector, as discussed in the following sections. In addition, the availability of both germanium and silicon with low concentrations of radioactive contaminants assists in minimizing the number of background events in the detector bulk, which is critical for increasing sensitivity to a DM signal.

The CDMS detectors are operated at low temperatures $(\sim 50 \mathrm{mK})$ at which the germanium and silicon behave as insulators because the $e^{-} / h^{+}$ pairs are frozen out of the conduction band. The low temperature also decreases the heat capacity of the crystal and helps to lower the noise of the readout electronics. When a particle (DM signal or background particle) recoils in the germanium or silicon, it loses energy as it interacts with the electrons and nuclei. For electrons and gammas (i.e. the dominant backgrounds), it is kinematically favorable to interact with and lose energy to the electrons in the detector material. Recoiling heavier particles $\left(\gtrsim 1 \mathrm{GeV} / c^{2}\right)$, such as neutrons or DM WIMPs, find it kinematically efficient to impart significant energy to nuclei in the detector as well as electrons. The detectors exhibit a different response to nuclear recoils (NRs) versus electron recoils (ERs), and historically this enabled discrimination between a DM signal and background events. Because of the importance of these two interaction types in the CDMS detectors, we now discuss some basic dynamics of these two types of recoils. 


\begin{tabular}{l|c|c}
\hline & $E_{\text {create }}$ & $E_{\text {gap }}$ \\
\hline $\mathbf{G e}$ & 3.0 & 0.74 \\
$\mathbf{S i}$ & 3.8 & 1.12 \\
\hline
\end{tabular}

Table 2.1: Electron-hole pair creation energies (in eV) and bandgap energies for germanium and silicon. Values from Ref. [62].

\subsubsection{Electron Recoils}

Electromagnetically-interacting background particles such as $\beta$ and $\gamma$ particles recoil off electrons in the detector bulk. For example, when a medium energy $\gamma$-ray $(10 \mathrm{keV}$ to $1 \mathrm{MeV})$ passes through the detector bulk, it is likely to interact with an electron via the photoelectric effect or by Compton scattering [61]. A recoiling electron will lose energy by Coulomb interactions with other electrons in the material, creating an electronic cascade. The stopping lengths for $\mathrm{keV}$-scale electrons in germanium and silicon are on are the scale of $\mu \mathrm{m}$ and decrease for lower energy electrons, as shown in Fig. 2.1. Because the stopping lengths are significantly smaller than the length scale of the detector, all of the electron's energy will be deposited within the crystal.

In germanium, the energy to create an $e^{-} / h^{+}$pair $\left(E_{\text {create }}\right)$ is $3.0 \mathrm{eV}$. This "ionization energy" is frequently also denoted as $\epsilon$. Therefore, the electronic cascade from a fully absorbed $10 \mathrm{keV} \gamma$-ray spreads its energy out by ionizing $e^{-} / h^{+}$pairs into the conduction band until the individual electrons have insufficient energy to excite another electron, producing $\sim 3000 e^{-} / h^{+}$ pairs in the detector. In general, for an electron recoil, the number of $e^{-} / h^{+}$ pairs is given by

$$
N_{e / h}=E_{r} / E_{\text {create }}=E_{r} / \epsilon
$$

where $E_{r}$ is the energy of the recoil.

With the $e^{-} / h^{+}$pairs in the electronic cascade having on average $3.0 \mathrm{eV}$ of energy, they are unable to lose additional energy to other electrons. However, they have an energy $E_{\text {create }}$ that is greater than the bandgap energy $\left(E_{\text {gap }}=0.74 \mathrm{eV}\right)$ in germanium. Therefore, the $e^{-} / h^{+}$pairs relax down to the bandgap energy and they shed energy as lattice vibrations (i.e. phonons). The corresponding $e^{-} / h^{+}$pair creation energies and bandgap energies for silicon are shown in Table 2.1.

The values of $E_{\text {create }}$ and $E_{\text {gap }}$ dictate the partitioning of the initial recoil energy, of an electron recoil, between the $e^{-} / h^{+}$pairs and the phonons. Specifically, for germanium the fact that the $e^{-} / h^{+}$pairs transfer all of their energy to phonons as they relax from 3.0 to $0.74 \mathrm{eV}$ means that the fraction 


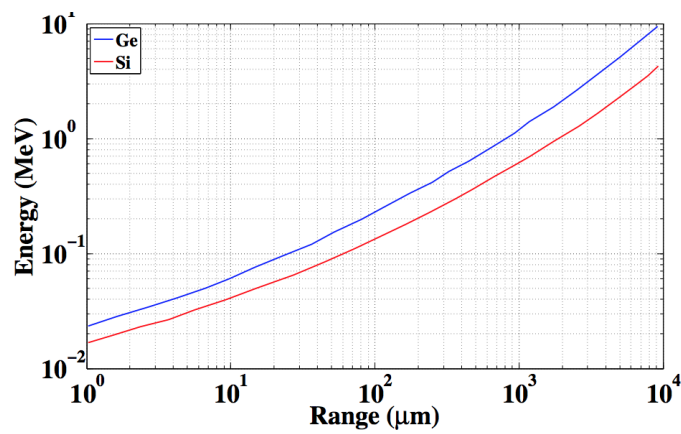

Figure 2.1: Estimated energy vs. electron range in germanium and silicon, based on the electron stopping power from Coulomb interactions. Figure adapted from [61].

of original recoil energy that goes into phonon creation is $1-E_{\text {gap }} / E_{\text {create }}$, or $3 / 4$. For silicon this fraction is $7 / 10$.

\subsubsection{Nuclear Recoils}

WIMP DM particles and neutrons recoil off germanium and silicon nuclei in the detector bulk. Nuclear recoils are similar to electron recoils in many ways; however, they differ most importantly in that a lower fraction of energy goes into $e^{-} / h^{+}$pairs. Lindhard theory, which models the propagation of ions through semiconductors, serves as the basis for the following discussion of semiconductor response to NRs [63-65].

When a nucleus recoils, it is capable of transferring energy to other nuclei and other surrounding electrons. Nuclei are capable of this from a purely kinematic standpoint, whereas electrons are not because of their small mass [66]. The more even division of energy transfer to excitations of electrons versus energy transfer to excitations of other nuclei is particularly true for lower energy $\mathrm{NRs}(\lesssim 1 \mathrm{MeV}$ in $\mathrm{Ge})$, and these low energy recoils are particularly relevant for low-mass DM interactions. The nuclei are freed from the crystal lattice and excite other nuclei in a cascade separate from the electron cascade. Once the nuclei's kinetic energy drops below the lattice binding energy, they have insufficient energy to excite other nuclei and they lose their energy to phonon production. In contrast to the electronic cascade, for nuclei there is no analogous $E_{\text {gap }}$. Therefore, nuclear cascades are more efficient than electron cascades in phonon production.

The derivations of the nuclear recoil energy partitioning between elec- 
trons and phonons are computationally intensive [63-65] and not repeated here, but the Lindhard model provides a parametrization for the fraction of the nuclear recoil energy that ends up in $e^{-} / h^{+}$pairs. This fraction depends on the energy of the nuclear recoil and is called the "ionization yield" (or is sometimes referred to as the "quenching factor"). The ionization yield is normalized so that the ionization yield of electron recoils is equal to 1 . The number of electron-hole pairs generated in a nuclear recoil can be written as a function of the ionization yield $(Y)$ and the nuclear recoil energy $\left(E_{\mathrm{nr}}\right)$ as:

$$
N_{e / h}=\left(Y\left(E_{\mathrm{nr}}\right) \times E_{r}\right) / \epsilon .
$$

The ionization yield as a function of nuclear-recoil energy is predicted by the Lindhard theory to be

$$
Y\left(E_{\mathrm{nr}}\right)=\frac{k \cdot g(\varepsilon)}{1+k g(\varepsilon)}
$$

where $k=0.133 Z^{2 / 3} A^{-1 / 2}, g(\varepsilon)=3 \varepsilon^{0.15}+0.7 \varepsilon^{0.6}+\varepsilon, \varepsilon=11.5 E_{\mathrm{nr}}(\mathrm{keV}) Z^{-7 / 3}$, and $Z$ is the atomic number of the detector material [67]. Measurements of $Y$ in germanium are generally consistent with a small range of $k$ values approximately centered on the Lindhard model prediction of $k=0.159$. Figure 2.2 shows ionization yield (efficiency) as a function of energy for the Lindhard model as well as measurements of the yield in germanium [68-72]. Section 5.2 discusses how the spread in experimental measurements is incorporated as a systematic uncertainty on $k$ whenever using the Lindhard model to determine how a nuclear recoil signal would appear in a CDMS detector.

\subsubsection{Yield Discrimination}

The fact that NRs produce fewer $e^{-} / h^{+}$pairs, and therefore a smaller ionization signal, than ERs of the same recoil energy offers a powerful means of discriminating between the two types of events. CDMS detectors measure the ionization yield and the recoil energy for each event independently by measuring the signal from both the $e^{-} / h^{+}$and the phonons.

The expected difference in ionization yield between ERs and NRs is observed in ${ }^{133} \mathrm{Ba}$ and ${ }^{252} \mathrm{Cf}$ calibration data that induce the two different types of recoils, and is shown for a CDMS II detector ${ }^{3}$ in Fig. 2.3. The ER

\footnotetext{
${ }^{3}$ CDMS II was a generation of the experiment that operated from 2003-2009.
} 


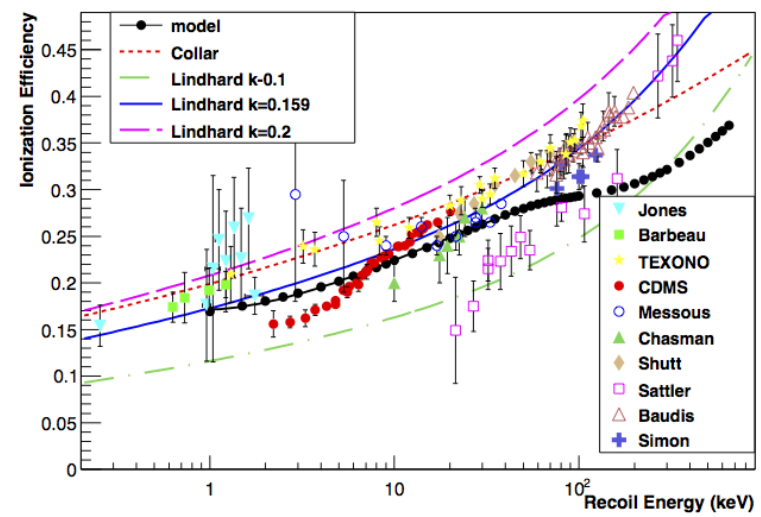

Figure 2.2: Measurements of the ionization yield of nuclear recoil events, as a function of energy. The prediction from the Lindhard model is overlaid. From Ref. [73].

background events can be removed by only selecting events with lower ionization yield. The upper (lower) $\pm 2 \sigma$ band in Fig. 2.3 defines the ER (NR) region of parameters space, where the width of the bands is determined by the energy resolution of the $e^{-} / h^{+}$and phonon measurements. The resolution of these measurements worsens as the energy decreases, demonstrated by the widening of the bands at lower energy, and the specifics of the measurements is discussed in Sec. 2.2. Deciding where to "set the cut" in the ionization yield vs. recoil energy plane is analysis- and detector-dependent (some complications of which are discussed in Sec. 2.4), though the lower $\pm 2 \sigma$ bands shown in Fig. 2.3 depict a reasonable selection area for NRs.

\subsubsection{Phonon Generation and Propagation}

While Sec. 2.1.1 and 2.1.2 outlined electron and nuclear recoil dynamics, special attention should be given to the different production mechanisms of phonons in the detector. Phonon production and amplification is a particularly important aspect of the CDMSlite detector operation, which is the focus of Chapter 4 of this thesis. Phonons are produced at three different stages of an event, as depicted in Fig. 2.4, and we describe each stage in more detail below.

The phonons produced in the initial electron or nuclear recoil cascade are referred to as prompt phonons. These prompt phonons are high frequency 


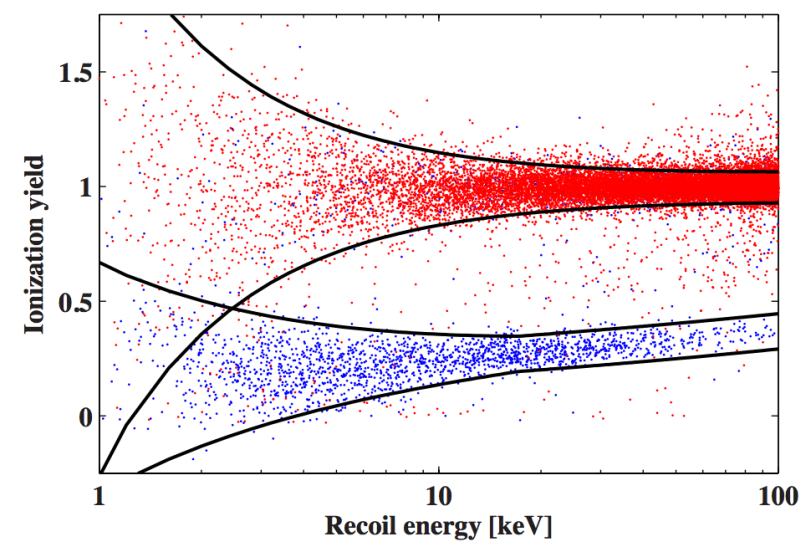

Figure 2.3: Ionization yield versus recoil energy for calibration data, from a CDMS II detector. The blue data was acquired during exposure of the detector to a neutron ${ }^{252} \mathrm{Cf}$ source producing primarily NRs, which accordingly have a measured ionization yield less than 1 . The red data is from a ${ }^{133} \mathrm{Ba}$ calibration, which primarily consists of ERs from $\gamma$ interactions in the detector. The black bands correspond to the $\pm 2 \sigma$ area in the ionization yield vs. recoil energy plane where ERs and NRs occur. These bands provide a visualization of the discrimination in this plane, and a cut in this plane would be customized for a specific analysis (not necessarily at the $\pm 2 \sigma$ level). From Ref. [46].

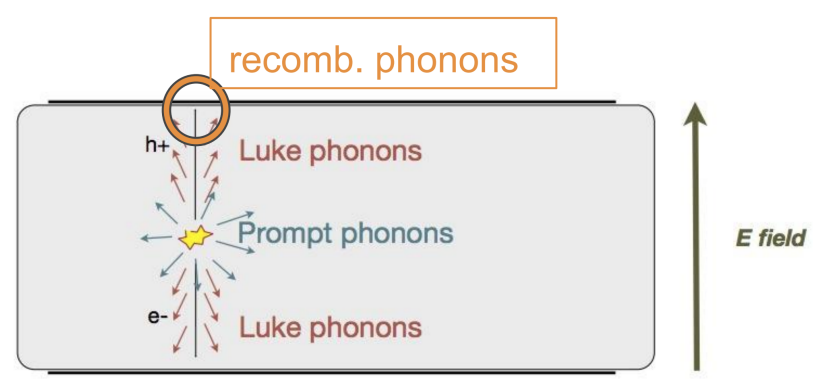

Figure 2.4: A simple depiction of an event in the detector and the resulting prompt, NTL (or Luke), and recombination phonon production. 
$(\nu>1 \mathrm{THz})$ and their total energy is given by:

$$
E_{P}=E_{r}-N_{e / h} E_{g a p} .
$$

Two frequency-dependent scattering processes - anharmonic decay (with a scattering rate, $\Gamma_{A}$, proportional to $\left.\nu^{5}\right)$ and isotope scattering $\left(\Gamma_{B} \propto \nu^{4}\right)$ downconvert the initial high frequency phonons into lower frequency phonons. The initial prompt phonons scatter with mean free paths that are much smaller than the size of the detector, but once they reach frequencies of $\nu \sim 2 \mathrm{THz}$, the decrease in the scattering rates leads to intermediate mean free paths of $10 \mu \mathrm{m}-1 \mathrm{~cm}$ [74]. This intermediate propagation regime is referred to as "quasi-diffusive," and the diffusive phonon ball that spreads out from the recoil site carries information about the position of the event. Over timescales of $\sim 100 \mu \mathrm{s}$, the quasi-diffusive phonons continue to downconvert to lower frequency, which in turn lowers the scattering rate and increases the mean free path to length scales comparable to the size of the detector. This propagation regime is referred to as ballistic, where the distribution of phonons is homogenous through the detector and carries no position information about the initial recoil. These phonons bounce around the detector, reflecting off the detector surfaces, and are absorbed with some probability when they strike an aluminum collection fin (discussed in Sec. 2.2.2).

A second production mechanism of phonons adds to the total phonon signal after the initial electron or nuclear recoil. A voltage is applied across the detector so that once an electron cascade has occurred, the excited $e^{-} / h^{+}$pairs do not de-excite back into valence states, but rather drift toward the surfaces of the detector. As $e^{-} / h^{+}$pairs drift across the crystal due to the electric field, they quickly reach a terminal velocity in the crystal and the additional work done on the carriers is transferred to the crystal lattice in the form of Neganov-Trofimov-Luke (NTL) phonons [75, 76]. For a single $e^{-} / h^{+}$pair generated in the bulk of the detector, the work done to drift the carriers to the surface of the detector by a distance $d$ is given by

$$
W_{q}=e|\mathbf{E}| d=e V_{\mathrm{b}},
$$

where $e$ is the absolute value of the charge of the electron, $\mathbf{E}$ is the electric field through the detector, and $V_{\mathrm{b}}$ is the voltage difference between the surfaces of the detector ${ }^{4}$. With the number of $e^{-} / h^{+}$pairs generated in an

\footnotetext{
${ }^{4}$ Non-uniformities in the electric field through the detectors, which complicate the relationship given by Eq. 2.5, are discussed in Sec. 2.5.
} 
electron or nuclear recoil given by Eq. 2.1 and 2.2 respectively, the total energy in the NTL phonons is given by

$$
E_{\mathrm{NTL}}=e V_{\mathrm{b}} N_{e / h}
$$

A third class of phonons, called recombination phonons, also contribute to the total phonon signal. Once the $e^{-} / h^{+}$pairs reach the surface of the detector they recombine at the charge collection electrodes (discussed in Sec. 2.2.1) and the bandgap energy of the charge carriers is converted into phonons. The energy of the recombination phonons is given by

$$
E_{R}=E_{\text {gap }} \times N_{e / h} .
$$

There is some uncertainty in the detector's collection efficiency for the recombination phonons. The possibility of reduced collection efficiency of this type of phonon, relative to prompt and NTL phonons, is discussed further in the context of bias scan calibration data (Appendix A.1).

The total phonon energy $\left(E_{t}\right)$ is nominally the sum of these three sources (prompt, NTL, and recombination), given by

$$
E_{t}=\left(E_{r}-N_{e / h} E_{g a p}\right)+\left(e V_{\mathrm{b}} N_{e / h}\right)+\left(E_{\text {gap }} N_{e / h}\right),
$$

which simplifies to

$$
E_{\mathrm{t}}=E_{\mathrm{r}}\left(1+\frac{Y\left(E_{r}\right) e V_{\mathrm{b}}}{\epsilon}\right)
$$

In comparing the energy contribution of prompt (Eq. 2.4), NTL (Eq. 2.6), and recombination (Eq. 2.7) phonons to the total phonon energy (Eq. 2.8), notice that for low detector voltage $V_{\mathrm{b}}$, the prompt and recombination phonons dominate. However, once $V_{\mathrm{b}}=3$ volts the NTL phonon contribution makes up half of the total phonon signal (for electron recoils). In the CDMSlite operating regime of $V_{\mathrm{b}}=75$ volts, the NTL phonon energy dominates the total energy, with 25 times more energy than the prompt and recombination phonons.

\subsection{Amplifiers}

The detector surfaces are instrumented with sensors designed to measure the $e^{-} / h^{+}$pairs and phonon energy such that the energy, ionization yield, and position of an event can be estimated as accurately as possible. As energy 

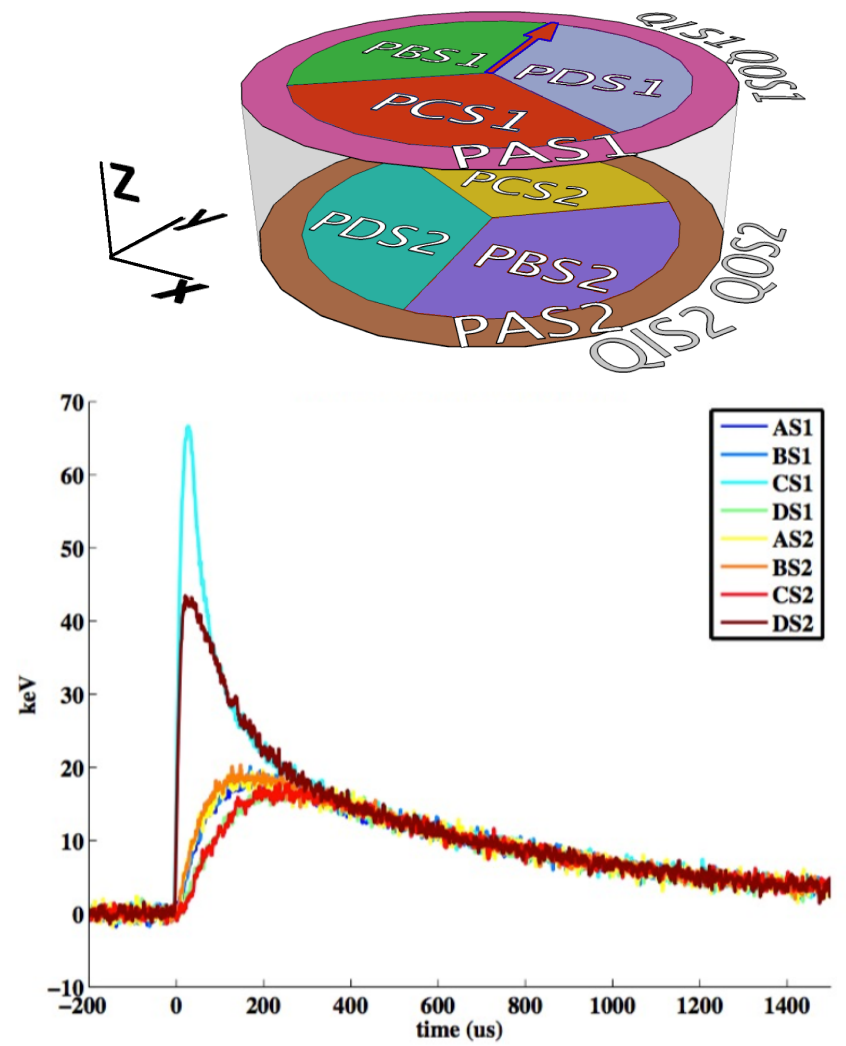

Figure 2.5: (top) A cartoon of the iZIP detector, with the individual phonon channels labeled and uniquely colored. (bottom) A characteristic phonon signal (i.e. pulse) from the bulk of an iZIP detector. The different pulse shapes on the individual channels indicate that this event occurred close to channel DS2 and CS1. 
2.2. Amplifiers

\begin{tabular}{|l|c|c|c|}
\hline & $\begin{array}{c}\text { CDMS } \\
\text { II }\end{array}$ & $\begin{array}{c}\text { SCDMS } \\
\text { Soudan }\end{array}$ & $\begin{array}{c}\text { SCDMS } \\
\text { Soudan }\end{array}$ \\
\hline Detector & ZIP & iZIP & CDMSlite \\
\hline Mass per Detector $[\mathrm{kg}]$ & 0.25 & $\sim 0.62$ & $\sim 0.62$ \\
\hline Number of Detectors & 19 & 15 & 2 \\
\hline Phonon Channels per Det. & 4 & 8 & 4 \\
\hline Phonon Energy Res. [eV] & $\sim 180$ & $\sim 200$ & $\sim 70$ \\
\hline Trigger Threshold [eV] & $\sim 2000$ & $\sim 3000$ & $\sim 50$ \\
\hline Charge Energy Res. $[\mathrm{eV}]$ & $\sim 300$ & $\sim 450$ & - \\
\hline Voltage between faces $[\mathrm{V}]$ & 3 & 4 & $\sim 70$ \\
\hline
\end{tabular}

Table 2.2: Detector amplifier specifications for different generations of the germanium CDMS detectors. Adapted partially from Ref. [66].

resolutions improve, event discrimination improves and detector thresholds can be lowered. As position resolutions improve, radioactive backgrounds that occur near the surface of the detector can be removed more efficiently. All of these factors improve sensitivity to a DM signal.

With R\&D advances in sensor design, the resolutions of CDMS detectors have improved over time, and different generations of CDMS detectors employed different sensor layouts. Table 2.2 provides some information on the amplifier specification for three different generations of CDMS detectors. We specifically list the "iZIP" and "CDMSlite" style detectors used in the SuperCDMS Soudan generation of the experiment, which we discuss in more detail in Sec. 2.4 and Sec. 2.5. The DM search analysis described in Chapter 4 of this thesis used the "CDMSlite" style detectors. We also list the "ZIP" detector from the earlier CDMS II generation of the experiment.

\subsubsection{Measuring the $e^{-} / h^{+}$Energy}

As described previously, a voltage is applied across the CDMS detectors so that the $e^{-} / h^{+}$pairs generated in an event do not recombine at the recoil site but rather drift to the detector faces. As the ionization drifts to the surface, image charges are induced on the top and bottom faces of the detector. In CDMS II and SuperCDMS detectors, FET (Field Effect Transistor) amplifiers read out the image charge on the electrodes and amplify this signal as a voltage that is further amplified by downstream amplifiers and eventually digitized as a function of time by the data acquisition system.

In the CDMS II ZIP and SuperCDMS iZIP detectors, the voltage has been tuned to the smallest possible value such that the $e^{-} / h^{+}$pairs drift 
across the full length of the detector. If the potential difference between detector faces is insufficiently high, the the $e^{-} / h^{+}$pairs can trap in local minima created by impurities in the crystal. At the same time, if the potential difference between detector faces is too high, the discrimination between ERs and NRs suffers because the NTL phonons correlate the $e^{-} / h^{+}$pair signal with the recoil phonon signal. Therefore, for ER vs. NR discrimination, the optimal field was found to be $\sim 1 \mathrm{~V} / \mathrm{cm}$; for the CDMSlite approach where the detector is operated at greater than $70 \mathrm{~V}$, ER vs. NR discrimination is sacrificed in order to amplify the signal to achieve a lower threshold.

The propagation time of $e^{-} / h^{+}$pairs to the surface of the detectors is $\mathcal{O}(n s)$, the amplifier is "faster" than the propagation time (i.e. responds to a signal on timescales $<1 \mathrm{~ns}$ ), and the digitization rate of the amplifier signal is $0.8 \mu \mathrm{s}$; therefore, the risetime of the $e^{-} / h^{+}$pair signal is sharp and contained in 1 or 2 time digitization bins. The falltime of the $e^{-} / h^{+}$pair signal is controlled by electronic components of the FET amplifier, and this falltime was selected to be significantly longer than the charge collection time; therefore the $e^{-} / h^{+}$pair signal pulse assumes a relatively constant shape, regardless of the location of the initial recoil [77].

While the pulse shape of the $e^{-} / h^{+}$pair signal does not contain information about the position of the event, the electrodes are divided up into a number of channels, and the relative amplitude of the charge signal in those channels does provide position information. For example, the iZIP detector design employs a specific electrode channel layout to identify events that occur near the surface of the detector, and this feature is described further in Sec. 2.4.

\subsubsection{Measuring the Phonon Energy}

Phonon channels are instrumented on the detector faces, and each channel consists of thousands of Transition Edge Sensors (TESs). The TESs are made from tungsten superconducting material whose transition temperatures $\left(T_{c}\right)$ are tuned in fabrication to be at cryogenic temperatures, but above the base temperature of the cryostat (i.e. the temperature of the detectors). CDMS has fabricated TESs with $T_{c}$ between $30 \mathrm{mK}$ and $200 \mathrm{mK}$. The voltage-biased TESs are held within the range of their transition such that when heat from phonons reaches the sensors their resistance changes rapidly and the current through them decreases. The current through the TES is inductively coupled to a Superconducting Quantum Interference Device (SQUID) which further amplifies the reduction in current. TESs are amongst the most sensitive phonon amplifiers in existence, and they are 
at the heart of the CDMS detectors' sensitivity to dark matter. The sensor technology is reviewed in K. Irwin's and G. Hilton's review article (Ref. [78]).

Significantly more information than just the phonon energy is encoded in the signal readout from the TESs. The prompt phonons in the first $\sim 100 \mu \mathrm{s}$ impart more power on the sensors closest to the event. In contrast, the NTL and ballistic phonons carry no position information, but do provide the best measure of the event energy. The total absorption time of the ballistic phonons is dependent on the aluminum collection fin coverage of the detector, and this affects the falltime of the phonon signal.

The falltime of the phonon signal is roughly linearly dependent on the area of the coverage, and the iZIP detector's $\sim 5 \%$ coverage leads to relatively long $750 \mu \mathrm{s}$ falltimes. The design decision of relatively low aluminum coverage led to some advantageous detector response characteristics, namely a straightforward method for separating event position from event energy in the different time scales of the phonon pulse. Figure 2.5 offers a visualization of the phonon propagation and collection in the iZIP detectors. A position-dependent signal exists in the first $\sim 100 \mu$ s of the pulses, from the prompt phonons, with much larger signals on channel DS2 and CS1. After $\sim 300 \mu$ s the phonons are in the fully ballistic regime and impart equal power to each phonon channel, as shown by the aligned "tails" of the pulses at later times. We obtain an estimate of the event location by comparing the energy deposited in the different phonon channels. The partitioning of energy in the different channels allows a weighted average to determine the $\mathrm{x}, \mathrm{y}$, and $\mathrm{z}$ coordinate of the initial event within the detector[77]. The pulse shape of the phonon pulse (e.g. the peakiness of the pulse) in the individual channels is also used to obtain more information about the event position and energy. For example, in the analysis described in Sec. 4 of this thesis, it was particularly important to use phonon pulse shape to estimate the radial position of the event in the detector. This estimation is described in Sec. 4.8 .

\subsection{Optimal Filter Event Reconstruction}

Following the discussion of the techniques used to measure $e^{-} / h^{+}$and phonon energy, it is important to discuss the techniques used to fit and extract information from the raw data. For every event, the raw data consists of a time stream of data, read out for each channel, that has been digitized from the amplifiers. We list the digitization rates and other important time and frequency values in Table 2.3. Because the analysis in this 
2.3. Optimal Filter Event Reconstruction

\begin{tabular}{c|c|c|c|c|c} 
& $R(\mathrm{kHz})$ & $N$ & $T(\mathrm{~ms})$ & $\Delta f(\mathrm{~Hz})$ & $\Delta t(\mu \mathrm{s})$ \\
\hline Charge & 1250 & 2048 & 1.6384 & $\sim 610.4$ & 0.8 \\
Phonon & 625 & 4096 & 6.5536 & $\sim 152.6$ & 1.6
\end{tabular}

Table 2.3: Important time and frequency values for charge $\left(e^{-} / h^{+}\right)$and phonon amplifier digitizers from SuperCDMS Soudan. $R, N, T, \Delta f, \Delta t$ refer respectively to the digitization rate, the number of bins in the read out time stream, the time length of the read out time stream, the lowest resolvable frequency $(1 / T)$, and the time length of one digitization bin.

thesis focuses on the SuperCDMS Soudan generation of the experiment, we list the digitization rates used in that generation, though the digitization rates do not change much between generations.

After the amplifier signals are digitized and saved, we use fitting algorithms to extract information from these raw data. For example, for the raw data pulse in Fig. 2.6 (left), we would like to estimate the amplitude of the pulse, the time at which the pulse occurs, the "peakiness" of the first $\sim 500 \mu$ s of the pulse, as well as a $\chi^{2}$ parameter that indicates the goodness of fit. We primarily rely on three different types of "optimal filter" (OF) algorithms to fit the raw data pulses and extract these pieces of information.

\subsubsection{Simple 1D Optimal Filter}

In this section we explain the basics of optimal filter (OF) theory. Using Fig. 2.6 for visualization, the $\mathrm{OF}$ is a fit of a template (red) to the data (blue) to determine the amplitude and start time (i.e. delay) of the pulse in the data. The OF is truly the optimal algorithm to determine the amplitude and delay under a set of assumptions: (1) the template perfectly describes the shape of the underlying pulse in the data, and (2) the noise is a Gaussian random process whose power spectral density (PSD) is known. Even when these two characteristics aren't strictly realized in the real data, the optimal filter fits perform well.

For Gaussian random noise, the frequency components of the noise are uncorrelated. This is why it is beneficial to perform the fit in the frequency domain - an equivalent fit in the time domain would require accounting all covariances between the time domain data points. The optimal filter performs the fit in the frequency domain where each frequency can be weighted by the signal-to-noise ratio, effectively extracting the signal from the noise to get the best estimate of amplitude and delay. We will use Jeff Filippini's 


\subsection{Optimal Filter Event Reconstruction}
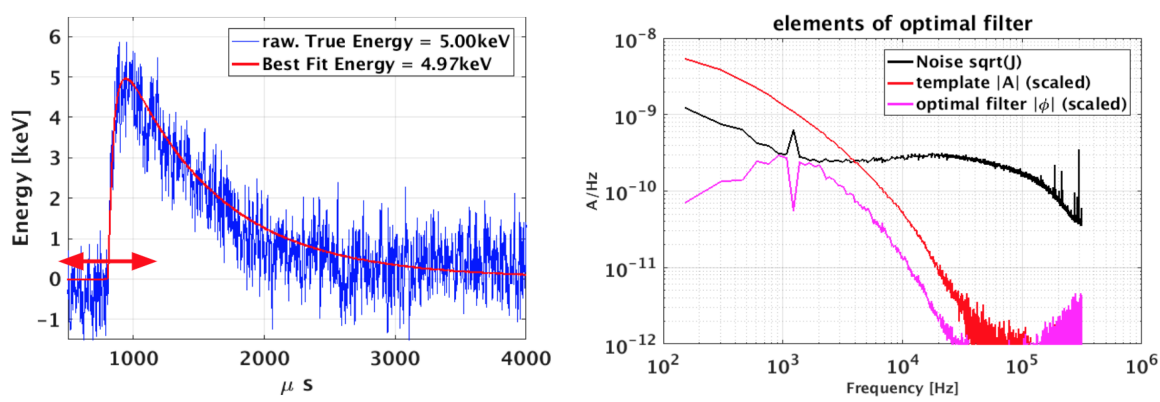

Figure 2.6: (left) An example simulated pulse with the template (normalized to the best fit amplitude as given by the optimal fit). The template is also left-right aligned with the best fit time delay as given by the optimal fit. (right) The frequency domain representation (magnitude only) of the template, noise (PSD), and optimal filter.

notation from Appendix A of his thesis, where $A$ is the template, $S$ is the signal, and $J$ is the noise PSD [77]. A discussion of how to calculate the noise PSD is provided in Sec. 3.2. Additionally, the $n$ subscript represents the Fourier index, and $\tilde{A}$ and $\tilde{S}$ are the Fourier transforms of the template and signal, respectively.

The $\chi^{2}$ for the fit of the signal amplitude, $a$, is given by:

$$
\chi^{2}(a)=\sum_{n}^{N} \frac{\left|\tilde{S}_{n}-\tilde{A}_{n}\right|^{2}}{J_{n}}
$$

and the value of $a$ that minimizes this $\chi^{2}(\hat{a})$ can be solved for analytically and is given by

$$
\hat{a}=\sum_{n} \frac{\tilde{A}_{n}^{*} \tilde{S}_{n}}{J_{n}} / \sum_{n} \frac{\left|\tilde{A}_{n}\right|^{2}}{J_{n}} .
$$

Typically, as suggested by the $\chi^{2}$ in Eq. 2.10, the optimal filter is thought of in the context of a fit to a pulse; however, Eq. 2.11 shows that the optimal filter can also be thought of applying a filter to the data. The denominator of Eq. 2.11 is simply a normalization constant (that is, it is independent of the data $\tilde{S}_{n}$ ), and so the numerator is the application of a Fourier domain filter $\tilde{\phi}$, given by

$$
\tilde{\phi_{n}}=\frac{\tilde{A}_{n}^{*}}{J_{n}}
$$




\subsection{Optimal Filter Event Reconstruction}

to the Fourier transform of the signal. A visualization of the amplitude of this filter is shown in Fig. 2.6 (right), where it is clear that any particularly noisy frequency $(1.2 \mathrm{kHz}$ in this mock example) is deweighted in the optimal filter $\tilde{\phi}_{n}$.

The best fit amplitude of the signal, given by $a$, and the time offset or delay, given by $t_{0}$, are obtained by minimizing the following $\chi^{2}$ :

$$
\chi^{2}\left(a, t_{0}\right)=\sum_{n}^{N} \frac{\left|\tilde{S}_{n}-a e^{-2 \pi i t_{0} f_{n}} \tilde{A}_{n}\right|^{2}}{J_{n}}
$$

which has used the fact that a shift in the time domain $\left(A\left(t-t_{0}\right)\right)$ corresponds to a phase rotation in the frequency domain $\left(\tilde{A}(f) e^{-2 \pi i t_{0} f}\right)$. This $2 \mathrm{D} \chi^{2}$ can be minimized analytically, and therefore OFs can be performed efficiently, with the limiting computational step being a fast Fourier transform [77].

We frequently refer to best fit quantities from the 1D OF algorithm with the "OF" suffix. For example, "ptOF" refers to the energy of the total phonon pulse (the sum of the phonon pulses on all the detector channels) as estimated by the fitted amplitude from the standard 1D OF algorithm.

\subsubsection{Non-Stationary Optimal Filter}

In the previous section, we described how the OF formalism was only optimal if the template perfectly described the underlying shape of the pulse in the data. However, in Sec. 2.4, we described the position-dependent pulse shapes in the iZIP detectors. The non-stationary optimal filter improves the energy resolution of the $1 \mathrm{D}$ OF algorithm by treating the pulse shape non-uniformity as a source of non-Gaussian, non-stationary noise ${ }^{5}$. The insight of treating the position dependence of the signal as noise came from M. Pyle [79]. The non-uniform pulse shape of $10 \mathrm{keV}$ events in the CDMSlite Run 3 detector is shown in Fig. 2.7 (left) with the residuals shown in Fig. 2.7 (right). The position-dependent pulse shape variation noise between bin 500 and 600 is clearly highly correlated across time and frequencies. This introduces a complication because, in considering the non-stationary noise, the frequency domain no longer exhibits the convenient noise orthogonality assumed in Eq. 2.16 for a 1D optimal filter. Therefore the non-stationary

\footnotetext{
${ }^{5}$ The term "non-stationary" describes a type of noise whose variance changes in time.
} 


\subsection{Optimal Filter Event Reconstruction}
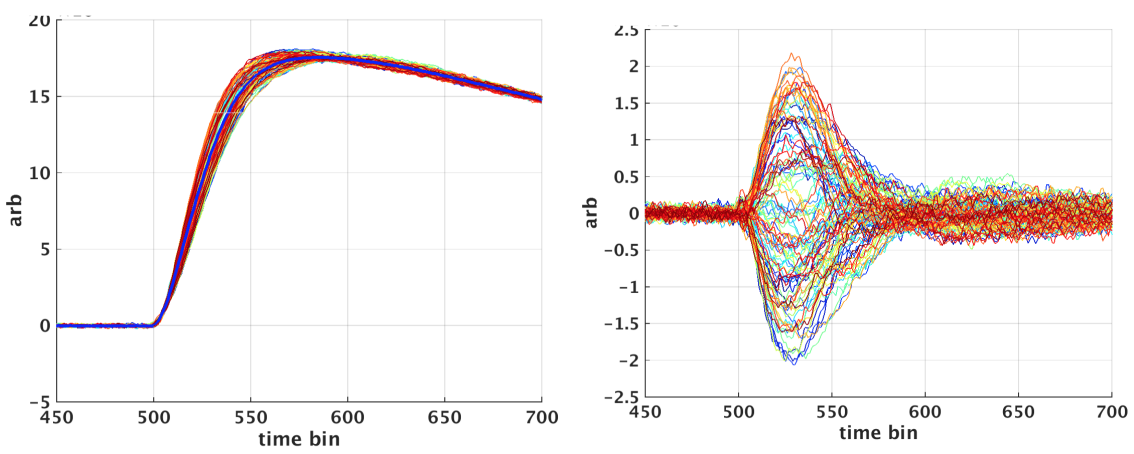

Figure 2.7: (left) Example $10 \mathrm{keV}$ total phonon pulses with the OF template overlaid in blue. (right) The residuals (phonon pulse - template) showing that the position-dependent part of the pulse can be treated as a non-stationary source of noise.

OF algorithm promotes the noise PSD $J$ to a matrix $\tilde{\mathbf{V}}$ :

$\chi^{2}\left(a, t_{0}\right)=\sum_{f, f^{\prime}}\left(\tilde{S}^{*}(f)-a \tilde{A}^{*}(f) e^{2 \pi i t_{0} f}\right)\left(\tilde{\mathbf{V}}\left(f, f^{\prime}\right)^{-1}\right)\left(\tilde{S}\left(f^{\prime}\right)-a \tilde{A}\left(f^{\prime}\right) e^{-2 \pi i t_{0} f^{\prime}}\right)$.

and the covariance between frequency modes is included when this $\chi^{2}$ is minimized.

The covariance matrix $\tilde{\mathbf{V}}$ is determined using the Fourier transforms of the pulse residuals (the phonon pulse minus the best fit phonon template) shown in Fig. 2.7. The complete non-stationary optimal filter algorithm also accounts for the fact that the magnitude of the stationary noise is independent of the pulse energy but the non-stationary noise increases as a function of the pulse energy, and therefore the off-diagonal components of $\tilde{\mathbf{V}}$ undergo a magnitude scaling as a function of the pulse energy. The details of this implementation are beyond the scope of this discussion, and further details can be found in Ref. [80].

We frequently refer to best fit quantities from the non-stationary OF with the "NF" suffix. For example, "ptNF" refers to the energy of the total phonon pulse as estimated by the non-stationary OF algorithm. 


\subsection{Optimal Filter Event Reconstruction}

\subsubsection{Two Template Optimal Filter}

In contrast to the position-dependent deweighting approach of the nonstationary $\mathrm{OF}$, the two-template $\mathrm{OF}$ seeks to fit the position-dependent part of the pulse with two templates and then use the best template fit amplitudes and delays to extract enhanced energy and position information about the event. Motivated by the prompt vs. ballistic phonon propagation dynamics discussed in Sec. 2, the two template OF posits that the signal is a linear superposition of two different pulses each with different time constants. A "fast" template (with a fast falltime) is used in addition to the standard "slow" template, such that we can describe the signal pulse as

$$
S(t)=a_{1} A_{1}\left(t-t_{0}\right)+a_{2} A_{2}\left(t-t_{0}\right)+n(t)
$$

where the noise term $n(t)$ is modeled as Gaussian noise. The slow template $\left(A_{1}\right)$ is identical to that used in the $1 \mathrm{D}$ OF and the non-stationary OF. Then the signal is fit with the fast and slow template, and Fig. 2.8 shows examples of such fits. Computationally this is relatively straightforward, where the $\chi^{2}$ rewritten with new index $j=1,2$ for the two templates is

$$
\chi^{2}\left(a_{1}, a_{2}, t_{0}\right)=\sum_{n}^{N} \frac{\left|\tilde{S}_{n}-a_{1} e^{-2 \pi i t_{0} f_{n}} \tilde{A}_{1, n}-a_{2} e^{-2 \pi i t_{0} f_{n}} \tilde{A}_{2, n}\right|^{2}}{J_{n}}
$$

and the $\chi^{2}$ is minimized for $t_{0}$ and the two amplitudes, $a_{1}$ and $a_{2}$.

We frequently refer to best fit quantities from the two template OF with the "2T" suffix.

\subsubsection{Use of Different OF Algorithms}

For the Run 3 analysis, based on the analysis task being performed, we use the parameter(s) derived from one of the three different OF algorithms. The different optimal filters used for the different analysis tasks is given in Table 2.4. After applying the corrections discussed in Sec. 4.4.1, the energy resolution of the non-stationary OF is superior to that of the other two algorithms, and therefore we use the non-stationary OF algorithm for energy estimates. As discussed in Sec. 4.8, the parameters derived from the two template OF are superior to other OFs for determining the position of the event in the detector. Information from the standard $1 \mathrm{D} \mathrm{OF}$ is used to distinguish good events from instrumental background events. 

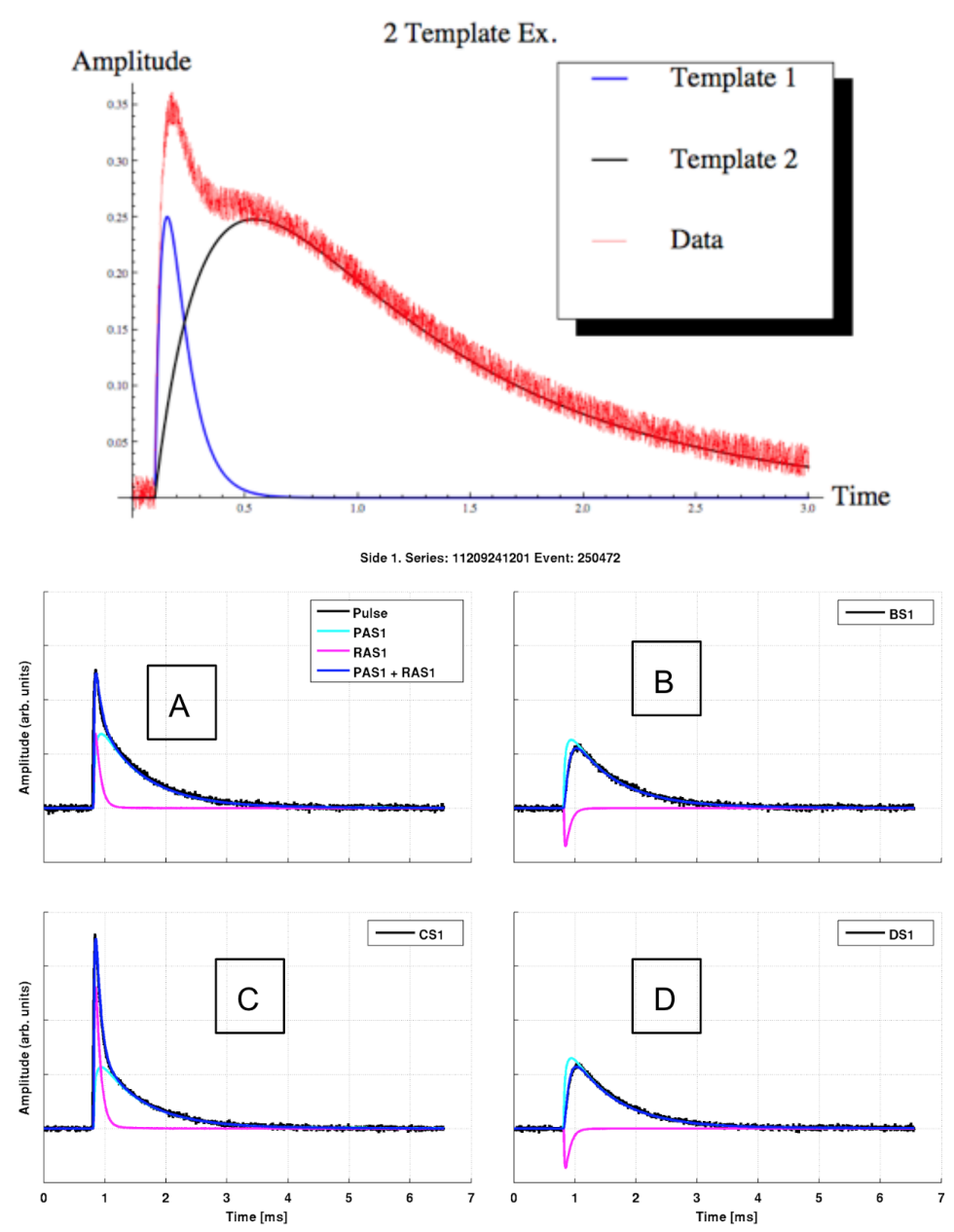

Figure 2.8: (top)An example of the 2 template fit to data, where the prompt signal is modeled by the fast template and the ballistic signal is modeled by the slow template. (bottom) An example of the 2 template fit performed on the 4 individual phonon channels for a single event on a CDMSlite detector. 
2.4. iZIP Interleaved Design

\begin{tabular}{l|r}
\hline OF Type & Primary Use \\
\hline simple 1D & instrumental background discrim. \\
non-stationary & energy estimate \\
two template & position estimate \\
\hline
\end{tabular}

Table 2.4: The primary use of the three different OF algorithms.

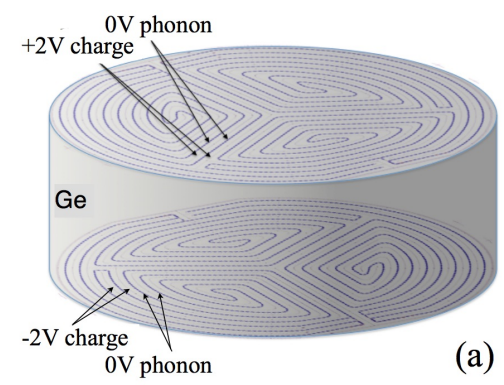

Figure 2.9: The iZIP design with interleaved charge electrodes $( \pm 2 V)$ and phonon rails (0V). Used with permission, from [81].

\section{4 iZIP Interleaved Design}

The CDMS II detectors suffered primarily from one design flaw. The ionization from electron recoils close to the detector surface was more prone to trapping, which reduced the ionization yield of the event [81]. This reduced ionization yield caused surface electron recoils to mimic the nuclear recoil signature and thus leak into the WIMP signal region. CDMS II sensitivities were limited by this background [81].

A new detector (the iZIP) was designed to provide a solution to discriminate the surface event background. The ionization electrodes are interleaved between the phonon sensors, as shown in Fig. 2.9, which allows readout of both ionization and phonon energy on each detector face. Just as importantly, the phonon TES rails are maintained at $0 \mathrm{~V}$ while the ionization electrodes are kept at opposite potentials $( \pm 2 \mathrm{~V}$ in standard operation) on either face. This configuration produces a unique electric field within the crystal (Fig. 2.10) wherein the ionization from surface events will largely be collected on one side of the detector face. The CDMS and EDELWEISS collaborations have shown that the interleaved design allows for robust rejection of surface events. An analysis cut on asymmetric charge collection 

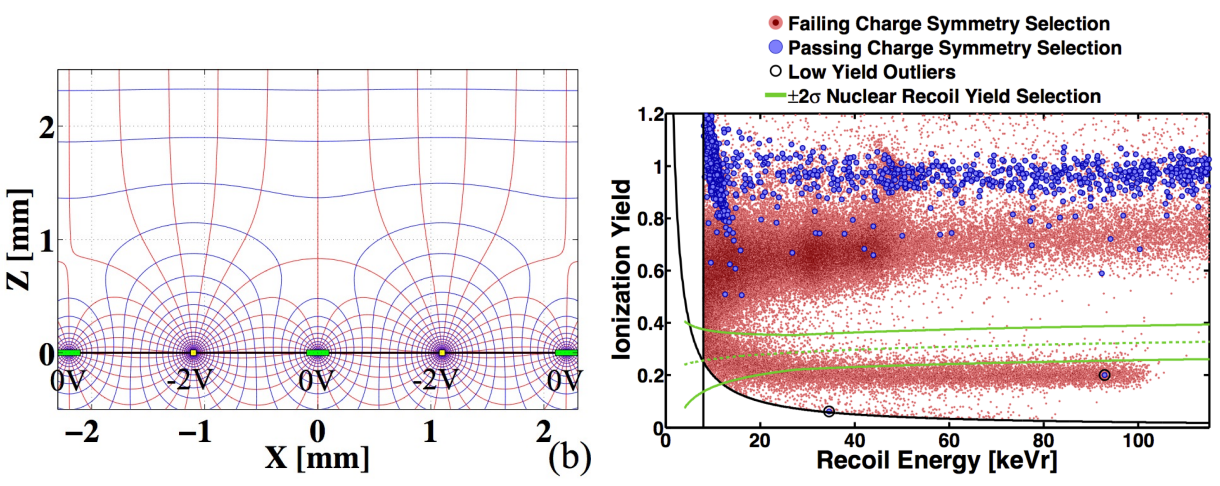

Figure 2.10: (left) The electric field and potential lines produced from the phonon rails (yellow) and charge electrodes (green). Notice that the unique surface E-field extends $\sim 1 \mathrm{~mm}$ into the crystal and therefore surface events within this margin should exhibit asymmetric charge collection. (right) Data from iZIP detector T3Z1 showing surface event discrimination (discussion in main text). Internal CDMS figure, used with permission, from [81].

on side 1 vs. side 2 of the iZIP rejects the surface events.

Figure 2.10 (right) shows the ionization yield vs. recoil energy for events from 900 hours of exposure of a Soudan iZIP detector denoted as T3Z1 (the top detector in tower 3 ) to a ${ }^{210} \mathrm{~Pb}$ source. The ${ }^{210} \mathrm{~Pb}$ source was found, as expected, to produce $\sim 130$ surface electron recoils per hour via beta decay. These events exhibit reduced ionization yield and fail the symmetric charge cut. They populate the region above the $2 \sigma$ nuclear recoil band and below ionization yields of $\sim 1$. The events below the germanium nuclear recoil band but also failing the symmetric charge cut are surface events from recoiling ${ }^{206} \mathrm{~Pb}$ nuclei (the end product of the ${ }^{210} \mathrm{~Pb}$ decay). The colored blue dots are events that pass the symmetric charge and accordingly show large ionization yield corresponding to bulk electron recoils. Out of the 90,000 events in this plot, two outliers exist that pass the charge symmetry cut but show low ionization yield, which are blue and circled in black [81]. Overall, this study demonstrates robust surface rejection capability of the iZIP.

The partitioning of channels on the iZIP, with 3 inner phonon channels and an outer annulus channel as shown in Fig. 2.5, provides $\mathrm{x}, \mathrm{y}$, and $\mathrm{z}$ information about the position of the event in the detector. The prompt phonon signal leads to a larger pulse in the channel closest to the event and a smaller pulse in the channel furthest from the event, and so we can use a linear combination of the optimal filter amplitude from each channel to 
make phonon-based position estimates. We label the calibrated individual channel amplitudes as pXOF, where X refers to channel A,B,C, D, or the sum of the channels "sum". Then with the channel layout as shown in Fig. 2.11, each side of the iZIP (as indicated by the $i$ index) provides an $\mathrm{x}$ position estimate of

$$
\operatorname{pxpartOF}_{i}=\frac{\cos \left(30^{\circ}\right) \cdot \mathrm{pdOF}_{i}+\cos \left(150^{\circ}\right) \cdot \mathrm{pbOF}_{i}+\cos \left(270^{\circ}\right) \cdot \mathrm{pcOF}_{i}}{\mathrm{psumOF}_{i}}
$$

and a y position estimate of

$$
\operatorname{pypartOF}_{i}=\frac{\sin \left(30^{\circ}\right) \cdot \mathrm{pdOF}_{i}+\sin \left(150^{\circ}\right) \cdot \mathrm{pbOF}_{i}+\sin \left(270^{\circ}\right) \cdot \mathrm{pcOF}_{i}}{\mathrm{psumOF}_{i}} .
$$

With the energy partitioned into only 3 channels, the resolution of these partition estimates are never interpreted to be exact, and Fig. 2.11 demonstrates this where the distribution of a set of events uniformly distributed in the detector shows up as a "partition triangle," instead of a circle. Despite the apparent crudeness of these partition estimates, they are useful for checking the individual channel calibrations as well as identifying certain electronic glitch or instrumental events that only cause a signal on one of the channels (an example of this type of event is discussed in Sec. 4.6). A $\mathrm{z}$ position estimate is given by the normalized difference in phonon energy between the two sides:

$$
\text { pzpartOF }=\frac{\text { psum1OF }- \text { psum2OF }}{\text { psum1OF }+ \text { psum2OF }}
$$

The additional fast template information from the two template OF fitting has been used to enhance position information in particular for the development of a radial parameter for single-sided iZIP readout, as discussed in Sec. 4.8.

\subsection{CDMSlite Detectors}

In 2012, subsequent to the development of the iZIP, CDMS explored the operation of an iZIP detector in an alternative configuration in which a higher bias is applied across the detector. This higher detector voltage amplifies the ionization signal by producing NTL phonons. Instead of biasing the detector so that there is a $4 \mathrm{~V}$ potential difference between the faces, roughly $-70 \mathrm{~V}$ is applied. This custom biasing configuration required some modifications 

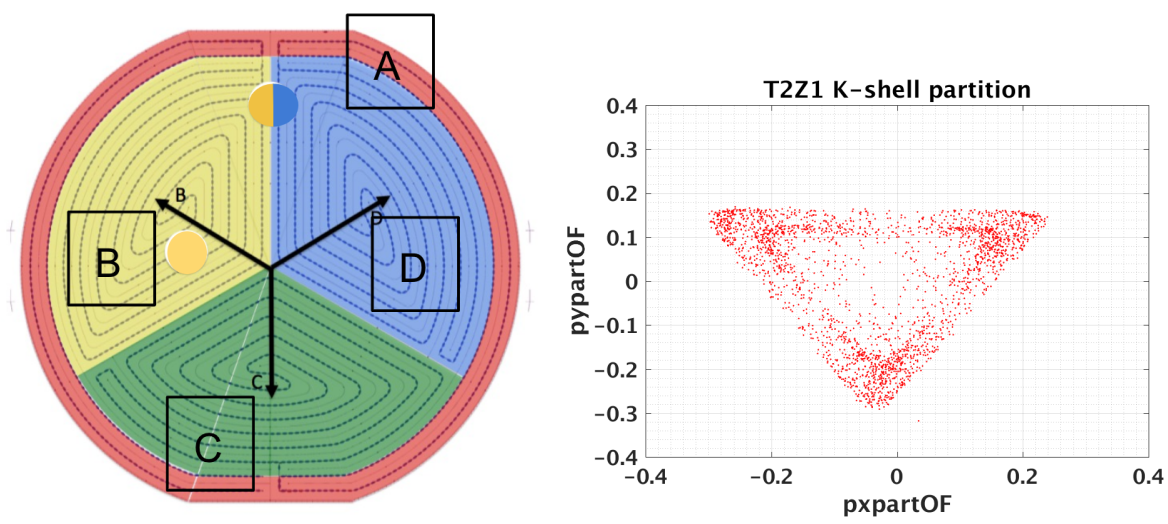

Figure 2.11: (left) The iZIP phonon channel partition on a single side, with channel names labeled. (right) The px-py partition for uniformly distributed events for an iZIP detector, where the triangular shape is due to the channel layout as well as the partition variable definition (Eq. 2.17, 2.19).

to the electronics. A single side of the detector was biased to high voltage, while the other side was grounded, and only the phonon channels and charge channels on the grounded side were read out, as shown in Fig. 2.12.

The primary advantage of the CDMSlite mode is that the energy threshold of the detectors is lowered. The NTL amplification increases the signal, but not the noise, of events so that low energy recoils are able to be measured and reconstructed. Without NTL amplification these events would be "buried" under readout noise. The advantage of a low detector threshold is increased sensitivity to a low mass DM signal - kinematically recoils from lighter DM particles deposit less energy in the detector. Figure 2.13 demonstrates the shifting of the DM spectral shape to lower energy as a function of DM mass and the importance of a low threshold. For example a detector with a $2 \mathrm{keV}$ nuclear recoil threshold has no sensitivity to $2 \mathrm{GeV} / c^{2} \mathrm{DM}$ nuclear recoil signal. The CDMSlite detectors have achieved thresholds of $\sim 0.5 \mathrm{keV}_{\mathrm{nr}}$ and are therefore sensitive to DM masses lower than $2 \mathrm{GeV} / c^{2}$.

The primary disadvantage of the CDMSlite operating mode is that ER/NR discrimination by using the partitioning of the energy between $e^{-} / h^{+}$pairs and phonons is no longer possible. This is the sacrifice that is made for the lower detector threshold: as is clear from Eq. 2.6 and 2.9, a large detector voltage and correspondingly large production of NTL phonons creates a strong correlation between the phonon signal and the $e^{-} / h^{+}$pair signal for both ERs and NRs. The result of this tradeoff between a lower threshold 


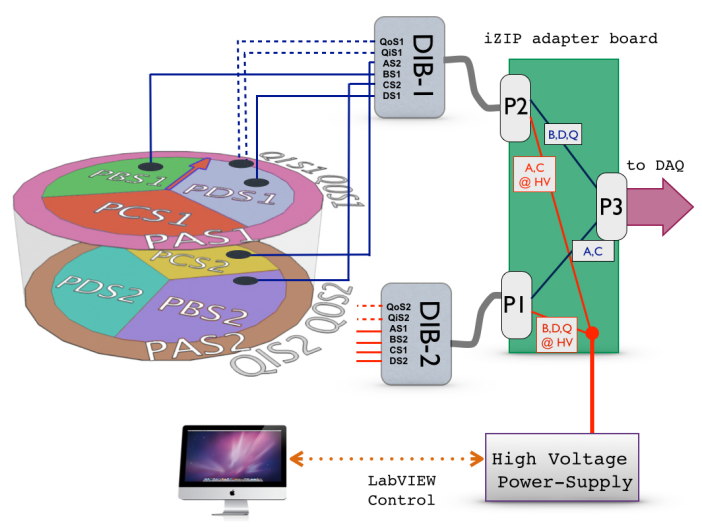

Figure 2.12: A rough schematic of the custom CDMSlite biasing electronics, which shows that only a single side of the detector is read out while the other side is biased using the "High Voltage" power supply (credit: R. Thakur [83]).

and ER/NR discrimination is that the CDMSlite detectors have an "irreducible" source of background from the ER events. The three CDMSlite analyses have therefore been background limited. Each analysis has improved upon the methods of the background reduction and modeling techniques of the earlier runs, and despite the irreducible ER background some CDMSlite analyses have produced world-leading limits for WIMP masses less than $5 \mathrm{GeV} / c^{2}$ at the time of publication [82].

The higher background rate in the CDMSlite detectors from ERs is reduced slightly in the low energy region of interest (ROI) - where the detectors are most sensitive to low-mass DM-because ER background events are amplified by a larger fraction than NR signal events. This is just a consequence of the total phonon energy dependence on the ionization yield, as shown in Eq. 2.9. The effect of the larger ER amplification is to "stretch" ER backgrounds relative to the NR signals, which leads to the reduced ER background rate in the ROI.

\subsubsection{CDMSlite Biasing and Readout Configuration}

The CDMSlite biasing configuration led to a number of non-ideal features of the detector response relative to the iZIP operation. First, with half the channels being read out, the signal to noise ratio was $\sqrt{2}$ worse relative to what it could have been if all channels could be read out (assuming uncorrelated noise between the channels). The single-sided readout also 


\subsection{CDMSlite Detectors}

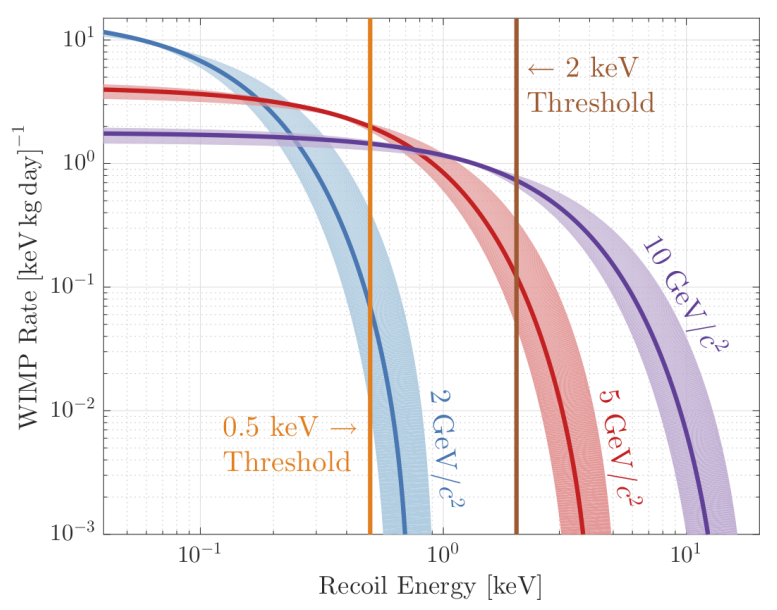

Figure 2.13: DM recoil spectra for different DM masses. The vertical lines show example energy thresholds and demonstrate the importance of threshold for sensitivity to a low mass DM-nuclear recoil scattering. The CDMSlite detectors have achieved thresholds of $\sim 0.5 \mathrm{keV}_{\mathrm{nr}}$. (credit: M. Pepin [84])

required the development of new estimates of the event position, which are described in Sec. 4.8. In addition, the surface events that the iZIP's unique electric field was designed to remove could not be discriminated against with the CDMSlite biasing configuration. We revisit the modeling of this background in the CDMSlite detectors in Chapter 4.

The potentially most significant side effect of the CDMSlite biasing configuration was the non-uniformity of the electric field in the detectors. The non-uniformity arises because the materials surrounding the detector (e.g. the housing) are grounded, and this distorts the electric field. One crosssectional slice of the detector is shown in Fig. 4.39, where near the detector sidewall the $\Delta V$ can be smaller than the applied voltage. Therefore, for events at high radii, the voltage drop experienced by an electron-hole pair (and thus the NTL amplification) can be reduced such that the reconstructed energy is significantly lower than for the same energy events at lower radii. Section 4.8 explains how we model and remove these events when analyzing data from the CDMSlite detector.

\subsubsection{Calibration}

We calibrate the CDMSlite detectors by activating the detector's ${ }^{70} \mathrm{Ge}$ with a ${ }^{252} \mathrm{Cf}$ neutron source. Activation of ${ }^{70} \mathrm{Ge}$ by neutron capture produces 


\begin{tabular}{c|c|c|} 
Shell & Energy [keV] & Probability \% \\
\hline K & 10.37 & 87.6 \\
L & 1.30 & 10.5 \\
M & 0.160 & 1.78 \\
N & 0.0015 & 0.14
\end{tabular}

Table 2.5: The energies and probabilities of electron capture for ${ }^{71} \mathrm{Ge}$. The $N$-shell is included, though events from this EC have never been observed in the CDMSlite detectors because of the low energy of this process.

${ }^{71} \mathrm{Ge}$, which decays by electron capture with a 11.43 day half-life [85]. This process is given by:

$$
\begin{aligned}
{ }^{70} \mathrm{Ge}+n & \rightarrow{ }^{71} \mathrm{Ge} \\
{ }^{71} \mathrm{Ge}+e & \rightarrow{ }^{71} \mathrm{Ga}+\nu_{e} \\
{ }^{71} \mathrm{Ga} & \rightarrow{ }^{71} \mathrm{Ga}+\gamma \text { 's }+e{ }^{\prime} \mathrm{s} .
\end{aligned}
$$

The $\gamma$ 's $+e$ 's represent the decay products from the electron recoil as ${ }^{71} \mathrm{Ga}$ reorders its electron shell into a stable state [67]. These decays produce peaks at the $K$-, $L$-, and $M$-shell binding energies of ${ }^{71} \mathrm{Ga}$ of $10.37,1.30$, and $0.16 \mathrm{keV}$, respectively [86]. The energy spectrum with $K$-, and $L$-, shell ${ }^{71} \mathrm{Ge}$ electron capture peaks is shown in Fig. 2.14. The most prominent peak is the $K$-shell ( $1 s$ orbital) peak. Decays from the lower energy peaks $(L$ - and $M$ shell peaks) occur at a lower rate because of smaller electron wavefunctionnucleus overlap as well as electron binding energy factors. Formulae for computing electron capture probabilities are computed in Ref. [87, 88], and tabulated in Table 2.5. In the data we observe a ratio between $K$ - and $L$ shell peaks that is consistent with the expected relative rates.

The $K$-shell peak is used to calibrate the energy scale and correct for any time and position variation in the detector response. The $L$ - and $M$ shell peaks are used to check the resulting energy scale for linearity. These relatively low energy ${ }^{71} \mathrm{Ge}$ activation peaks are critical for the energy scale calibration of CDMSlite because, unlike the calibration lines from other common calibration sources $\left({ }^{133} \mathrm{Ba}\right.$ and $\left.{ }^{252} \mathrm{Cf}\right)$, these events have sufficiently low energy that they do not saturate the TESs in CDMSlite operation.

\subsubsection{CDMSlite History: Run 1 and Run 2}

The early commissioning work for CDMSlite surveyed the array of SuperCDMS iZIP detectors (the array pictured in Fig. 4.4) to determine which 


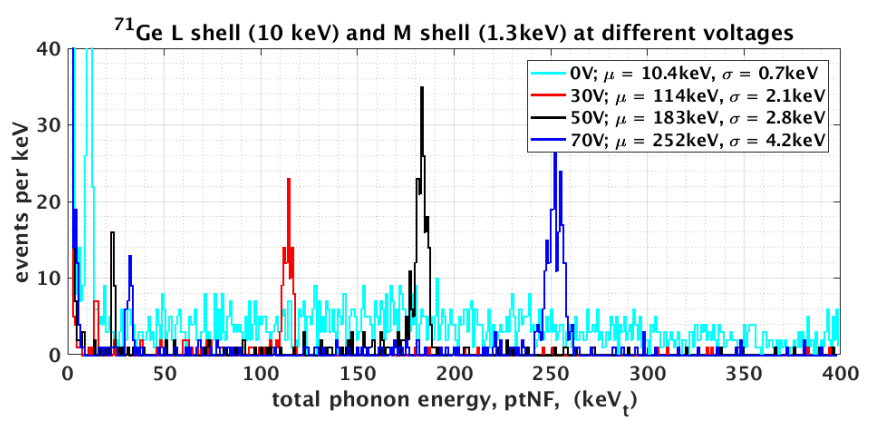

Figure 2.14: The reconstructed total phonon energy of data with ${ }^{71} \mathrm{Ge}$ electron capture peaks present. The colors label data taken at different detector voltages, where the NTL amplification is apparent at the $30 \mathrm{~V}, 50 \mathrm{~V}$, and $70 \mathrm{~V}$ data. The energy scale of the data at each voltage is calibrated so that the $K$-shell peaks appear at the correct energy (i.e. the total phonon energy given by Eq. 2.9), and additional details and insights gained from these "voltage scan" data are discussed in Sec. A.1.

detector was best suited for CDMSlite operation. In this selection process, one of the primary factors was the detector's leakage current, which imposed a voltage maximum below which the detector exhibited stable operation. While the dominant mechanism of charge leakage in the iZIP detectors is unknown (and is likely from a combination of mechanisms and variable between detectors), the basic idea is simple. When the voltage applied across the detector is sufficiently high, a small current will start to flow across the detector (i.e. the detector resistance is not infinite). This current could come either from the interface of the detector or from charges in shallow-potential impurity sites in the bulk of the detector (both of which are depicted in Fig. 2.15), or from another source. The leakage charges will produce NTL phonons that will be read out by the TES phonon amplifiers, and because the leakage current is a quasi-Poissonian process, the leakage current creates a source of noise in the phonon signal.

To first order, the leakage current in the tested iZIP detectors at Soudan was found to be negligible until a certain "breakdown" voltage was reached. This "breakdown" effect was also observed by P.N. Luke in one of the first publications describing the NTL effect [76], as depicted in Fig. 2.15 (center). The optimal operating voltage before breakdown in the iZIP detectors was found to be $\sim 70 \mathrm{~V}$. At these voltages, even relatively large detector resistances, $\sim 1 P \Omega$, will produce leakage currents of $4 \times 10^{5}$ electrons/sec 
through the detector, which is a significant source of NTL phonon noise in the phonon amplifier. Leakage current studies are discussed in more detail in Sec. 3.2, and efforts to improve the detector leakage currents for future CDMS detector are underway.

CDMSlite Run 1 operated the detector in the middle of the fifth tower, as depicted in Fig. 4.4 and referred to as T5Z2, that had low leakage currents. The optimal voltage was found to be $69 \mathrm{~V}$, where above this voltage the increase of a leakage current led to elevated noise. The detector was operated for a relatively small period of time in 2012 and the DM search live time totalled $6.25 \mathrm{~kg}$-days. The analysis achieved an analysis threshold of $170 \mathrm{keV}_{\text {ee}}$, and the limit placed on DM-nucleon cross section was world-leading below $6 \mathrm{GeV} / c^{2}$ DM mass at the time of publication[82].

CDMSlite Run 2 also operated the T5Z2 detector, but did so for significantly longer (70 kg-days over the course of 2014) and biased the detector to $70 \mathrm{~V}$. A number of operational and analysis improvements were made in this run. The three most significant operational improvements are described in more detail below.

1. Pre-biasing. Run 1 observed that the noise in the minutes following the HV biasing of a detector was higher relative to later times. At the time the hypothesis for this excess noise was a higher leakage current from trapped charge lying in shallow potentials, and the charges were released once the detector was biased to high voltage; this hypothesis is made in Appendix B of Ref. [83]. The period of higher noise was observed even following the standard CDMS detector neutralization procedure, which consists of exposing the detector to $1.31 \mathrm{eV}$ LED photons prior to operation. The motivation behind this neutralization is to nullify any possible trapped charge, as described in Appendix $\mathrm{H}$ of Ref. [89]. Unfortunately, there was no evidence that the neutralization procedure eliminated the period of elevated noise following HV biasing. It was found that this period of elevated noise could be shortened if the detector voltage was raised above the operating voltage for $\sim 10$ minutes, with the hypothesis that the higher voltage emptied the traps more efficiently. The noise at later times, after the pre-bias period, was also found to be lower than without the pre-bias. Fig. 2.15 bottom shows the baseline RMS baseline noise with and without the pre-bias, with the triangles (without pre-bias) showing a worse resolution than the circles (with pre-bias).

During the second CDMSlite run, a 80V prebias was used for 10 minutes prior to the data taking with the detector biased at the $70 \mathrm{~V}$ 

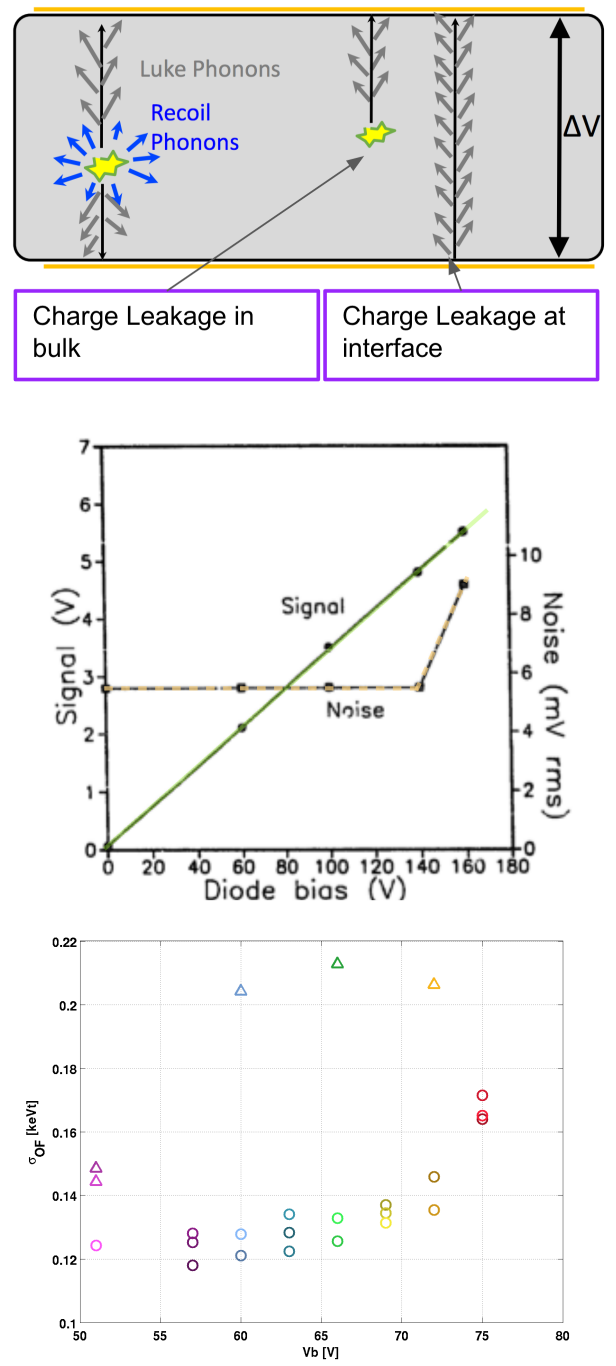

Figure 2.15: (top) A simple depiction of a regular event from a particle interaction alongside two leakage events, one from the detector bulk and one at the detector interface. (middle) From P. Luke's publication, showing the signal-to-noise benefits of NTL amplification up to $\sim 140 \mathrm{~V}$ for the diode detector described in Ref. [76]. (bottom) The baseline total phonon noise RMS, labeled as " $\sigma_{O F}\left[\mathrm{keV}_{\mathrm{t}}\right]$ " for the T5Z2 detector, showing an increase in the noise above $\sim 60 \mathrm{~V}$ and a severe worsening of the noise above $\sim 70 \mathrm{~V}$. The triangles on this plot provide supplemental measurements of the noise RMS without pre-biasing, demonstrating the effectiveness of the pre-bias procedure. 
operating voltage ${ }^{6}$.

2. Accelerometer Vibration Readings. Analysis of the phonon signal power spectral density (PSD) (e.g. Fig. 4.9) on Soudan detectors showed a broadband increase of noise at low frequencies, in contrast with what is expected from fundamental TES noise, which does not increase at low frequencies [78]. A subsequent series of studies at Soudan was used to trace this LFN excess back to vibrational sources in the Soudan setup [90]. Accelerometers were installed on the cryocooler, known to be the most vibrationally active part of the experiment, and event rates and LFN noise events were indeed found to be strongly correlated with the periods of higher amplitude cryocooler vibrations. In Run 2, the accelerometer readings were used to identify and isolate periods of particularly high LFN. Then, particularly restrictive cuts to discriminate against LFN induced events were used for those periods with high noise. The accelerometer readings are discussed in the context of CDMSlite Run 3 in Sec. 4.3.2.

3. High Voltage Current Readings. The schematic diagram shown in Fig. 2.16 depicts the HV biasing scheme of CDMSlite detectors, where the schematic is simplified to not show any of the readout electronics. A critical part of the biasing scheme, which was added prior to Run 2 , is to continuously monitor the HV current $\left(I_{H V}\right)$ in order to determine the detector voltage relative to the applied voltage. With an effectively infinite detector resistance, the nominal $I_{H V}$ would be 0 amps. However, parasitic resistance to ground (shown here as $\mathrm{R}_{p}>$ $1 \mathrm{G} \Omega$ ), enables a small current (up to $120 \mathrm{nA}$ ) to be sourced by the HV power supply. While the exact location of this parasitic resistance (i.e. current leak) is unknown, there is strong evidence that the leak occurs at a component of the room temperature electronics, potentially on the CDMSlite HV biasing board.

A large bias resistor $\left(\mathrm{R}_{b}=196 \mathrm{M} \Omega\right)$ is used to protect against significant current flow into the cryostat in the case of a short, however that large bias resistor also results in a significant decrease of the voltage at the detector, even for small HV leakage currents. For example, a $120 \mathrm{nA}$ leakage current results in a reduction of $24 \mathrm{~V}$ at the detector. By reading the HV power supply current over the course of Run 2, the voltage of the detector was calculated on an event-by-event basis.

\footnotetext{
${ }^{6}$ This same pre-bias procedure was used for CDMSlite Run 3, where a $85 \mathrm{~V}$ was used as the pre-bias point for the nominal $75 \mathrm{~V}$ operating voltage, as described further in Sec. 4.
} 


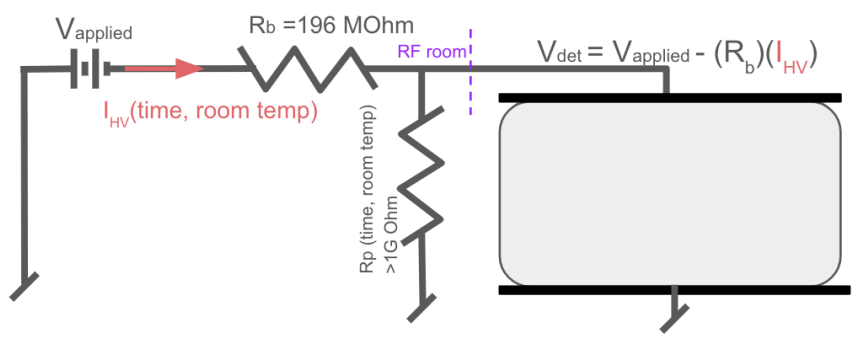

Figure 2.16: A simple schematic of the CDMSlite biasing circuit.

The largest current measured in Run 2 was found to be $25 \mathrm{nA}$, which corresponds to a $6.5 \%$ difference between the applied voltage and the detector voltage, which was corrected for in the analysis. ${ }^{7}$

In addition to the operational improvements made for CDMSlite Run 2, numerous advances were also made during the analysis and described in M. Pepin's thesis [67].

One significant improvement was the development of a parameter that identified events that occurred at high detector radii that would experience reduced NTL amplification and therefore would be misreconstructed to a lower energy [91]. Further improvements to the radial fiducial volume modeling were made for the CDMSlite Run 3 analysis, and we save the more detailed description of the radial parameter to that section of this thesis (Sec. 4.8).

The CDMSlite Run 2 analysis also found environmental variables (e.g. cryostat base temperature) and pulse-shape characteristics (e.g. pulse peakiness) that were correlated with the energy estimate of an event, and the analysis corrected the energy scale with these variables and significantly improved the resolution of the ${ }^{252} \mathrm{Cf}$ calibration peaks. Because the Run 3 analysis employs similar energy corrections, we describe the details of the procedure in Sec. 4.4.1 in the context of the Run 3 data.

\footnotetext{
${ }^{7}$ In contrast to Run 2, the largest current measured in Run 3 was $120 \mathrm{nA}$, and the current was significantly less stable throughout the run. The incorporation of the HV current readings for the Run 3 analysis is discussed in Sec. 4.3.3.
} 


\section{CDMSlite Run 1 and Run 2 DM Exclusion Limits: The Optimum Interval Method}

The operational and analysis improvement described above led to the Run 2 analysis achieving an analysis threshold of $0.056 \mathrm{keV}_{\mathrm{ee}}$. The addition of the radial cut resulted in a final energy spectrum of events that was largely understood, where different populations of events could be attributed to known sources. The final Run 2 energy spectrum (converted to the nuclear recoil energy) is shown in Fig. 2.17, where the two background peaks are from $M$ - and $L$-shell events. Despite an understanding of most background sources, at the lowest energies $\left(0-0.75 \mathrm{keV}_{\mathrm{nr}}\right.$ in Fig. 2.17) the ability to distinguish a good event (caused by an energy deposition in the detector) from an instrumental event (caused by a noise fluctuation that was large enough to generate a trigger and read out the "event") is diminished. The Run 2 analysis cuts were defined in such a way that there was a significant uncertainty about whether the events at the lowest energy were good events or instrumental events.

Because of the potential leakage of instrumental events past the Run 2 analysis cuts, the analysis used a conservative technique for setting a DM exclusion limit. The technique is called the Optimal Interval (OI) method developed by S. Yellin [92, 93], and is succinctly described in the title of his 2002 paper: "Finding an Upper Limit in the Presence of Unknown Background." The method does not require a background model, which significantly simplifies the analysis. This is because, not only do background models not need to be developed, but also instrumental events (for which there is no physically motivated background model) can leak past the cuts without serious consequences for the analysis.

The OI method works by interpreting all events as potential signal events and then computing a signal size that is incompatible with the data at the $90 \%$ confidence level (CL). The method to quantify "incompatible" uses the expected signal shape and the energy intervals between events. An example interval in the CDMSlite Run 2 data is shown in Fig. 2.17. For the DM signal shapes shown in Fig. 2.13, one can find an interval where the background is low and where the signal shape is especially high that will be able to optimally exclude the signal at some magnitude at $90 \% \mathrm{CL}$; this interval is the "optimal interval." The OI method finds this interval and applies an appropriate trials factor for selecting an interval that gives the best limit (i.e. it accounts for the "look elsewhere" effect).

This approach has a number advantages in that it does not require a background model and is conservative, but one clear disadvantage is that 


\subsection{CDMSlite Detectors}

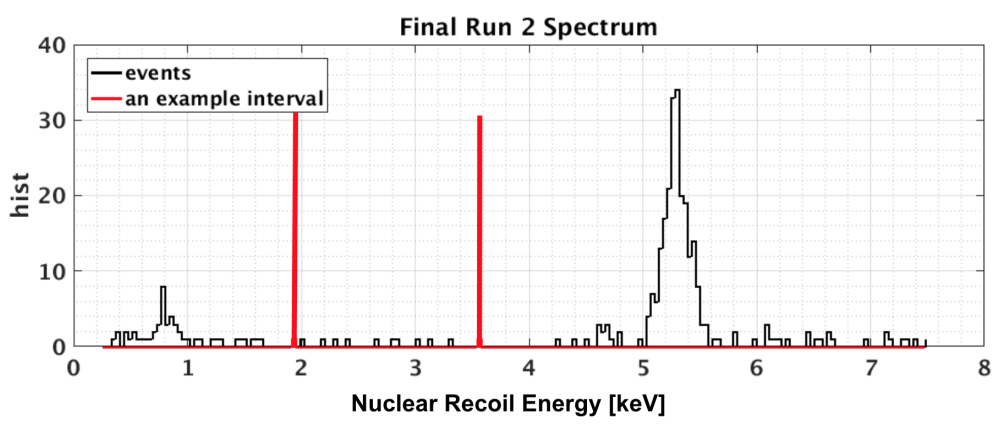

Figure 2.17: The CDMSlite Run 2 energy spectrum after all cuts have been applied, with the energy of the events measured by the non-stationary optimal filter, and converted to the nuclear recoil energy using a Lindhard $k$ parameter of 0.159 . One of the energy intervals is highlighted between events as a visualization of the energy intervals considered by the optimum interval method. The optimal interval considers intervals between all pairs of events (not necessarily adjacent pairs of events)

it cannot be used to discover a signal in the data; it can only be used to set upper limits. The Run 1 and Run 2 analyses used this method and therefore were not discovery-potential analyses, and in the Run 3 analysis we explicitly address this shortcoming and employ a likelihood approach to search for a signal in the dataset with discovery potential. Despite this shortcoming of the OI method, at the time of publication, the Run 2 analysis excluded new spin independent WIMP-nucleon parameter space between 1.6 and $5.5 \mathrm{GeV} / c^{2}$. Both the Run 1 and Run 2 90\% CL upper limits are shown in Fig. 2.18. 


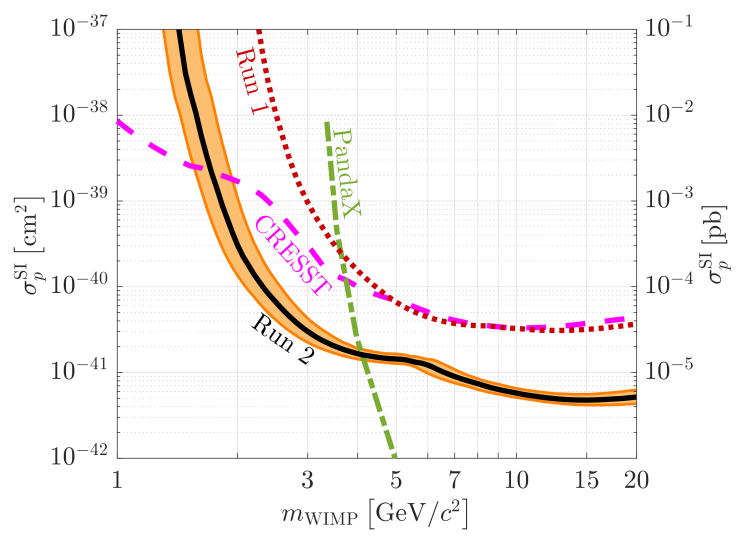

Figure 2.18: Spin-Independent WIMP-nucleon cross section 90\% CL upper limits from Run 1 and Run 2 (black solid with 95\% uncertainty band) compared to the other most sensitive results in this mass range (at the time of publication of the Run 2 result): CRESST [94] below $1.6 \mathrm{GeV} / c^{2}$ and PandaX-II [43] above $4 \mathrm{GeV} / c^{2}$. 


\section{Chapter 3}

\section{Detector Characterization}

\subsection{Detector Resolution}

The CDMS detectors' energy resolution is one of the primary drivers of their sensitivity to low-mass DM. This is because, as previously discussed in Sec. 2.5 and depicted in Fig. 2.13, the expected signal from DM particles of increasingly lower mass is higher at low energy. Better energy resolutions allow the detectors to resolve lower energy recoils, effectively increasing the detector's exposure to a low-mass DM signal. In this chapter we focus only on the resolution of the phonon amplifiers, as opposed to the $e^{-} / h^{+}$ amplifiers, because (1) the phonon amplifiers are intrinsically more sensitive and therefore are the primary drivers of the sensitivity, and (2) the capacity to improve the phonon amplifiers with new detector designs is greater and therefore many CDMS R\&D efforts are channeled in this direction.

Detector resolutions are typically thought of as varying as a function of energy (e.g. see Sec. 5.1.1), but in this chapter we focus on the "baseline" resolution because it is particularly important for the detector's sensitivity to the lowest energy recoils. The baseline resolution is a measurement of the detector's resolution at zero energy. Therefore, a lower baseline resolution corresponds directly to a lower detector threshold and the ability of the detector to observe lower energy recoils above the detector noise.

Detector research and development efforts seek to improve the baseline resolution by either increasing the amplifier signal or decreasing the amplifier noise. Efforts to increase the signal include NTL amplification (discussed in Sec. 3.2) as well as increasing the phonon collection efficiency of the amplifiers (measured in Sec. 3.1.4). Efforts to measure, understand, and reduce the phonon noise are discussed in Sec. 3.1.2 and Sec. 3.2.

We use two complementary methods of calculating the baseline resolution. The simpler method is a direct measurement using a combination of calibration data and noise data acquired from the detector. The more involved method combines characterizations of the detector's electronic and phonon collection properties with resolution models from signal processing and optimal filter theory [78]. The more detailed analysis of the baseline 
resolution indicates that the signal-to-noise ratio should be considered as a function of frequency, which has implications for the preferred pulse shape, or pulse bandwidth, as discussed in Sec. 3.1.5.

Below we compare the results from these two different detector baseline resolution calculations for a number of prototype detectors fabricated as part of the R\&D effort for SuperCDMS SNOLAB. These detectors were fabricated with lower TES transition temperatures $\left(T_{c}\right)$ than the Soudan iZIP detectors, which was one of the primary design changes to reduce the phonon noise. To first order, this noise reduction occurs because the projected dominant noise source for SNOLAB, the thermal fluctuation noise between the TES and the cooling bath, is reduced at lower temperatures; for further discussion see Ref. [95]. The detector resolution measurement results demonstrate improved detector performance of the prototype detectors relative to the SuperCDMS Soudan detectors, and the achievement of detector performance goals for the SuperCDMS SNOLAB experiment.

\subsubsection{Data-Driven Resolution Measurement}

The direct measurement of a detector's baseline resolution requires calibration data and "noise" data. The calibration data is used to determine a calibration factor to convert the output of the phonon amplifier (in amps) to an energy (in eV). The use of the "noise" data is less obvious; it consists of time series data that are digitized and read out in the same way as standard events, except that the data acquisition system is triggered randomly with the intention of reading out a time series in which no event occurs. Therefore, noise data are frequently referred to as "random triggers" or simply as "randoms."

The random triggers are fit by the $1 \mathrm{D}$ optimal filter (OF) algorithm and the amplitude of the fit is recorded. As shown in Fig. 3.1 (left), even though there has been no energy deposition in the detector when the random trigger was digitized, the OF algorithm will fit a small non-zero amplitude because of the noise fluctuations. The noise fluctuation in this example has caused the amplitude to fit to a negative value (which is just as likely as a positive value when no signal exists in the data). It is clear that the OF amplitude fit of the random triggers can be used to characterize the detector noise because larger noise fluctuations will result in a larger span of the fit amplitudes to both positive and negative values. The standard deviation of the distribution of OF amplitude fits to a collection of random triggers gives the baseline resolution. 

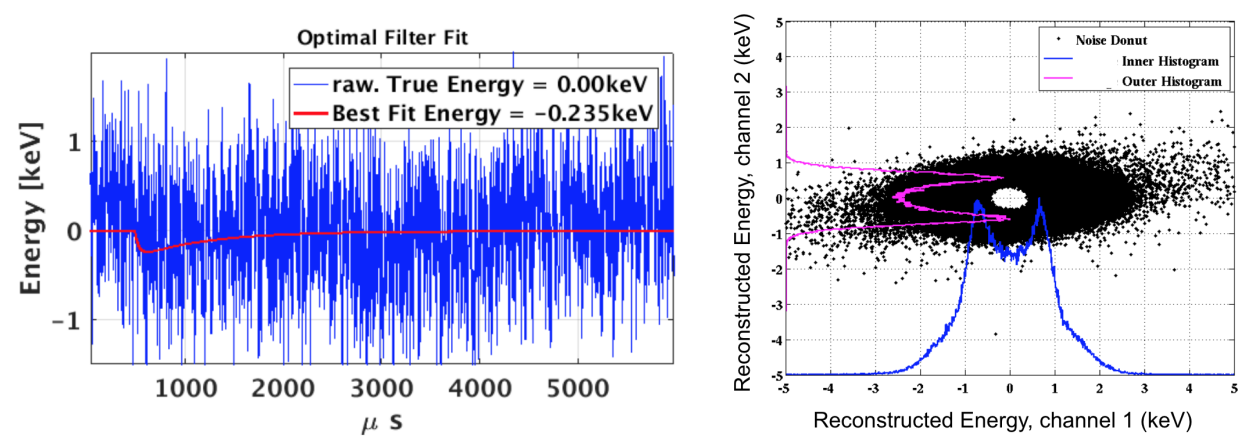

Figure 3.1: (left) Example simulated random trigger (noise only) with the best fit OF template overlaid in red. Even though there is zero signal, noise fluctuations result in the $\mathrm{OF}$ algorithm fitting a small non-zero amplitude. (right) The $\mathrm{OF}$ amplitude fit results (from a detector with two readout channels) of many random triggers, when the OF algorithm has no constraint on the time delay search window [96].

\section{Constraining the Optimal Filter Time Offset}

Figure 3.1 (right) shows the histogram of many OF fit amplitudes to random triggers (for a two-channel detector), and also reveals a subtlety in the $\mathrm{OF}$ fitting of random triggers. The bimodal distribution occurs because of the time-offset degree of freedom (which minimizes the OF $\chi^{2}$ for left-right shifts of the time domain template as described in Sec. 2.3), which allows the OF fit to find the largest noise fluctuation that resembles a signal pulse within the entire time series of data. The OF then returns the amplitude of that fluctuation. Because it is likely that the OF finds a noise fluctuation that is significantly different than zero, the distribution shown in Fig. 3.1 (right) has a deficit of events at reconstructed amplitudes at and around $0 \mathrm{keV}$.

This undesired time-offset degree of freedom of the standard OF is disabled when measuring the detector baseline resolution: the time offset $\left(t_{0}\right.$ in Eq. 2.16) is clamped to $t_{0}=0$ in the fit. With this adjustment, we refer to the OF amplitude of the total phonon trace as "ptOF0," where the 0 indicates that the $t_{0}$ has been forced to 0 , and with this adjustment OF fits to random triggers result in a Gaussian distribution, as shown for example in Fig. 3.2, and the $1 \sigma$ width of this Gaussian distribution defines the baseline resolution. 


\section{Efficient Calibration of low- $T_{c}$ Detectors}

The lower $T_{c}$ TES transition temperature has the primary advantage of reducing the TES noise, but one negative side effect of the lower transition temperatures is that the TESs exhibit a smaller "dynamic range." That is, low- $T_{c}$ TESs begin to saturate for lower levels of incident power, and this became problematic in the testing of these prototype detectors because the TES began to saturate at the energies used to calibrate the detector. In particular, the $356 \mathrm{keV} \gamma$ events from ${ }^{133} \mathrm{Ba}$ served as an efficient calibration source for the SuperCDMS Soudan iZIP detectors, which were fabricated with $T_{c}$ in the $70-110 \mathrm{mK}$ range. In this section, we show results from a prototype detector (referred to as detector S12C, with the same dimensions and channel layout as the iZIP detectors) with $T_{c}$ in the $60-68 \mathrm{mK}$ range, and the TESs were found to begin to saturate for the $356 \mathrm{keV} \gamma$ events. The efficient nature of the ${ }^{133} \mathrm{Ba} 356 \mathrm{keV} \gamma$ calibration still made it appealing for detector calibration for the resolution studies presented in this section, but because the TES saturation introduced non-linearity into the TES response (and therefore non-linearity into the energy scale of the OF amplitude energy estimate) extra calibration steps were required.

The OF underestimates the energy of saturated events, and so the naïve calibration approach of centering the $356 \mathrm{keV}$ events at that energy results in an energy scale that is biased to low energy. The pulse "integral" energy estimate, which has worse energy resolution than the OF energy estimate but is less prone to energy scale non-linearities from saturation effects than the OF energy estimate, was used to correct the OF amplitude energy estimate. The correction assumed that (1) the integral and OF energy estimates were both linear at low energies and that (2) the integral energy estimate was linear up to $356 \mathrm{keV}$. The latter of these assumptions comes from the fact that the OF estimate is highly sensitive to the pulse shape, whereas the integral is less sensitive. The saturation correction technique centers the $356 \mathrm{keV}$ events at that energy in the integral energy estimate, and also forces a one-to-one relationship between the integral and OF at lower energy. This ensures that at low energy, at the energy where the baseline resolution is measured, the OF energy scale is unbiased. Figure 3.2 left depicts this calibration and correction technique.

With the OF energy scale calibrated, the baseline resolution was determined in units of $\mathrm{eV}$ as shown in Fig. 3.2 right. At the time of measurement, the $52.4 \mathrm{eV}$ resolution measured on $\mathrm{S} 12 \mathrm{C}$ was one of the lowest measured resolutions on an iZIP-style detector. This improvement was one important piece of evidence of the detector noise improvements from lowering the $T_{c}$ 


\subsection{Detector Resolution}
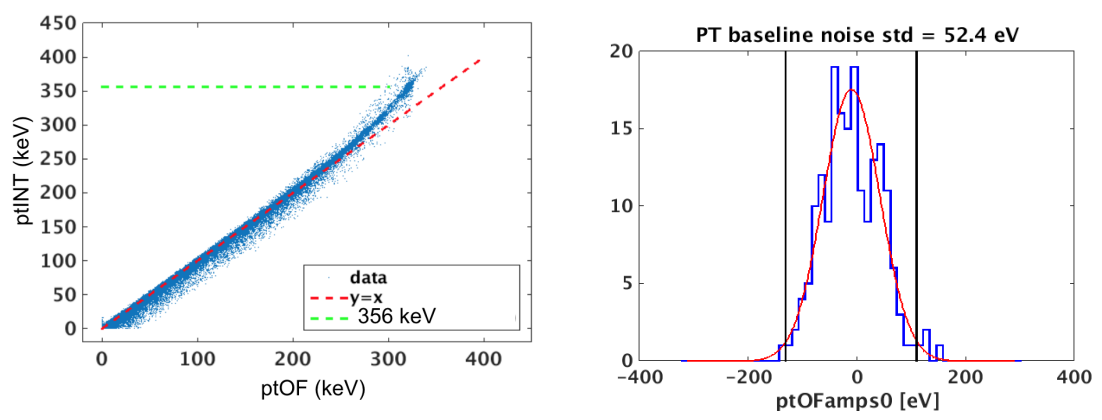

Figure 3.2: (left) Data from ${ }^{133} \mathrm{Ba}$ calibration of the $\mathrm{S} 12 \mathrm{C}$ detector, in the total phonon integral vs. total phonon OF energy estimates. (right) The S12C detector baseline resolution measurement using random triggers and measured using the constrained time-offset $\mathrm{OF}$, giving a resolution of $52.4 \mathrm{eV}$.

of the TES sensors, and the following sections $(3.1 .2,3.1 .3,3.1 .4)$ focus on the TES characterization to improve the understanding of such an energy resolution.

\subsubsection{Phonon Amplifier Noise}

The noise power spectral density (PSD) is the variance of noise as a function of frequency, and is calculated using a collection of random triggers that are uncontaminated by events and are representative of the detector noise. The discrete Fourier transform (DFT) $\tilde{g}_{n}$ of a time series $g_{k}$ is given by:

$$
\tilde{g}_{n}=\frac{1}{N} \sum_{k=-\frac{N}{2}}^{\frac{N}{2}-1} g_{k} e^{-2 \pi i f_{n} t_{k}}
$$

where $N$ is the number of samples in the time series and where $\tilde{g}_{n}$ is evaluated for $n=-\frac{N}{2} \rightarrow \frac{N}{2}-1$ in integer steps. The frequency spacing is given by $f_{n}=n \frac{1}{T}$ where $T$ is the time length of the time series in seconds. The time steps are given by $t_{k}=k \Delta t=k \frac{T}{N}$ and in this notation are symmetrical around 0 . To calculate the PSD $J$, the squared magnitude of the DFT $\left|\tilde{g}_{n}\right|^{2}$ is calculated for each random, and the average value of $\left|\tilde{g}_{n}\right|^{2}$ is calculated for each frequency index $n$ :

$$
J_{n}=2 T \times\left\langle\left|\tilde{g}_{n}\right|^{2}\right\rangle .
$$


The normalization convention for PSDs is notoriously inconsistent, in part because at times it is convenient to consider the positive and negative frequencies over which the Fourier transform is defined and at times it is convenient to consider just the positive frequencies. CDMS has historically considered positive frequencies, and then "folded over" the PSD across the $\mathrm{y}$ axis and applied the factor of 2 .

When considering the TES noise, $g_{k}$ is typically a current in amps, and therefore the units of the PSD, $J_{n}$, is amps ${ }^{2} / \mathrm{Hz}$. Very commonly, instead of considering $J_{n}$ as a representation of the variance of the noise as a function of frequency, we plot the standard deviation, or $\sqrt{J_{n}}$. There are benefits of both units, and $\sqrt{J_{n}}$ is convenient since this quantity is linearly proportional to the baseline resolution of the detector (Eq. 3.16) as discussed in Sec. 3.1.5.

We show multiple PSDs, in units of amps $/ \sqrt{\mathrm{Hz}}$, for the iZIP S12C detector in Fig. 3.3 top. The individual channels are shown by the colored lines, while the total phonon (i.e. traces summed in the time domain) PSD is shown by the solid black line. The quadrature sum of the individual channels is shown by the dashed black line, and it is useful because it represents the total phonon noise that would exist if that noise between the individual channels was uncorrelated. Since fundamental TES noise is uncorrelated between channels and environmental noise sources are more likely to be correlated between channels, comparison of the total phonon and quadrature sum PSDs is useful for diagnosing reducible environmental noise sources. Also, as shown in Sec. 3.1.5, the quadrature noise is useful for deriving a "best case" baseline energy resolution that is achievable if the environmental noise sources are removed.

\subsubsection{TES Voltage Biasing}

The TES is a very sensitive power to current amplifier, and in this section we discuss how to measure the dynamic current response $(\mathrm{d} I)$ of the TES to a change of input power $(\mathrm{d} P)$. The voltage-biased TES schematic is shown in Fig. 3.4 (left) where the shunt resistor $R_{s}$ is significantly smaller than the equilibrium TES resistance, an inductor $L$ is included to facilitate readout of the current through the circuit with a SQUID amplifier (a highly sensitive current to voltage converter), and there is stray parasitic resistance $R_{p}$ in the circuit. It is critical that the TES be voltage-biased (i.e. $R_{s} \ll$ $\left.R_{p}+R_{T E S}\right)$ as opposed to current-biased for stable operation. To first order, this stability condition can be explained when considering the power 


\subsection{Detector Resolution}
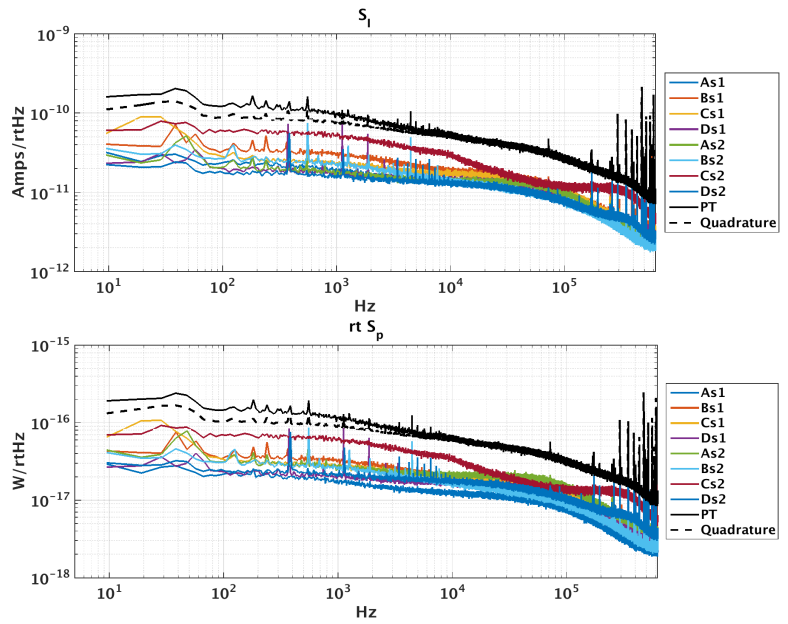

Figure 3.3: (top) The current noise (amps $/ \sqrt{\mathrm{Hz}}$ ) for each channel , the total phonon channel sum, and the quadrature channel sum for the S12C iZIP detector. (bottom) The S12C power noise (watts $/ \sqrt{\mathrm{Hz}}$ ), converted from the measured current noise using the $(\mathrm{d} I / \mathrm{d} P)$ conversion described in Sec. 3.1.4.

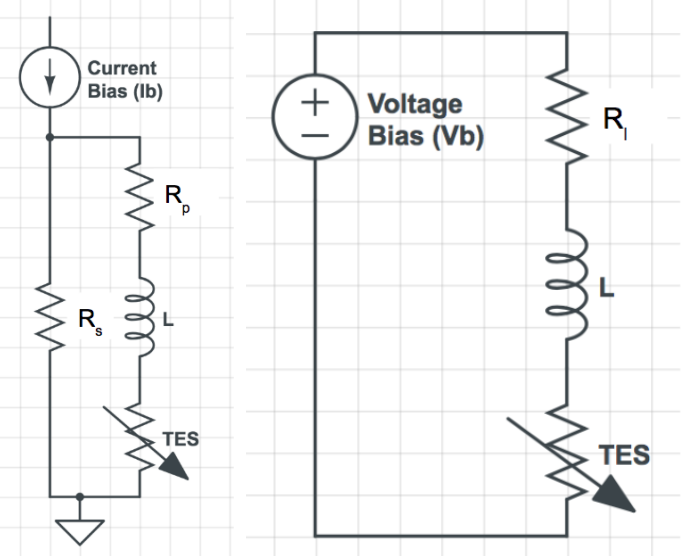

Figure 3.4: (left) Simplified circuit diagram for the TES amplifier, with the shunt resistor $\left(R_{s}\right)$, parasitic resistor $\left(R_{p}\right)$, inductor $(L)$, and TES element labeled. (right) The Thevenin equivalent voltage-biased TES circuit, where $V_{b}=I_{b} \cdot R_{s}$ and the load resistor is given by $R_{l}=R_{p}+R_{s}$. 
dissipated in the TES:

$$
P_{\text {Joule }}=\frac{V^{2}}{R}=I^{2} \cdot R .
$$

When the TES resistance increases upon some incident phonon power from an event in the detector, with a constant voltage across the TES the Joule power dissipated decreases as indicated by Eq. 3.3. This reduction of Joule power for an increase in TES resistance returns the TES to equilibrium and is referred to as negative electrothermal feedback. By contrast for a currentbiased TES, increasing the resistance increases the Joule power dissipated in the TES, thereby further increasing the resistance and this positive feedback loop results in unstable operation. A significantly more thorough consideration of stable voltage-biased TES operation is discussed in Ref. [95] and Ref. [78].

\subsubsection{Phonon Collection Efficiency}

The phonon collection efficiency $\left(\epsilon_{\text {phonon collection }}\right)$ is the fraction of energy from an event that ends up as dissipated power in the TES. It is an important quantity to measure because the baseline energy resolution is directly

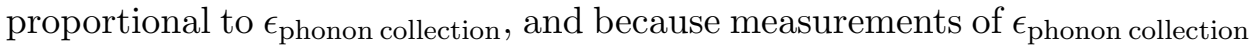
help inform design choices for future detectors. We calculate the energy collected in the TES using the current read out by the TES in combination with measurements of the electronic components of the TES circuit. Then we take the ratio of this collected energy with the known true energy of the event, and define this as the TES collection efficiency:

$$
\epsilon_{\text {phonon collection }}=\frac{E_{\text {collected }}}{E_{\text {true }}} .
$$

Measuring the TES collection efficiency is not only a critical step in calculating the theoretical baseline resolution of the detector (Sec. 3.1.5) as well as the detector leakage current (Sec. 3.2), but it is important to measure for each prototype detector to determine how changes in the detector fabrication process are affecting the TES collection properties. For the prototype detector $\mathrm{S} 12 \mathrm{C}$, we do the phonon collection measurement on a channel-by-channel basis as well as for the full detector (the channel sum).

When the TES is operating in the stable voltage-biased regime, the temperature of the TES never increases or decreases appreciably and rather a power flux $\delta P$ incident on the sensor results in a reduction of current $\delta I$ through the sensor that satisfies $P_{\mathrm{TES}, \text { in }}=P_{\mathrm{TES} \text {,out }}$. In order to compute 


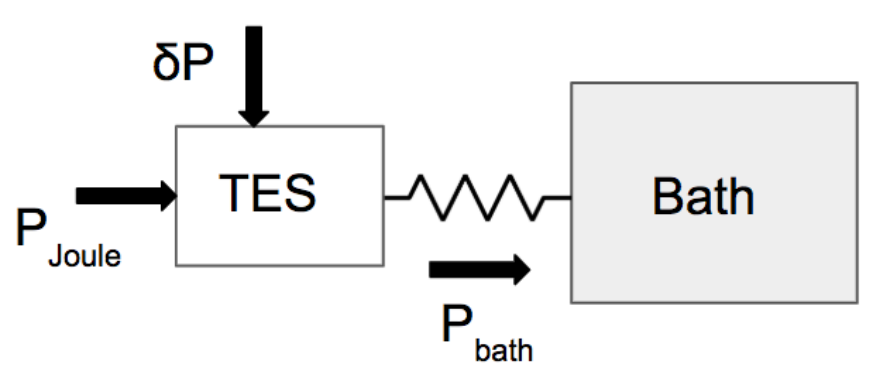

Figure 3.5: The simplified TES thermal diagram. In this simplified model, the bath represents the collection of the crystal and the dilution refrigerator. More realistic thermal models are considered in Ref. [77, 95].

these two terms we start with the simplest versions of the electric differential equation governing the TES:

$$
V_{b}=I R_{l}-L \frac{d I}{d t}+I R_{T E S}
$$

as well as the thermal differential equation governing the TES:

$$
C \frac{d T}{d t}=I^{2} R_{T E S}-P_{b a t h}+\delta P
$$

where $C$ and $T$ are the TES heat capacity and temperature respectively, the electronic component names are derived from Fig. 3.4 (right), and the thermal component names are derived from Fig. 3.5. To see how a nonequilibrium power input $\delta P$ is converted in a change $\delta I$ from the equilibrium TES current $I=I_{0}+\delta I$, we solve for the voltage across the TES:

$$
V_{T E S}=I \cdot R_{T E S}=V_{b}-I\left(R_{L}\right)+L \frac{d I_{T E S}}{d t}
$$

and plug this into the thermal differential equation, Eq. 3.6:

$$
C \frac{d T}{d t}=I \cdot\left(V_{b}-I \cdot R_{L}+L \frac{d I_{T E S}}{d t}\right)-P_{b a t h}+\delta P .
$$

We now integrate both sides of the equation over time and extend the integral to effectively infinite times (long after the TES has returned to equilib- 
rium), giving:

$$
\int_{0}^{t \rightarrow \infty} d t C \frac{d T}{d t}=\int_{0}^{t \rightarrow \infty} d t\left(I \cdot\left(V_{b}-I \cdot R_{L}+L \frac{d I_{T E S}}{d t}\right)-P_{b a t h}+\delta P\right) .
$$

Because $T$ and $I_{T E S}$ either do not change or return to their original values as the TES returns to equilibrium, the differential terms go to zero when integrated. Further rearranging so as to solve to for the circuit response to the power pulse $\delta P$ gives:

$$
\int_{0}^{t \rightarrow \infty} d t \delta P=-\int_{0}^{t \rightarrow \infty} d t\left(I \cdot\left(V_{b}-I \cdot R_{L}\right)-P_{b a t h}\right) .
$$

We also make the approximation that $P_{b a t h}$ stays at its equilibrium value as long as the TES stays in the stable regime of negative electrothermal feedback. This is a valid assumption because, when balancing a $\delta P$ in Eq. 3.6, the decrease in Joule heating $\left(\frac{V_{T E S}^{2}}{R_{T E S}}\right)$ due to the increase in $R_{T E S}$ is significantly more efficient than increasing $P_{b a t h}$. This is attributed to the fact that a change in the power dissipation to the bath requires a temperature change of the TES:

$$
P_{b a t h} \propto\left(T^{5}-T_{b a t h}^{5}\right)
$$

and as previously stated the TES does not appreciably change temperature when in electrothermal feedback. The equilibrium power dissipation to the bath is equal to the equilibrium Joule power dissipation in the TES, and therefore:

$$
P_{\text {bath }}=P_{\text {Joule }, 0}=I_{0} \cdot V_{T E S}=I_{0}\left(V_{b}-I_{0} R_{L}\right) .
$$

By Taylor expanding the TES current through the TES $\left(I_{T E S}=I_{0}+\delta I\right)$ we express the current as an equilibrium component $I_{0}$ as well as a variable component $\delta I$ that represents the dynamic signal that is measured. We also plug in for $P_{b a t h}$, and Eq. 3.10 becomes:

$$
\int_{0}^{t \rightarrow \infty} d t \delta P=-\int_{0}^{t \rightarrow \infty} d t\left(\left(I_{0}+\delta I\right) \cdot\left(V_{b}-\left(I_{0}+\delta I\right) \cdot R_{L}\right)-\left[I_{0}\left(V_{b}-I_{0} R_{L}\right)\right]\right) .
$$

Cancelling and rearranging terms gives a relationship between a displacement from the power equilibrium and the displacement from the current 


\subsection{Detector Resolution}

\begin{tabular}{c|c|c|c|c|c|c|c|c} 
& As1 & Bs1 & Cs1 & Ds1 & As2 & Bs2 & Cs2 & Ds2 \\
\hline $\mathrm{R}_{0}(\Omega)$ & 0.3 & 0.27 & 0.29 & 0.30 & 0.35 & 0.29 & 0.31 & 0.33 \\
$\mathrm{R}_{p}(\Omega)$ & 0.016 & 0.016 & 0.015 & 0.016 & 0.018 & 0.017 & 0.018 & 0.017 \\
$\mathrm{I}_{b}(\mu A)$ & 82.0 & 60.5 & 63.4 & 63.5 & 48.8 & 64.6 & 64.6 & 72.2 \\
\hline \hline $\mathrm{V}_{b}(\mu V)$ & 2.0 & 1.5 & 1.5 & 1.5 & 1.2 & 1.6 & 1.6 & 1.7 \\
$\mathrm{R}_{l}(\Omega)$ & 0.030 & 0.030 & 0.029 & 0.030 & 0.032 & 0.031 & 0.032 & 0.031
\end{tabular}

Table 3.1: Measured resistances and bias currents $\left(\mathrm{I}_{b}\right)$ by channel on the $\mathrm{S} 12 \mathrm{C}$ detector.) The values below the double horizontal line are not directly measured, but rather derived based on the schematics of Fig. 3.4, where $V_{b}=I_{b} R_{s}, R_{l}=R_{p}+R_{s}$, and the shunt resistor $R_{s}$ is taken to be $0.024 \Omega$ based on historical measurements.

equilibrium, $\delta I$ :

$$
\int_{0}^{t \rightarrow \infty} d t \delta P=-\int_{0}^{t \rightarrow \infty} d t\left[\delta I \cdot\left(2 I_{0} R_{L}-V_{b}\right)+(\delta I)^{2} R_{L}\right] .
$$

We can perform the integral effectively to $t \rightarrow \infty$ by integrating to a time after the system has returned to equilibrium. For an energy deposition in an iZIP detector, the phonon propagation and phonon collection fin coverage (discussed in Sec. 2.4) result in $\delta P$ pulse fall times of $\tau_{F} \sim 750 \mu \mathrm{s}$. Therefore, as long as we integrate to $t \gtrsim 3 \mathrm{~ms}$ we will integrate over the full time that the phonon power from the event is being dissipated in the sensor, which is equivalent to the energy from the event that is collected by the TES:

$$
E_{\text {collected }}=\int_{0}^{\gtrsim 3 \mathrm{~ms}} d t \delta P .
$$

The combination of Eq. 3.15 and Eq. 3.14 allows us to calculate the energy absorbed in the TES using the measurable electronic components $I_{0}, R_{L}, V_{b}$ as well as the TES signal $\delta I$. $E_{\text {collected }}$ can be calculated on an event-by-event basis, and the true energy of the event $E_{\text {true }}$ can be determined with an auxiliary detector calibration, and so the phonon collection efficiency can be calculated on an event-by-event basis with Eq. 3.4.

Phonon collection efficiency results for the S12C detector are shown in Fig. 3.6. A series of $<70 \%$ efficient phonon absorption processes occur between the TES and the detector which leads to the observed average $22 \%$ total collection efficiency. The lossy phonon collection mechanisms are not covered in this thesis, and are an active area of research within CDMS [95]. The final result of $\left\langle\epsilon_{\text {phonon collection }}\right\rangle=22 \%$ is invaluable in evaluating the 


\subsection{Detector Resolution}
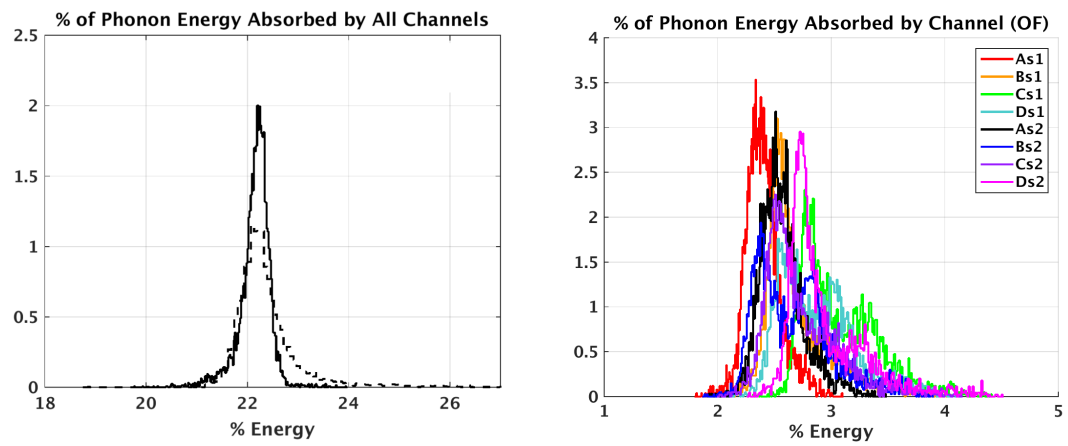

Figure 3.6: (left) The phonon collection efficiency $\left(\epsilon_{\text {phonon collection }}\right)$ calculated for events in the $100-200 \mathrm{keV}$ range, where $E_{\text {collected }}$ is calculated using the total phonon signal (the channel sum). The solid (dashed) histogram represents the $E_{\text {true }}$ measured by the optimal filter (pulse integral) energy estimate. (right) The phonon collection efficiency measured on a channel-by-channel basis. The individual S12C channels (of which there are 8 total) on average collect $2-3 \%$ of the event's energy.

detector performance because it indicates to what extent the detector resolution can be attribute to phonon collection properties vs. noise properties, which in turn informs future detector designs.

\subsubsection{Optimal Filter Baseline Resolution}

The best possible energy resolution of the TES via the optimal filter can be determined by evaluating the second derivative of the OF $\chi^{2}(a)$ amplitude fit (Eq. 2.16), which gives:

$$
\sigma_{\hat{a}}=\left[\sum_{n=0}^{N / 2}\left(2 \frac{\left|\tilde{A}_{n}\right|^{2}}{J_{n}}\right)\right]^{-1 / 2} .
$$

$\tilde{A}_{n}$ is the template and normalized such that:

$$
\sum_{n=0}^{N} A_{n}[\Delta t]=\epsilon_{\text {phonon collection }}
$$

Here $\Delta t=0.8 \mu s$ and is the time digitization rate, and we use the measured collection efficiency of $22 \%$ as shown in Fig. 3.6 left. 
For the PSD $\left(J_{n}\right)$, we use power noise as given by Fig. 3.3. This gives $\sigma=$ $51.2 \mathrm{eV}$. Comparing with measured resolution obtained via the data driven method (the $52.4 \mathrm{eV}$ shown in Fig. 3.1), offers a good cross check because these two values were arrived at relatively independently: the data-driven method depended on a calibrated energy scale, while the calculation of the theoretical resolution of the detector depended on a calibrated energy scale, a measurement of the phonon collection efficiency, as well as an accurate power noise measurement.

The utility of Eq. 3.16 goes far beyond the above example of understanding the signal and the noise contributing to the data-based resolution model. The combination of a noise estimation and phonon collection efficiency estimates with Eq. 3.16 permits projections of detector resolutions for future and existing detectors operating in ideal operating conditions. For example, in Fig. 3.7 we consider the fundamental limit of the S12C resolution operated in an ideal environment, instead of at the surface test facility where environmental noise sources abound. In this extrapolation to better operating conditions, we remove the anomalously noisy channel Cs2 from

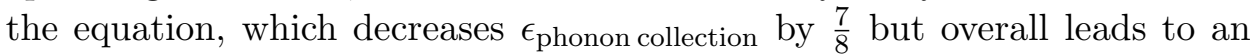
improved detector resolution as $\sqrt{J}$ is decreased by a larger fraction. The removal of this channel leads to an estimated baseline resolution of $48 \mathrm{eV}$. The next projection indicates that if we could remove the non-fundamental noise sources that generates a correlated noise component on all TESs (seen as the difference between the channel sum and the quadrature sum PSDs) then a $35 \mathrm{eV}$ threshold would be achievable on S12C. Extrapolations such as this are important for future hardware decisions, where for example we show that a $15 \%$ improvement in the baseline resolution can be achieved without any detector design changes but instead with superior environmental noise suppression strategies.

\subsection{Detector Leakage Current}

With the phonon resolution of the prototype detectors demonstrated to nearly $50 \mathrm{eV}$, our R\&D efforts turned to the high voltage (HV) operation of the prototype detectors. The CDMSlite detectors, operated as part of the SuperCDMS-Soudan payload, first demonstrated the effectiveness of applying $\mathrm{HV}(70 \mathrm{~V})$ across CDMS detectors to improve detection sensitivities to WIMPs lighter than $5 \mathrm{GeV} / c^{2}$, as shown in Fig. 2.18. For SuperCDMS SNOLAB, combining high resolution phonon sensors with HV across the detector is projected to provide sensitivities to WIMP masses below $1 \mathrm{GeV} / c^{2}$ 


\subsection{Detector Leakage Current}

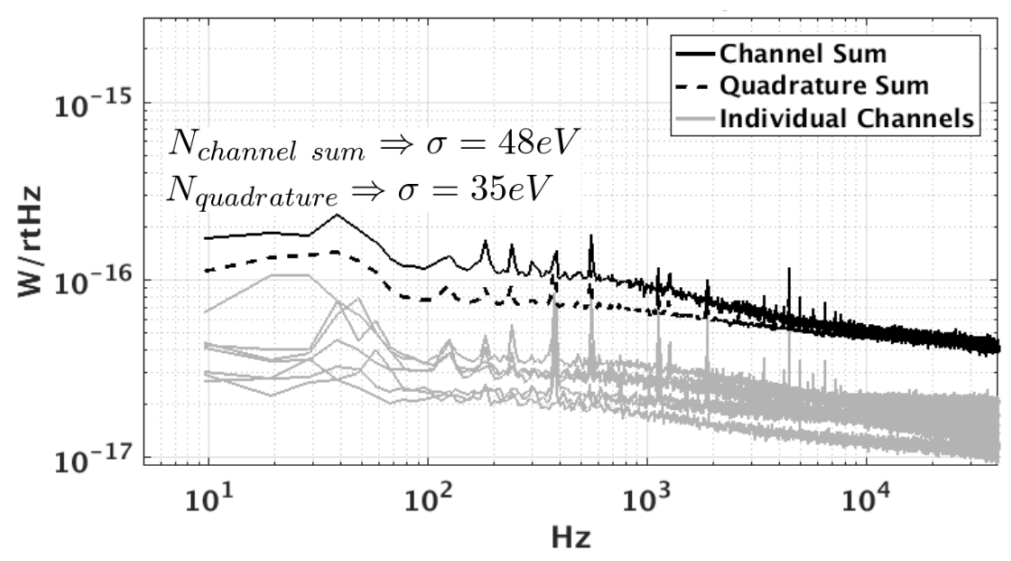

Figure 3.7: The power noise observed on S12C with channel Cs2 removed, with the detector baseline resolution (from Eq. 3.16) provided in the overlaid text.

[97]. For example, by measuring the phonon signal with a baseline resolution of $\sigma=50 \mathrm{eV}$, and operating the detector at $50 \mathrm{~V}$, the phonons from recoils that ionize just a single $e^{-} / h^{+}$pair $(<30 \mathrm{eV}$ nuclear recoils $)$ will be observed above the TES noise.

The preceding paragraph brings up the following question: since we have demonstrated that prototype detectors have reached baseline resolutions of $50 \mathrm{eV}$ (Sec. 3.1.1) and have demonstrated operation of Soudan IZIP detectors up to $70 \mathrm{~V}$ (Sec. 2.5), why have we not observed this signal from single $e^{-} / h^{+}$pairs above the TES noise? The signature of this single $e^{-} / h^{+}$pair resolution would be a quantized energy spectrum at the lowest energies, which as of yet is unobserved in the iZIP-sized detectors. The shorter answer to this question comes in two parts. First, the high sensitivity of the prototype detectors presents challenges when testing them in the high radiation environment at a surface test facility as opposed to underground at the Soudan or SNOLAB sites. Second, by operating a smaller detector that has reduced susceptibility to the radiation levels in surface test facilities, and has lower intrinsic TES noise, the SuperCDMS collaboration has observed the signal from single $e^{-} / h^{+}$pairs above the TES noise [98]. The longer explanation of the difficulties of operating of large (iZIP scale) detectors at $\mathrm{HV}$ at the surface is discussed below in the following order:

- Section 3.2.1 describes the high rate of background particles (primarily 


\subsection{Detector Leakage Current}

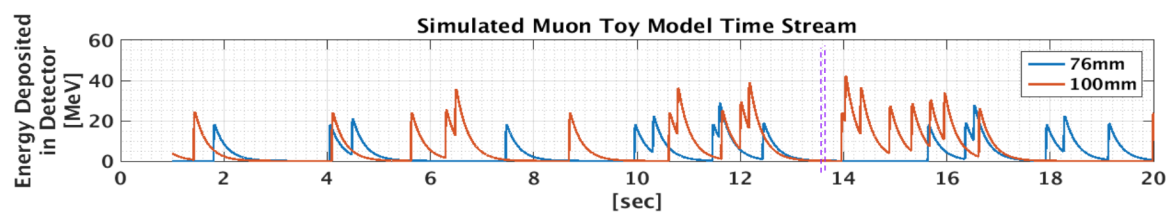

Figure 3.8: A simulated representation of the energy deposition in surface detectors with iZIP dimensions (76mm diameter) as well as for larger diameter $(100 \mathrm{~mm})$ detectors being fabricated for the SNOLAB experiment. The time window over which an event is typically digitized (50ms) is given by the time window shown by the vertical lines around 13.5 sec.

from cosmic ray muons) in the detector. These backgrounds generate a large number of $e^{-} / h^{+}$pairs in the detector.

- Section 3.2.2 describes the involved process of identifying and removing periods of time when the detector is affected by muons.

- Section 3.2.3 describes measurements of detector leakage currents, specifically:

- how the leakage current appears as an excess phonon noise in the detector

- a leakage current model, as well as a complementary simulation of the model, to confirm that the excess phonon noise that is observed comes from a leakage current

- a measurement of the leakage current on a variety of different detectors, with implications for the detector resolutions

- a discussion of leading hypotheses for the source of the leakage current.

\subsubsection{Detector Ionization Environment at Surface Facilities}

We have found that performance tests at the level of single $e^{-} / h^{+}$pair sensitivity require significant improvement of our control of background ionization in the detector when testing iZIP-sized detectors at the surface. One unavoidable background comes from cosmic ray muons that strike iZIP-sized detectors at a rate of $0.8 \mathrm{~Hz}$ with a Poisson distribution in time. Because the muons are high enough in energy that they stream through the full length of 
the detector, on average each muon deposits the same amount of energy in the detector (under the rough assumption that the muons travel vertically through the full height of the germanium). Muons traversing through the $25.4 \mathrm{~mm}$ detector height deposit $18 \mathrm{MeV}$ of energy, which translates to

$$
\frac{18 \mathrm{MeV}}{3 \mathrm{eV} \text { per e } / \mathrm{h} \text { pair }}=6 \times 10^{6} e^{-} / h^{+} \text {pairs. }
$$

For a $0.8 \mathrm{~Hz}$ muon rate, this is equivalent to a muon-induced flow of $4.8 \times$ $10^{6} e^{-} / h^{+}$pairs per second, or a current of $0.8 \mathrm{pA}$, through the detector. This is an enormous current, especially in the face of trying to observe the

signal from a single $e^{-} / h^{+}$pair. Even if the detector has the sensitivity to observe low energy events that ionize a single $e^{-} / h^{+}$pair, in practice it is made exceptionally difficult because of the muon-induced ionization that is 6 orders of magnitude larger.

Fortunately, we have the ability to identify periods during which, and shortly after, a muon has passed through the detector. Unfortunately, the iZIP detectors require approximately $250 \mathrm{~ms}$ to return to equilibrium from the thermal energy generated in the detector from a muon event. This recovery time means that there is very little time that the detector is in an operational state when high-quality data can be read out.

Figure 3.8 shows a simulation of a time series of energy deposited in the detector from muons, as a function of time, at a surface test facility. Notice that the thermal decay time from muon events $(250 \mathrm{~ms})$ is significantly longer than the athermal signal from lower energy events, and the time window over which an event is typically digitized at the surface test facilities (50 ms) is given by the time window indicated by the vertical purple lines around 13.5 seconds. As can be visualized in Fig. 3.8, there are periods of time sufficiently long after a muon event that the detector has returned to equilibrium. However, as the detector voltage is increased and the energy from the muon event generated in the detector increases linearly as a function of voltage, the amplitude of the "muon tails" grows and it becomes increasingly rare that the detector is operating in equilibrium. Analysis tools are able to identify when an event or noise trigger has been read on top of a "muon tail", and we veto these events using tools described in Sec. 3.2.2.

\subsubsection{Muon Veto Analysis Techniques}

Figure 3.9 shows real data (50 ms waveform) from random triggers from an iZIP operated at the surface, which shows large variation in the slopes of the noise traces and a large variation in the $\mathrm{DC}$ value of the traces. This 

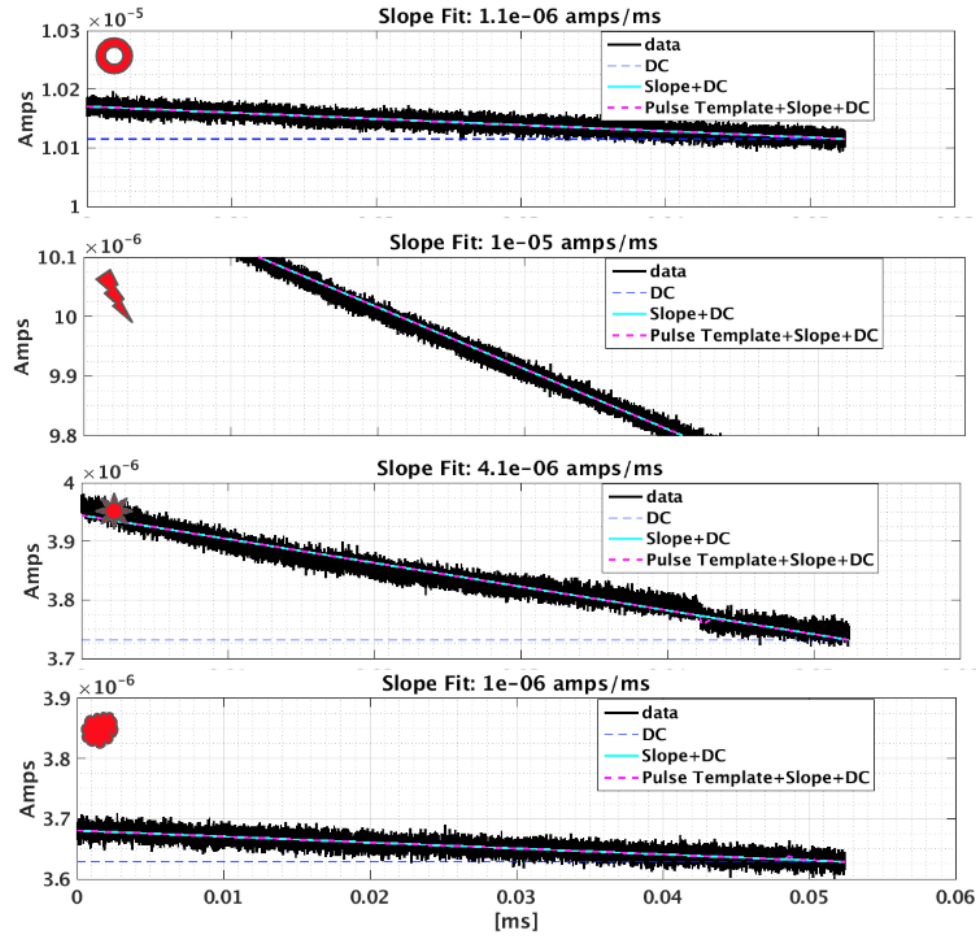

Figure 3.9: Random traces acquired on a prototype iZIP detector operated at a surface test facility, showing the slopes due to muon "tails" evident to varying degrees on top of the underlying phonon noise. The labels in the top left of the plots correspond to the labels in scatter plot shown in Fig. 3.11. 


\subsection{Detector Leakage Current}

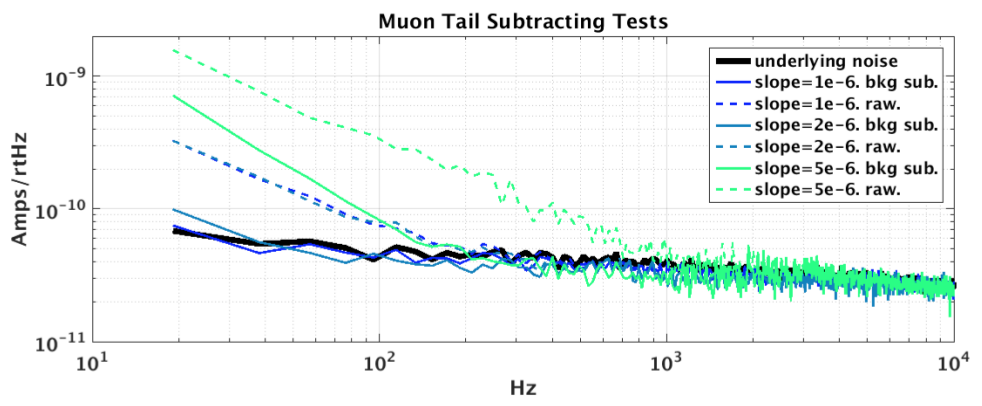

Figure 3.10: The PSD constructed with slopeless random traces (labeled as "underlying noise"). The dashed PSDs are calculated with random triggers with varying steepness of slopes (in units of amps/ms) in the randoms.

variation is to be expected based on the simulated energy deposited in an iZIP detector as a function of time shown in Fig. 3.8. The challenge posed by data of this nature is that if all the randomly acquired traces are included in the PSD calculation (Eq. 3.2), large amounts of low frequency noise will be injected into the PSDs which will be misinterpreted to be the actual underlying TES noise. This is shown directly in the PSDs in the dashed lines in Fig. 3.10, where we have selected random traces with a particular slope across them to create the PSD. Even for PSDs constructed with traces only containing modest slopes across them, for example $1 \times 10^{-6} \mathrm{amps} / \mathrm{ms}$, there is a $\times 5$ increase of the noise at $50 \mathrm{~Hz}$ as shown by the dark blue PSD vs. the "underlying" PSD that is constructed with slope-less traces.

In order to reliably measure the underlying phonon noise, some selection criteria must be applied to the random triggers prior to constructing the PSD. We have developed analysis scripts to reject randoms traces contaminated with muons, other background events, or that are otherwise anomalously noisy, by characterizing the randoms pulses with 4 quantities that provide information about the state of the detector at the time when the random was read out. These 4 quantities are:

1. The DC value of the random: if a muon has recently interacted in the detector, the $\mathrm{DC}$ value will be high

2. The slope of the random: if the detector is relaxing back to equilibrium after a muon event, there will be a significant slope across the trace

3. The optimal filter best fit amplitude $(\hat{a})$ : if a pulse exists in the ran- 
doms, the optimal filter should fit the pulse and the corresponding $\hat{a}$ should be large relative to the pulse-free random triggers

4. The optimal filter $\chi^{2}$ value: if the random is anomalously noisy or contains multiple pulses, the $\chi^{2} / \mathrm{DOF}$ will be significantly greater than 1

Figure 3.11 top shows the distribution of randoms in the DC value vs. slope plane for 5 different voltages. Based on the tolerance of the PSD to sloped randoms, an acceptance region is defined in the $4 \mathrm{D}$ parameter space, which is roughly shown as the boxed region in the DC value vs. slope plane. At low voltages, the detector is stable enough to apply the selection criteria and acquire a sufficient number of randoms $(\sim 20)$ to construct a PSD that represents the true TES noise. However, at higher voltages the distribution of random traces in the parameter space changes significantly, as shown clearly in Fig. 3.11 bottom. In particular, the necessary cut on the slope of the trace (given by the red dashed vertical line in the inset of Fig. 3.11 bottom) has an $80 \%$ random acceptance with the $0 \mathrm{~V}$ data, but a $<5 \%$ acceptance for the detector operated at $44 \mathrm{~V}$. With low cut efficiencies at high voltage, these analysis scripts have indicated that sometimes 20 minutes of data is required in order to acquire 1 second of high quality data. Despite the waiting time introduced by the muon-veto analysis techniques, they are critical for accurately measuring the TES noise PSD in a surface test facility.

\subsubsection{Phonon Noise as a Function of Detector Voltage}

Even though the muon-veto analysis scripts are effective at removing periods of high ionization in the detector, surprisingly there is a source of lower energy ionization that appears to be a continuous source of current through the detector. Up to this point we have been unable to eliminate or reliably reduce this source of ionization. We observe small numbers of $e^{-} / h^{+}$pairs leaking across the detector as a continuous source of excess power noise in the TES. The leakage becomes increasingly problematic at higher voltage because it becomes a large additional source of Luke phonons, which generates excess noise in the TES.

The excess noise is visible in the time domain, and Fig. 3.12 explicitly shows the phonon noise on two channels at $0 \mathrm{~V}$ (left) compared to $44 \mathrm{~V}$ (right). The excess noise is also clearly visible in the frequency domain, as shown for the $\mathrm{S} 12 \mathrm{C}$ detector, as well as the G23R prototype detector (which 

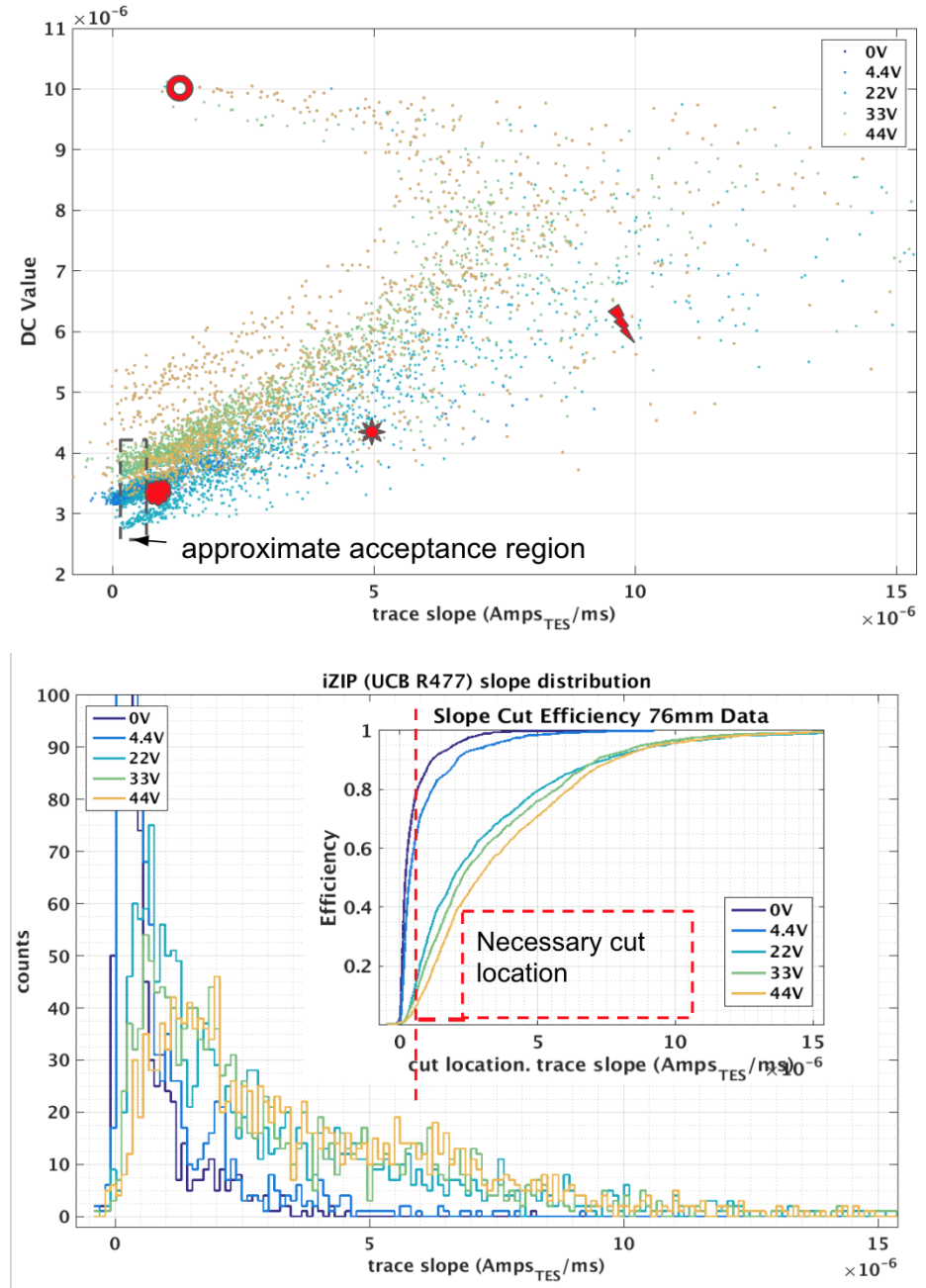

Figure 3.11: (top) The distribution of randoms in the DC value vs slope plane, showing the approximate acceptance region of this parameter space to select the random traces that are representative of the underlying TES noise. The red symbols highlight the region in this plane that the raw traces in Fig. 3.9 are selected. (bottom) A projection of the data onto just the trace slope axis, showing that the selection criteria become increasingly inefficient when the detector is operated at high voltage. 

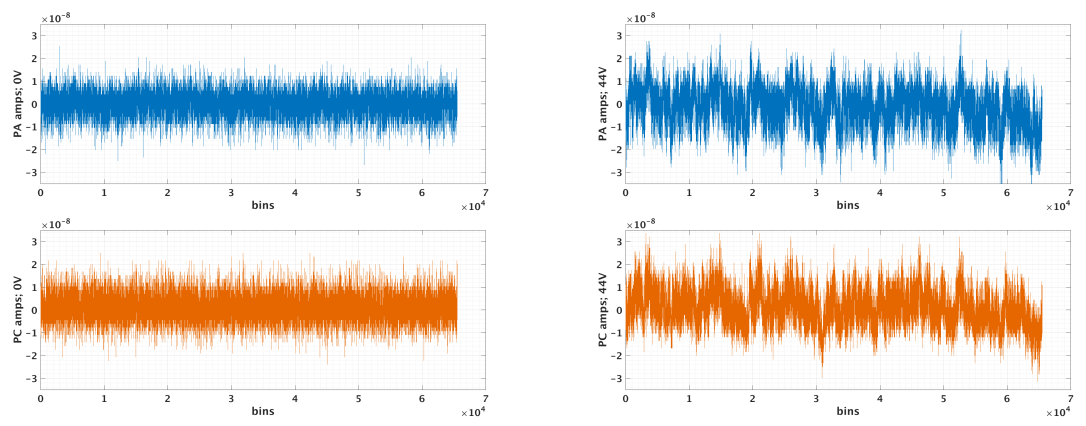

Figure 3.12: Examples of time domain noise from an iZIP-style prototype detector. The data come from two channels: channel A, top, blue and channel B, bottom, orange. The left (right) panel show noise traces from these two channels, with the detector at $0 \mathrm{~V}(44 \mathrm{~V})$. The traces read out at $44 \mathrm{~V}$ clearly have worse noise. It is also clear that the excess noise is correlated between channels, which is expected from a charge leakage noise source that stochastically generates NTL phonons distributed as small phonon pulses. These small pulses are absorbed equivalently to the standard phonon pulses on the iZIP - equally on all channels in the ballistic limitwhich leads to correlated noise. 


\subsection{Detector Leakage Current}
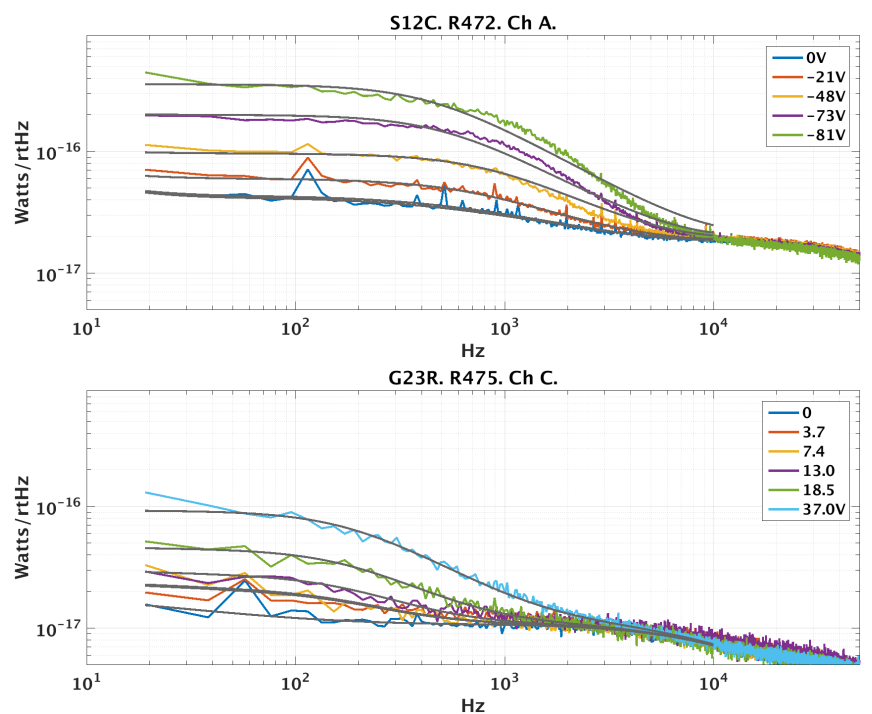

Figure 3.13: (top) Power Noise PSDs measured on a phonon channel on the $\mathrm{S} 12 \mathrm{C}$ detector (with negative HV applied to side 2, reading out side 1). Overlaid on each PSD is a leakage current model using the measured phonon fall time. (bottom) Power Noise PSDs measured on a phonon channel of the G23R prototype detector.

is also an iZIP-style detector), in Fig. 3.13. The excess noise has a characteristic shape - largest at low frequencies and negligible at high frequencieswhich is consistent with the shape of the phonon pulses (recall the pulse template curve shown in Fig. 2.6 right). This makes the excess shot noise a particularly unpleasant noise source: it has perfect overlap with the signal bandwidth.

Every detector tested at the surface exhibits this excess voltage-dependent noise, and below we motivate a physical noise model that derives the observed bandwidth of the shot noise and helps quantify the processes that are producing the excess noise.

\section{Charge Leakage Noise: Derivation of the Effect of Detector Shot Noise on TES Noise}

We refer to charge leakage noise as a "shot noise" because we use Walter Schottky's 1918 result of vacuum tube noise [99] as a starting point for a model for how individual electrons and holes traversing the detector appear 


\subsection{Detector Leakage Current}

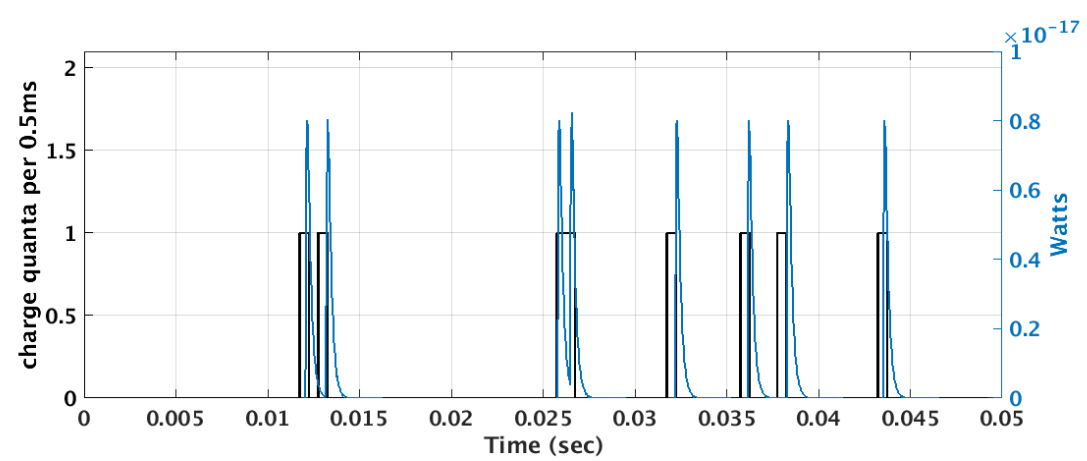

Figure 3.14: The black data depicts a time series of the shot noise model, where single charge quanta are distributed with inter-arrival times randomly sampled from a $e^{-\lambda t}$ distribution (i.e. a Poisson process). For visualization purposes the delta functions have been widened from being infinitely narrow to $0.5 \mathrm{~ms}$ wide. The average current used in this simulation was $20 \times 10^{-18}$ amps (corresponding to an average of 6.25 charge quanta per $50 \mathrm{~ms}$ interval, which fluctuated to 8 charges in this realization). The blue data depicts the phonon power generated by the charges traversing the detector, for a $50 \mathrm{~V}$ detector bias, and for a phonon absorption time of $\tau_{F}=175 \mu \mathrm{s}$ to match the $\mathrm{S} 12 \mathrm{C}$ detector.

as phonon noise in the TES. The shot noise model posits that the charges traversing the detector are delta-function quanta bursts that are Poissonian distributed in time. We denote these charge bursts as $\delta I(t) \times e$, which occur over a time length $T_{\text {trace }}$. Figure 3.14 depicts a time series of charge quanta bursts, where for visualization purposes the delta functions have been widened from being infinitely narrow to $0.5 \mathrm{~ms}$ wide.

Schottky's result shows that the current noise PSD $\left(S_{I}\right.$, in units of $\left[\mathrm{Amps}^{2} / \mathrm{Hz}\right]$ ) generated by Poisson distributed charge quanta is linearly proportional to the current $(I)$ :

$$
S_{I}(f)=2 \text { e }|I|=\frac{2 e^{2}\langle N\rangle}{T_{\text {trace }}}
$$

where $\langle N\rangle$ is the average number of charge quanta in a time series of length $T_{\text {trace }}$. Notice that $S_{I}$ is independent of frequency. The units of the right hand side are coulombs ${ }^{2} / \mathrm{sec}=\operatorname{amps}^{2} / \mathrm{Hz}$, as desired. A full derivation of this result is not given here, though the general form of this result makes sense in that Fourier transforms of $\delta I(t)$ give white noise $\left(|\delta I(\omega)|^{2}=1\right)$ and 
therefore the total noise power spectral density of these Dirac-delta pulses is also white. Additionally, it makes sense that the magnitude of the noise increases with the current (i.e. the number of charge quanta fluctuations). A full derivation of this result is given in Ref. [100].

The average rate of the dirac-delta functions (i.e. the charge leakage rate) is given by $\frac{N}{T_{\text {trace }}}=\langle\dot{n}\rangle$ which we use throughout the remainder of this derivation of the charge leakage noise model. Note that by considering just the average leakage rate, this model excludes even small cascade effects, where the leakage of a single $e^{-} / h^{+}$pair increases the breakdown likelihood of other $e^{-} / h^{+}$pairs.

Using the shot noise result, Eq. 3.19, for the noise created by deltafunction shaped charge quanta, we derive the PSD for phonon pulses with a characteristic fall time. The delta-functions of current through the detector release NTL phonons that bounce around the detector and are absorbed at the same rate as phonons from normal pulses, with the detector therefore effectively acting as a low pass filter. The filtering effect is depicted in Fig. 3.14, where the charge quanta bursts are converted to the blue $175 \mu$ s fall time pulses. The detector also acts as a current to phonon power converter by the NTL effect:

$$
\text { Phonon Power }=I \Delta V \text {. }
$$

The full current to power transfer function is then given by

$$
\left|\frac{\partial P}{\partial I}\right|=\left|\frac{\Delta V}{1+i \omega \tau_{f}}\right|
$$

where $\tau_{f}$ is the absorption time of the NTL phonon signal (also known as the pulse fall time, and $1 / \tau_{f}$ is also known as the signal bandwidth). We then convert the current noise (units of $\left[\mathrm{Amps}^{2} / \mathrm{Hz}\right]$ ) to power noise (units of $\left[\mathrm{Watts}^{2} / \mathrm{Hz}\right]$ ) by multiplying by the square of the current-power transfer function, giving:

$$
S_{P, s h o t}=\left|\frac{\Delta V^{2}}{1+\omega^{2} \tau_{f}^{2}}\right| \times e^{2} \times\langle\dot{n}\rangle .
$$

The final consideration is a correction to Eq. 3.22 for the $<100 \%$ phonon collection efficiency. Because only a small fraction of the total phonon energy is absorbed in the TESs, the power noise observed in the TES $\left(\sqrt{S_{P, \text { shot }}}\right.$, in units of $[$ Watts $/ \sqrt{\mathrm{Hz}}])$ is decreased proportionally to the phonon collection 
efficiency, $\epsilon_{\text {phonon collection: }}$

$$
S_{P, \text { shot }}(\omega)=\left|\frac{\Delta V^{2}}{1+\omega^{2} \tau_{f}^{2}}\right| \times e^{2} \times\langle\dot{n}\rangle \times \epsilon_{\text {phonon collection }}^{2} .
$$

This model predicts a specific frequency spectrum of the shot noise, and we see that the spectral shape predicted by this model exists in both simulations of detector noise and the real data, as shown below.

\section{Simulation of the Effect of Detector Shot Noise on TES Noise}

The noise model described above can be simulated on top of fundamental TES noise in order to see if the PSD of the simulated traces matches the prediction from the derivation. A depiction of this simulation is shown in Fig. 3.15.

We start with a simulation of the fundamental TES noise, without any charge leakage noise present. We then inject small pulses into the TES noise; the amplitude of the pulses is given by the voltage across the detector while the number of pulses is determined by the magnitude of the leakage current. The fall time of the pulse is given by the phonon collection time (i.e. the fall time of the phonon pulses) and in this simulation we use $\tau_{f}=175 \mu$ s to match the collection time observed on the S12C detector. In Fig. 3.15, the green pulse shows one of the charge leakage pulses for a detector voltage of $50 \mathrm{~V}$ (multiplied by 10 in order to be visible). We them sum 6250 of these pulses together, Poisson distributed over the $52 \mathrm{~ms}$ simulated time series (which corresponds to the number of charge leakage for a leakage current of $0.02 \mathrm{pA}$ ), and the result of summing many of these pulses together is given by the red curve. Adding the fundamental TES noise gives the blue curve.

We simulate a collection of the TES plus shot noise time series at a particular voltage, and then compute their PSD. We repeat this for voltages $0 \mathrm{~V}, 10 \mathrm{~V}, 20 \mathrm{~V}, \ldots 100 \mathrm{~V}$ to see how the noise changes in the frequency domain as a function of voltage, and the results are shown in Fig. 3.16. Notice that the excess noise shape observed in the simulation matches the spectral shape observed in the data for the S12C detector (Fig. 3.13 top) which has the same signal bandwidth used in the simulation.

Notice also, in Fig. 3.13, the excess noise spectral shape change is different between the S12C and G23R detectors, and this spectral difference is predicted from the model of Eq. 3.22. The noise moves to higher frequencies from G23R (which has a fall time of $\tau_{f}=875 \mu \mathrm{s}$ ) to S12C (which has a fall time of $\left.\tau_{f}=175 \mu \mathrm{s}\right)$, which is the trend predicted by the model. The 


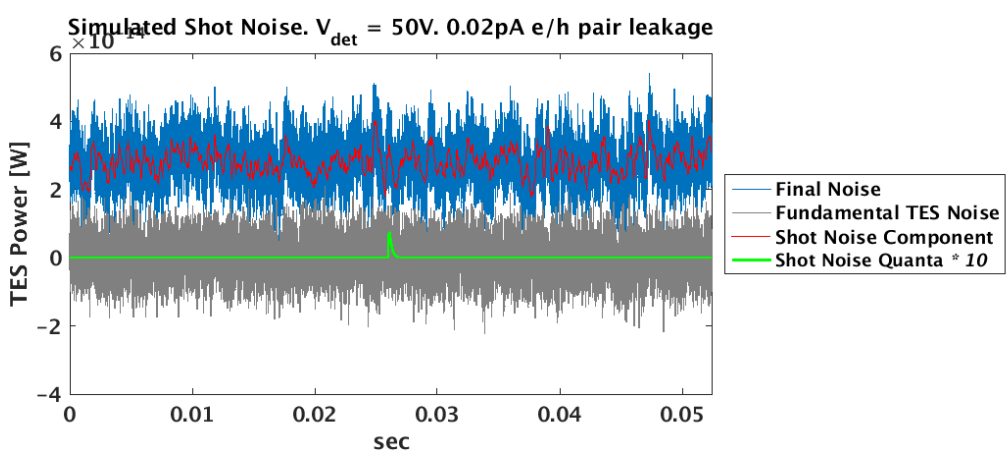

Figure 3.15: Example of the simulation of a single $50 \mathrm{~ms}$ data stream of charge leakage shot noise injected onto fundamental TES noise. The green pulse is amplified by a factor of 10 (in order to be visible) and its fall time is $\tau_{f}=175 \mu$ s to match the $\mathrm{S} 12 \mathrm{C}$ detector. Many of the pulses are Poisson distributed in time and added to the fundamental TES noise, giving the blue curve.

spectral shape predicted by the model is specifically overlaid as grey lines in Fig. 3.13, which fits the data well except for on S12C at the highest voltages. The best explanation for this behaviour only at the highest voltages is that the excess power from the NTL phonons generated by charge leakage is changing the dynamic response of the TES and changing the pulse fall time slightly from $\tau_{f}=175 \mu \mathrm{s}$.

\section{Calculation of Leakage Currents}

The excess TES noise is used to calculate the leakage current for the S12C detector at a variety of voltages. We solve for the charge leakage rate, $\dot{n}$, in Eq. 3.23, where for $S_{P, s h o t}(\omega, V)$ we calculate the contribution of the shot noise by subtracting the $0 \mathrm{~V}$ noise from the total noise:

$$
S_{P, \text { shot }}(\omega, V)=S_{P, \text { total }}(\omega, V)-S_{P, \text { total }}(\omega, V=0) .
$$

We solve for $\dot{n}$ and other quantities relating to the detector leakage current, as a function of voltage using the PSD curves shown in Fig. 3.13 top, in Table 3.2.

The leakage current is calculated for a variety of detectors on which an excess noise was found as a function of voltage applied across the detector, as shown in Fig. 3.17. The error bars include uncertainties in the measurements of power noise as well as uncertainties in the voltage applied across 


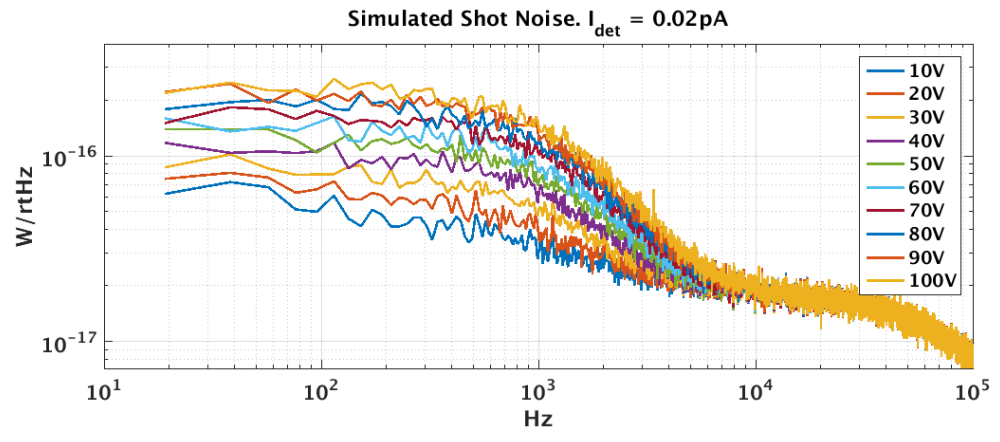

Figure 3.16: PSDs are made of the simulated traces using different detector voltages in the simulations. The noise shape and magnitude are consistent with those predicted by the shot noise model (Eq. 3.23), as well as consistent with the noise shape seen in the data (e.g. Fig. 3.13).

\begin{tabular}{c|c|c|c|c} 
Voltage $(\mathbf{V})$ & $S_{P, \text { total }}(\omega=0, V)$ & charges $/ \sec (\dot{n})$ & $I$ (amps) & $R_{\text {detector }}(\Omega)$ \\
\hline 0 & $1.6 \times 10^{-33}$ & $\sim 0$ & $\sim 0$ & $\sim \infty$ \\
10 & $1.6 \times 10^{-33}$ & $\sim 0$ & $\sim 0$ & $\sim \infty$ \\
21 & $3.6 \times 10^{-33}$ & $1.2 \times 10^{5}$ & $1.5 \times 10^{-14}$ & $1.1 \times 10^{15}$ \\
48 & $1.0 \times 10^{-32}$ & $9.4 \times 10^{4}$ & $1.5 \times 10^{-14}$ & $3.2 \times 10^{15}$ \\
73 & $4.4 \times 10^{-32}$ & $2.1 \times 10^{5}$ & $2.3 \times 10^{-14}$ & $2.2 \times 10^{15}$ \\
81 & $1.2 \times 10^{-31}$ & $4.7 \times 10^{5}$ & $7.6 \times 10^{-14}$ & $1.1 \times 10^{15}$
\end{tabular}

Table 3.2: The leakage current, as well as the effective total ohmic detector resistance, derived from the listed excess noise levels at low frequency on the $\mathrm{S} 12 \mathrm{C}$ detector. 


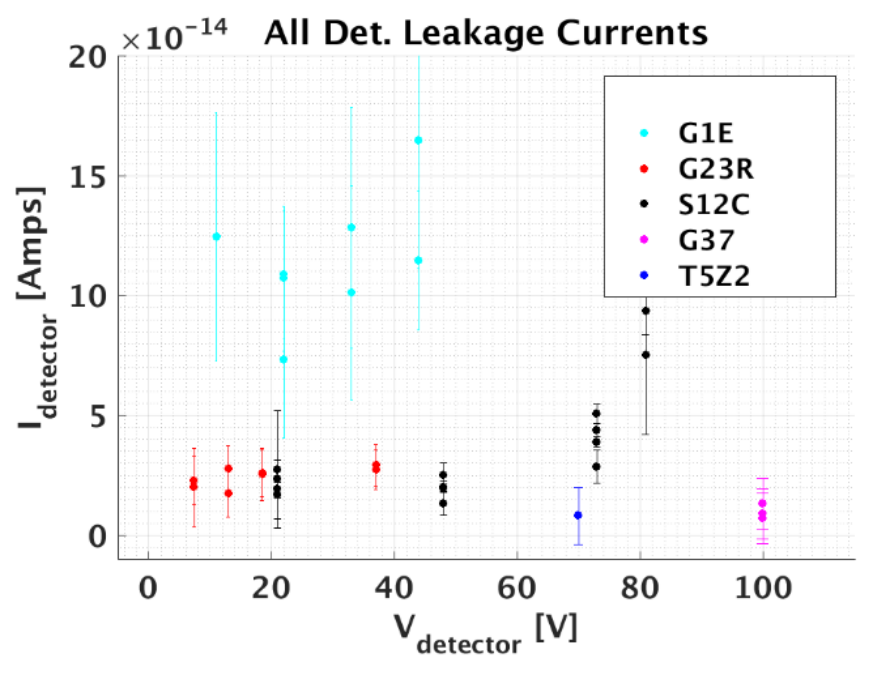

Figure 3.17: Leakage current measurements, as a function of voltage, for a number of detectors (primarily prototype, primarily iZIP style and size). All detectors here were measured at a surface test facility, with the exception of the blue "T5Z2" data point. This data point is determined using data acquired from Soudan, but is a rough estimate for a number of reasons, one of which is that low frequency noise present in all PSDs from Soudan masked the shot noise signal.

the detector. Examining just the S12C data points, it aligned with expectations that at some voltage the current increased significantly (as observed at $\sim 80 \mathrm{~V}$ ) based upon the idea of "breakdown," where above some voltage the Coulomb barriers of the detector interface are overcome and a current flows. The more surprising finding from this study was the leakage current behaviour at low voltage, showing a nearly voltage-independent magnitude. This $I-V$ relationship was seen on a number of the other tested detectors, and has implications for the detector resolution, which are discussed in the section below.

\section{Effect on Detector Resolutions}

The primary problem with the leakage current is that it negatively affects the detector baseline resolution. The principle of NTL amplification, as shown in Fig. 2.15 center, is that the signal increases linearly with the voltage and the 

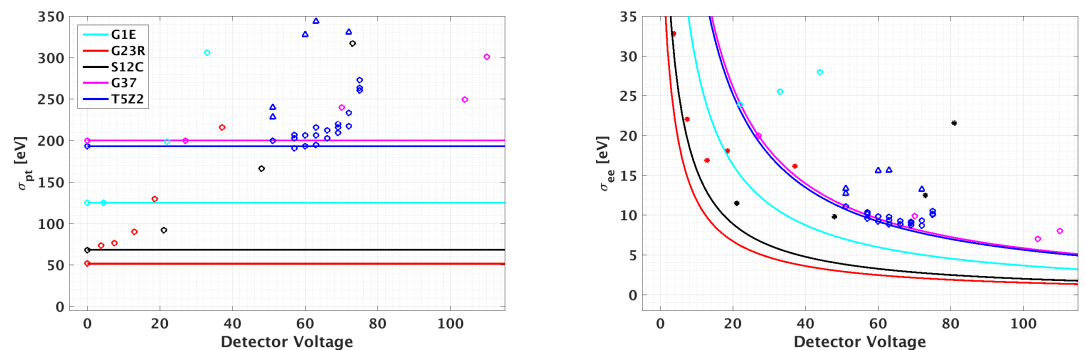

Figure 3.18: (left) The total phonon baseline resolution $\left(\sigma_{\mathrm{t}}\right)$ for a number of detectors as a function of detector voltage. The solid lines express ideal (no leakage current) resolution scalings as a function of voltage where the $\sigma_{\mathrm{t}}$ value remains constant as a function of voltage. The data points represent resolution measurements at those voltages, or deduced resolutions based on PSD measurements and Eq. 3.16. (right) The electron equivalent energy resolution $\left(\sigma_{\mathrm{t}}\right)$ as a function of voltage, derived from the left hand plot using Eq. 3.26.

noise remains constant until some "breakdown" value. With constant noise vs. detector voltage, the baseline resolution will be constant as a function of voltage when measured in units of total phonon energy. As described in Sec. 2.5, the NTL amplification of the signal is linear as a function of voltage:

$$
E_{\mathrm{t}}=E_{\mathrm{ee}}\left(1+\frac{e V_{\mathrm{b}}}{\epsilon}\right)
$$

so that when the baseline energy resolution is measured in electron equivalent energy units, it is inversely proportional to the voltage bias:

$$
\sigma_{\mathrm{ee}}=\frac{\sigma_{\mathrm{t}}}{1+e V_{\mathrm{b}} / \epsilon} \text {. }
$$

When there is constant noise vs. voltage the $\sigma_{\mathrm{t}}$ resolution is constant, and the solid lines in Fig. 3.18 show this ideal baseline energy resolution scaling. When that resolution is converted to the electron equivalent energy scale $\left(\sigma_{\mathrm{ee}}\right)$, the resolution is inversely proportional to the detector voltage, as shown in Fig. 3.18 (right).

When the noise increases as a function of voltage, the $\sigma_{\text {ee }}$ baseline resolution will improve more slowly as function of the detector voltage than indicated by the solid lines of Fig. 3.18. For the data points in Fig. 3.18 left, we use Eq. 3.16 to calculate the total phonon energy detector baseline resolution $\sigma_{\mathrm{t}}$, using the observed noise $J_{n}$ as a function of voltage. For $V_{\text {det }}>0$ 
the data points are above the ideal resolutions because the noise increases as a function of voltage.

\section{Current Source Hypotheses}

Up to this point we have not been able to reduce the shot noise induced by the leakage current nor identify the source of the ionization. We have robustly demonstrated that infrared radiation (IR) is not the cause. To eliminate low energy ionization due to any infrared background at the detector, IR blocking "Bock Black" paste developed by the millimeter astronomy community [101] was applied to the detector lid in between measurements of the leakage current, and no change in the leakage was observed. Other hypotheses for the leakage currents are leakage from the instrumented surface of the detector, and leakage from the detector bulk from impurities in shallow potential wells hopping into the conduction band. This bulk leakage model would be akin to the leakage that is observed in the prebiasing procedure described in Sec. 2.5.3 and discussed further in the Appendices of Ref. [83]. An additional hypothesis relates to the unstable voltage across the detector due to the large amount of ionization generated from muon events. It is of course possible that the leakage current is a combination of multiple sources, which may make it particularly difficult to diagnose. Subsequent to the measurements made here, additional insights into the combination of the contributions to the leakage current have been made on the smaller scale quantization detector [98] with improved control of systematic errors from the smaller detector area.

Fortunately, many of the mechanisms that would generate the significant leakage currents shown in Fig. 3.17 would be reduced when operating the detector underground. An additional piece of evidence that suggests that the leakage current is suppressed for underground detector operation

is presented in the following chapter. This chapter contains analysis of data from the T2Z1 detector operated at Soudan in 2015 during which the detector voltage varied between $50 \mathrm{~V}$ and $75 \mathrm{~V}$. While the low frequency noise present at Soudan limited abilities to study the voltage dependent shot noise, Sec. 4.3.4 presents a study of the trigger rate on T2Z1 and shows that below $72 \mathrm{~V}$ the detector operation was stable and the trigger rate effects due to noise were exponentially decreasing below $72 \mathrm{~V}$. 


\section{Chapter 4}

\section{Data Analysis of CDMSlite Run 3}

\subsection{Motivation}

As the final CDMSlite run at Soudan, the CDMSlite Run 3 analysis served as a test-bed for many techniques that are being considered for the SNOLAB science analyses, and we discuss three of these new techniques in this chapter. First, a blinding technique was used for the first time in CDMSlite as a method to reduce the possibility of analyzer bias. Second, advanced multivariate discrimination techniques were used to efficiently remove instrumental backgrounds at the lowest energies measurable by the detector, which were a limiting background for the Run 2 analysis. Third, a Monte Carlo modeling of the detector fiducial volume was used to inform our "radial cut" which removed misreconstructed events at high detector radii.

All of the above techniques contributed towards the larger goal of moving away from the optimum interval limit-setting approach, discussed in Sec. 2.5.3, towards a more powerful limit-setting approach. Successful implementation of the above techniques allowed us to accomplish that goal and the following chapter, Chapter 5 , discusses the details of the limit-setting approach used for the Run 3 analysis. This method had the benefit of moving CDMSlite from an exclusion-only analysis into one with discovery potential, and also the benefit of improving the limit over the optimum interval method.

In addition to the new techniques brought to the Run 3 analysis discussed in the following chapter, a number of the techniques employed are the same as those used in the Run 2 analysis. In particular, we use the same method of pulse simulation to measure cut efficiency. We also use the same method of discriminating against the more obvious instrumental noise types with optimal filter fits using instrumental noise templates. Additionally, the fiducial volume Monte Carlo built on the radial parameter successes from the Run 2 analysis. 


\subsection{Salting}

Prior to the start of the CDMSlite R3 analysis, the collaboration decided to adopt a blinding strategy to prevent analyzers from making biased decisions in order to reach a desired result. Because this was the first time that a CDMSlite analysis would be performed blind, a blinding task force was formed to consider a variety of blinding schemes. These included data division, where a fraction of the events are used to tune cuts, develop background models, etc. and the remaining events are used to search for a DM signal. "Box blinding" was also considered, where the region of the parameter space where the signal is expected is hidden from the analyzers and only opened once analysis decisions are finalized using information outside of the blinded region.

Ultimately these two blinding schemes were determined to be ill-suited for CDMSlite because of instrumental noise events that exist in the data. Instrumental noise events make up the majority of events and their magnitude and characteristics change as a function of time. Therefore it is desirable to be able to see all events at each stage of the analysis and so, rather than hiding events, the blinding task force implemented data "salting" in which a fraction of the events in the dataset were replaced with artificial signal-like events. This procedure effectively masked the true amount of DM signal in the data (i.e. it is "blinding with a flashlight").

The salting procedure was developed openly in that the algorithm was known to the CDMSlite Run 3 analyzers, and therefore CDMSlite analyzers were able to contribute to parts of the salting development. In Sec. 4.2.1 and 4.2.2, I describe my primary contributions to the salting effort. This open development of the salting algorithm did not violate blinding because the salting algorithm was designed such that the salted events had a normalization and energy spectrum that was randomized within some pre-defined range.

\subsubsection{Determining the Target Number of Salt Events}

There is not an obvious number of artificial events to insert into the dataset in order to accomplish the goal of reducing analyzer bias. This section explains how the number of artificial events was chosen non-arbitrarily.

First, there should be enough salt that analyzers will be wary of tuning cuts to remove events, while too much salt would potentially mask instrumental backgrounds that we want to be able to identify and remove with cuts. The simplest choice of the number of salt events is some fraction of 


\subsection{Salting}
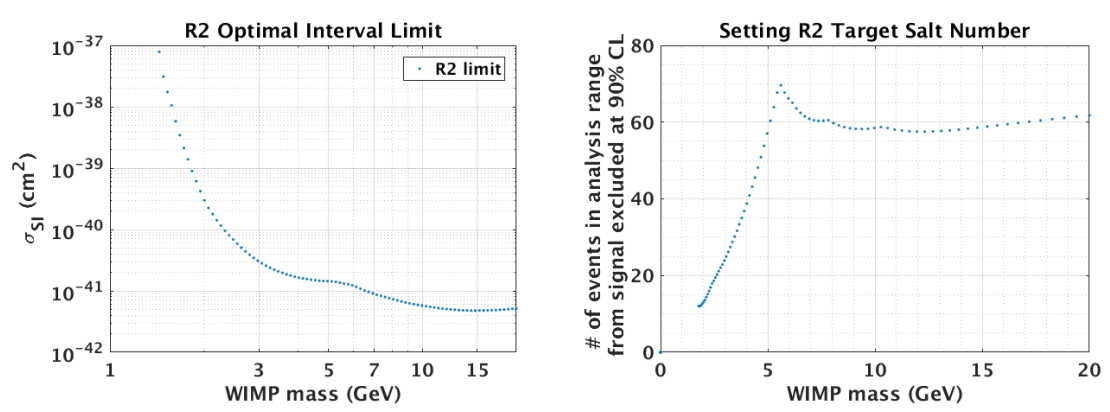

Figure 4.1: (left) The CDMSlite Run 2 90\% CL OI exclusion spin- independent cross section limit as a function of DM mass. (right) The number of DM events that correspond to the excluded $\sigma_{S I}$ from the left figure.

the expected number of events in the analysis's region of interest. However, this option proved to be inadequate for CDMSlite because the vast majority of events in the region of interest are concentrated in a narrow energy range from the ${ }^{71} \mathrm{Ge}$ decays. Instead of the ${ }^{71} \mathrm{Ge}$ peaks dictating the number of artificial events added, the energy ranges where there is a lower level of expected background should dictate the number of events added. This is because these are the energy ranges where the dataset has the highest sensitivity to signal and therefore where it's most important to have an appropriate amount of salt.

Instead of using a fraction of the expected number of events in the analysis's region of interest, the projected optimum interval sensitivity of the analysis can be used to naturally incorporate the energy region of low background when choosing the number of artificial events. Because the optimal interval method's sensitivity generally is determined from an energy range in the final spectrum where there is a low background rate, this method will not be susceptible to the potential problem of a large fraction of events appearing in the ${ }^{71} \mathrm{Ge}$ peaks.

For salting CDMSlite Run 3, we estimate the sensitivity by using the CDMSlite Run 2 OI cross section limit. The cross section limit can be converted into a number of excluded events using the exposure (i.e. the $\mathrm{kg} \times$ days length of the run) as a function of the DM mass, and is shown in Fig. 4.1.

The $20 \mathrm{GeV} / c^{2}$ WIMP spectrum covers the full energy range of interest for the analysis, and therefore we base the salt normalization off of this WIMP mass, which gives 62 events at the excluded limit. A visualization of this number of artificial events, given energies drawn from an exponential 


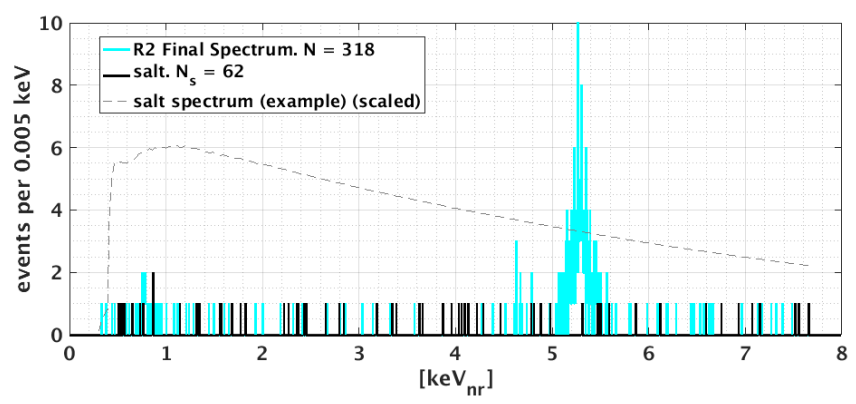

Figure 4.2: Example "salt" added to the CDMSlite Run 2 final spectrum at the magnitude indicated by the excluded $90 \%$ CL for a DM signal. The salt spectrum is scaled to a much higher level than the exclusion limit to illustrate its shape.

distribution with a constant offset, is shown in Fig. 4.2 along with the final Run 2 spectrum. This visualization shows that inserting events at level of the $90 \%$ OI CL sensitivity gives a reasonable number of events: the artificial event density is sufficient to prevent cut tuning but not so large as to mask the real events in the regions of low background or potentially mask an unanticipated or instrumental background in the CDMSlite Run 3 dataset. This method of determining the number of salt events based on the sensitivity was adopted because it reduces the arbitrary nature of the choice.

The number of salt events to use for Run 3 was determined based on the 62 events calculated from Run 2, but scaled by the relative duration of the runs, corrected by the estimated passage fraction of the salt to analysis cuts $(\sim 15 \%)$, and also randomized within a range of possible values. The final range was 280-840 events, and the number of salt events used for Run 3 was revealed to be 393 after the dataset was unblinded.

\subsubsection{Selecting Events To Replace}

The salting algorithm was designed to replace events with artificial events, rather than adding artificial events, for technical simplicity. This was in part to avoid having to work around the sequential event IDs that are a feature of the CDMS data format. Each CDMS event also contains a significant amount of metadata in addition to the digitized waveforms (e.g. cryocooler phase information, base temperature information, trigger time stamps, de- 
tector array trigger information). Trying to appropriately generate artificial data for all these categories would be a challenge, and so instead the existing algorithm only creates artificial waveform data and otherwise inherits the metadata from the replaced event.

While the event replacement approach had many advantages, it required particular attention when selecting which events to replace. Of course the replaced events were chosen to be uniformly distributed in calendar time (as expected of a DM signal). In other variables it would be less easy to replace events in such a way that the artificial events resemble the expected distributions of DM signal events. For example, the artificial events should be uniformly distributed with the cryocooler vibrational phase, but because as shown in Fig. 4.6 the majority of events occur within a certain range of the cryocooler vibrational phase, additional analysis selection criteria were required when choosing the events to replace.

During inspection of the first attempt to salt the CDMSlite Run 3 dataset, the salt events were found to be not uniform in cryocooler vibrational phase but highly correlated with the cryocooler-induced noise triggers. Events that are reconstructed at higher energy are less likely to be low frequency noise, as shown in Fig. 4.3, and so the undesired correlation was removed by raising the minimum energy of the replaced events. Fig. 4.3 indicates that an energy cut of $2 \mathrm{keV}_{\mathrm{t}}$ is sufficient to remove the correlation,

and because the low frequency noise was found to get worse after the ${ }^{252} \mathrm{Cf}$ calibration data shown in the figure, the final energy cut was set at $3 \mathrm{keV}_{\mathrm{t}}$.

\subsubsection{CDMSlite R3 Salt Application and "Unsalting"}

In the final application of the salting algorithm, the event energies were chosen from an exponential distribution with a constant offset:

$$
P(E) \propto C+(1 / D) \exp ^{-E / D} ; \quad E \in[0.05,5] \mathrm{keV}_{\mathrm{ee}},
$$

where the exponential component was chosen to roughly approximate a WIMP spectrum and the constant offset was chosen so that salt existed over the analysis energy region of interest. $C$ and $D$ were randomized hidden parameters, sampled logarithmically from $1 / 3$ to $3 \mathrm{keV}_{\mathrm{ee}}^{-1}$ for $C$, and from 0.5 to $2 \mathrm{keV}_{\text {ee }}$ for $D$. The chosen energy was restricted from 0.05 to $5 \mathrm{keV}_{\mathrm{ee}}$ to match the analysis's region of interest. The randomly selected parameters used were $C=0.6967 \mathrm{keV}_{\mathrm{ee}}^{-1}$ and $D=1.299 \mathrm{keV}_{\mathrm{ee}}$, resulting in a nearly uniform distribution of salt events over the energy region of interest.

The success of the salting procedure was confirmed in a post-unblinding analysis effort. The salt was inserted at an appropriate magnitude and as- 


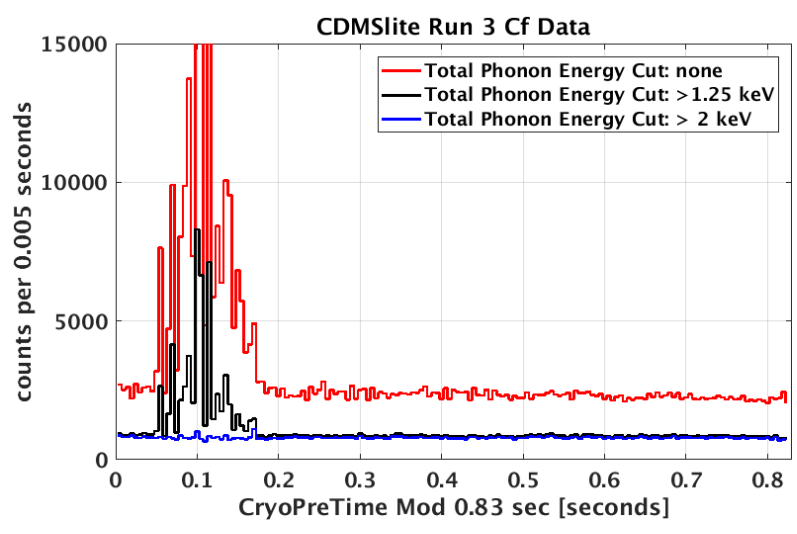

Figure 4.3: The distribution of ${ }^{252} \mathrm{Cf}$ calibration data in the crycooler vibrational phase variable (labeled as CryoPreTime Mod 0.83s). As the selection criteria moves to higher energy, the correlation of events with the phase decreases.

sumed a signal-like distribution in the parameters of interest for the analysis. Details of these post-analysis confirmations are documented in the results section of the following chapter, in Sec. 5.3.2.

\subsection{Experimental Setup}

A comprehensive review of the CDMSlite experimental setup in the context of the Run 1 and Run 2 analyses can be found in the theses of R. B. Thakur and M. Pepin $[67,83]$. Therefore, this section primarily highlights the experimental setup differences between Run 3 and the earlier CDMSlite runs.

\subsubsection{Detector Selection and Configuration}

Figure 4.4 shows the SuperCDMS Soudan detector array with the T5Z2 and T2Z1 detectors labeled. The decision to switch CDMSlite detectors from T5Z2 to T2Z1 for Run 3 was based on a number of factors. First, a better noise environment was observed on T2Z1, relative to T5Z2 and most other detectors, leading to a slightly better baseline resolution than that observed with T5Z2. The superior noise performance was primarily because of reduced susceptibility to vibrational noise as discussed in Sec. 4.3.2. We expected this would enable a lower analysis threshold for Run 3 and an improved sensitivity to low-mass DM. In addition, during Run 3 commis- 

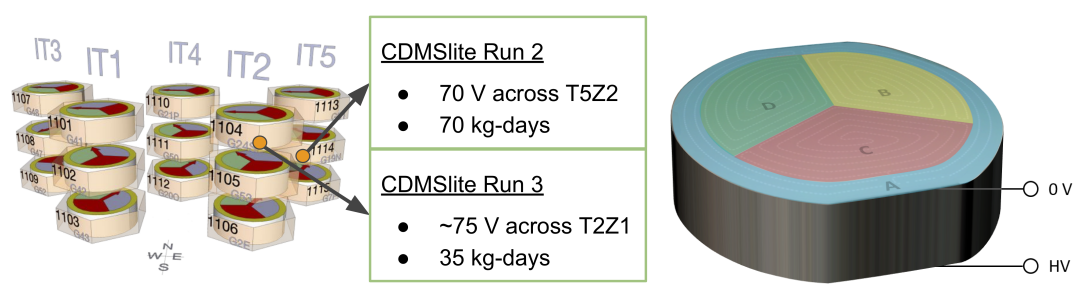

Figure 4.4: (left) A depiction of the SuperCDMS Soudan detector array, with 15 iZIPs total arranged in 5 "towers." (right) A depiction of an iZIP detector read out in CDMSlite mode. Copied from Ref. [82], with permission.

sioning, we saw that the measured leakage current for T2Z1 was less than that of other candidate detectors. And finally, the decision to switch from T5Z2 to T2Z1 for Run 3 was intended to demonstrate reproducibility of the CDMSlite operating technique across multiple detectors.

Side 2 of T2Z1 was biased with a 75V applied voltage while the phonon and charge channels on side 1 were read out. As discussed in Sec. 4.3.3, and unlike in Run 2, there were times throughout Run 3 that the detector voltage differed significantly from the applied power supply voltage. The data acquisition readout for Run 3 was also limited to a single tower (Tower 2) as opposed to previous CDMSlite runs when the whole detector array was read out.

The pre-biasing procedure - where the detector voltage is raised above the operating voltage for 10 minutes before data taking as discussed in Sec. 2.5.3 - was found to reduce charge leakage shot noise in the phonon signal at the start of each data series. For Run 3, 85V was used as the prebias point for the nominal $75 \mathrm{~V}$ operating voltage. (In Run 2, a $80 \mathrm{~V}$ prebias was used for the Run $270 \mathrm{~V}$ operating voltage).

\subsubsection{Vibration Monitoring}

One of the central challenges to all low threshold SuperCDMS Soudan analyses is the significant contribution of "low frequency noise" (LFN) to the noise budget of the phonon readout. This noise source affects all Soudan detectors, degrades detector baseline resolutions, forces the setting of higher analysis thresholds, and as a result reduces sensitivity to low mass WIMPs. Section 2.5.3 describes how, prior to CDMSlite Run 2, accelerometers were installed on the cryocooler, which was the most vibrationally intensive component of the experiment. 


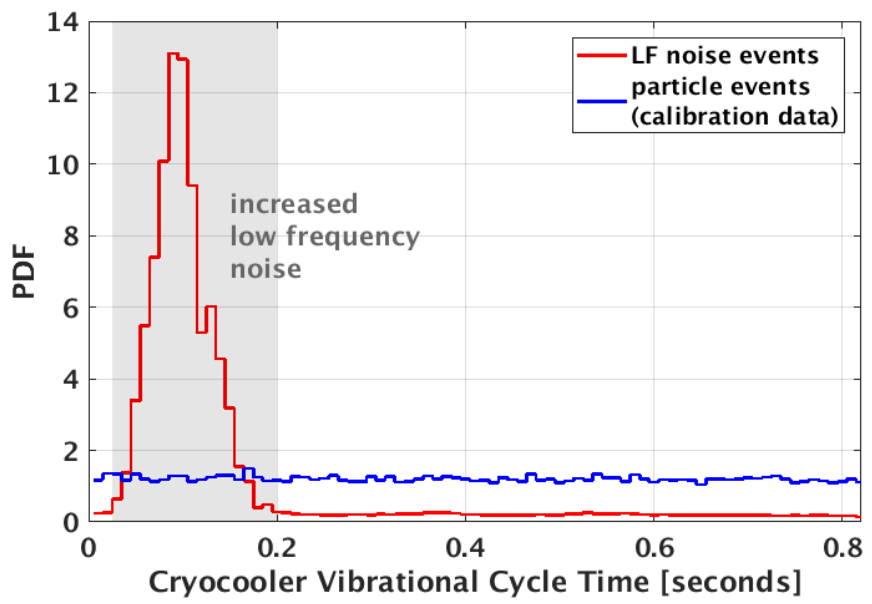

Figure 4.5: The low energy event density dependence on the the cryocooler vibrational phase is shown in the red histogram. Good events from actual energy depositions, shown in the blue histogram, are not correlated with the vibrational phase.

The most useful cryocooler correlation analysis variable, discovered during the CDMSlite Run 2 analysis, was the "time since" last cryocooler chirp, where the cryocooler chirps were found to occur at 0.83 second intervals [102]. Because on occasion the accelerometer did not register a cryocooler chirp and a period lasted $n \times 0.83$ seconds, the $0.83 \mathrm{~s}$ modulus of the cryocooler "time since" variable proved the most beneficial analysis variable for Run 2 and Run 3 analyses.

Because the LFN caused triggers that could mimic low energy events $\left(<5 \mathrm{keV}_{\mathrm{t}}\right)$, and because the LFN caused more triggers than any other background source in Run 3, we use the low energy event density as a proxy for the intensity of the LFN. Figure 4.5 shows the low energy event density dependence on the time since last cryocooler chirp (also known as CryoPreTime Mod $0.83 \mathrm{~s}$, or more generally as the cryocooler phase). The LFN vibrations on T2Z1 are strongest 0.1 seconds after a crycooler cycle. Section 4.6 describes how we incorporate this information into overall LFN quality cuts.

The vibrational noise from the cryocooler was also found to vary over the course of the $\sim 60$ day dataset, as shown in Fig. 4.6. In addition to the variable event rate in the $0-0.2 \mathrm{~s}$ range of the crycooler phase, an additional 


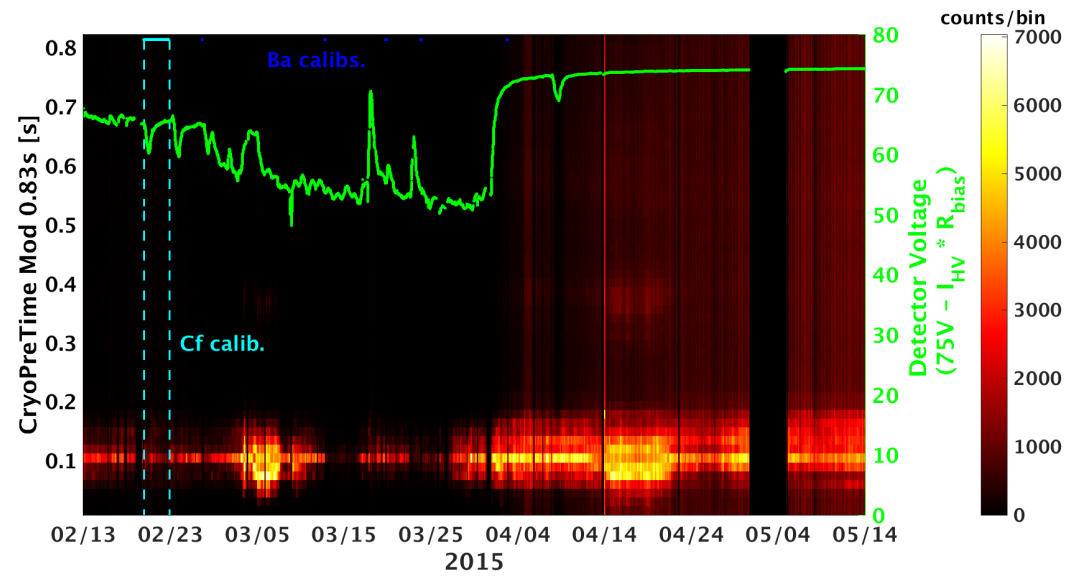

Figure 4.6: An event density plot of lower energy events $\left(-2<\mathrm{keV}_{\mathrm{t}}<5\right)$ that are more likely to be instrumental noise events than events from particle energy depositions in the detector. Ba and Cf calibrations are highlighted. A Cf period when no data was read out is between May 01 and May 05. The April 14 stripe is from a single series (01150414_1430) when the cold trap was being cleaned. A second 3 hour series on May 05 (01150505_1422) was removed from the plot as it was saturating the color map - this was the first series after the late-Run Cf calibration.

noise source that is uncorrelated with the crycooler vibrational phase was observed to start on roughly April 1, and is explored below in Sec. 4.3.3.

\subsubsection{High Voltage Current Monitoring}

Monitoring of the HV power supply current began with CDMSlite Run 2, and the motivation and basic aspects of this current reading are described in Sec. 2.5.3. The HV current readings were critical for correcting the reconstructed energy of events in the Run 3 analysis. Referring to the schematic shown in Fig. 2.16, the value of $\mathrm{R}_{p}$ (and therefore $I_{H V}$ ) varies significantly over time, between an effectively infinite resistance and $625 \mathrm{M} \Omega$. As shown in Fig. 4.7, $\mathrm{R}_{p}$ was found to be strongly correlated with the temperature in the room that housed the electronics (the RF room). Because this variation is undesirable, the air handler which supplied fresh air to the RF room was turned off in early April 2015, raising the temperature in the room and increasing the parasitic resistance (decreasing the leakage current). 


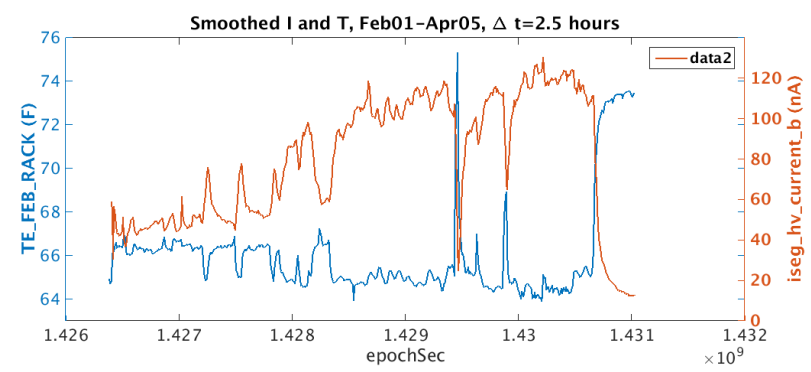

Figure 4.7: The RF room temperature (TE_FEB_RACK) $\left({ }^{\circ} \mathrm{F}\right)$ and the leakage current (iseg_hv_current_b) (nA) as a function of calendar time. The $\mathrm{x}$-axis runs from mid-February to early April, 2015.
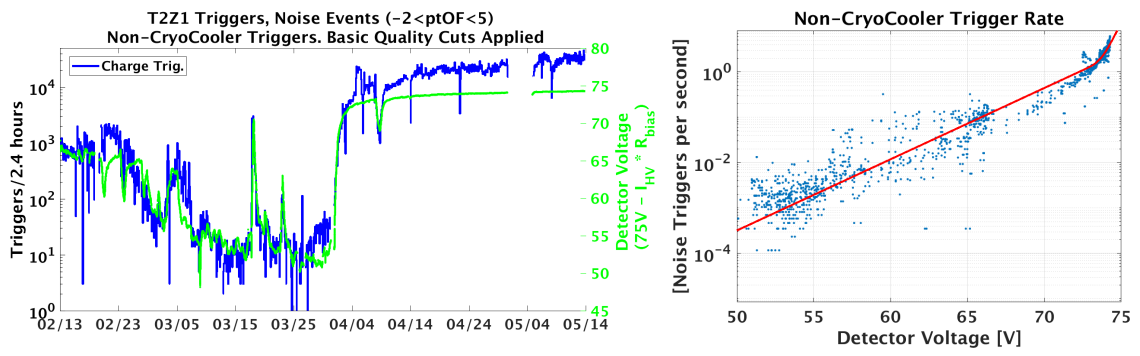

Figure 4.8: Correlation between trigger rate and detector voltage. The red curve is the best fit of the trigger rate to the detector voltage model of Eq. 4.2.

The detector bias voltage is the most important factor in CDMSlite's energy scale, so it is crucial to monitor the HV current in order to know the detector voltage over the course of the run. The value of $\mathrm{R}_{p}$ varies significantly over time, and Sec. 4.4.1 discusses how we correct the variable energy scale using the HV current readings.

\subsubsection{Charge Triggers}

The HV current readings have also led to an improved understanding of T2Z1's noise environment over time. Using the trigger rate as a metric for the noise environment (with a higher trigger rate corresponding to higher noise), Fig. 4.8 shows the dependence of the trigger rate on the detector voltage, after the majority of cryocooler-induced LFN triggers are removed. 
For $\left|V_{\text {det }}\right|<72 \mathrm{~V}$, there is an exponential dependence of the trigger rate on the detector voltage, and a sharply increasing power law dependence for $\left|V_{\text {det }}\right|>72 \mathrm{~V}$. The inability to fit all the data to one functional form suggests two things, the first trivial and the second more important and providing insights to the detector's HV behaviour:

1. Factors other than the detector voltage influence the charge trigger rate (e.g. May 09, etc.).

2. The process of charge trigger generation is different in different voltage regimes:

- Below $72 \mathrm{~V}$, there is no detector instability or "breakdown," and increase in the noise will be exponentially suppressed at low voltage.

- Above $72 \mathrm{~V}$, a "breakdown" process begins with a roughly powerlaw trigger rate dependence.

We fit for this voltage breakdown point, $\mathrm{V}_{0}$, with the functional form below:

$$
\text { TrigRate }[\mathrm{Hz}]= \begin{cases}B \times \exp [y \times V] & : V<V_{0} \\ B \times \exp [y \times V]+A \times\left(V-V_{0}\right)^{x} & : V \geq V_{0}\end{cases}
$$

and obtained a best fit value of $\mathrm{V}_{0}=72.3 \mathrm{~V}$.

Because of this dependence, when the detector voltage increased above $72 \mathrm{~V}$ (in magnitude) at the beginning of April, both the trigger rate and the observed noise became significantly worse. Two representative PSDs are shown in Fig. 4.9, where the left plot comes from a series in March and the right plot comes from a series in May. This figure shows that the noise from $0-1 \mathrm{kHz}$ (the signal band) increased by a factor of $\sim 3$ after $V_{\text {det }}$ increased above $72 \mathrm{~V}$, on April 1. In the Run 3 analysis, we consistently separate data according to these two distinct detector noise environments and refer to the two periods as R3a (which includes all series up to and including the April 1st series 1150401_1411) and R3b. Different detector resolutions and cut efficiencies are evident for the data obtained in R3a and R3b. 

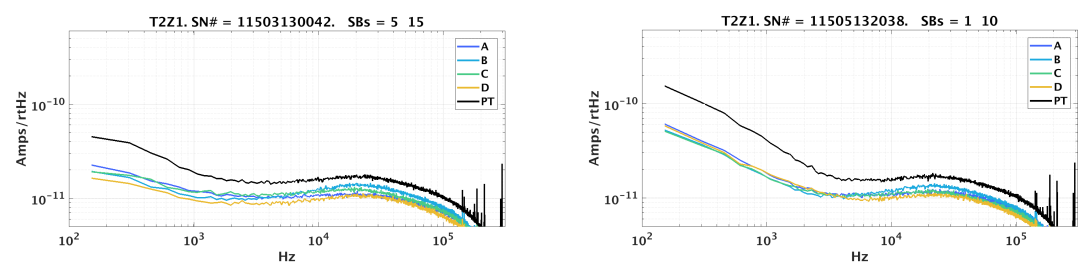

Figure 4.9: Representative PSDs from R3a (left) and R3b (right). The colored curves represent the noise on the individual channels, while the black curve represents the channel sum.

\subsection{Energy Scale}

\subsubsection{Energy Corrections}

As explained in Sec. 2.3, we estimate the amplitude of the total phonon pulse (the sum of the pulse from the 4 CDMSlite detector phonon channels) using the OF algorithms. Then, as explained in Sec. 2.5, we convert the OF amplitude to an energy using the ${ }^{71} \mathrm{Ge}$ EC events. However, even after applying this simple calibration factor, the OF-based energy estimate required correction for environmental and detector conditions.

In particular, we observed that the amplification of the detector drifted by up to $30 \%$ over the course of Run 3 . We found that we were able to correct for detector amplification drifts using known environmental variables. Once the corrections were applied, the resolution of the $K, L$, and $M$ shells were comparable to expected resolutions from the Run 2 analysis. The following subsections discuss the environmental variables and other inputs to the corrections.

\section{Current Correction}

Using the schematic in Fig. 2.16 we can solve for the detector voltage, relative to the applied HV voltage, using the measured HV current:

$$
V_{\mathrm{det}}=V_{b}-I_{\mathrm{HV}} R_{b} .
$$

The main assumption going into the model shown in Fig. 2.16 is that all the leakage current is downstream of the bias resistor, $R_{\mathrm{b}}$. We directly show that this is a reasonable assumption by verifying that the measured phonon energy of electron recoil calibration peaks depends on the detector voltage 


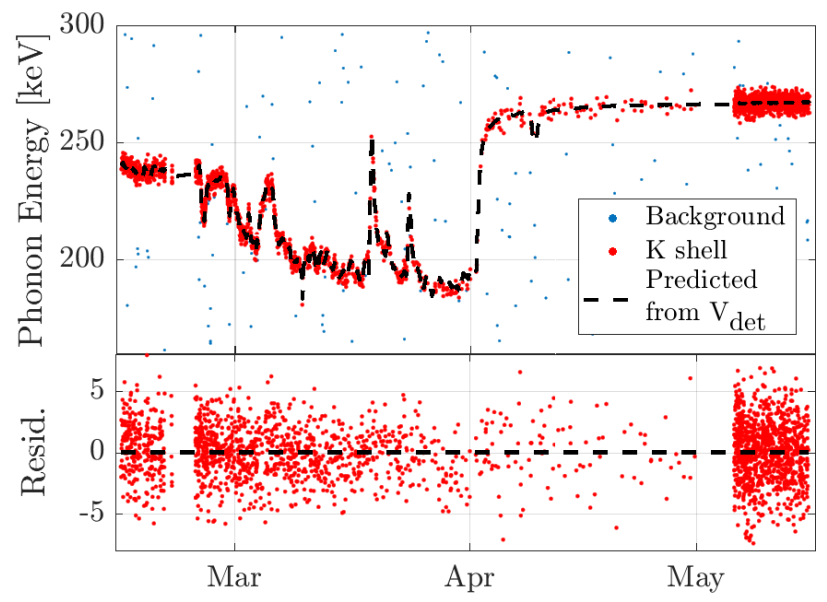

Figure 4.10: The drift in the total phonon energy of the $10 \mathrm{keV}$ line, with the HV current correction prediction overlaid. The other energy scale corrections have been applied to the data in order to highlight the corrections from the HV current.

as

$$
E_{\mathrm{t}}=E_{\mathrm{r}}\left(1+\frac{e V_{\mathrm{det}}}{\epsilon}\right)
$$

with $V_{\text {det }}$ defined in Eq. 4.3, and $E_{\mathrm{r}}$ is the recoil energy. When we solve for the expected total phonon energy of the $10 \mathrm{keV}$ calibration line using a $V_{\text {det }}$ that has been corrected by the measured leakage current $I_{\mathrm{HV}}$, we see good agreement with the drift of the $10 \mathrm{keV}$ line seen in the data, as shown in Fig. 4.10. The maximum current reached is $\sim 120 \mathrm{nA}$ which corresponds to an almost $30 \%$ correction. The HV current correction is significantly larger than the other energy corrections discussed below.

\section{Base Temperature Correction}

After the $10 \mathrm{keV}$ line has been corrected by the HV current, the measured energy shows a positive, roughly linear relationship with the cryostat base temperature. Unlike the HV current correction, the magnitude of this correction has not been determined from first principles. We fit this dependence to a straight line and then correct the energy variable in order to remove 


\subsection{Energy Scale}
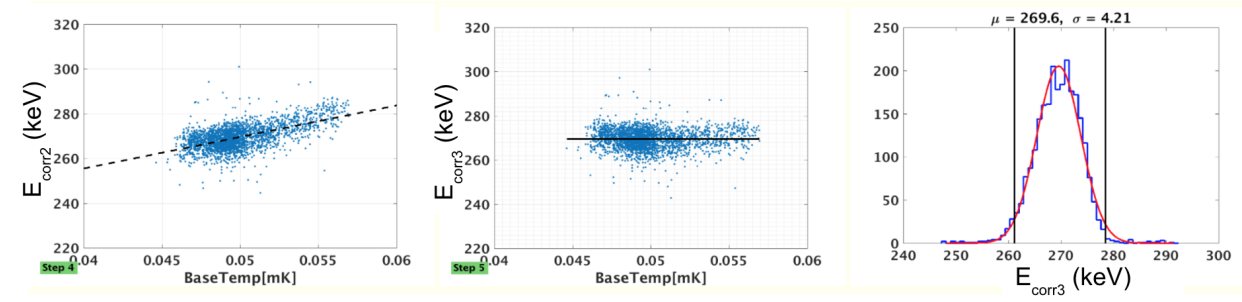

Figure 4.11: The steps of the linear base temperature correction. After fitting a line in the $E_{\text {corr } 2}$ (y axis) vs. base temperature (x axis) plane, the linear dependence is removed to form a corrected $E_{\text {corr } 3}$ variable. A Gaussian fit of the $K$-shell events' energies (as measured by $E_{c o r r} 3$ ) shows the energy resolution.

the dependence. The correction is given by

$$
E_{\text {corr3 }}\left(T_{\text {base }}\right)=E_{\text {corr } 2} \times\left(1+\left(T_{\text {base }}-\bar{T}_{\text {base }}\right) \times C_{\text {base }}\right),
$$

where $E_{\text {corr3 }}$ is the base temperature-corrected energy, $E_{\text {corr2 }}$ is the energy with just the $\mathrm{HV}$ current correction, $T_{\text {base }}$ is the base temperature, $\bar{T}_{\text {base }}$ is the mean base temperature over the course of the run, and $C_{\text {base }}$ is the slope of the linear fit. A visualization of the base temperature correction steps is shown in Fig. 4.11.

\section{Position Correction}

As discussed in Sec. 2.2.2, the position of the event in the detector affects the reconstructed energy of the event. This is referred to as "position dependence," and it is corrected for in a number of ways. The standard method employed by most iZIP detector analyses to reduce position dependence is to use the non-stationary optimal filter algorithm (Sec. 2.3), which deweights the beginning portion of the pulse where the pulse shape has the strongest dependence on the event's position. However, the non-stationary optimal filter best-fit amplitude is still found to contain some residual position dependence.

Figure 4.12 shows $10 \mathrm{keV}$ events from the T5Z2 detector operated in CDMSlite mode (single-sided readout) but at $0 \mathrm{~V}$. In this configuration, position-dependent effects are expected to be particularly prominent because there are no NTL phonons, which spread phonon energy out in a 

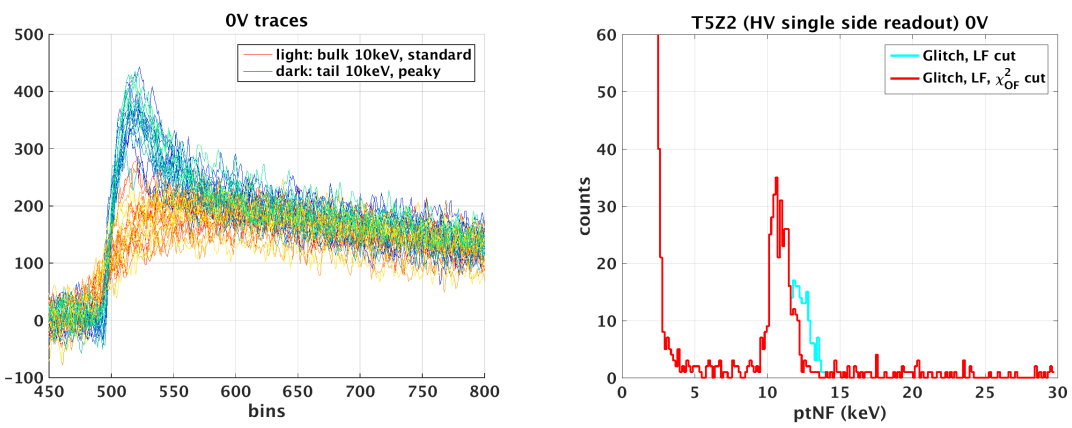

Figure 4.12: Data from single-sided readout of T5Z2 at 0V. (left) Raw total phonon traces plotted from $10 \mathrm{keV}$ events. These events all have the same energy (see alignment in the tail of the pulse), and the dark green-blue traces are selected by their peakiness. (right) The high energy $0 \mathrm{~V}$ spectrum asymmetry in the $10 \mathrm{keV}$ line, with the peakier $10 \mathrm{keV}$ events highlighted in cyan.

vertical column along the $e^{-} / h^{+}$track and therefore reduce position dependence. These data therefore serve well to demonstrate position-dependent effects: the peakier pulses (blue-green traces in Fig. 4.12 left) are reconstructed to a higher energy by the non-stationary optimal filter (cyan histogram in Fig. 4.12 right). Below we describe how we correct for the same position-dependent effects in the CDMSlite Run 3 data.

The two template (2T) fast amplitude measures the peakiness of the pulse and therefore is one measure of the position of the event. The variations in peaky vs. non-peaky pulse shapes, from the prompt phonons described in Sec. 2.4, correspond to the proximity of the original recoil to the phonon channels on the single side of the detector.

After the base temperature correction, as expected we see a positive linear relationship between the the non-stationary optimum filter amplitude and the 2-template fast amplitude. This correlation is shown in Fig. 4.13. We approximate this dependence as linear, fit a line to the data, and perform the following correction:

$$
E_{\text {corr } 4}\left(2 T_{\text {fast }}\right)=E_{\text {corr } 3} \times\left(1+\left(2 T_{\text {fast }}-2 \bar{T}_{\text {fast }}\right) \times C_{2 \text { Tfast }}\right)
$$

where $E_{\text {corr } 4}$ is the 2-template fast amplitude-corrected energy, $E_{\text {corr } 3}$ is the base temperature-corrected energy, $2 T_{\text {fast }}$ is the 2-template fast amplitude best fit, $2 \bar{T}_{\text {fast }}$ is the mean 2-template fast amplitude best fit, and $C_{2 \text { Tfast }}$ is 

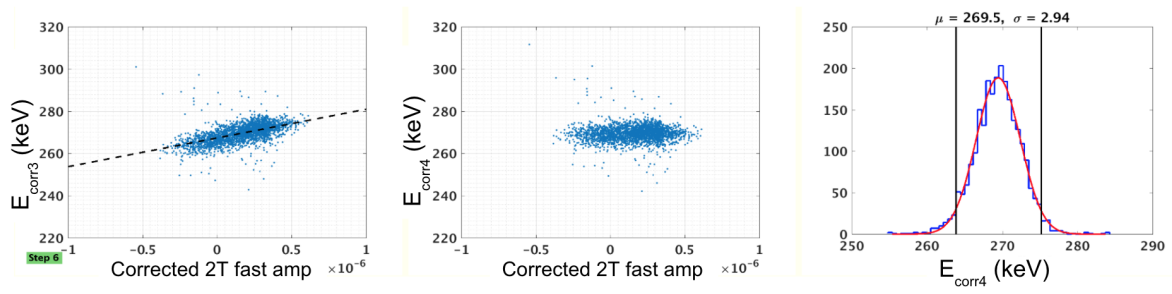

Figure 4.13: The steps of the linear 2-template correction. After fitting a line in the $E_{\text {corr } 3}$ (y axis) vs. 2-template fast amplitude (x axis) plane, the linear dependence is removed to form a corrected ptNF variable. A Gaussian fit of the $K$-shell in the corrected $E_{\text {corr } 4}$ variable shows improved energy resolution.

the slope of the linear fit. The mean energy of the $K$-shell events does not change with the correction, and the resolution improves from $4.21 \mathrm{keV}_{\mathrm{t}}$ to $2.94 \mathrm{keV}_{\mathrm{t}}$.

The corrections discussed above with respect to current and temperature are also applied to the fast amplitudes from the 2-template optimal filter fit. The fast amplitude can be positive or negative and does not have a direct correspondence to energy, and so the dependence of the fast amplitude for the $10 \mathrm{keV}$ events cannot be independently fit to variables (e.g. BaseTemp, $\mathrm{HV}$ current) in the same way as the slow amplitude. Instead it is assumed that the fast amplitude scales the same way as the slow amplitude, so we apply the corrections based on the fits to the slow template distributions. In Fig. 4.13, the "corrected 2-template fast amplitude" is corrected accordingly before it is used in the final correction for energy correction.

\subsubsection{Nuclear Recoil Energy Scale}

A subtlety in the energy scale corrections arises because we have applied the correction to all the data, but used ER events to derive the correction factors. While the base temperature and the 2-template fast amplitude corrections should not differ between ERs and NRs, the HV current correction does depend on the recoil type because it depends on the ionization yield. The energy scale has been corrected under the assumption that the ionization yield equals 1 . With a NR generating fewer free electrons, relative to an ER of an equivalent recoil energy, the NTL gain will be less for the NR and so the HV current/voltage correction factor will be smaller for the nuclear 


\subsection{Energy Scale}
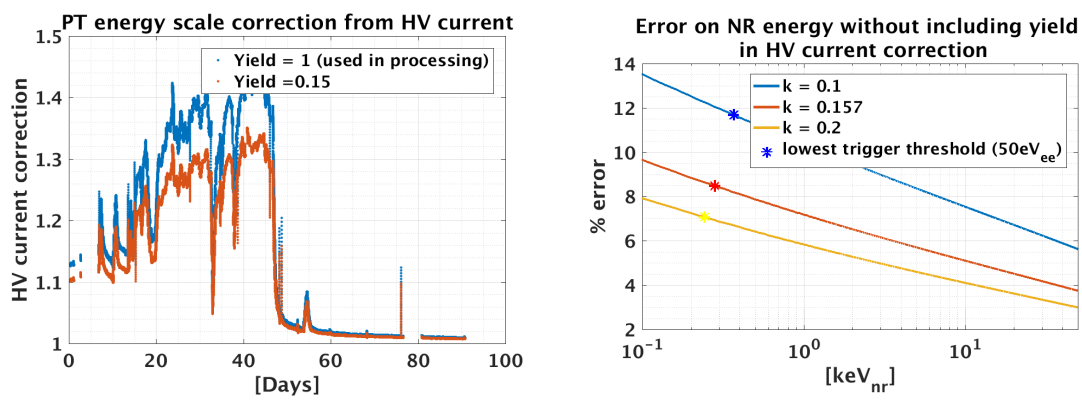

Figure 4.14: (left) Comparison of the correction factor for $Y=1$ and $Y=$ 0.15 events. Note that the difference in the correction factors is very small for small $I_{\mathrm{HV}}$, but for Run 3, with a leakage current up to $I_{\mathrm{HV}}=120 \mathrm{nA}$, it is clear that we should include this correction. (right) The maximum $\%$ error on a NR energy estimate $\left(E_{n r}\right)$, if the total phonon energy $\left(E_{p t}\right)$ is corrected without consideration of the ionization yield, which results in the nuclear recoil energy incorrectly set higher than what it would be when properly keeping track of the ionization yield in the HV current correction. The $k$ is the Lindhard $k$ parameter for the yield.

recoils.

First we obtain the ER HV current correction in Eq. 4.4, which converts a measured total phonon energy to a corrected total phonon energy:

$$
\left.E_{p t, c o r r}\right|_{e r}=\left[E_{p t, u n c o r r}\right] /\left[1-\frac{I_{H V} \times R_{b}}{\epsilon / e+V_{n o m}}\right] .
$$

The same calculation for nuclear recoils, now including the ionization yield $Y\left(E_{n r}\right)$, as described in Sec. 2.1.2, gives:

$$
\left.E_{p t, c o r r}\right|_{n r}=\left[E_{p t, u n c o r r}\right] /\left[1-\frac{Y\left(E_{n r}\right) \times I_{H V} \times R_{b}}{\epsilon / e+Y\left(E_{n r}\right) \times V_{n o m}}\right] .
$$

In Fig. 4.14 we estimate the maximum error (by using the maximum current $I_{H V}=120 \mathrm{nA}$ ) that would be introduced in the NR energy scale if we ignore the fact that our original HV correction assumed an ionization yield of one. This error is the ratio of Eq. 4.7 and Eq. 4.8:

$$
\frac{E_{n r, \text { ignoring yield in correction }}}{E_{n r, \text { including yield in correction }}}=\frac{1-\frac{Y\left(E_{n r}\right) \times I_{H V} \times R_{b}}{\epsilon / e+Y\left(E_{n r}\right) \times V_{n o m}}}{1-\frac{I_{H V} \times R_{b}}{\epsilon / e+V_{n o m}}} .
$$




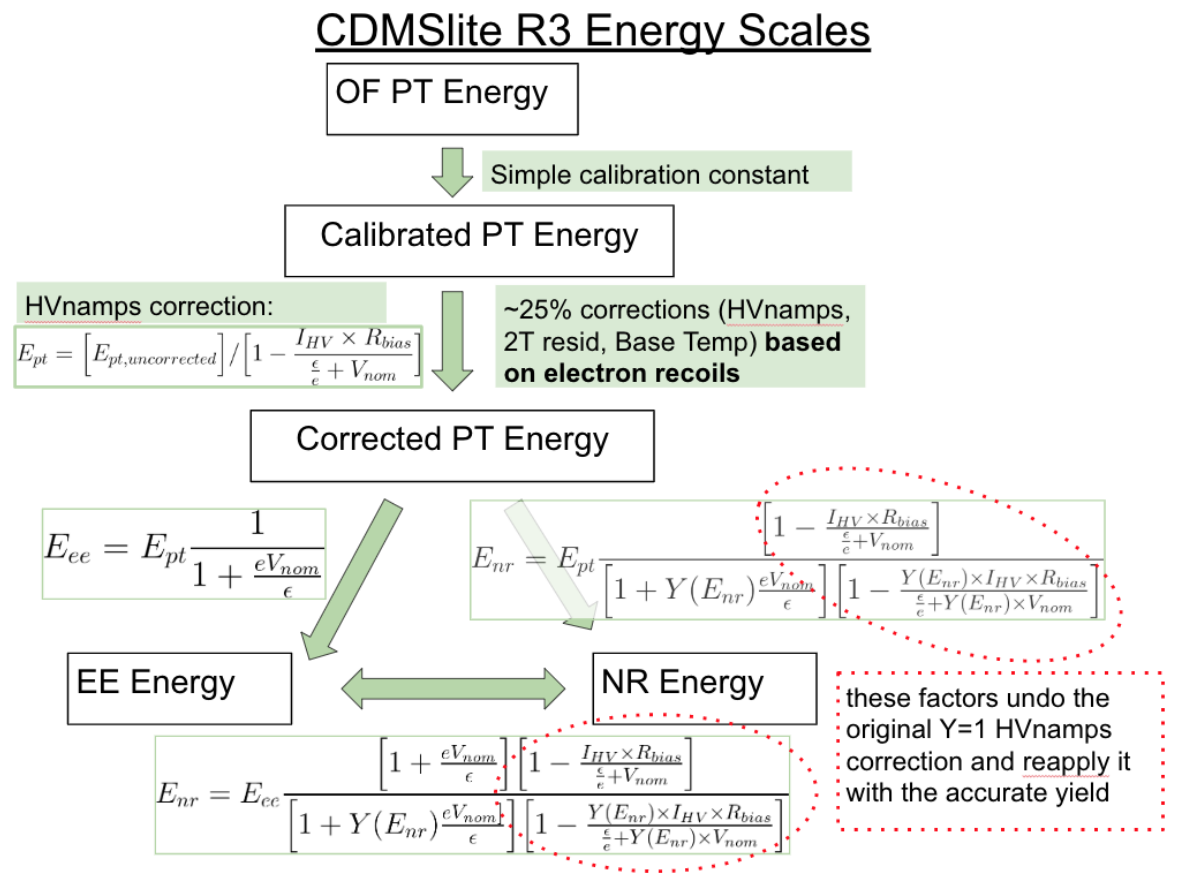

Figure 4.15: The energy scale conversions for Run 3. The differences from the Run 2 energy scalings, which are negligible only for small HV leakage current, are circled in red.

In order to account for this systematic error, when converting from corrected total phonon or electron equivalent energy to nuclear recoil energy, first the $Y=1 \mathrm{HV}$ current correction is undone, and then the correction is reapplied with the accurate yield based upon the event energy. The full energy calibration and correction flow chart is shown in Fig. 4.15.

\subsection{Quality Cuts}

\subsubsection{Overview}

As one of the first steps in our goal to look for a dark matter signal in the data, we must remove events that (1) were not caused by a particle interaction in the detector (which we refer to as "instrumental" events), (2) were likely to be reconstructed improperly by our event fitting algorithms, or (3) were recorded when the detector was behaving anomalously. In the process 
of removing these "bad events" with cuts, it is inevitable that some good events are also removed, and we account for this effect by computing the "signal efficiency" of these cuts. Throughout this section, we seek to define cuts that maximally reject the bad events but minimally reject good events, thereby maximizing the signal efficiency and increasing the DM sensitivity of the analysis.

Because this analysis employs profile likelihood methods to search for DM - fitting background and signal models to events that pass all cuts - it is imperative to identify and remove all instrumental noise events whose distributions cannot be modeled with a probability distribution in the fit. In the lowest energy range of the analysis, where the experiment is most sensitive to DM particles with mass $<10 \mathrm{GeV} / c^{2}$, the instrumental background is the highest. In this section I describe the different selection criteria to remove the more conspicuous types of instrumental noise events. In the next section, Sec. 4.6, I will describe the use of multivariate techniques to reduce instrumental noise leakage to less than 1 event while maintaining as low of an energy threshold as possible.

\subsubsection{Prepulse Noise Cut}

The raw data traces have been read out such that the $\operatorname{bin}^{8}$ that caused the trigger is the 500th bin of the trace (out of a total of 4096 bins), which is depicted in the example pulse in Fig. 2.5. The bins before this triggering bin are referred to as the "prepulse." Because the prepulse precedes the upward fluctuation that caused the trigger, it is useful for characterizing underlying "baseline" noise that exists in each channel. The standard deviation of the prepulse data points is recorded for every channel for every event. The distribution of the prepulse standard deviation of the randoms is fit to a Gaussian for each series (a roughly 3 hour period of time in which the SuperCDMS DAQ divides blocks of data), as shown for an example series in Fig. 4.16 left. Events whose prepulse standard deviation is outside the $4 \sigma$ range in any channel are removed by this cut, and the full Run 3 trend of prepulse baseline noise is shown in Fig. 4.16 right. Events that are removed have anomalously bad LF noise, electronic noise, or are pileup events. When applying the prepulse noise cut alone, the efficiency of this cut was found to be high, greater than $99 \%$ for nearly all series.

\footnotetext{
${ }^{8}$ The bin width is set by the digitization rate of the signal and is $0.8 \mu \mathrm{s}$, as previously provided in Table 2.3 .
} 

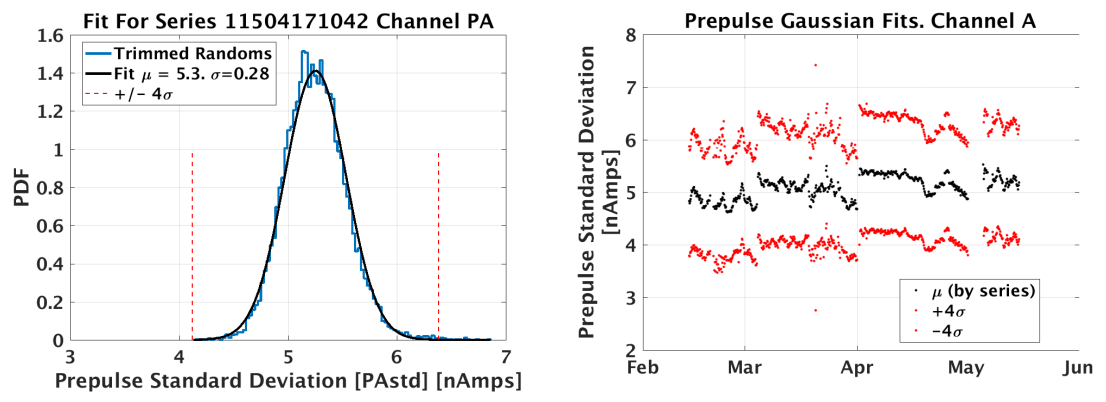

Figure 4.16: (left) The distribution of the prepulse standard deviation of the randoms for a single series, with a Gaussian fit to the distribution overlaid. (right) The trend of fitted Gaussian $\sigma$ for all Run 3 series.

\subsubsection{Bad Series Cut}

We remove series which had particularly poor noise performance or anomalous detector behavior with this cut. Shortly after the start of Run 3 there was a brief attempt to lower the hardware trigger threshold. Because this effort was unsuccessful (was met with high trigger rates) the lower threshold period only afflicted 6 series. These series are removed by this cut. Additionally, series with high trigger rates due to cold trap cleanings or immediately following detector calibrations were also removed. This cut removed only 11 series total, out of a total of 600 series. The livetime removed by this cut was less than $1 \%$ of the total Run 3 livetime.

\subsubsection{Phonon Pulse-Shape Cuts}

Information from pulse-shape fits can discriminate signal events from instrumental noise events having a characteristic pulse shape. Six different templates are fit to each event using the optimal filter method: a signal template, a square pulse template, an electronics glitch template with fast rise and falltimes, and three low-frequency noise (LF noise) templates. The signal, square pulse, and electronics glitch templates are shown in Fig. 4.17. The glitch and square templates were created iteratively in the following steps:

1. identifying, by eye, instrumental noise events in the data set

2. averaging the noise events to obtain a rough approximation of the instrumental noise events' shape 


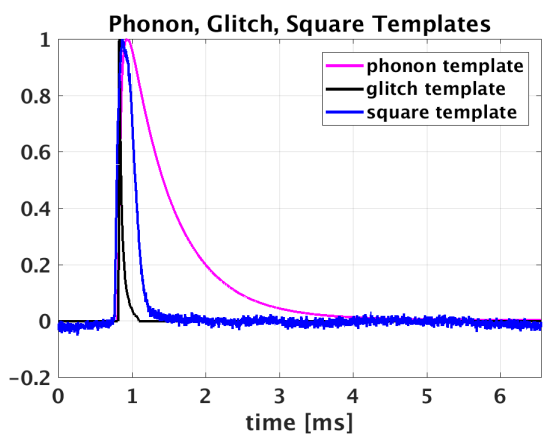

Figure 4.17: Comparison of glitch and square templates with the good phonon template. While the square template only roughly resembles a "square" shape, we refer to it as square because it is different from the glitch pulse primarily in that it plateaus for $300 \mu \mathrm{s}$, which it makes it wider.

3. processing the entire dataset with the rough templates

4. using $\chi^{2}$ information from the fits of the templates to the data, identifying all instrumental events that resemble the instrumental noise templates

5. normalizing and averaging all the instrumental noise events resembling either a glitch or square pulse into an improved (e.g. higher statistics) template

For the LF noise, three different templates were created because the LF noise assumes different pulse shapes, as discussed in Sec. 4.6.4.

The OF algorithm outputs a goodness of fit $\chi^{2}$ (that is smaller when the template is a good fit to the raw data trace), and therefore the difference of $\chi^{2}$, or $\Delta \chi^{2}$, between an OF fit with a "good event" template and an "instrumental" event template is a good indication of whether an event is an "instrumental" event. In fact, for consistent shapes of the instrumental noise pulses (i.e. pulse shapes that match the template) and Gaussian noise, this $\Delta \chi^{2}$ parameter is the optimal way to discriminate instrumental background from signal. The $\Delta \chi^{2}$ parameter is defined as:

$$
\Delta \chi_{\mathrm{LF}, \text { glitch,square }}^{2} \equiv \chi_{\mathrm{OF}}^{2}-\chi_{\mathrm{LF}, \text { glitch,square }}^{2},
$$

where OF corresponds to the standard signal-template fit, and LF, "glitch" and "square" correspond to the fits using the LF noise, glitch and square 

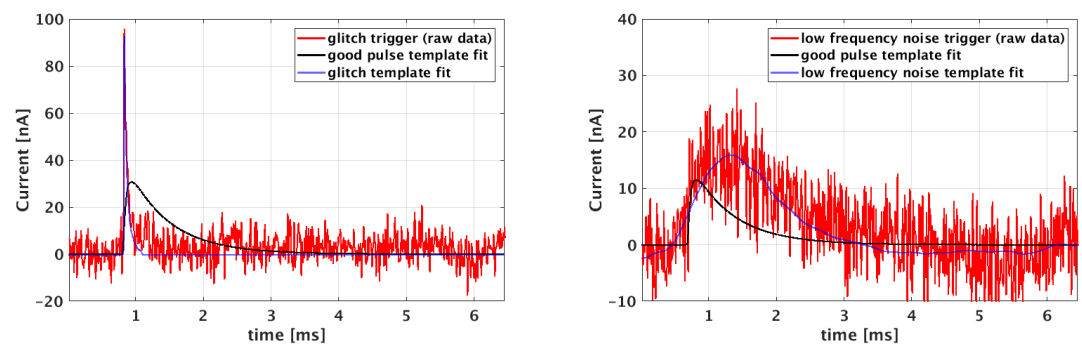

Figure 4.18: (left) An example of a glitch event with the best fits of the good event and glitch template to the event overlaid. (right) An example of a LF noise event with the best fits of the good event and LF noise template to the event overlaid.

pulse templates respectively. Lower values of $\Delta \chi^{2}$ indicate events that have a more signal-like shape.

Glitch events (for example the event shown in Fig. 4.18) and square events have relatively uniform pulse shapes and do not resemble the signal pulse shape. Therefore, a single template for each is sufficient to efficiently discriminate against these event types.

Every phonon chi-squared based cut boundary was defined not just based on the $\Delta \chi^{2}$ value, but also based on the events' reconstructed energy (i.e. ptOF). It is important to incorporate the event energy in the determination of the cut boundary because discrimination between signal and instrumental background is much more difficult at lower energy. The distribution of $\Delta \chi^{2}$ values for good events varies as a function of ptOF, and has a downward sloping parabolic shape, as shown in Fig. 4.19 left, so the cut boundary correspondingly takes on a parabolic shape in the ptOF vs. $\Delta \chi^{2}$ plane.

\section{Series Blocks}

Just as the distribution of $\Delta \chi^{2}$ values for good events changes as a function of ptOF, it also changes as a function of time. This motivates the division of the R3 data set into blocks of series, each of which having a custom cut boundary in the ptOF vs. $\Delta \chi^{2}$ planes

Initially it was not obvious why the ptOF vs $\Delta \chi^{2}$ distributions varied in time. The parabolas consist of good events that should not change pulse shape over the course of the run, so why does the parameter that fundamentally measures pulse shape (it depends on pulse shape based on $\chi^{2}$ values) vary in time for these good events? The origin of the variation is explored by 

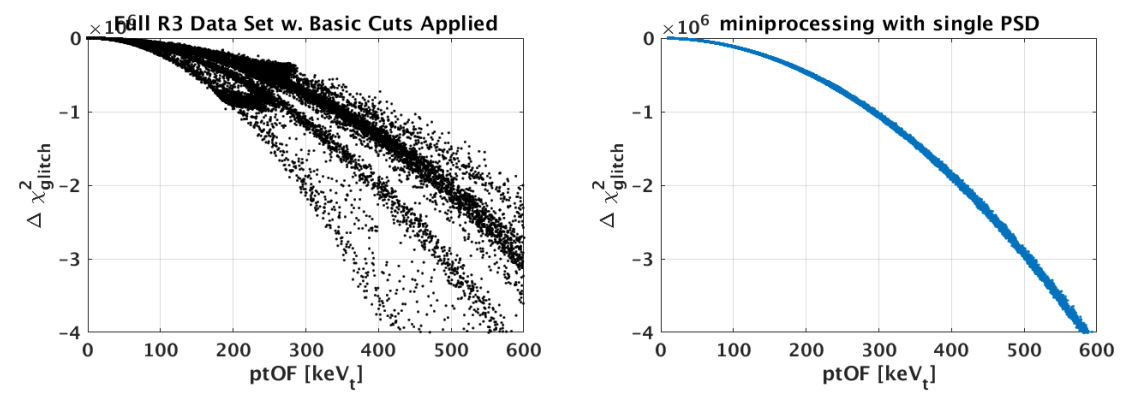

Figure 4.19: (left) The distribution of good events in the $\Delta \chi_{\text {glitch }}^{2}$ vs. ptOF plane, and (right) the same data reprocessed with a single noise PSD, showing that the variations are due to the PSDs that change for each series.

noting that, while the pulse shape should not change for these good events, the noise PSD that is used in the optimal filter fit changes on a series-byseries basis, and so we hypothesized that the time variations were due to the different PSDs used in the fit. The OF fits for each series are carried out with a noise PSD calculated from random events from that series. Figure 4.19 right shows the ptOF vs. $\Delta \chi_{\text {glitch }}^{2}$ plane for the same group of events as shown in 4.19 left, but processed with a single PSD; the single "good event" parabola in this plane shows that all of the variations are a result of the varying PSDs.

It still makes sense to use different PSDs for each series because incorporating the variable noise environment into the OF fits will obviously result in better fits. However, this deficiency in the data processing necessitated dividing up the Run 3 dataset into series blocks.

In addition to dividing the R3 dataset based on calendar time, it proved beneficial to divide the dataset based on the cryocooler vibration phase variable. Because this variable is correlated with the intensity of the low frequency noise environment on an event by event basis, data block divisions were created such that periods with particularly bad noise were isolated from the rest of the data set and harsher cuts could be defined for the noisier blocks.

To form the division boundaries, each series was first divided into a "loud" and "quiet" section based on the cryocooler vibration phase variable. Then, each series was characterized in three different ways:

1. The distribution of events in the ptOF vs. $\Delta \chi_{\text {glitch }}^{2}$ plane was fit to a parabola and the slope of the parabola was recorded. 


\subsection{Quality Cuts}
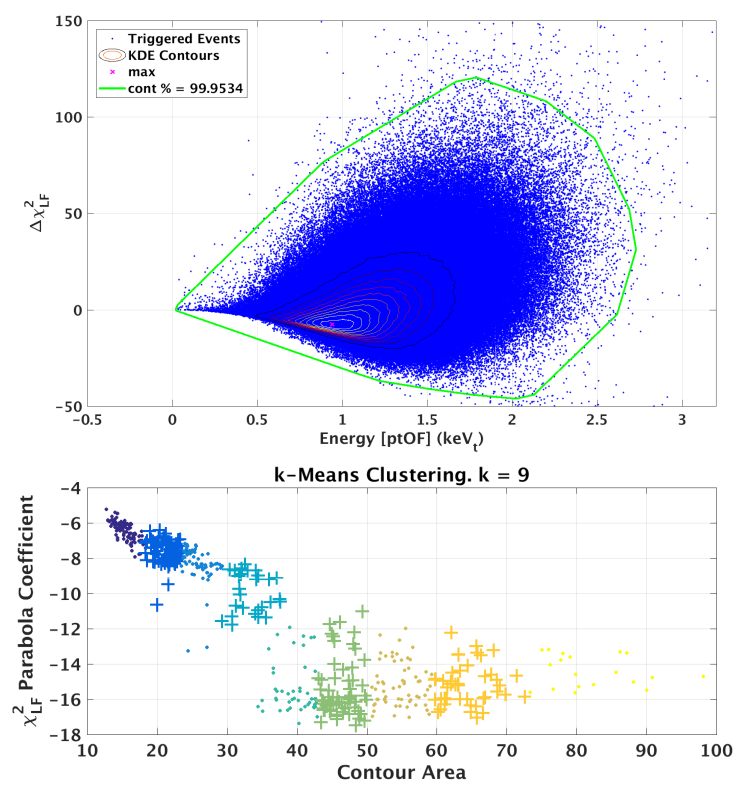

Figure 4.20: (top) The $\Delta \chi_{\mathrm{LF}}^{2}$ vs. ptOF plane for a portion of the Run 3 data, showing a high concentration of LF noise events. Raw pulse inspection revealed that only a small fraction of data points in this plot (those data point at the lowest $\Delta \chi_{\mathrm{LF}}^{2}$ values) resembled good events. The $\Delta \chi_{\mathrm{LF}}^{2}$ parameter is calculated with "template 1" as shown in Fig. 4.26. (bottom) The parabolic fit coefficient in the ptOF- $\Delta \chi_{\mathrm{LF}}^{2}$ plane vs. $3.5 \sigma$ contour area, with the clusters shown.

2. The distribution of events in the ptOF vs. $\Delta \chi_{\mathrm{LF}}^{2}$ plane was fit to a parabola and the slope of the parabola was recorded.

3. The $3.5 \sigma$ contour area of the LF noise events in the ptOF vs. $\Delta \chi_{\mathrm{LF}}^{2}$ plane (shown in green in Fig. 4.20 top) was recorded.

We designed a method for grouping series together according to the characteristic variables above, where the purpose of grouping series was to allow for the development of a cut boundary specifically for the series that well into that group. This method uses the k-mean clustering algorithm, which clusters series together that are close to eachother in the three dimensional space of parameters listed above.

The k-means clustering algorithm picks $\mathrm{k}$ centroids in an $\mathrm{n}$-dimensional space, assigns each data point to a centroid, and finds the distance between 
the points and the centroid looping over all centroids [103]. The program then iterates by moving around the centroids until the distance between the points and the centroid are minimized.

In deciding how many clusters to use, there is a tradeoff between many clusters (and capturing smaller changes in the distribution of events in the cut variables) and few clusters (and having good statistics with which to define the cut). For the cryocooler loud selection, with greater than 9 clusters the statistics become lacking in the cluster with the largest contour area. For the cryocooler quiet selection, using greater than 8 clusters only marginally shifts the divisions in the higher contour area clusters, and so 8 clusters are chosen for the crycooler quiet selection. The clustering groups are shown for the cryocooler loud series blocks in Fig. 4.20, where for this visualization the three dimensions of the clustering are projected into the $\Delta \chi_{\mathrm{LF}}^{2}$ vs. contour area $2 \mathrm{D}$ plane.

All the series blocks are also shown in Fig. 4.21 right. Figure 4.21 left shows events in the cryocooler vibrational phase fit to a 2 Gaussian + flat distribution. One Gaussian is fit around the region of largest event density (from $0-0.2$ seconds), and the $2 \sigma$ bound of the Gaussian is selected and included in the cryocooler "loud" category. If a second peak exists in the crycooler phase variable (which it does for a number of the series in April), a second Gaussian is fit to the second event density (from 0.3-0.4 seconds), and the $1 \sigma$ bound of the Gaussian is selected and included in the cryocooler "loud" category.

\section{Pulse Shape Glitch Events}

The origin of "glitch events" has been explained as electronic noise in the TES bias circuit that generates spiky pulse triggers with rise and falltimes faster than typical events, with time constants set by the TES response as opposed to phonon absorption times in the detector, as described in [104] (p. 197).

A cut boundary with two components was defined in the ptOF vs. $\Delta \chi_{\text {glitch }}^{2}$ plane for the 17 Run 3 series blocks. The first component was a flat cut as a function of energy and was defined using random triggers so that $>90 \%$ of randoms passed; this ensured that the cut has roughly $90 \%$ or greater signal efficiency at low energy. The second component was parabolic as a function of energy corresponding to the distribution of good events in the ptOF vs. $\Delta \chi_{\text {glitch }}^{2}$ plane. First a parabola was fit to all the triggered (i.e. non random) events in the series block, shown in Fig. 4.22 as the "central fit" parabola. Then the residuals of the $\Delta \chi_{\text {glitch }}^{2}$ values for the parabolic fit 

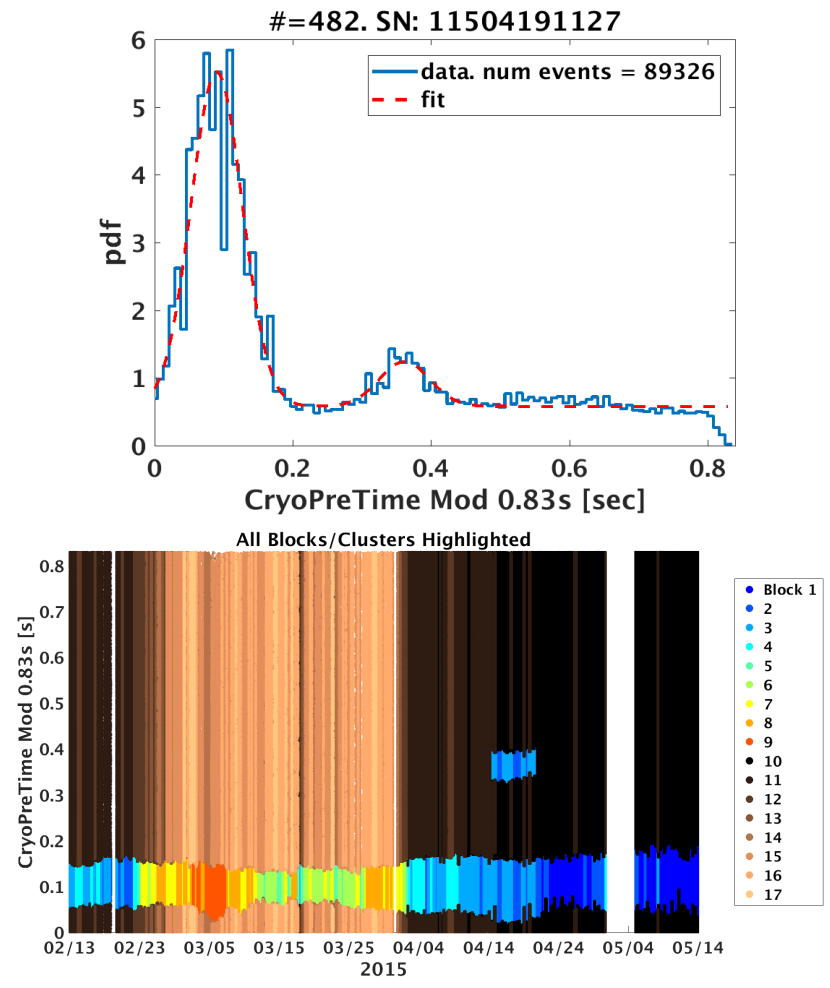

Figure 4.21: (top) The maximum likelihood estimate best fit of 2 Gaussians plus a flat distribution to noise events in a single series. (bottom) The 9 cryocooler "loud" clusters, and 8 cryocooler "quiet" cluster, all highlighted by time. 


\subsection{Quality Cuts}

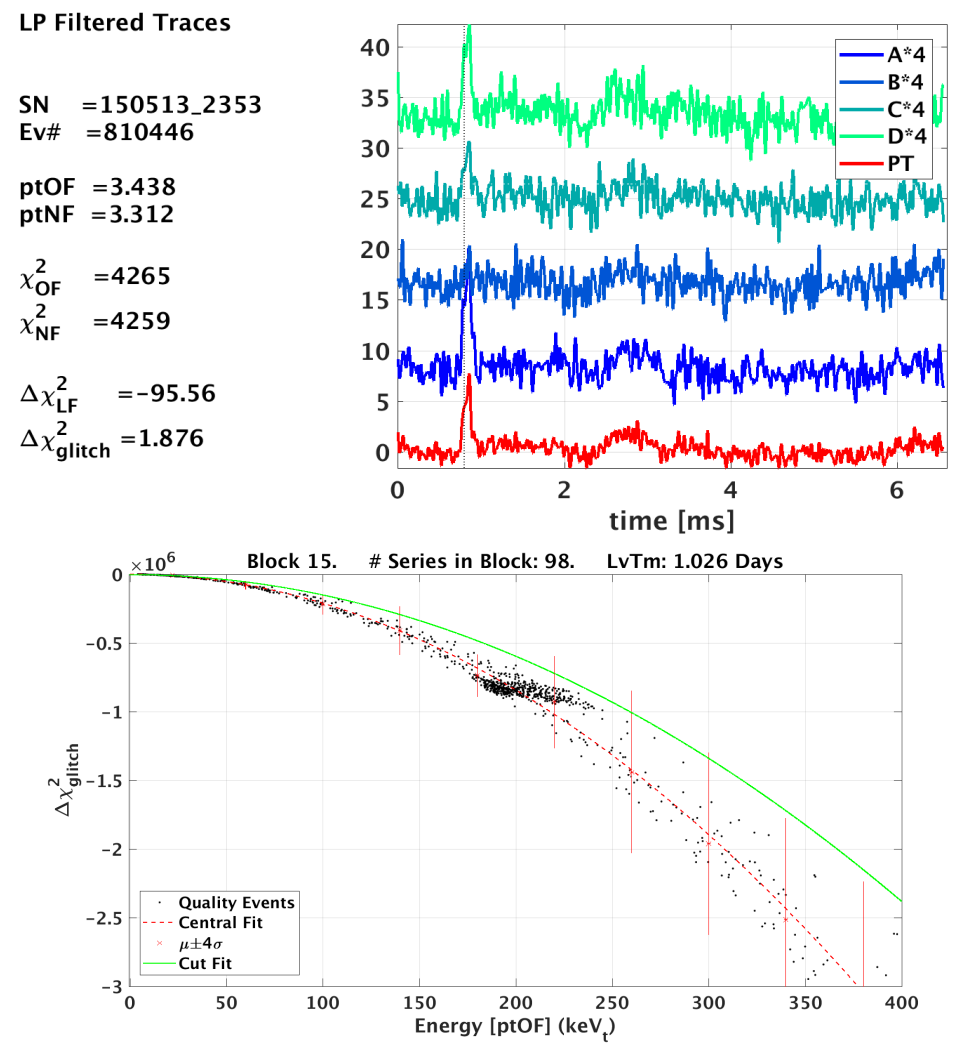

Figure 4.22: (top) A raw trace for a glitch-like event that fails the cut. (bottom) The tuning of the parabolic portion of the cut based upon the parabola of good events, for series block 15 .

and the data were binned by energy and a Gaussian was fit to the residuals, depicted as the red error bars in Fig. 4.22. Finally, a second parabola was fit to the $\mu+\mathrm{N} \sigma$ point of a Gaussian fit to events in each energy bin, where $\mathrm{N}$ was tuned for each of the 17 series blocks on the basis of raw trace examination. For series block 15 , we used $\mathrm{N}=4$ and the second parabola is given by the green "cut fit" line in figure 4.22, which defines the location of the cut. Fig. 4.22 left shows an example glitch event that is rejected by this cut. 


\subsection{Low Frequency Noise Cuts}
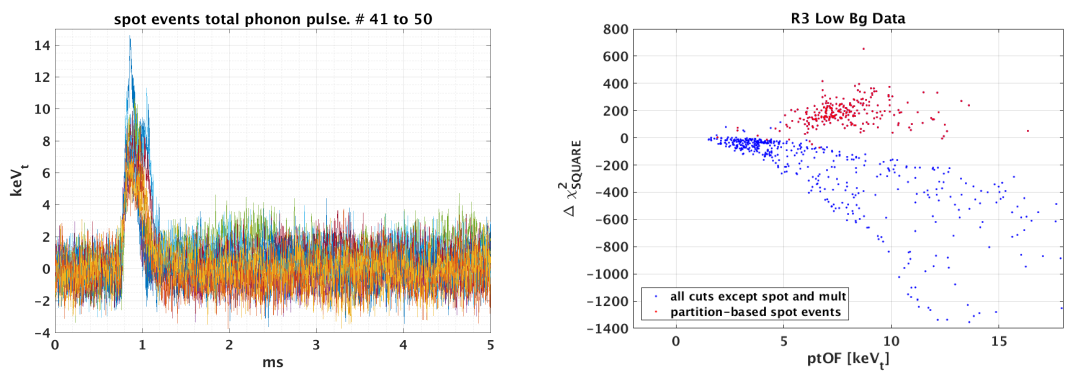

Figure 4.23: (left) The total phonon pulse of the square pulses. (right) the $\Delta \chi_{\text {square }}^{2}$ parameter formed from the square template.

\section{Square Pulse Cut}

This type of glitch has a fast rise and falltime, but its pulse shape is poorly approximated by the standard glitch template because the signal plateaus for $\sim 200 \mu \mathrm{s}$ at a maximum value before steeply falling. The total phonon traces from 10 example square pulse events are in Fig. 4.23. Because these events are not efficiently discriminated against with the glitch template, a custom template (which roughly resembles a square shape), was created to identify these events with a $\Delta \chi_{\text {square }}^{2}$ parameter.

When events are plotted in the ptOF vs. $\Delta \chi_{\text {square }}^{2}$ plane, Fig. 4.23 right shows a clear cluster of square events at high $\Delta \chi_{\text {square }}^{2}$ that are separated from the good event distribution, allowing for a highly efficient cut to be made in this plane. Note that in this plot, all data quality cuts other that the spot cut and multiples cut have been applied. A parabolic cut boundary was defined in the ptOF vs. $\Delta \chi_{\text {square }}^{2}$ plane for the 17 Run 3 series blocks.

\subsection{Low Frequency Noise Cuts}

\subsubsection{Motivation}

The majority $(>75 \%)$ of events in the Run 3 dataset are a result of low frequency noise fluctuations in the phonon signal that cause the detector to trigger. These events are particularly difficult to discriminate against for two reasons:

1. The dominant frequencies of good phonon pulses are relatively low $(<1.25 \mathrm{kHz})$ and so their bandwidth overlaps the LFN event bandwidth. The frequency band overlap can be seen clearly in the time 
domain in Fig. 4.18 right. This makes optimal filter pulse fitting less efficient for discrimination.

2. Unlike glitch and square events, the shape of LFN events is inconsistent and so a single LFN template does not produce a good fit for all LFN events.

These challenges become increasingly difficult to overcome as one tries to discriminate to lower energies, where the signal-to-(baseline) noise becomes low enough that the signal from an actual energy deposition and the fluctuation from a LFN feature are sufficiently buried under baseline noise that the small bump in the trace that caused the trigger could be attributed to either source.

Figure 4.24 demonstrates this challenge clearly: when the simulated good event signal is plotted on top of the data (which is dominated by LF noise triggers at low energy) in the ptOF vs $\Delta \chi_{\mathrm{LF}}^{2}$ plane, we see significant overlap of the two distributions. The overlap is especially bad in the energy region between the $50 \%$ trigger point (the lowest possible analysis threshold, discussed futher in Sec. 4.9.2) and $2.2 \mathrm{keV}_{\mathrm{t}}$. This is problematic because to set a cut with good signal passage near the $50 \%$ trigger point, we would also need to pass a large number of LF events. At the same time, a cut that removes the majority of the LF events has very low signal passage and effectively moves the analysis threshold above $2.2 \mathrm{keV}_{\mathrm{t}}$ because of its low efficiency. In the sections below, we describe the Run 3 campaign to remove this background from the dataset, which involved separate cuts for high and low energy events, as well as a multivariate boosted decision tree approach to improve discrimination in the low energy range.

\subsubsection{High Energy LF Noise Cut}

Section 4.6.1 described why LF noise discrimination becomes increasingly difficult at low energies. Therefore, at higher energies where discrimination is easier, a simple cut based on a $\Delta \chi_{\mathrm{LF}}^{2}$ parameter from a LFN template was designed to remove LFN that reconstructed above $5 \mathrm{keV}_{\mathrm{t}}$. This $5 \mathrm{keV}_{\mathrm{t}}$ value was determined so that a cut could be defined and have nearly $100 \%$ efficiency for good phonon events. The high energy LFN cut was defined individually for the 17 series blocks (following the same procedure as for the glitch and square pulse cut). 


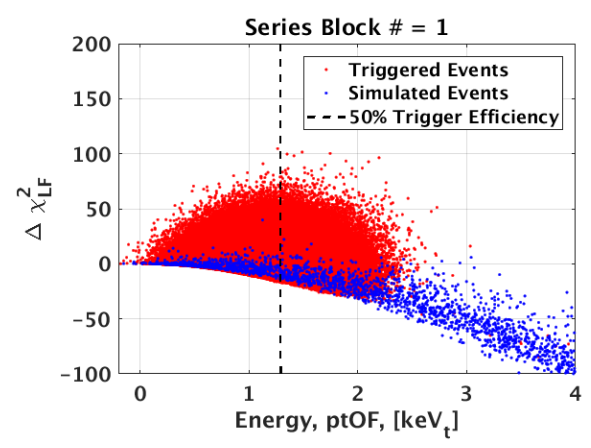

Figure 4.24: The simulated signal (blue data points) significantly overlaps the LF noise background (the majority of the red data points) at low energy in this variable plane.

\subsubsection{Detector-Detector Correlations}

To try to solve the signal-noise overlap problem shown in Fig. 4.24, we look for parameters other than the $\Delta \chi_{\mathrm{LF}}^{2}$ to efficiently identify and reject $\mathrm{LF}$ noise near threshold.

Because the LF noise originates from vibrations in the experimental setup, and because the vibrational sources producing LF noise triggers should couple to all detectors in a tower, we examined the correlations between the phonon waveforms on the CDMSlite detector and the other detectors in the tower.

In order to measure the correlation between raw total phonon traces, we use the Spearman correlation statistic [105], which is the same as the standard correlation statistic, except that the Spearman statistic ranks arrays (which in this application are the time domain traces) before calculating the correlation. "Ranking" is a simple transformation of an array where the value is replaced by the index of that value when the array is sorted from lowest to highest. For example, for the array $V=[4,8,9,1]$, the ranked array is $\operatorname{rg}_{V}=[2,3,4,1]$. Then the general definition of the Spearman correlation coefficient between arrays $\mathrm{X}$ and $\mathrm{Y}$ of length $N$ is

$$
r_{s}=\frac{\operatorname{cov}\left(\mathrm{rg}_{X}, \mathrm{rg}_{Y}\right)}{\sigma_{\operatorname{rg}_{X}} \sigma_{\operatorname{rg}_{Y}}}=\frac{\sum_{i}^{N}\left(\operatorname{rg}_{X, i}-\overline{\mathrm{rg}}_{X}\right)\left(\operatorname{rg}_{Y, i}-\overline{\mathrm{rg}}_{Y}\right)}{\sqrt{\sum_{i}^{N}\left(\operatorname{rg}_{X, i}-\overline{\mathrm{rg}}_{X}\right)^{2}} \sqrt{\sum_{i}^{N}\left(\mathrm{rg}_{Y, i}-\overline{\mathrm{rg}}_{Y}\right)^{2}}},
$$

where $\operatorname{rg}_{X}$ and $\operatorname{rg}_{Y}$ are rank variables. $\sigma_{\operatorname{rg}_{X}}$ is the standard deviation of the 

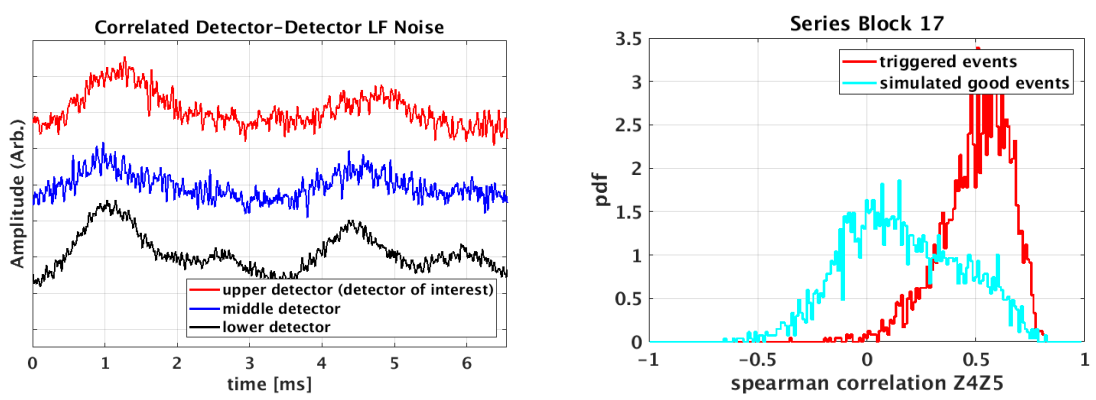

Figure 4.25: (left) Example traces showing detector correlated LF noise on the three Tower 2 detectors (top:T2Z1, middle: T2Z2, bottom: T2Z3). (right) Comparisons of signal and background distributions in the T2Z1T2Z2 detector trace correlation parameter.

ranked variables, and $\overline{\mathrm{rg}}_{X}$ is the average of the ranked variable. The Spearman correlation coefficient, as opposed to standard correlation coefficient, was used because it measures monotonic relationships in general as opposed to just linear relationships. Using the more general statistic was justified since we wouldn't expect purely linear detector-detector LF noise correlations. The correlation coefficient will be a number between -1 and +1 , with -1 being maximally anticorrelated, +1 being maximally correlated, and 0 being uncorrelated.

The Spearman correlation parameter between the time stream waveforms on the CDMSlite detector and the other detectors in the tower, for the events between the $50 \%$ trigger efficiency $\left(1.29 \mathrm{keV}_{\mathrm{t}}\right)$ and $3 \mathrm{keV}_{\mathrm{t}}$, is shown as the red histogram in Fig. 4.25 right. These events are in the region of signal-background overlap shown in Fig. 4.24, many of which are LF noise events,

In contrast to a LF noise trigger on T2Z1, a good event from a true energy deposition on T2Z1 should not be strongly correlated with the LF noise on the other detectors. We simulate the distribution of correlation coefficients for good events using the pulse simulation approach described in Sec. 4.9 for the pulses on T2Z1. On the other detectors, T2Z2 and T2Z3, we leave the randoms unchanged and calculate the correlation parameter between the simulated pulse on T2Z1 and the random noise event on the other two detectors. The cyan histogram in Fig. 4.25 shows the T2Z1T2Z2 correlations when the simulation uses randoms acquired at the end of the series. The event separation seen between the red (primarily LF 
noise) and cyan (signal) histograms is insufficient for setting an efficient cut in the correlation variable alone, but the separation between histograms indicates that the correlation parameters carry discrimination information. Therefore, as described in Sec. 4.6.5, we used the correlation parameters in a multivariate signal-noise separation boosted decision tree algorithm to improve the LF noise cut.

The names we use for the Spearman correlation variables are either "T2Z1-T2Z2 correlation" / "T2Z1-T2Z3 correlation" or, for brevity, "z4z5 correlation" / "z4z6 correlation".

\subsubsection{Motivation for Multiple Templates}

SuperCDMS Soudan iZIP analyses generated the instrumental noise templates [106], and were able to use a single template for each class of instrumental noise to remove instrumental backgrounds from the dataset. Because of the Run 3 analysis's goal to search for a DM signal to as low of an energy threshold as possible, and because of the particular importance of removing LF noise at low energies, we explored the use of multiple LF noise templates in order to improve the characterization of the LF noise that assumes different pulse shapes.

Development of new templates was also motivated because, when we average many low frequency noise total phonon traces on T2Z1 from the Run 3 dataset, the shape of the resulting average trace deviates from the low frequency noise template developed for the earlier SuperCDMS Soudan iZIP analyses. As shown in Fig. 4.26, the template developed in the iZIP analyses (magenta) does not have the $\sim 600 \mathrm{~Hz}$ oscillation that is seen in the average of many low frequency noise total phonon traces (black).

Figure 4.27 shows that the $\Delta \chi_{\mathrm{LF}}^{2}$ parameter based on the black template (template \#2) improves discrimination between signal and LFN background: with the same data selected, there is increased separation of the blue (signal) and red (background) data.

In addition, because the $600 \mathrm{~Hz}$ mode is seen so strongly in T2Z1 and the other Tower 2 detectors, as shown in Fig. 4.28, we test a discrimination parameter using the amplitude and $\Delta \chi_{\mathrm{LF}}^{2}$ fit values of a template isolated to this frequency. Averaging the Fourier transform of the individual LF noise traces shows a peak at $602.7 \mathrm{~Hz}$ with a phase of 114.6 degrees relative to the start of the trace. This phase offset is observed to be relatively constant, as expected, since the upward fluctuation of the noise causes the trigger. The resulting time-domain template is shown in Fig. 4.26 (left), labeled "template 3." 

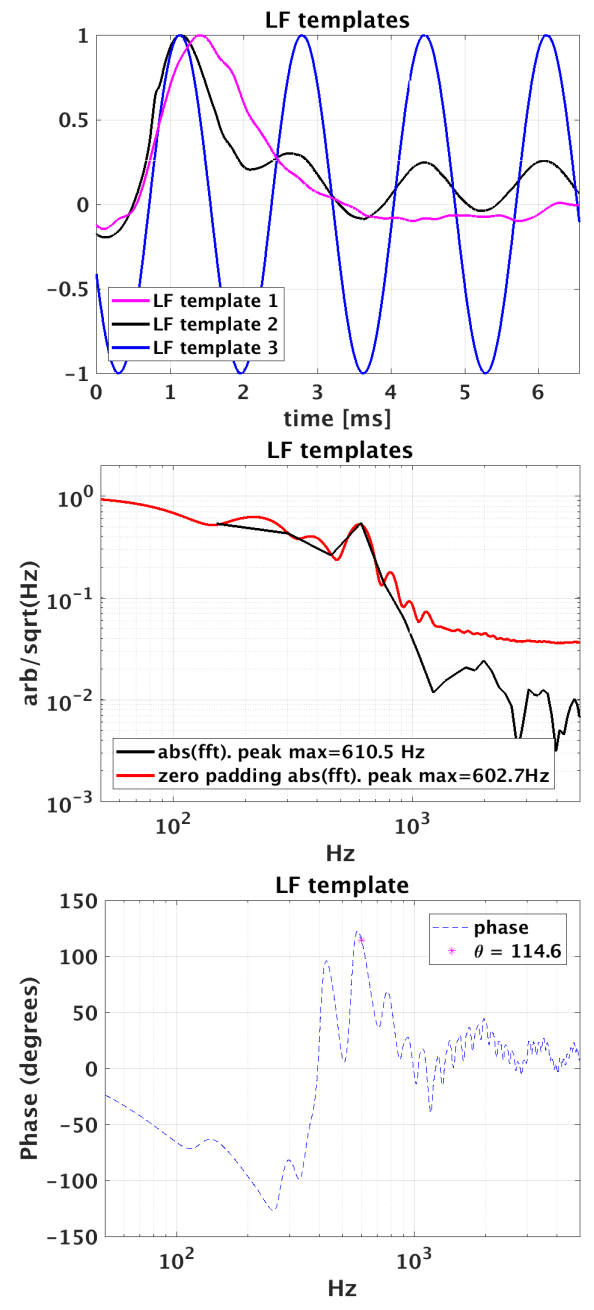

Figure 4.26: (top) Three different LFN templates used to fit different low frequency noise shapes on T2Z1. (middle) The averaged amplitude of the Fourier transform of the LFN traces. In the red curve, the time domain traces had zeros added to the front and end of the trace before the Fourier transform, which is a trick to to improve the $\delta f$ resolution of the Fourier transform. (bottom) The phase of the black LF noise template. 

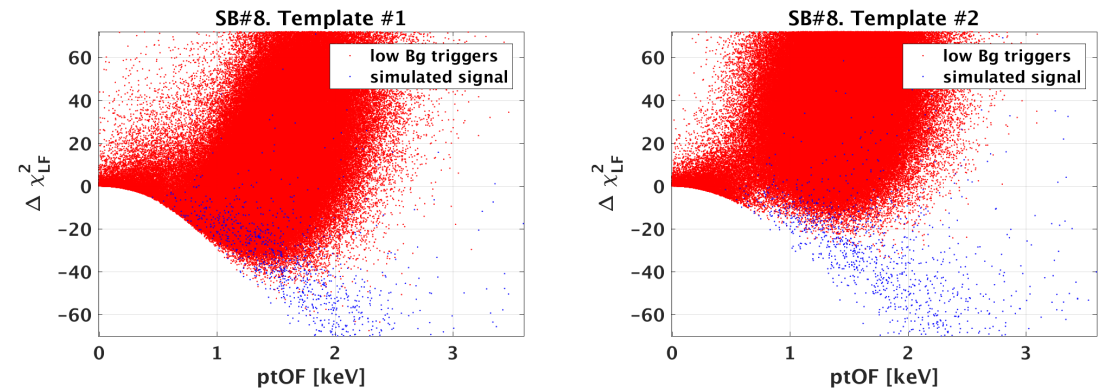

Figure 4.27: (left) The $\Delta \chi_{\mathrm{LF}}^{2}$ vs. ptOF plane for the data, shown in red, and the simulated signal, shown in blue, when using template \#1 to fit the raw traces. (right) equivalent to figure left but using template \#2, showing improved separation between the data (which consists primarily of LF noise events), and the signal.
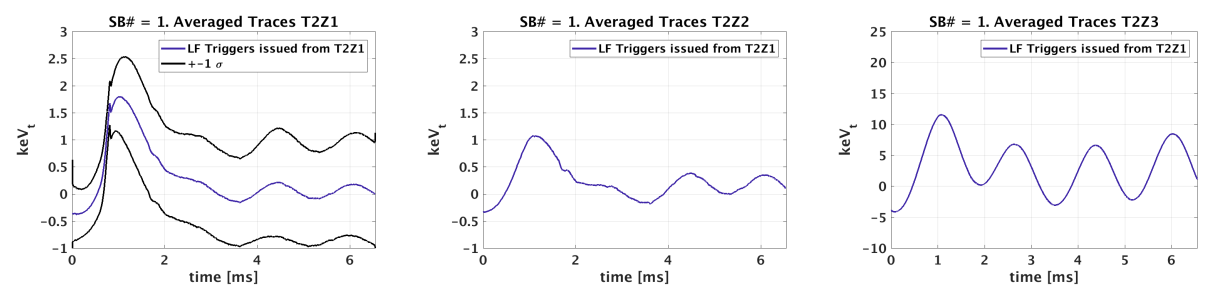

Figure 4.28: The average of LF noise events showing a coherent shape (since incoherent noise would average to roughly 0 on the y-axis) on T2Z1 (left) T2Z2 (center) and T2Z3 (right). 


\subsubsection{Boosted Decision Tree (BDT)}

A boosted decision tree is a well-suited algorithm to reduce the multiple LF noise parameters into a single BDT parameter (a score between -1 and +1 ) that maximizes the separation of signal from background. The primary "handles" on LF noise are:

1. the three $\Delta \chi_{\mathrm{LF}}^{2}$ parameters from pulse shape fits to the three different LF noise templates.

2. the $\hat{t}_{-}$variable, which represents the time since the last cryocooler cycle. The cycle period is $\sim 0.83$ seconds and LF noise causes triggers more frequently in the $\sim 0.2$ seconds after the start of the cycle, with the remainder of the cycle containing fewer LFN triggers.

3. the correlation of the phonon waveforms between the CDMSlite detector and the other detectors in the tower, because the vibrational sources producing LF noise triggers couple to all detectors in a tower.

More specifically, we use a BDT as a LF noise classifier to find a cut by reducing a multidimensional space down to a one dimensional space where signal and background are well separated. There are many options and different types of BDTs to use, and because only very small performance differences were observed when testing different BDT algorithms, we choose the Adaboost algorithm [107, 108]. The algorithm reduces overtraining using "ensemble methods," by forming many trees of low dimensions (2 or 3 ) and combining the results of these trees so that the classification of a single event depends on all the trees.

\section{Optimizer Fine Tuning}

In the two-level decision tree shown in Fig. 4.29, the bluer boxes are more signal-like, and the red boxes are more background-like. At every division of the tree, the population of events is divided based upon the variable that will maximally separate signal from background (computed with the gini index [109]). When using the Adaptive boosting method, at the lowest level of the tree, events that have been misclassified are increased in weight and supplied to additional small decision trees. The number of weak decision trees used in the algorithm is set by the analyzer, and should be determined by examining the error rate (the fraction of misclassified event) as a function of the number of decision trees. Once the training data sample has flattened on this plot, additional weak learners will not find additional correlations in 


\subsection{Low Frequency Noise Cuts}
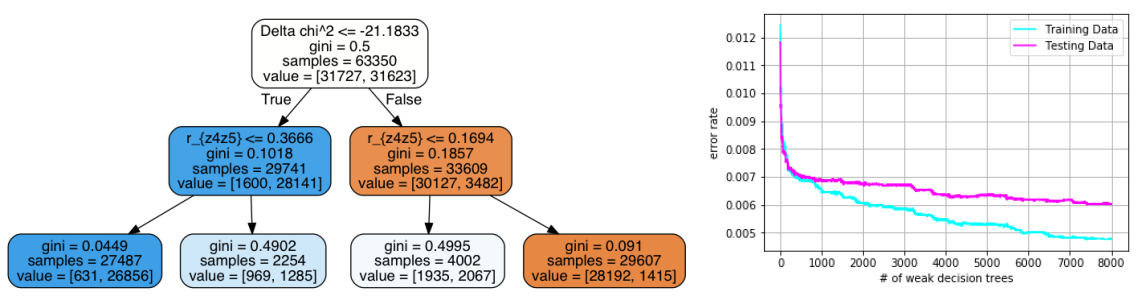

Figure 4.29: (left) A two-level decision tree. (right) A diagnostic plots of the error rate, for both training and test data, to help select the optimal number of weak decision trees.

the parameter space and therefore not improve discrimination. One strong indication of overtraining is that the test data curve increases. The number of weak decision trees was chosen to be 8000 , which ensured that the error rate for the test data did not begin to increase.

\section{Full BDT}

In addition to providing a powerful discrimination parameter, the BDT framework provides flexibility that allowed for avoidance of the series block data division procedure described in Sec. 4.7. While the series blocks based on calendar time and cryocooler vibrational phase were critical for the $2 \mathrm{D}$ ptOF vs. $\Delta \chi^{2}$ based cuts, for the BDT, instead of incorporating cryocooler information by dividing the data set according to cryo-loud and cryo-quiet time periods, it makes sense to to include the cryocooler score as a BDT input. Similarly, instead of dividing the data set according to calendar time, we can include the event time as a BDT input and the BDT will make a continuous cut based on the event time as opposed to our previous 17 discrete blocks approach.

At the same time, dividing the series up into time periods proved very useful when monitoring the detector's noise environment, and so below we consider the Run 3 data set in two different periods, divided into Run 3a and Run 3b as described in Sec. 4.3.4. The key reason for doing this is not because the BDT would be unable to find a LF noise correlation in calendar time and set the cuts accordingly. Instead, the reason for dividing the data along Run 3a and Run $3 \mathrm{~b}$ is because the nature of the background changes between the two periods. Whereas LF noise makes up the majority of low energy triggers in Run 3a, in Run 3b charge triggers and LF noise each make up roughly $50 \%$ of the low energy triggers, as shown in Sec. 4.3 .4 and 
in particular Fig. 4.6. It is simple to understand that BDT performance will be suboptimal if it is designed to discriminate against LF noise events, but the background is made up a combination of charge trigger events and LF noise events. We overcome this issue by training BDTs separately for Run $3 \mathrm{a}$ and Run $3 \mathrm{~b}$. For the Run $3 \mathrm{~b}$ data, the analysis threshold is raised from $1.29 \mathrm{keV}_{\mathrm{t}}$ to $1.9 \mathrm{keV}_{\mathrm{t}}$ in order to eliminate the charge leakage trigger background (which reconstructs to energies below $1.9 \mathrm{keV}_{\mathrm{t}}$ ).

Therefore, the full BDT score discriminator is based on 8 different parameters: (1) cryocooler phase (2) z4z5 correlation (3) z4z6 correlation (4)(5)(6) $\Delta \chi_{\mathrm{LF}}^{2}$ parameters for 3 different templates, (7) event time, and (8) energy, and the BDT is tuned for the two distinct time periods. The feature discrimination power of each of these parameters is shown in Fig. 4.30 for the Run $3 \mathrm{~b}$ BDT. It is clear that the detector-detector correlations and cryocooler information improves discrimination, even though the $\Delta \chi_{\mathrm{LF}}^{2}$ parameters provide the best discrimination.

Figure 4.30 right also shows the R3b BDT score vs. energy, where a population of good events is separated from the high density population of events made up of LFN triggers. What is also apparent, however, is that below $\sim 3 \mathrm{keV}_{\mathrm{t}}$ the distribution of events at higher BDT score begins to merge into the population at lower BDT score, again showing that discrimination is more difficult at low energy. It is not obvious from this plot where to place a cut on BDT score in order to maintain signal acceptance down to as low of an energy as possible, while simultaneously not accepting a LF noise event. In Sec. 4.7 below, we describe a procedure for splitting up the BDT score of Fig. 4.30 into two BDTs and setting a cut to ensure LF noise background leakage to less than one event.

\section{7 $\quad$ Bifurcated Analysis}

The bifurcated analysis is a method to measure the number of events from a certain background that is leaking past a set of quality cuts. The framework is suited for measuring leakage of a background that is difficult to model because neither a spectral energy shape (a PDF) of the background nor a background rate estimate is needed to employ it. The method estimates the leakage using side band information (i.e. information outside of the signal region) $[110,111]$.

Therefore, the bifurcated analysis is attractive when considering the Run 3 analysis goal to understand the background at the lowest energies where the dominant source of events is difficult-to-model LF noise triggers. 


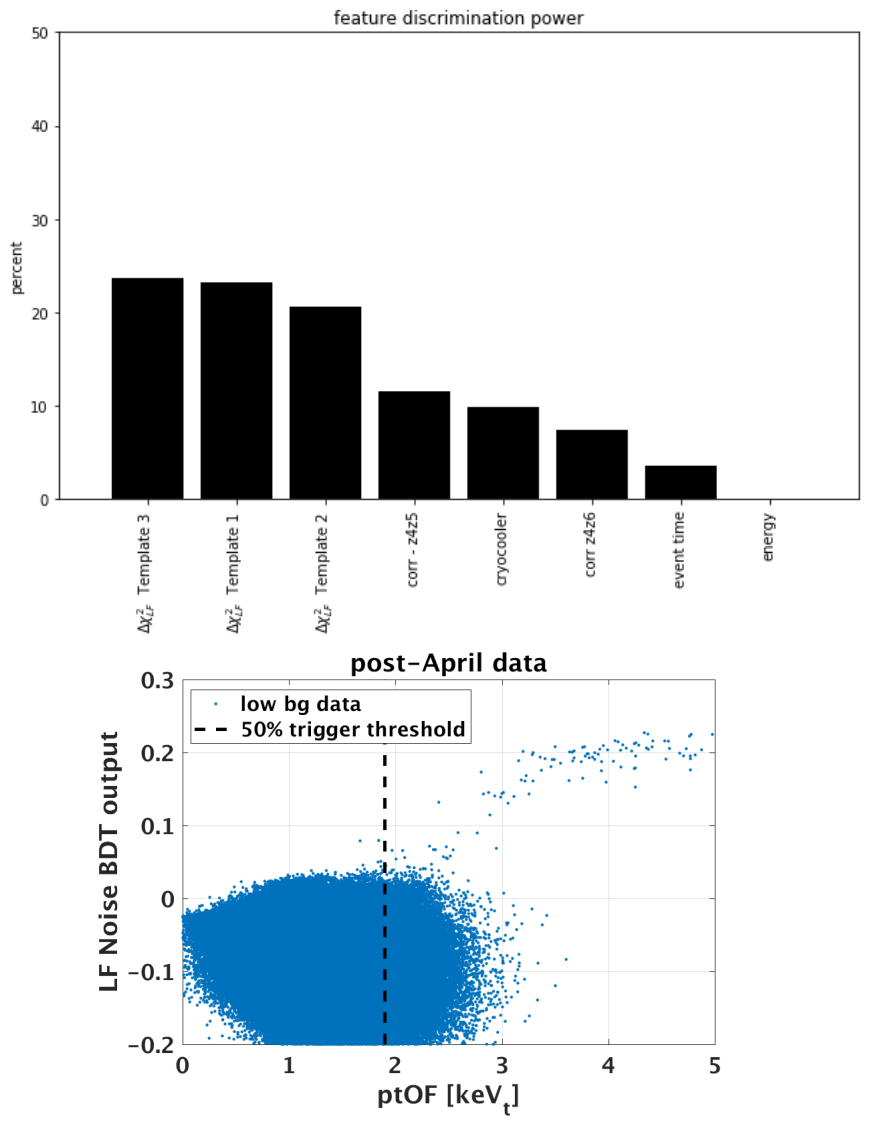

Figure 4.30: (top) The feature discrimination power of different BDT parameters, which is calculated using the amount that each feature split point improves the sample purity, weighted by the number of data points in the branch of interest, and summed over many decision branches. (bottom) The Run 3b BDT score as a function of energy, showing good separation of a small collection of signal events from the population of noise events . 


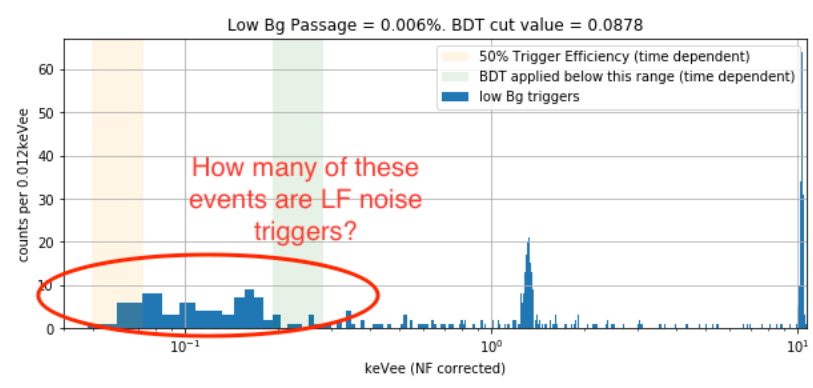

Figure 4.31: The CDMSlite R3 low background WIMP-search spectrum with a relatively loose cut placed on the LF noise BDT score. We seek to answer how many of the low energy events are LF noise triggers as a function of the cut value, and then set a cut such that there is $<1$ event leakage.

In particular, the LF noise cut must have a low ( $<1$ event) leakage of LF noise triggers past the cut. If LF noise trigger events leak in significant quantities past the cut, the background model will not describe the data. This is because the background model only includes components that describe particle energy depositions in the detector from radioactive decays. Figure 4.31 shows an example of an event spectrum with a relatively loose LF noise cut, where some LF noise events are present in the spectrum. If LF noise events leak past the final cut, they will be incorrectly incorporated into the PDF of a different background, and the likelihood fit for background and DM signal components will be systematically biased.

\subsubsection{Introduction}

The number of LF noise events leaking past a set of cuts is given by:

$$
N_{\text {leak }}=N_{\mathrm{LF}} \cdot P(\text { cuts }) \text {, }
$$

where $P$ (cuts) is the passage fraction of the cuts and $N_{\mathrm{LF}}$ is the number of LF noise events. While both $N_{\mathrm{LF}}$ and $P$ (cuts) are unknown, they can be estimated if there exist two uncorrelated sets of cuts that are both sensitive to LF noise events. The cuts are uncorrelated if cut A removes the same fraction of background regardless of whether cut B has been applied, and vice versa.

The two uncorrelated cuts that are both sensitive to the background of interest are labeled as cut $\mathrm{A}$ and $\mathrm{B}$. Then events are divided into the 


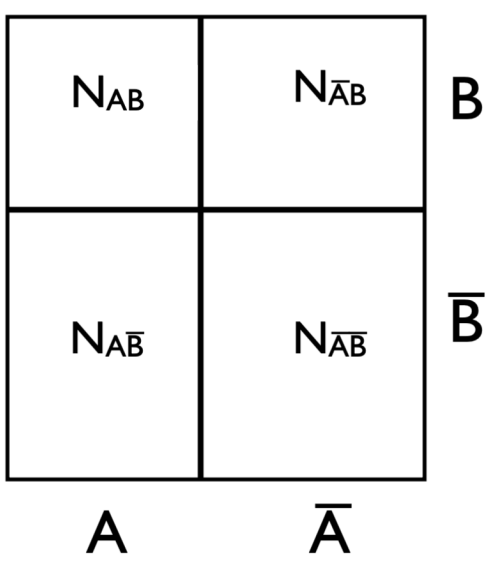

Figure 4.32: The 4 boxes into which events are divided when two bifurcated cuts (cut A and cut B) are applied to the data. The number of events in the upper left box $\left(N_{A B}\right)$ represents the number of events that pass both cuts.

4 different categories of passing and failing the cuts, which are shown in Fig. 4.32. The $A B$ box, which is the region of the parameters space that both cut $\mathrm{A}$ and cut $\mathrm{B}$ accept, is in the upper left. The number of events in the signal box is $N_{A B}$. Two types of events will be in the signal box: (1) good pulses from real energy depositions in the detector which we label $N_{S I G}$ and (2) low frequency noise triggers which we label $N_{L E A K}$.

In order to solve for $N_{L E A K}$, we must know the passage fraction of cut A and cut $\mathrm{B}$ to the background, which we label $P(A B)$. We also must know the signal efficiency ${ }^{9}$ of the two cuts, labeled $S(A B)$. Making the uncorrelated cut efficiency assumption, an assumption that is tested in Sec. 4.7.3 and 4.7.4, $P(A B)=P(A) P(B)$ and $S(A B)=S(A) S(B)$. We also define $N_{B K G}$ such that $N_{S I G}+N_{B K G}$ is the total number of events in all 4 boxes: $N_{S I G}+$ $N_{B K G}=N_{A B}+N_{\bar{A} B}+N_{A \bar{B}}+N_{\bar{A} \bar{B}}$. We also define $P(\bar{A})$ as the rejection fraction of the background (i.e. $P(\bar{A})=1-P(A)$ ), and use the equivalent notation for cut $B$ and for the signal efficiency $S$. The number of events in the individual boxes is therefore given by:

$$
N_{A B}=S(A) S(B) \times N_{S I G}+P(A) P(B) \times N_{B K G}
$$

\footnotetext{
${ }^{9}$ Throughout this section "signal" refers not necessarily to a DM signal, but rather any event that is not a LF noise background event.
} 


$$
\begin{gathered}
N_{A B}+N_{\bar{A} B}=S(B) \times N_{S I G}+P(B) \times N_{B K G} \\
N_{A B}+N_{A \bar{B}}=S(A) \times N_{S I G}+P(A) \times N_{B K G} \\
N_{\bar{A} \bar{B}}=S(\bar{A}) S(\bar{B}) \times N_{S I G}+P(\bar{A}) P(\bar{B}) \times N_{B K G}
\end{gathered}
$$

We numerically solve the 4 equations above for the 4 unknowns: $P(A)$, $P(B), N_{S I G}, N_{B K G}$. Then the number of background events leaking into the signal box can be solved for, and is given by:

$$
N_{L E A K}=P(A) P(B) \times N_{B K G} .
$$

If the bifurcated cuts are $100 \%$ efficient to signal the above system of equations can be analytically solved to give:

$$
N_{L E A K}=\frac{N_{A \bar{B}} N_{\bar{A} B}}{N_{\bar{A} \bar{B}}},
$$

but because we can determine the values of $S(A)$ and $S(B)$ from pulse simulations of good events, and they are $<100 \%$ efficiency, we numerically solve the system of equations and apply Eq. 4.17.

\subsubsection{Application to CDMSlite Run 3}

The BDT-based discrimination discussed in Sec. 4.6.5 is particularly well suited for the bifurcated analysis application. First, the bifurcated analysis is designed to work in two dimensions (i.e. the two dimensions on which the two cuts, A and B, can be placed), and a BDT reduces the dimensionality of the discrimination parameters from $\mathrm{N}$ inputs to a single output. Therefore, training two BDTs reduces the eight-dimensional discrimination parameter space to 2 dimensions that can be used for the bifurcated analysis.

Second, the bifurcated analysis is designed to work when the A and B cuts are uncorrelated. For the LF noise cut we have developed parameters from 3 different sources that all have LF noise discrimination power but are not necessarily correlated-(1) detector signal correlation information, (2) cryocooler information, and (3) pulse shape information with the $\Delta \chi_{\mathrm{LF}}^{2}$ parameters. To proceed with the bifurcated analysis we split up this information into two BDTs that each discriminate against LF noise but do so using parameters in which we would not expect there to be extreme correlations. 
The eight LF noise discrimination parameters are used in two different BDTs with the goal of creating BDT outputs that are good discriminators but uncorrelated. Branch $\mathrm{A}$ is primarily $\Delta \chi^{2}$ based and Branch $\mathrm{B}$ is primarily cryocooler and detector-detector correlation based, although the decision of which parameters to use in which branch was ultimately made empirically. That is, different arrangements of parameters were tested until an arrangement that yielded uncorrelated BDT outputs was found. For this reason the template $3 \Delta \chi_{\mathrm{LF}}^{2}$ parameter is not in the same branch as the other $\Delta \chi_{\mathrm{LF}}^{2}$ parameters, and the division of parameters is given below:

- branch A

$-\Delta \chi_{\mathrm{LF}^{-}}^{2}$ template 1

$-\Delta \chi_{\mathrm{LF}^{-}}^{2}$ template 2

- Event Time

- energy (ptOF)

- branch B

- cryocooler phase

- T2Z1-T2Z2 correlation

- T2Z1-T2Z3 correlation

$-\Delta \chi_{\mathrm{LF}^{-}}^{2}$ template 3

- Event Time

- energy (ptOF).

Figure 4.33 left shows the BDT output of the two different branches. There is a population of events in the upper right of the plot for which the output of the two BDTs are clearly correlated between the two BDTs. These are signal events and it is an indication that the two bifurcated BDTs are successfully discriminating; they are together separating events that the full BDT (indicated by the color) also separates with high BDT score. More importantly for the bifurcated analysis to work, the LF noise background (at lower BDT score) should be uncorrelated between the two cuts. The high density of events at significantly lower BDT score $(<0)$ is correlated between the two branches. This agrees with intuition, since very bad LF noise can be identified based on $\Delta \chi_{\mathrm{LF}}^{2}$ information as well as cryocooler or detector-detector correlation information. In order to apply the bifurcated analysis, additional cuts can be defined to select events closest to the cut 
boundary (where it's most important to measure event leakage) and as will be shown robustly below, the cuts are uncorrelated in the region of the BDT values where there is risk of LF noise events leaking past the cut.

\section{Setup Cut Verification}

In the bifurcated analysis framework, "setup" cuts are those that are applied to the data before performing the bifurcated analysis. In this case our setup cuts include all of the quality cuts described in Sec. 4.5, as well as a simple LF noise setup cut that removes particularly bad LF noise. The location of the setup cut, which is defined on the full BDT score, is shown in Fig. 4.33 center. This location was chosen to remove events that are sufficiently bad LF noise triggers that they can be taken out of the bifurcated analysis because they will not leak into the signal region. The events surviving the setup cuts - the events that will be used in the bifurcated analysis - are shown in black in Fig. 4.33 right.

By first looking at the population of events that fail the full BDT setup cut, shown in Fig. 4.34, we see that they are removed from the $A B$ box. However, because there are nearly $2 \times 10^{6}$ events that make up this population, we'd like to check that the tails of the distribution do not extend into the $A B$ box. A $2 \mathrm{D}$ kernel density estimate is used to approximate the distribution [112]. The bandwidth of the kernel - the width of the Gaussian that is added to each data point - is chosen automatically by the functions such that the density estimate is optimal for normal densities. When the KDE is found using this default bandwidth, the density is integrated over the signal region, and 0 events are found to be entering into the signal region.

\subsubsection{LF Noise Background Correlation}

Our full data set contains signal and background events. We would like to calculate correlation coefficients for the data from the background distribution only, but it is unknown which data points come from which distribution. However, we know the shape of the signal distribution, defined as $P_{S}$. We also can estimate the magnitude of signal and background data in the full data set, allowing us to define the distribution of all events as $P_{f u l l}=P_{B}+\frac{N_{S}}{N_{B}} P_{S}$. Using this information we can subtract off the effects of the signal data and calculate correlation coefficients for the background $P_{B}$ distribution alone.

To keep with standard convention for $2 \mathrm{D}$ correlations/covariances, we'll label branch A as X and branch B as Y. Using Pearson's correlation co- 


\subsection{Bifurcated Analysis}
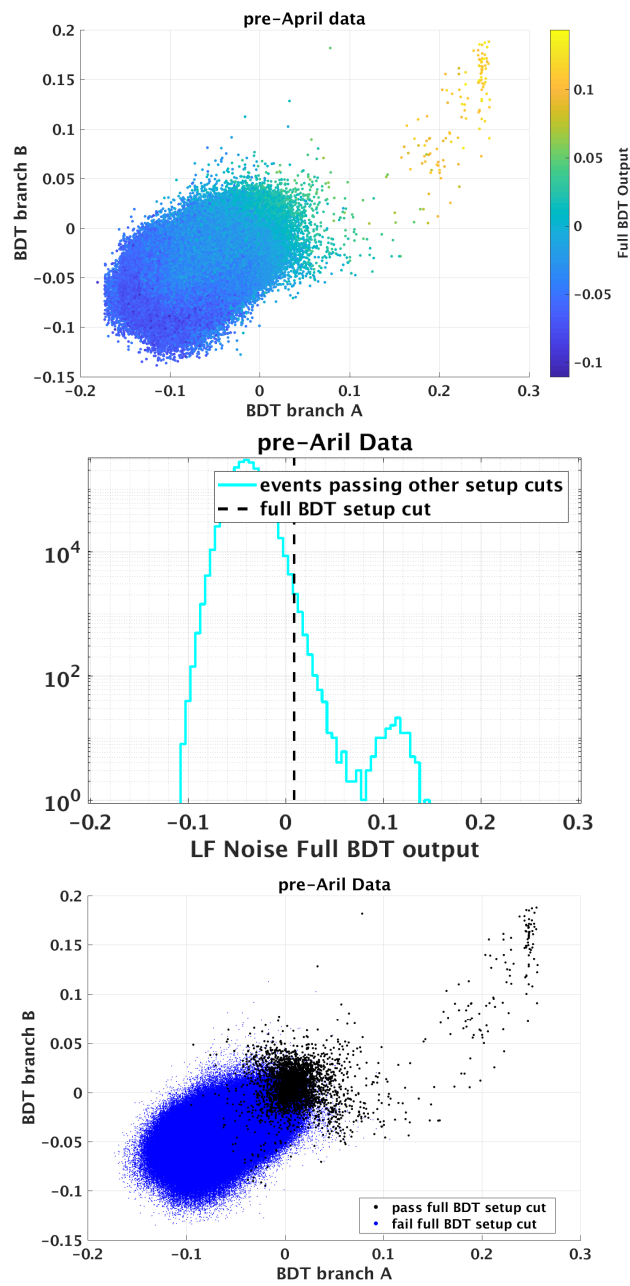

Figure 4.33: (top) The two branches of the bifurcated BDTs of Run 3a data, colored by the full BDT score. (middle) The setup cut value in the full BDT score. (bottom) The distribution of events considered for the bifurcated analysis shown in black, with those rejected by the setup cut shown in blue. 


\subsection{Bifurcated Analysis}

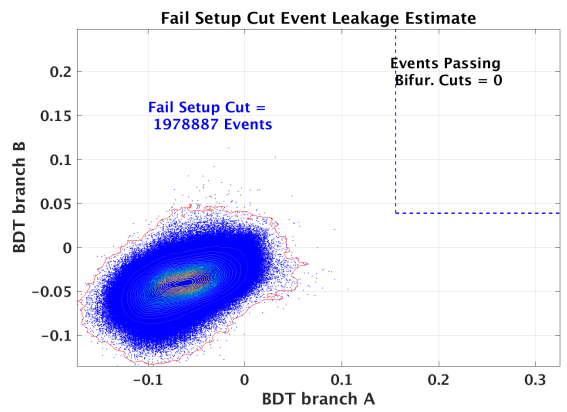

Figure 4.34: The distribution of events failing the setup cut, characterized with a kernel density. Integrating the kernel density into the signal region gives 0 events.

efficient, $\rho_{X, Y}=\frac{\operatorname{cov}(X, Y)}{\sigma_{X} \sigma_{Y}}$, we need to first calculate the covariance of the background distribution and we start with the signal data covariance:

$$
\operatorname{cov}_{S}(X, Y)=\iint d x d y\left(x-\left.\mu_{x}\right|_{B}\right)\left(y-\left.\mu_{y}\right|_{B}\right) P_{S}(x, y) .
$$

Equation 4.19 differs from the standard covariance equation only in that the $\left.\mu_{x}\right|_{B}$ and $\left.\mu_{y}\right|_{B}$ terms are the means of the background-only variables. The background distribution is unknown but these means can be estimated by using the full and signal means, $\mu_{\text {full }}$ and $\mu_{S}$, with the relationship:

$$
\mu_{f u l l}=\frac{N_{S}}{N_{B}+N_{S}} \mu_{S}+\frac{N_{B}}{N_{B}+N_{S}} \mu_{B}
$$

When going from continuous distributions to discrete data points, and calculating the sample covariance, it is important to normalize all the covariances to the estimated number of background events. With the sample covariance for the full data set given by

$$
\operatorname{cov}_{f u l l}(X, Y)=\frac{1}{N_{S}+N_{B}-1} \sum_{i}^{N_{S}+N_{B}}\left(x_{i}-\left.\mu_{x}\right|_{B}\right)\left(y_{i}-\left.\mu_{y}\right|_{B}\right)
$$

the background covariance is

$$
\operatorname{cov}_{B}(X, Y)=\frac{N_{S}+N_{B}-1}{N_{B}-1} \operatorname{cov}_{f u l l}(X, Y)-\frac{N_{S}}{N_{B}} \operatorname{cov}_{S}(X, Y) .
$$



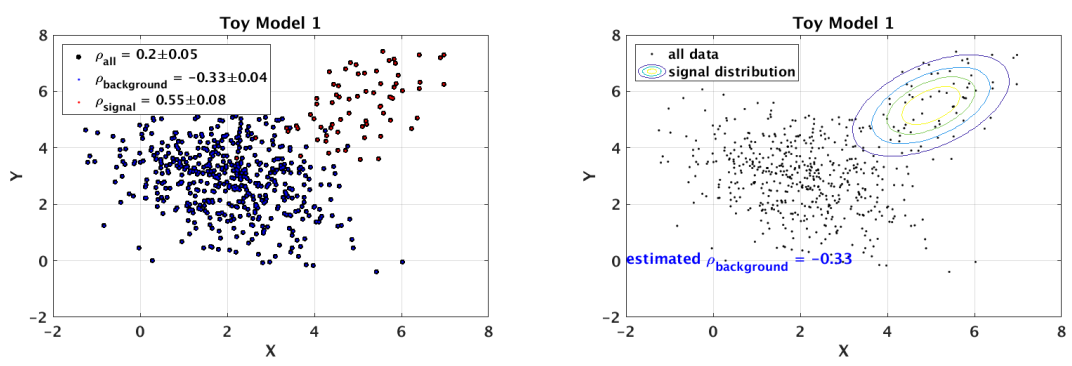

Figure 4.35: (left) A toy model of a background distribution (blue) and a signal distribution (red) with known correlation coefficient in the two variables. (right) The full signal + background distribution, where the correlation of the background distribution is estimated using the method described above, and agrees with the true correlation coefficient.

Once the variances for the background-only distributions are calculated using the same framework, the correlation coefficients between different variables of the background-only data can be calculated.

This method was tested on a toy model. The blue and red points in Fig. 4.36 left shows the two distinct distributions, where we seek to determine the correlation coefficient of the blue distribution. The true correlation coefficients are shown in the legend. The $\rho_{\text {background }}$ is the value that is being estimated using the information in the right figure. The full data set is shown in black and the "contamination" distribution is shown by the contours, and the relative amount of signal to background is known. The estimates of $\rho_{\text {background }}$ agrees with the answer given by the toy models.

This method was applied to the Run 3 DM search data as well as the simulated distribution of signal in the branch A and branch B BDT variable plane. The signal is simulated using the methods described in Sec. 4.9, and the distribution is shown in Fig. 4.36 left. We obtain a correlation coefficient of $\rho_{\text {background }}=-0.039$ for the LF noise background, as shown in Fig. 4.36. This correlation coefficient is relatively small, lending confidence to the requirement that the bifurcated cuts be minimally correlated, and the bifurcated analysis is performed with the $A B$ box defined as shown in Fig. 4.36, giving a LF noise leakage estimate of $0.3 \pm 0.1$ events. 

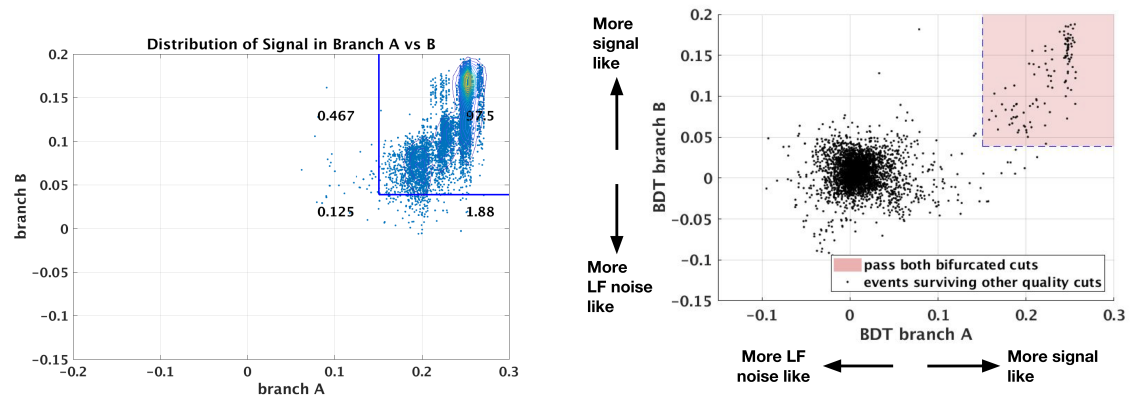

Figure 4.36: (left) The distribution of simulated signal in the bifurcated analysis cut plane. $97.5 \%$ of the simulated signal is contained in the passage box, while $0.47 \%$ is in the $A \bar{B}$ box, $1.9 \%$ is in the $\bar{A} B$ box, and $0.13 \%$ is in the $\bar{A} \bar{B}$ box. (right) The distribution of the DM search data in the bifurcated analysis cut plane. The points in the shaded pink area pass both bifurcated cuts.
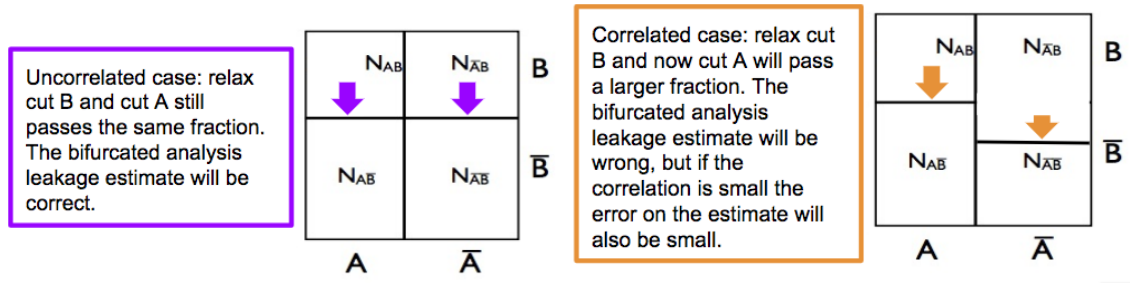

Figure 4.37: Examples of cut loosening when the cuts are uncorrelated (left) and correlated (right). 


\subsubsection{Box Relaxation}

While the correlation coefficient result above is an indication that the LF noise event distribution passing the setup cuts is uncorrelated, it does not ensure accuracy of the bifurcated analysis results. We must also check correlation of the bifurcated cut parameters directly in the vicinity of the cut location, and this is done with the box-relaxation method.

If the bifurcated result is accurate for a set of bifurcated cuts, then as a cut is loosened, new events will enter into the signal box and the new bifurcated leakage estimate will increase by the number of new events in the box (to within uncertainties). This procedure is complicated by the fact that the bifurcated analysis cuts are $<100 \%$ efficient to signal, as shown in Fig. 4.36 left. This means that as the box is loosened, we must account for the fact that an event that is absorbed into the signal box might be a signal event. Since the bifurcated analysis incorporates the signal efficiency, the $\mathrm{N}_{\text {leak }}$ estimate should not increase if the absorbed event is likely to be a signal event. We account for the $<100 \%$ signal efficiency by solving the non-linear equations of Eq. 4.13, 4.14, 4.15, 4.16, and 4.17.

After solving with a particular value of cut A and B, knowing the signal passage fractions and the total number of signal events $N_{S I G}$ allows one to calculate the estimated number of signal events outside the box, given by $(1-S(A) S(B)) \times N_{S I G}$. This value is shown in Fig. 4.38 center as a function of different values for the branch $B$ cut. In addition, as the branch $\mathrm{B}$ cut value is loosened the number of events in the $A B$ box increases, and this increase is shown in Fig. 4.38 left. To check the bifurcated analysis result, one would expect the number of new events in the $A B$ box minus the estimated number of new signal events in the $A B$ box (the difference between points in the left and center plot) to increase at the same rate as the bifurcated analysis's $N_{\text {leak }}$ estimate. In the right plot, this rate, which is called the "direct estimate" because it directly counts the data points, is overlaid on top of the bifurcated $N_{\text {leak }}$ estimate, showing agreement, to within uncertainty. We therefore verified that the number of events entering the box matched the bifurcated analysis's prediction to within uncertainties,

which is consistent with the cuts being uncorrelated and therefore supporting the validity of the leakage estimates.

\subsection{Fiducial Volume}

Because CDMSlite detectors have non-uniform electric fields, the NTL amplification (the second term on the right hand side of in Eq. 4.23) and the 


\subsection{Fiducial Volume}
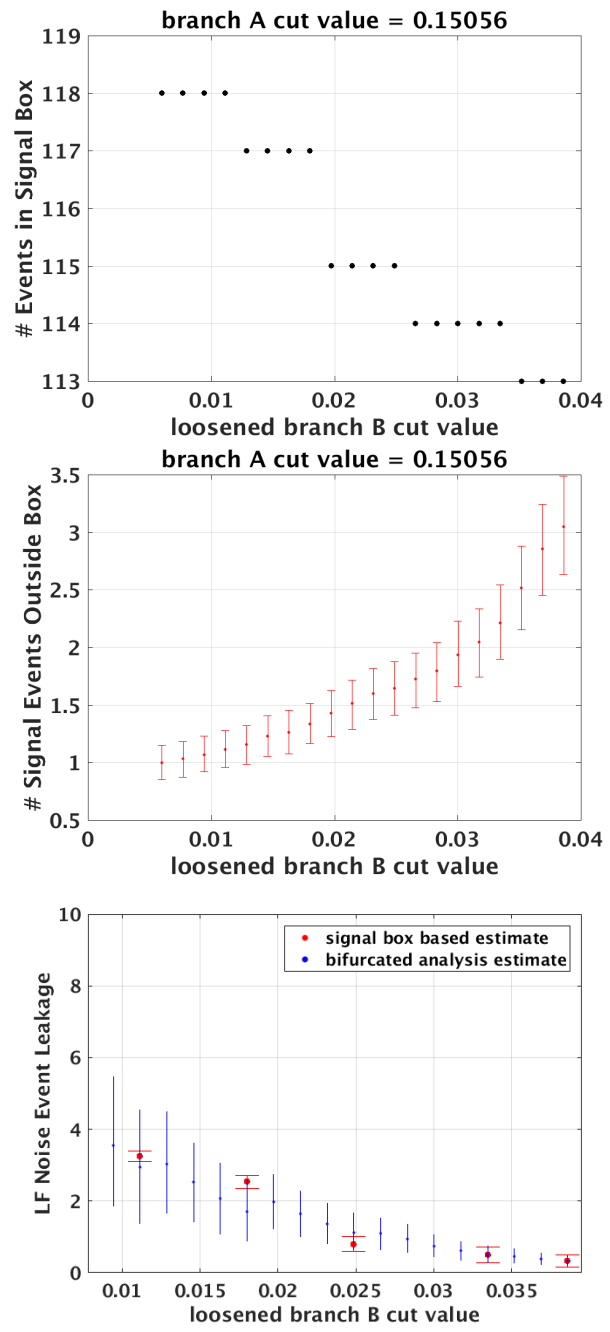

Figure 4.38: (top) The number of events in the $A B$ box, as the value of cut $\mathrm{B}$ is loosened. (middle) The estimated number of signal events outside the $A B$ box, as the value of cut $\mathrm{B}$ is loosened. (bottom) The number of new events in the $A B$ box minus the number of estimated signal events shown in red, as well as the bifurcated analysis's leakage estimate in blue. 

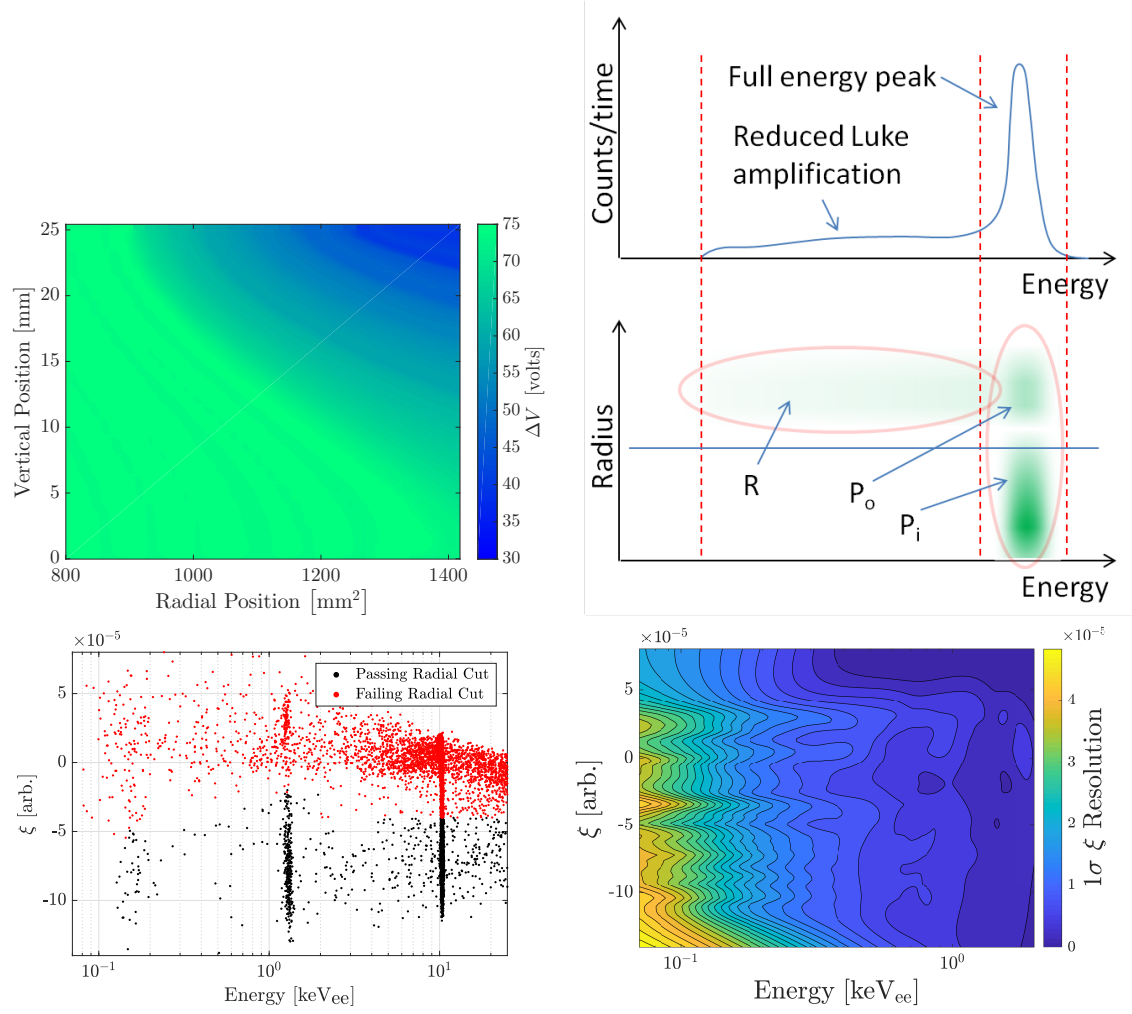

Figure 4.39: (top left) Calculated voltage map for high radius events, showing the difference in electric potential $\Delta V$ between the final collection points of the positive and negative charge carriers, as a function of initial position of the pair (plotted as radius squared vs. vertical position). Here, the top of the crystal is biased at $75 \mathrm{~V}$ and the bottom is grounded. Charge carriers in the outermost (radius $>800 \mathrm{~mm}^{2}$ ) detector annulus can experience less than the full detector bias voltage (credit: D. Barker [113]). (top right) A cartoon of the radial vs. energy distribution for a homogenous and monoenergetic background in the CDMSlite detectors. The $P_{i}$ and $P_{O}$ refer to inner and outer probabilities, and these variable names are used in the radial cut efficiency calculation of Sec. 4.9.5 (credit: M. Pepin [82]). (bottom left) Distribution of the radial parameter $\xi$ vs. energy in the DM search data. An energy-dependent cut on $\xi$ defines the fiducial volume below $2 \mathrm{keV}_{\text {ee }}$, while a stricter constant cut is used above $2 \mathrm{keV}_{\text {ee }}$. (bottom right) Resolution $(1 \sigma)$ for $\xi$ (radial parameter) shown as a function of $\xi$ and energy. At lower energy, the resolution worsens as the increased noise affects the reconstruction of the radial parameter. (credit: R. Underwood [114]) 
reconstructed recoil energy vary with the location at which an event takes place inside the detector:

$$
E_{\mathrm{t}}=E_{\mathrm{r}}+E_{\mathrm{r}} \frac{e \Delta V}{\epsilon} .
$$

For most events, $\Delta V$ in Eq. 4.23 is equal to the full potential difference between the detector faces, resulting in maximal NTL amplification. However, as shown in Fig. 4.39, near the detector sidewall $\Delta V$ can be smaller. The voltage drop experienced by an electron-hole pair, and thus the NTL amplification, can be reduced such that the reconstructed energy of some high-radius events is significantly lower.

The reduced NTL events are a particularly undesirable background since their energy is improperly reconstructed. Figure 4.39 top right shows an example of this background: for monoenergetic and homogeneously distributed source of events, for example the $K-, L-$, and $M$ - shell peaks, the electric field non-uniformity creates a low energy "tail" at the energies below the peak, as shown in Fig. 4.39 (top right). We therefore design a parameter that uses pulse shape information to estimate the radial position of an event in the detector. Then we place a cut on the this parameter to remove the reduced Luke gain events from the dataset, as shown in the cartoon in the top right.

The radial parameter for CDMSlite $\mathrm{R} 3$, which we refer to as $\xi$, is constructed using individual channel fit parameters from the two-template Optimal Filter Phonon algorithm (2TOF), exploiting the layout of the channel map and in particular the outer annulus of channel A (see Fig. 4.4). The parameter was developed by W. Rau [91] for CDMSlite Run 2, and we repeated his steps to calculate the parameter for Run 3 [115]. To first order, the two-template radial parameter (2TRP) takes the difference in the amplitude of the fast template for channel A with the fast template of the inner channel with the largest amplitude. This can be thought of qualitatively as creating a parameter that is correlated with the peakiness of the pulse on channel A relative to the other inner channels.

\subsubsection{Radial Pulse Simulation}

In order to remove reduced NTL events effectively, we must understand how these events are distributed in the radial estimator $\xi$ vs. energy plane. In particular, the resolution of $\xi$ degrades at lower energies because the lower signal-to-noise ratio of the pulses inhibits the ability of the 2TOF to extract position information. Fig. 4.39 bottom left shows the "flaring" of the 
radial parameter at lower energies, which represents its worse resolution at lower energy. Below $2 \mathrm{keV}_{\text {ee }}$ we model the $\xi$ vs. energy plane using a pulse simulation similar to that described in Sec. 4.9.3. Events in the $L$-shell (1.21-1.45 $\left.\mathrm{keV}_{\mathrm{ee}}\right)$ which pass all other quality cuts are used as model events, as they span the full volume. After the $L$-shell events are fit using the twotemplate Optimal Filter (2TOF) algorithm, we take the 2TOF amplitudes and re-scaled them to a lower energy. These templates are then added to a noise trace from end-of-run randoms from the same series as the event. For a constant input radius, the output radius is found to be Gaussian distributed in $\xi$, and the width of the Gaussian is shown in Fig. 4.39 bottom right. We use the radial resolution in the $\xi$ vs. energy plane as one step in the modeling of the reduced NTL events.

The radial resolution map shown in Fig. 4.39 can be thought of as part of the detector's radial response function. For inputs to this response function, we will estimate the true distribution of reduced NTL events in the $\xi$ vs. energy plane and convolve this distribution with the radial resolution response.

\subsubsection{Energy Distribution of Reduced NTL events}

For the energy distribution of reduced NTL events, we use a smoothed histogram of the effective potential distribution shown in Fig. 4.39 (top left). Because the amplification of the signal is proportional to voltage, the histogram represents the perfect-resolution energy response of the detector to a homogeneously distributed mono-energetic source of events.

We define the reduced NTL events to include any event whose recoil location results in a reconstructed recoil energy that differs from the true recoil energy by more than the $1 \sigma$ detector energy resolution. This corresponds to events that see less than $93.3 \%$ of the full bias voltage $V_{\text {det }}$. For electron recoils, the measured event energy is reduced from the nominal expectation according to

$$
E^{\text {measured }}=E^{\text {nominal }} \times \frac{1+\frac{\Delta V}{\epsilon_{\gamma}}}{1+\frac{V_{\text {det }}}{\epsilon_{\gamma}}},
$$

where $\Delta V$ is the potential difference experienced by charge carriers produced at the recoil location, and $V_{\text {det }}$ is the nominal potential difference.

The shape of the voltage distribution is a source of systematic uncertainty for the distribution of reduced NTLs, and to account for this we perform the same analysis with an alternate voltage distribution containing more features in the voltage spectrum from the simulation. This predicts 
a slightly higher leakage rate of reduced NTLs given the same radial cut, and gives us a handle on the systematic uncertainty on the rate of reduced NTLs we expect to pass our radial cut.

\subsubsection{Radial Distribution of Reduced NTL events}

The majority of reduced NTL events are measured only slightly lower in energy than their true energy, because the distribution of $\Delta V$ inside the detector peaks strongly at the nominal voltage. Thus the energy regions just below the strong $K$ - and $L$-shell ${ }^{71} \mathrm{Ge}$-decay peaks provide good samples of reduced NTL events, with high signal to noise which we used to determine the distribution in the radial parameter $\xi$.

We model the radial distribution of reduced NTLs by defining a region in the radial parameter $\left(\xi \in\left[-2 \times 10^{-5},+4 \times 10^{-5}\right]\right)$ outside of which we observe no reduced NTLs, and selecting events in this region within a small energy range below the $L$-shell capture peak $\left(0.7-1.2 \mathrm{keV}_{\text {ee }}\right)$. Creating a cumulative distribution function in $\xi$ for these events gives us an idea of the distribution of reduced NTLs in $\xi$. A systematic uncertainty on this distribution is estimated by removing the upper bound in $\xi$ while narrowing the energy window, which creates a distribution that predicts slightly more reduced NTLs passing the same cut.

\subsubsection{Optimizing the Radial Cut}

Combining the expected energy distributions of reduced NTL events, the voltage map model, and the resolution model for $\xi$ as a function of energy, we model the reduced NTL distribution in the energy- $\xi$ plane. We randomly sample from these distributions and thus produce a prediction for the $2 \mathrm{D}$ probability distribution of the data in these variables, which is shown in Fig. 4.40. A cut can then be set on this distribution of events to allow a certain shape and magnitude of leakage past the cut.

This specific choice of event leakage was chosen after a detailed sensitivity study. While choosing a looser cut results in a better signal efficiency (improving the analysis's sensitivity), a looser cut also results in a higher reduced NTL background leakage past the cuts (worsening the analysis's sensitivity). Monte Carlo simulations indicated that minimal extra sensitivity was gained by choosing a looser cut [116]. Therefore, in order to eliminate the need to include a reduced NTL model into the profile likelihood background model, we chose a particularly restrictive cut.

We set a cut on $\xi$ as a function of energy that results in $0.13 \pm 0.1_{\text {stat }} \pm$ 


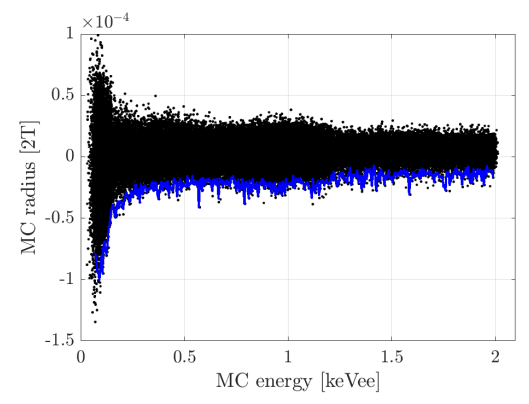

Figure 4.40: The Monte Carlo distribution of reduced NTL events in the $2 \mathrm{~T}$ radius (i.e. $\xi$ ) vs. energy plane. The colored points are those that would pass a cut such that (after normalizing the Monte Carlo to the Run 3 exposure) only $1 / 8$ th of an event would pass into the signal region. (credit: R. Underwood)

$0.44_{\text {sys }}$ reduced NTLs passing the cut. The systematic error is estimated from Monte Carlo simulations with the alternate radial and voltage models (with the radial distribution of reduced NTLs being the larger contributor). The cut boundary was chosen such that the expected distribution of reduced NTLs passing the cut is uniform in energy between 0.07 and $2 \mathrm{keV}_{\text {ee }}$. The radial parameter cut imposes an analysis threshold of $70 \mathrm{eV}_{\mathrm{ee}}$, which is determined by the lowest well-determined bound of the radial resolution model.

\subsection{Livetime and Signal Efficiency}

While the cuts described in Sec. 4.5 are designed to remove background events, they remove some good events as well. When looking for a DM signal in the data, we must account for the fact that the cuts make the analysis less than 100\% efficient to the signal. Because signal-background discrimination becomes increasingly difficult at lower energies, we calculate the efficiency as a function of energy, as described in Sec. 4.9.3.

In addition to the cuts removing good events that could potentially be from DM particle interactions, the hardware trigger of the experiment also only reads out events with a sufficiently high signal-to-noise ratio. Therefore, recoils in the detector that are below a certain energy range are never read out as events, and we account for the reduced signal rate at low energy by calculating the trigger efficiency as a function of energy. This is described 


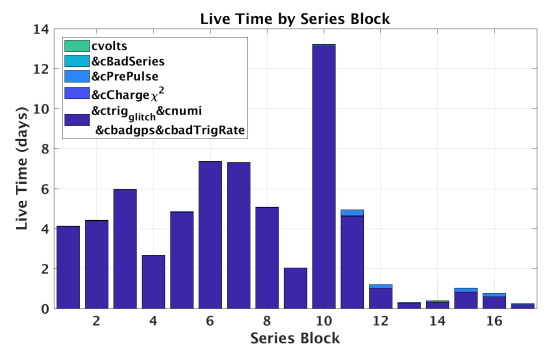

Figure 4.41: Livetime contribution of each series block to the R3 exposure, with the livetime removed by the cuts listed in Sec. 4.9.1 shown at the top of each bar of live days.

in Sec. 4.9.2.

We also consider the amount of time that the detector was "live" and able to record an event, as described in Sec. 4.9.1. The "livetime" is one component in the calculation of the magnitude with which a DM signal would appear in the detector. Ignoring annual modulation effects, the number of events observed from a DM signal increases linearly with the livetime.

\subsubsection{Livetime}

For the "livetime" of the dataset we consider the raw amount of time over which the dataset was acquired, and then account for data acquisition inefficiencies as well as removal of certain periods of time from the DM search dataset, which reduces the raw livetime.

When the data acquisition system records an event, it also records the amount of time that it was idle and waiting for that event (i.e. that event's livetime). This amount of time will be slightly less than the time between the event of interest and the previous event because the data acquisition system requires a small amount of time to read out an event that amounts to some "deadtime." Simply summing the livetime quantity for each event gives the cumulative livetime of the dataset. Without any cuts applied, the low background DM search Run 3 dataset lasted 66.9 days.

The cuts described in Sec. 4.5 remove periods of time when the detector was behaving sub-optimally. These cuts are the high voltage, NuMI beam, prepulse baseline, charge chi squared, bad series, bad GPS time, bad trigger rate, and the "T2Z1 triggered" cuts. With all R3 LT cuts applied, the total Run 3 livetime is 60.9 days. 
We compute the livetime removed for these cuts for each of the 17 series blocks, and as shown in Fig. 4.41 the cuts retain most of the livetime (though it should be noted that some of the bad series are not assigned to a series block, and so visually this plot underestimates the livetime removed by the cuts). After livetime cuts have been applied, the Run 3 total livetime is 60.9 days (or $36.4 \pm 0.3 \mathrm{~kg}$-days of exposure for the T2Z1 mass of $0.597 \pm 0.005$ $\mathrm{kg}[117])$.

\subsubsection{Trigger Efficiency}

The central idea of the trigger efficiency is that the detector's DAQ hardware only issues a trigger and reads out events whose energy deposition is large enough to create a significant increase of the signal above the baseline noise. Therefore, while very low energy events buried in the baseline noise will never cause a trigger and high energy events will always cause a trigger, there is a middle ground of events that are only slightly distinguishable above baseline noise and that the hardware trigger has a non-zero and non-unity efficiency of identifying. Trigger efficiency studies calculate this efficiency and parametrize it as a function of event energy.

The trigger efficiency is typically calculated using ${ }^{252} \mathrm{Cf}$ calibration data, as opposed to low background data, because the method for calculating the trigger efficiency improves with increased statistics. For Run 3, the trigger efficiency was calculated using the three-day ${ }^{252} \mathrm{Cf}$ calibration taken at the beginning of Run 3. The vast majority of the events that occur on the detector of interest (in this case T2Z1) were read out because they caused a trigger on T2Z1. Therefore measuring the trigger efficiency on T2Z1 with events that are known a priori to have caused a trigger is biasedthe measurement will indicate $100 \%$ trigger efficiency. Instead, the method employed to measure the T2Z1 efficiency operates on the idea that events that have caused a trigger on the other active detectors (in this case T2Z2 and T2Z3) are an unbiased sample of events that can be used to measure the T2Z1 trigger efficiency. This is because for good events, the trigger of the other detectors should not depend on the trigger of T2Z1. Of those events that caused a trigger on the other detectors, we calculate, in energy bins of $0.2 \mathrm{keV}_{\mathrm{t}}$, the fraction of events that caused a trigger on T2Z1. That fraction (labeled "efficiency" on the y axis) is plotted as a function of energy in Fig. 4.42.

To select the events for the trigger efficiency calculation, we apply restrictive cuts [118] to ensure that the measurement is being made with good events that were not due to instrumental backgrounds (e.g. low frequency 

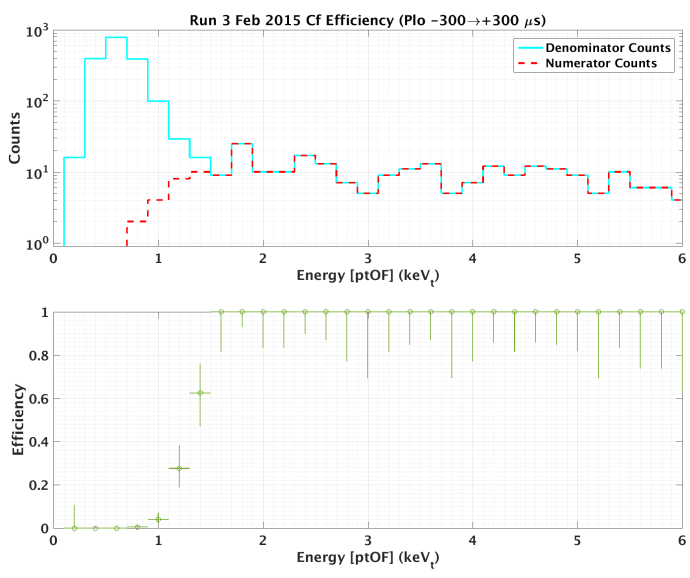

Figure 4.42: (top) Trigger calculation showing the number of events that triggered both T2Z1 and the other detectors (numerator counts) as well as the number of events that triggered just the other detectors (denominator counts). (bottom) Binned efficiency of the trigger as a function of energy. Error bars are binomial statistical uncertainties.

noise or glitch triggers). The high rate ${ }^{252} \mathrm{Cf}$ calibration data with which the trigger efficiency is measured also helps to ensure that instrumental noise events make up a small fraction of the events being used to measure the efficiency.

The trigger efficiency was parametrized as a modified error function given by $\operatorname{Efficiency}(E)=0.5\left(1+\operatorname{erf}\left(\frac{E-\mu}{\sqrt{2} \sigma}\right)\right)$ where $\mu$ corresponds to the $50 \%$ efficiency point and $\sigma$ determines the width of the curve.

Figure 4.42 shows that there is an energy range of interest between 0.7 and $1.5 \mathrm{keV}_{\mathrm{t}}$ in which some events have issued a trigger on $\mathrm{T} 2 \mathrm{Z1}$ (i.e. passing events) and some events have not issued a trigger on T2Z1 (i.e. failing events). Given the error function trigger efficiency model, at an energy $E$, the probability of an event passing is $0.5\left(1+\operatorname{erf}\left(\frac{E-\mu}{\sqrt{2} \sigma}\right)\right)$, and the probability of an event failing is $0.5\left(1-\operatorname{erf}\left(\frac{E-\mu}{\sqrt{2} \sigma}\right)\right)$. The data consists of energies of the passing events $\left(E_{\text {pass }}\right)$ and failing events $\left(E_{\text {fail }}\right)$, and so up to a normalization 

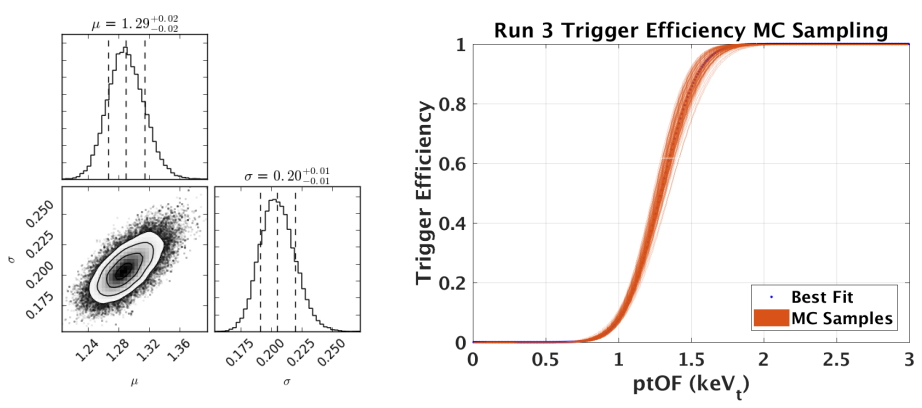

Figure 4.43: (left) Corner plot for trigger efficiency fit of $\mu$ and $\sigma$. (right) Best-fit error function with $100 \mathrm{MC}$ sample curves from the $\mu$ vs. $\sigma$ posterior. These samples serve as visualizations of the model uncertainties.

factor, the log likelihood of the data is:

$$
\begin{aligned}
\ln \mathcal{L} & =\sum_{i=1}^{N} \ln \left[0.5\left(1+\operatorname{erf}\left(\frac{E_{\text {pass }, i}-\mu}{\sqrt{2} \sigma}\right)\right)\right] \\
& +\sum_{j=1}^{M} \ln \left[0.5\left(1-\operatorname{erf}\left(\frac{E_{\text {fail }, j}-\mu}{\sqrt{2} \sigma}\right)\right)\right],
\end{aligned}
$$

where $N$ is the number passing events, and $M$ is the number of failing events.

Maximizing the likelihood gives best-fit parameters: $\mu=1.29 \mathrm{keV}_{\mathrm{t}}, \sigma=0.20 \mathrm{keV}_{\mathrm{t}}$. To evaluate posteriors, uniform priors were used on $\mu$ and $\sigma$, and the emcee [119] MCMC package was used to sample the likelihood. The covariance between model parameters, as well as the best fit model, is shown in Fig. 4.43.

\subsubsection{Signal Simulation}

The efficiencies of the glitch, phonon $\chi^{2}$, square, and BDT-based LF noise cut were computed using a simulation of good events, which we refer to as a "pulse simulation." A cartoon of the simulation procedure is shown in Fig. 4.44, where we use a noiseless pulse template for the shape of the simulated pulse, and we use a random event to represent the noise for the simulated pulse.

The CDMS BatFaker software package, written by B. Loer as part of the salting effort, as well as MATLAB tools, are used to create the fake events in a format that can be processed through the CDMS reconstruction software. BatFaker and the MATLAB tools work by replacing events in an existing 


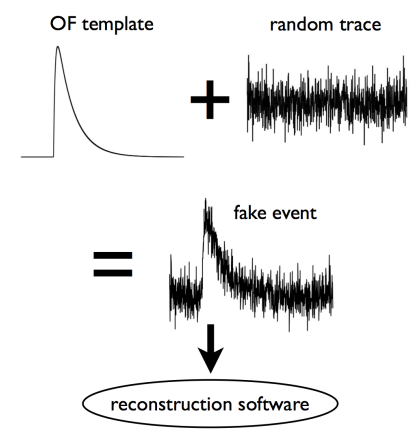

Figure 4.44: A cartoon depicting the pulse simulation procedure (credit A. Anderson: [120]).

CDMS raw data file with fake data that can be specified by the user. In particular, one creates a fake pulse by combining (1) a noise trace from a random event in the real data, (2) a slow template scaled to a user-defined energy, and (3) a fast template scaled to a user-defined amplitude.

For calculating cut efficiency as a function of energy, fake pulses are simulated that are uniformly distributed in energy. To first order, this is accomplished by uniformly varying the amplitude of the noiseless template shown in Fig. 4.44 while not scaling the noise traces. To second order, the pulse shape varies as a function of energy, which we include in the simulation. The majority of energy-dependent pulse shape variations, as well as the peaky and non-peaky position-dependent features that exist in individual channels, are averaged out when the 4 channels are summed to make the total phonon pulse; however, there are still pulse shape deviations from the standard optimal filter template. We seek to simulate these variations in our fake data using a 2-template (1 slow, 1 fast) approach.

To capture the variations in peakiness of the pulses as a function of energy, we use the ratio of fast to slow amplitude fits of good events, which is shown in Fig. 4.45 left. We also examine the raw $L$-shell traces at the extrema of the fast/slow amplitude ratio distribution in Fig. 4.45 right. These raw traces confirm that the $L$-shell events at the extreme of the ratio distribution are still good events, and the full range of the fast/slow ratio can be sampled to create fake pulses that resemble true signal. The slow amplitudes were uniformly sampled such that the pulse energies would be uniformly distributed between 0 and $60 \mathrm{keV}_{\mathrm{t}}$. To determine the relative amplitude of the fast and slow template to use for this simulated data, we use the distribution 

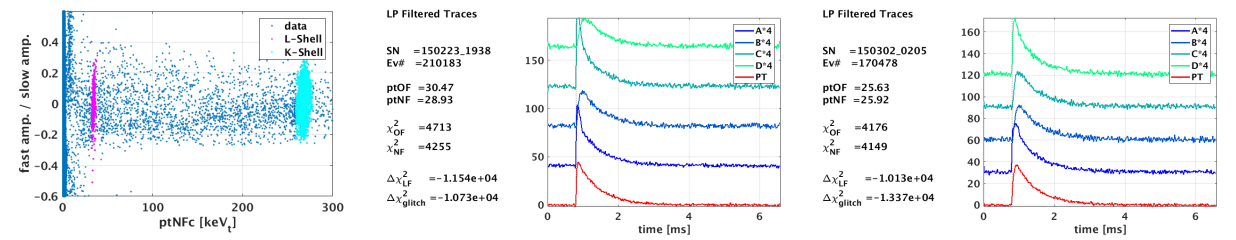

Figure 4.45: (left)The fast/slow amplitude ratio, as a function of energy, for the two-template fit optimal filter fit. (center) (right) Two $L$-shell pulses showing the variation in pulse peakiness where the center pulse has is the peakiest of the $L$-shell events (fast/slow ratio $=0.4$ ) and the right pulse is the least peaky of the $L$-shell events (fast/slow ratio $=-0.6$ ).

of the fast/slow template ratio observed in the low background data from $0-30 \mathrm{keV}_{\mathrm{t}}$, in energy bins of $2 \mathrm{keV}_{\mathrm{t}}$. The fast amplitudes were sampled from the fast/slow amplitude ratio distributions.

\subsubsection{Quality Cut Efficiency}

After creating the fake event datasets we process them through the event reconstruction software, and then examine the location of our simulated good events in the parameter spaces in which we have placed cuts. An example of the simulated events in the $\Delta \chi_{\text {glitch }}^{2}$ vs. energy plane is shown in Fig. 4.46 left, where the slope of the parabolic distribution matches the trends observed in the real data. One complication is that the glitch, phonon $\chi^{2}$, and square cuts were tuned on 17 different series blocks of data as described in Sec. 4.6, while the BDT-based cut was tuned separately for R3a and R3b as described in Sec. 4.7. Accordingly, the efficiencies of these cuts were calculated for the different series block by simulating the fake pulses with randoms selected from that series block.

The efficiency of any given cut is simply the passage fraction for a specific energy bin, the ratio of passing events to all events, where "all events" constitutes all the events that pass the livetime cuts.

Because many of the DQ cuts are based on the pulse shape and optimal filter fitting results, some correlation is expected between the cuts. For example, one would hope that clear glitch events would also fail the phonon $\chi^{2}$ cut and potentially the square pulse cut. The correlation between the different cuts is accounted for by simply computing the logical AND of all the cuts. The final efficiency for a group of data quality (DQ) cuts, for each 

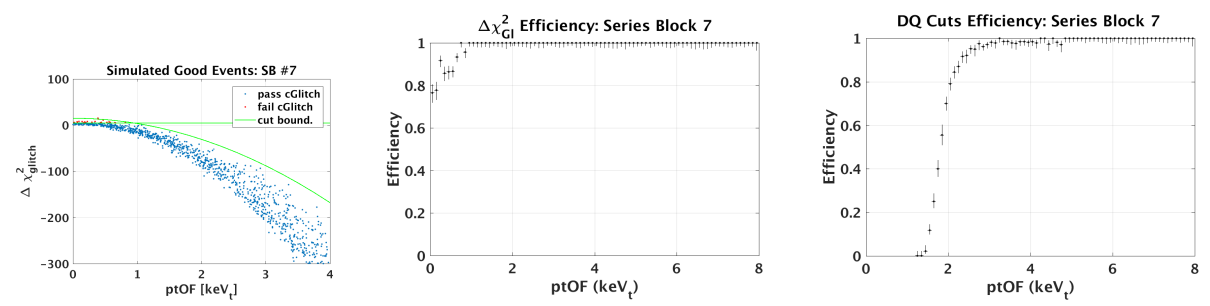

Figure 4.46: (left) The distribution of simulated signal events in the $\Delta \chi_{\text {glitch }}^{2}$ vs energy plane with the cut boundary overlaid. (center) The efficiency of the $\Delta \chi_{\text {glitch }}^{2}$ cut calculated with data the simulated signal data. (right) The efficiency of all DQ cuts calculated using Eq. 4.26. All figures use series block 7 simulated data and cut boundaries.

energy bin, is then

$$
\text { Efficiency }=\operatorname{sum}\left[\operatorname{cuts}_{\mathrm{LT}} \& \operatorname{cuts}_{\mathrm{DQ}}\right] / \operatorname{sum}\left[\operatorname{cuts}_{\mathrm{LT}}\right],
$$

where the data quality cuts are collectively labeled as cuts $\mathrm{DQ}_{\mathrm{DQ}}$ and the live-

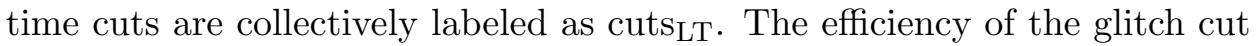
alone and for all DQ cuts, for series block 7, are shown in Fig. 4.46 center and left respectively.

The error bar for each energy bin is the $1 \sigma$ width of a binomial distribution with the central value (probability of passing) $p$, the number of total events for each bin is $N$, and the number of events passing the cut in that energy bin is given by $k$. This distribution is given by:

$$
B(k ; N, p)=\left(\begin{array}{c}
N \\
k
\end{array}\right) p^{k}(1-p)^{N-k}=\frac{N !}{k !(N-k) !} p^{k}(1-p)^{N-k} .
$$

Using the binomial distribution to estimate the uncertainty breaks down when $p=1$ and $p=0$, where the variance of the binomial distribution vanishes, unrealistically indicating $100 \%$ certainty of the efficiency measurement. Alternatives for computing sensible uncertainty bands for these cases have been proposed that employ Bayes' theorem. Using a uniform prior on $p$ and a binomial distribution as the likelihood, one can analytically solve for the posterior on $p$ :

$$
P(p ; k, N)=(N+1) B(k ; N, p) .
$$

as derived in Ref. [121]. Equation 4.28 does not result in zero variance when $k=0$ and $k=N$, and so we use this result for the two edge cases. 


\subsubsection{Fiducial Volume Efficiency}

The final fiducial volume efficiency is the fraction of events that are reconstructed at the correct energy that pass the radial cut. In a method developed by W.Rau, R. Underwood, and K. Page [122], the radial cut efficiency is split up into two parts. The first part, called the "energy efficiency," is the fraction of events reconstructed at the correct energy. The second part, called the "peak efficiency," is the fraction of events at a given energy that pass the radial cut. The energy efficiency and peak efficiency are given by the first term and second term, respectively, on the right-hand-side of Eq. 4.29:

$$
\text { Efficiency }=\frac{P_{i}}{R+P_{o}+P_{i}}=\frac{P_{o}+P_{i}}{R+P_{o}+P_{i}} \times \frac{P_{i}}{P_{o}+P_{i}} .
$$

The notation of Equation 4.29 is defined in Fig. 4.39 top right.

The "energy efficiency" was measured by finding the ratios of $K-, M$ , and $L$ - shells events with misreconstructed energy to those events with correctly reconstructed energy. The number of misreconstructed $K-, M-$, and $L$ - shell events was estimated using a time-dependent fit that exploited the fact that the rate of the $K-, M$-, and $L$ - shell backgrounds decay with a 11.43 day half-life, the half-life of ${ }^{71} \mathrm{Ge}$, while other dominant backgrounds decay over significantly longer timescales or not at all. Details of the energy efficiency calculation can be found in Ref. [82].

The "peak efficiency" can be measured with techniques similar to those used to construct the reduced NTL model in Sec. 4.8. We use a Monte Carlo simulation based on the radial resolution model to simulate the radial parameter distribution for events having the full NTL amplification. Because the DM signal will be uniformly distributed in the detector, we model the $\xi$ distribution for uniformly distributed, full NTL amplification events with reconstructed energies in the $L$-shell line. We statistically subtract the small contribution of non- ${ }^{71} \mathrm{Ge}$ backgrounds from this distribution and deconvolve the radial-parameter resolution at the $L$-shell line energy $\left(1.3 \mathrm{keV}_{\mathrm{ee}}\right)$. The deconvolved distribution is expected to be the underlying "true" distribution of $\xi$ for events at the $L$-shell energy. We then use the resolution model of $\xi$, described in Sec. 4.8, to scale this distribution according to energies from 0.07 to $2 \mathrm{keV}_{\text {ee }}$, thereby creating energy-dependent probability distributions for $\xi$. We then apply the radial cut to these simulated distributions, and by doing so obtain the efficiency of the fiducial volume cut for events with full NTL amplification. 


\subsubsection{Combining and Parametrizing the Efficiency}

The trigger efficiency, quality cut efficiency, and the radial cut efficiency are combined by multiplying the mean value of the efficiencies together and using Monte Carlo to propagate the uncertainty between the different sources.

For the quality cut efficiency, systematic differences between the efficiencies from the different series blocks require propagating the uncertainty between series blocks to obtain a combined total efficiency curve for all quality cuts and all series blocks. To generate a Monte Carlo (MC) sample efficiency curve, for each energy bin a random number is drawn from a binomial distribution using the central value (probability of passing) $p$ and number of total events $N$ for each bin. Visually, this means sampling the distribution described by the error bars in Fig. 4.46 right independently for each energy bin. In order to properly weight the efficiency contribution from each series block, the number of MC efficiency curves created for each series blocks is linearly proportional to that series' livetime. Many MC curves are generated and multiplied together to determine the mean efficiency values as well as the $1 \sigma$ efficiency uncertainty.

For the trigger efficiency, we sample the $2 \mathrm{D}$ posterior for the trigger efficiency model $\mu$ and $\sigma$. The posterior is shown in Fig. 4.43 left while the MC sampled trigger efficiency curves are shown in Fig. 4.43 right. The trigger efficiency curves are incorporated into the total efficiency by multiplying them by the quality cut MC efficiency curves, although it is clear that the trigger efficiency is a subdominant contribution. That is, the quality cut efficiency falls to a low value at $1.75 \mathrm{keV}_{\mathrm{t}}$ where the trigger has high efficiency.

The total efficiency curve is parametrized with an error function given by

$$
\operatorname{Efficiency}(E)=A\left(1+\operatorname{erf}\left(\frac{E-\mu}{\sqrt{2} \sigma}\right)\right)
$$

and the results of the fit of that model are shown in Fig. 4.47. 


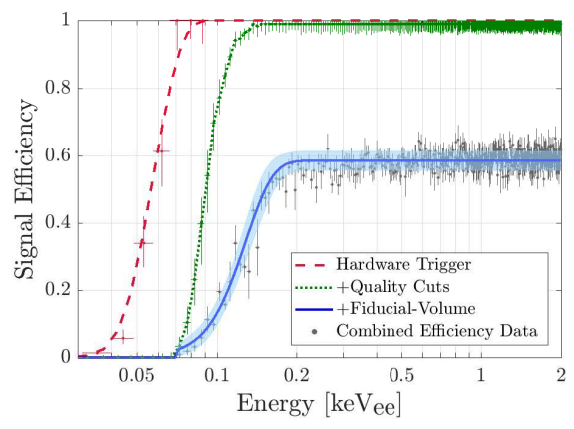

Figure 4.47: The signal efficiency with successive application of the trigger efficiency, quality cuts efficiency, and fiducial volume cut efficiency. The final data is included with statistical and systematic $1 \sigma$ uncertainty. Fitting the efficiency model to these data gives the final (blue) efficiency curve and the corresponding $\pm 1 \sigma$ uncertainty band. 


\section{Chapter 5}

\section{Profile Likelihood Dark Matter Search with CDMSlite Run 3}

The final chapter of this thesis focuses on the aspects of the Run 3 analysis that deviated sharply from past CDMSlite analyses and allowed us to employ more powerful statistical methods to look for a DM signal in the Run 3 dataset. We start the chapter by discussing background models for all of the known backgrounds in the CDMSlite Run 3 region of interest (ROI). We also develop a signal model, and then characterize the dominant systematic uncertainties of the analysis. These systematic uncertainties in turn allow the signal and background models to change shape (within a systematic uncertainty range) in the fit. Then, after unblinding (or "unsalting") the data and observing no DM-like component in the data, we employ the profile likelihood framework that uses a likelihood ratio statistic to set a limit on a DM signal in a way that naturally incorporates systematic uncertainties into the limit. Our approach of accounting for known backgrounds has significant benefits over the optimum interval approach (discussed in Sec. 2.5.3) of the previous CDMSlite analysis. Namely, the background models and profile likelihood method have the benefits of (1) improving the limit over the OI, and (2) moving CDMSlite from an exclusion-only analysis into one with discovery potential.

\subsection{Background Models}

The SuperCDMS cryostat was surrounded by layers of shielding that blocked almost all external radiation, such as $\gamma$-rays and neutrons from the cavern walls, and as a result the dominant source of background particles in the detector came from radioactivity of the shielding, other apparatus materials, and the detectors themselves. The profile likelihood analysis, discussed in more detail in Sec. 5.2, requires probability distribution functions (PDFs) 
for every background component that will contribute events to the DMsearch data. In addition to the PDF that defines the spectral shape of the background, if the number of events from a background component is known, the expected number of events contributing from that background (i.e. the normalization) can be included in the profile likelihood fit.

The primary backgrounds modeled for this analysis are cosmogenic activation of the crystal, neutron activation from ${ }^{252} \mathrm{Cf}$ calibration, Compton scattering from primordial isotopes in the apparatus materials, and ${ }^{210} \mathrm{~Pb}$ contamination on the surfaces of the detector and its copper housing. We use Monte Carlo simulations, as well as data-driven fits, to model these backgrounds. Table 5.1 lists the considered background components and whether or not the information about the normalization was included in the likelihood fit. These background models were developed by D. Barker, and below we briefly describe each background for completeness. We direct the reader to D. Barker's thesis [123] for a more thorough discussion of the background model development. In particular, D. Barker discusses the applicability of the GEANT4 simulation to package to the low energies (50 eV-2 keV) of interest to this analysis through validation of the low-energy electromagnetic physics lists in GEANT4.

\subsubsection{Energy Resolution Model}

We require a good model of the energy resolution in order to calculate the expected energy spectra for background models. We model the total CDMSlite energy resolution as in Ref. [82]:

$$
\begin{aligned}
\sigma_{\mathrm{T}}\left(E_{\mathrm{r}, \mathrm{ee}}\right) & =\sqrt{\sigma_{\mathrm{E}}^{2}+\sigma_{\mathrm{F}}^{2}\left(E_{\mathrm{r}, \mathrm{ee}}\right)+\sigma_{\mathrm{PD}}^{2}\left(E_{\mathrm{r}, \mathrm{ee}}\right)} \\
& =\sqrt{\sigma_{\mathrm{E}}^{2}+B E_{\mathrm{r}, \mathrm{ee}}+\left(A E_{\mathrm{r}, \mathrm{ee}}\right)^{2}} .
\end{aligned}
$$

The energy-independent term $\sigma_{\mathrm{E}}$ describes the baseline resolution and accounts for electronics noise and any drift in the operating conditions. The Fano term $\sigma_{\mathrm{F}}$ accounts for fluctuations in the number of generated charges [125] and is proportional to $\sqrt{E_{\mathrm{r}, \mathrm{ee}}}$. The $\sigma_{\mathrm{PD}}$ term reflects the position dependence of the event within the detector due to the electric field, TES response, etc., and is proportional to $E_{\mathrm{r}, \text { ee }}$. Separating out the energy dependence we end up with the three model parameters $\sigma_{\mathrm{E}}, B$, and $A$.

We use several measurements to determine the resolution model for Run 3. We use randomly triggered events to determine the zero-energy noise distribution. Additionally we use the widths of the $K$-, $L$-, and $M$-shell ${ }^{71} \mathrm{Ge}$ 


\begin{tabular}{l|r}
\hline Component & $\begin{array}{r}\text { Normalization Constrained } \\
\text { vs. Free, and Other Comments }\end{array}$ \\
\hline Compton & Free \\
Tritium $\left({ }^{3} \mathrm{H}\right)$ & Free \\
${ }^{71} \mathrm{Ge}$ & Free \\
${ }^{68} \mathrm{Ga}$ & Free \\
${ }^{65} \mathrm{Zn}$ & Free \\
${ }^{65} \mathrm{Fe}$ & Free \\
Surface Germanium & Constrained \\
Surface Housing & Constrained \\
Surface Top Lid & Constrained \\
neutrons & Not included \\
& (contributes $<1$ event [124]) \\
low rate isotopes $\left({ }^{60} \mathrm{Co},{ }^{54} \mathrm{Mn},{ }^{49} \mathrm{Vn}\right)$ & Not included \\
& (contributes $<1$ event) \\
Instrumental Backgrounds & Not included \\
\end{tabular}

Table 5.1: Background components considered in the likelihood fit 
5.1. Background Models

\begin{tabular}{|c|c|c|c|}
\hline$\overline{\text { Peak }}$ & & $\begin{array}{c}\text { Energy } \\
\mu\left[\mathrm{keV}_{\mathrm{ee}}\right]\end{array}$ & $\begin{array}{c}\text { Resolution } \\
\sigma\left[\mathrm{eV}_{\text {ee }}\right]\end{array}$ \\
\hline$K$ shell & & $10.35 \pm 0.002$ & $108 \pm 2$ \\
\hline$L$ shell & & $1.33 \pm 0.003$ & $36.3 \pm 2.0$ \\
\hline$M$ shell & & $0.162 \pm 0.002$ & $13.9 \pm 2.0$ \\
\hline \multirow{2}{*}{ Baseline } & Period 1 & 0.0 & $9.87 \pm 0.04$ \\
\hline & Period 2 & 0.0 & $12.7 \pm 0.04$ \\
\hline
\end{tabular}

Table 5.2: Reconstructed energies and resolutions of the ${ }^{71} \mathrm{Ge}$ decay peaks and the baseline noise in CDMSlite Run 3.

\begin{tabular}{lccl}
\hline & $\sigma_{\mathrm{E}}\left[\mathrm{eV}_{\mathrm{ee}}\right]$ & $B\left[\mathrm{eV}_{\mathrm{ee}}\right]$ & $A\left(\times 10^{3}\right)$ \\
\hline Period 1 & $9.87 \pm 0.04$ & $0.87 \pm 0.12$ & $4.94 \pm 1.27$ \\
Period 2 & $12.7 \pm 0.04$ & $0.80 \pm 0.12$ & $5.49 \pm 1.13$ \\
\hline
\end{tabular}

Table 5.3: Best-fit energy resolution parameters of the model in Eq. 5.2 for Period 1 and Period 2.

activation peaks to determine the energy dependence of the resolution. We fit these peaks with a combination of a Gaussian and linear background model in order to determine the width of the peaks.

Table 5.2 gives the peak position $\mu$ and resolution $\sigma$ of each ${ }^{71} \mathrm{Ge}$ peak, as given by the Gaussian fits to the peaks shown in Fig. 5.1. Because the zero-energy baseline resolution varies with the applied bias voltage and with environmental conditions, all of which changed between Period 1 and Period 2, we calculate separate livetime-weighted average resolutions for each period. These are given in Table 5.2. The measured widths of the $K$-, $L$-, and $M$-shell peaks are consistent between Period 1 and Period 2, and so common values are used for both periods.

We apply this energy-dependent resolution model when calculating the expected energy distribution for the background and DM signal components. We propagate uncertainties in the model parameters as systematic uncertainties in the profile likelihood fit of Sec. 5.2.

\subsubsection{Cosmogenic Activation}

Cosmic rays that strike the detectors (during fabrication, storage, and transportation above ground) activate the crystals. In germanium detectors, cosmogenically produced tritium is a significant background, with contributions from other isotopes that decay primarily either by $\beta$-decay or electron capture (EC). The isotopes that undergo EC give discrete peaks in the detectors 


\subsection{Background Models}

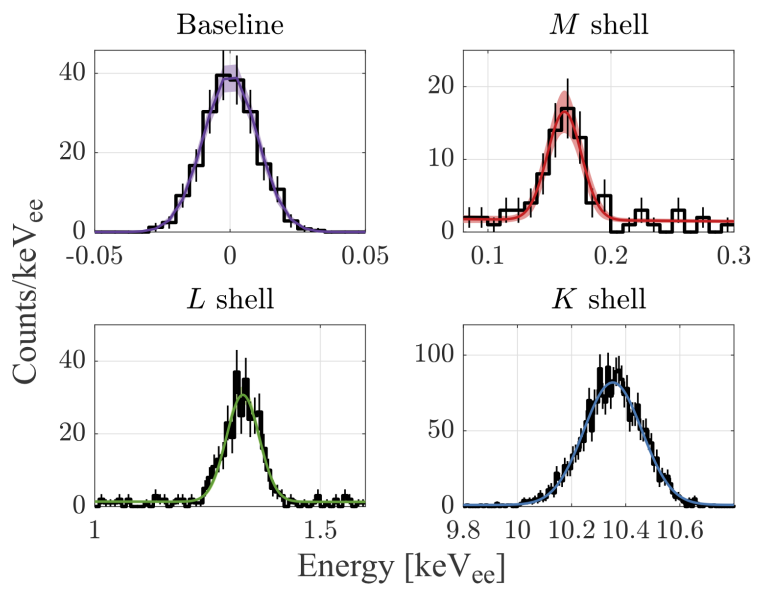

Figure 5.1: Fits of a Gaussian + linear background to the energy spectra of zero-energy (baseline) events and events from each ${ }^{71} \mathrm{Ge}$ activation peak. The widths of the Gaussians are the energy resolution $\sigma$. Copied from [126] with permission. (credit: D. Barker)

below $\sim 10 \mathrm{keV}$ and were observed in the CDMSlite Run 2 spectrum [127]. We describe analytic models for the tritium beta-decay spectrum and the EC lines.

\section{Tritium}

Non-relativistic $\beta$-decay theory describes the tritium's decay spectrum because its endpoint, or $Q$-value, satisfies the relationship $Q \ll m_{e} c^{2}$, where $m_{e}$ is the electron mass. The distribution of the electron's kinetic energy $E_{\mathrm{KE}}$ is described by

$$
\begin{aligned}
f_{\text {tritium }}\left(E_{\mathrm{KE}}\right)= & C \sqrt{E_{\mathrm{KE}}^{2}+2 E_{\mathrm{KE}} m_{e} c^{2}}\left(Q-E_{\mathrm{KE}}\right)^{2} \\
& \times\left(E_{\mathrm{KE}}+m_{e} c^{2}\right) F\left(Z, E_{\mathrm{KE}}\right),
\end{aligned}
$$

where $C$ is a normalization constant and $F\left(Z, E_{\mathrm{KE}}\right)$ is the Fermi function [128]. The non-relativistic approximation for the Fermi function is given by

$$
F\left(Z, E_{\mathrm{KE}}\right)=\frac{2 \pi \eta}{1-e^{-2 \pi \eta}}, \text { with } \eta=\frac{\alpha Z\left(E_{\mathrm{KE}}+m_{e} c^{2}\right)}{p c} .
$$


5.1. Background Models

\begin{tabular}{lcccccc}
\hline Shell: & \multicolumn{2}{c}{$K$} & \multicolumn{2}{c}{$L_{1}$} & \multicolumn{2}{c}{$M_{1}$} \\
& $\mu$ & $\Lambda$ & $\mu$ & $\Lambda$ & $\mu$ & $\Lambda$ \\
\hline${ }^{68} \mathrm{Ga}$ & 9.66 & 1.0 & 1.20 & 0.1107 & 0.140 & 0.0183 \\
${ }^{65} \mathrm{Zn}$ & 8.98 & 1.0 & 1.10 & 0.1168 & 0.122 & 0.0192 \\
${ }^{55} \mathrm{Fe}$ & 6.54 & 1.0 & 0.77 & 0.1111 & 0.082 & 0.0178 \\
\hline
\end{tabular}

Table 5.4: Cosmogenic isotopes that decay via electron capture and are present in the measured CDMSlite spectrum. The shell energies $\mu$, given in $\mathrm{keV}$, are from Ref. [130]. The amplitudes $\Lambda$, from Ref. [131], are normalized with respect to the $K$ shell.

Here $Z$ is the atomic number of the daughter nucleus, $\alpha$ is the fine structure constant, and $p$ is the electron's momentum [129]. The analytical description given by Eqs. 5.3 and 5.4 describes the tritium background used for the likelihood analysis.

\section{Electron Capture Peaks}

The cosmogenic isotopes that decay via EC and are present in the measured CDMSlite spectrum are listed in Table 5.4 with their shell energies and relative amplitudes, normalized to the $K$ shell. The observed energy distribution is a Gaussian peak at the energy of the respective shell with a width given by the detector's energy resolution.

In our background model, the amplitude ratio between the $K-, L$ - and $M$-shell peaks is assumed to be as given in Table 5.4. The contribution of each EC isotope to the spectrum is given by an equation of the type

$$
f_{\mathrm{ECpeaks}}(E)=\sum_{i=K, L, M} \frac{\Lambda_{i}}{\sigma_{i} \sqrt{2 \pi}} \exp \left[-\frac{1}{2}\left(\frac{E-\mu_{i}}{\sigma_{i}}\right)^{2}\right],
$$

where $\Lambda_{i}$ are the amplitudes of the respective shells, $\mu_{i}$ are the shell energies, and $\sigma_{i}$ are the energy resolutions at the respective energies.

By modeling the EC peaks with Eq. 5.5, the number of events in the $K$ shell is the only free parameter in the likelihood fit, with the other peak amplitudes determined from the branching ratios.

\subsubsection{Electron Capture of ${ }^{71} \mathrm{Ge}$}

The ${ }^{71}$ Ge EC peaks, which are used to calibrate the energy scale and have been previously discussed in Sec. 2.5, are also a source of background. Therefore this component is included in the background model. They are modeled 
using the same functional form as the cosmogenic EC peaks (Eq. 5.5) with the one exception that, due to the large overall number of events, the $\mathrm{L}_{2}$ peak is not negligible and is thus included in the fit. This component, omitted from Table 2.5, has an energy of $1.14 \mathrm{keV}$ and relative amplitude of 0.0011 .

\subsubsection{Compton Scattering}

The dominant contributors to the Compton background are the radiogenic photons from trace amounts of contamination in the experimental materials. These originate from the shield materials (polyethylene and lead) as well as the cryostat and towers (copper). Typically the energy distribution of the recoiling electrons is assumed to be uniform, or "flat," however for the low energies of CDMSlite, atomic binding energy effects create a measurable deviation from a flat Compton energy spectrum. In particular, in both germanium and silicon "Compton steps," which are step-like features created in the energy spectrum because the detector collects at least the binding energy of any freed electron, have been observed. The Monash Compton Model [132] accounts for the atomic binding energy in the calculation of the energy of the scattered incident photon and the detector's recoiling electron.

For example, the electrons in the $K$ shell of germanium have a binding energy of $11.1 \mathrm{keV}$. This energy is deposited in the detector due to the reorganization of the electron shells, along with any additional energy that is given to the freed electron by the incident gamma. Thus, an electron scattered from the $K$ shell can never deposit less than $11.1 \mathrm{keV}$ in the detector, and likewise for electrons in the other atomic shells.

While the expected location of the steps agrees with measurements by other experiments, there is more uncertainty surrounding the size of the steps. Naïvely we would expect the number of electrons in each shell to determine the relative size of the steps; however details of the electron wave functions can also affect the step size. The Compton steps have been directly observed in silicon detectors [133]. In germanium, only the $K$-shell step has been measured directly, and so other methods must be used to estimate the lower energy steps [134].

To estimate the shape of this particular background, we carried out a GEANT4 simulation [135-137] of ${ }^{238} \mathrm{U}$ decays. We fit a model consisting of a sum of error functions,

$$
f_{C}(E)=\Lambda_{0}+\sum_{\substack{i=K, L \\ M, N}} 0.5 \Lambda_{i}\left(1+\operatorname{erf}\left[\frac{E-\mu_{i}}{\sqrt{2} \sigma_{i}}\right]\right),
$$




\begin{tabular}{cccc}
\hline$\Lambda_{K}$ & $\Lambda_{L}$ & $\Lambda_{M}$ & $\Lambda_{N}$ \\
\hline $5.7 \pm 0.3$ & $15.2 \pm 0.5$ & $9.43 \pm 1.40$ & $18.7 \pm 1.3$ \\
\hline
\end{tabular}

Table 5.5: Compton model parameters for CDMSlite, normalized over the energy range $0-20 \mathrm{keV}$. All values have been multiplied by a factor of $10^{3}$ and are in units of $\mathrm{keV}^{-1}$.

to the the simulated events that scatter once in the CDMSlite detector. The location of each step is given by $\mu_{i}$, while $\sigma_{i}$ is the energy resolution at that energy given by the energy resolution model of Sec. 5.1.1. The $\Lambda_{i}$, the amplitudes of the error functions, are the relative step sizes, and are chosen so that Eq. 5.6 is normalized to one over the energy range $0-20 \mathrm{keV}$. The constant term $\Lambda_{0}$ in Eq. 5.6 has a value of $0.005 \mathrm{keV}^{-1}$ and accounts for a flat background required to fit the simulated spectrum.

Table 5.5 gives the final parameters of our Compton model, extracted from a fit of Eq. 5.6 to the GEANT4 simulation.

\subsubsection{Surface Backgrounds}

Surface events are primarily due to the decay of ${ }^{210} \mathrm{~Pb}$, which is a long-lived daughter of ${ }^{222} \mathrm{Rn}$. Radon exposure can cause ${ }^{210} \mathrm{~Pb}$ to become implanted into the surfaces of the detectors and their surrounding copper housings. Radiation from the ${ }^{210} \mathrm{~Pb}$ decay chain consists primarily of betas, Auger electrons, ${ }^{206} \mathrm{~Pb}$ ions, and alphas which have a small mean free path in Ge and will deposit the majority of their energy within a few millimeters of the detector's surface. To understand this background and build a model of its expected distribution in energy, we use a GEANT4 simulation and a detector response function. We normalize the predicted rate of surface backgrounds using a study of alphas in SuperCDMS iZIP data. Again, this effort was led by D. Barker, and we direct the reader to D. Barker's thesis [123] for a detailed description of this work.

\section{Simulation of ${ }^{210} \mathrm{~Pb}$ Contamination}

In Geant4, we use the Screened Nuclear Recoil physics list [138] to model the implantation of ${ }^{210} \mathrm{~Pb}$ into the material surfaces along with any recoil of nuclei by subsequent decays to the stable isotope ${ }^{206} \mathrm{~Pb}$. We consider three locations from where surface events may originate: the copper directly above the detector ("top lid", TL), the cylindrical housing $(\mathrm{H})$ and the surface of the germanium crystal itself $(\mathrm{Ge})$. 
We simulated energy deposition from the decays of ${ }^{210} \mathrm{~Pb},{ }^{210} \mathrm{Bi}$, and ${ }^{210} \mathrm{Po}$ for the three locations. We then apply a detector response function to each simulated decay. The detector response model uses the voltage map of Fig. 4.39 and the resolution model of Eq. 5.2 to approximate the total phonon energy measured in the detector. The response model outputs the expected surface background spectra for this analysis, used in the likelihood fit. The spectral shapes of the three surface backgrounds are shown in Fig. 5.10.

\section{Normalization}

We normalize the surface background rate with an independent measurement of the alpha decay events in the CDMSlite detector, using a data set with a livetime of $\sim 380$ days taken with the detector operated in iZIP mode. Because this iZIP-mode data set provides more detailed information on event positions, the observed rates could be attributed to surface event sources originating from parents on the top lid, housing, and detector surface. The detector surface rate is deduced from the surface facing the neighboring detector. This rate is then subtracted from the event rate measured on the side wall and the surface facing the top lid to determine the rate from the other two locations ( $\mathrm{H}$ and $\mathrm{TL}$ ). The single-scatter events that pass the voltage cut in the simulation are then scaled to the Run 3 livetime to get the expected number of surface events. The germanium, housing, and top lid are estimated to respectively contribute 3.4, 6.5, and 17 events from 0-2 $\mathrm{keV}_{\text {ee }}$ after signal efficiency cuts have been applied.

\section{Systematic Uncertainties}

There are two main sources of systematic uncertainty on the energy spectra for surface events: uncertainties in the voltage map that determines the voltage $\Delta V$ for each event, and the location of the fiducial volume cut. The map in Fig. 4.39 assumes no additional detectors in the tower. Including the detector beneath the CDMSlite detector results in a difference of $0.5 \mathrm{~V}$ and $1 \mathrm{~V}$ for the top and bottom faces respectively, which we incorporate as a systematic uncertainty. Additionally, we model uncertainties in the fiducial volume cut (using the voltage cut $V_{\text {cut }}$ as a proxy for the radial parameter cut) by varying the voltage cut from roughly $V_{\text {cut }}-2 \mathrm{~V}$ to $V_{\text {cut }}+1 \mathrm{~V}$. Figure 5.2 shows the spectra and the $1 \sigma$ uncertainty from the voltage map and voltage cut systematics. Section 5.2.1 discusses how these systematic uncertainties are incorporated into the likelihood. 


\subsection{Likelihood and Limit Setting}
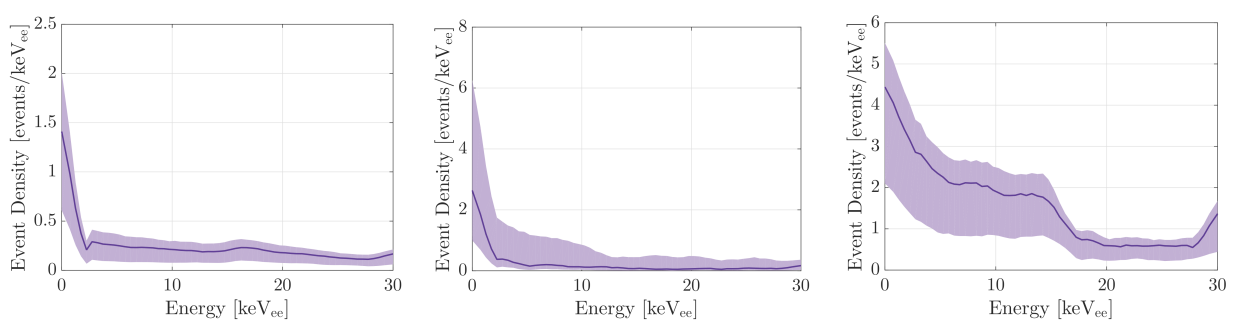

Figure 5.2: The spectra (normalized to event density) of surface events expected from the three surface background locations (left: germanium; center: housing; right: top lid). For each location, the solid curve represents the mean of the expected event distribution $\left(\rho_{0}\right)$. The shaded band shows the $1 \sigma$ uncertainty, where the top and bottom edges of the bands correspond to $\rho_{+}$and $\rho_{-}$in Eq. 5.7, respectively. Copied from [126] with permission. (credit: D. Barker)

\subsection{Likelihood and Limit Setting}

The understanding of backgrounds in the CDMSlite energy range $\left(0-25 \mathrm{keV}_{\text {ee }}\right)$ was greatly improved in the analysis of the CDMSlite R2 dataset, and with this improved understanding, background modeling likelihood approaches were used for the CDMSlite Run 3 WIMP-search analysis. This likelihood analysis of Run 3 data improves upon the Run 2 analysis for two main reasons. First, it provides improved sensitivity over the optimum interval method. Second, the optimum interval method only allows one to exclude a signal whereas in likelihood analyses the signal is included in a fit and therefore a likelihood analysis has discovery potential. For the R3 analysis we employ the profile likelihood ratio (PLR) method [139], which has the additional benefit of naturally incorporating systematic uncertainties into signal and background models and reflecting those systematic uncertainties in the sensitivity.

\subsubsection{Components Needed for the Likelihood}

The two most fundamental components of the likelihood are the data and the model. The data are the energy (in $\mathrm{keV}_{\mathrm{ee}}$ ) of the unblinded events that pass all quality cuts. The time values of the events are also used to determine if the event is from Run 3a or Run 3b (since the resolution are different for R3a and R3b as described in Sec. 5.1.1), so that events from R3a and R3b will be treated separately in the likelihood. For testing the likelihood prior 


\subsection{Likelihood and Limit Setting}

to unblinding, we also created fake datasets (i.e. pseudo-datasets) that are representative of our expectations for the final dataset. Because this analysis is focused on WIMPs between 1 and $10 \mathrm{GeV} / c^{2}$, and the energy spectrum of a WIMP signal in this mass range above $2 \mathrm{keV}_{\text {ee }}$ is small relative to the signal below $2 \mathrm{keV}_{\text {ee }}$, only data in the $0-2 \mathrm{keV}_{\text {ee }}$ range is considered for the final likelihood fit. The higher energy $5-25 \mathrm{keV}_{\text {ee }}$ region, where WIMPs of $1-10 \mathrm{GeV} / c^{2}$ contribute negligibly to the event rate, is used to generate a background rate estimation which is used in the sensitivity estimate.

The model's fit parameters allow both the magnitude and the shape of these distributions to vary, though for the surface backgrounds the normalization will be constrained within a range determined in Sec. 5.1.5. There are a total of 20 model parameters that we fit for in the likelihood: the number of background events from (1) Compton scatters; beta or EC decays from (2) ${ }^{3} \mathrm{H},(3) \mathrm{Ge}$, (4) Ga, (5) Zn, (6) Fe; (7-9) morphing parameters that determine the number of events from the surface backgrounds as well as the the surface background shapes (see discussion below for morphing parameter details); (10-12) parameters describing the signal efficiency; (1318) parameters describing the detector resolution; (19) the Lindhard $k$ value (see Sec. 2.1.2 for additional details); (20) the WIMP cross section. The first 6 parameters are allowed to float freely in the fit. Parameters 7-19 are fit including constraint terms from prior information, and the nature of these constraints is described below. Parameter 20 - the WIMP cross sectionwill sometimes be freely floating and sometimes be clamped to a specific cross section as is customary in the PLR method, and will be described below.

Of the 20 parameters listed above, 13 account for the systematic uncertainties. Explicitly these are the 3 parameters describing the signal efficiency, 6 parameters describing the resolution, 1 parameter (the Lindhard $k$ parameter) describing the yield function, and the 3 morphing parameters that take into account the systematics on the shape and normalization of the surface background.

In addition to the 20 parameters, the likelihood function also contains background distributions and a signal distribution for a given WIMP mass. These distributions are plotted in Fig. 5.3. Figure 5.3 left shows the signal distribution for 3 different values of the Lindhard $k$ (the Lindhard model is described in Sec. 2.5). Figure 5.3 right shows background distributions with no efficiency corrections applied, in units of differential rate (events $/ \mathrm{kg} /$ day, or DRU), of the 9 different background components used in the likelihood fit. The rates are the central value background rates extrapolated to $0-2 \mathrm{keV}_{\mathrm{ee}}$ from a fit to the backgrounds in the $5-25 \mathrm{keV}_{\text {ee }}$ range. 

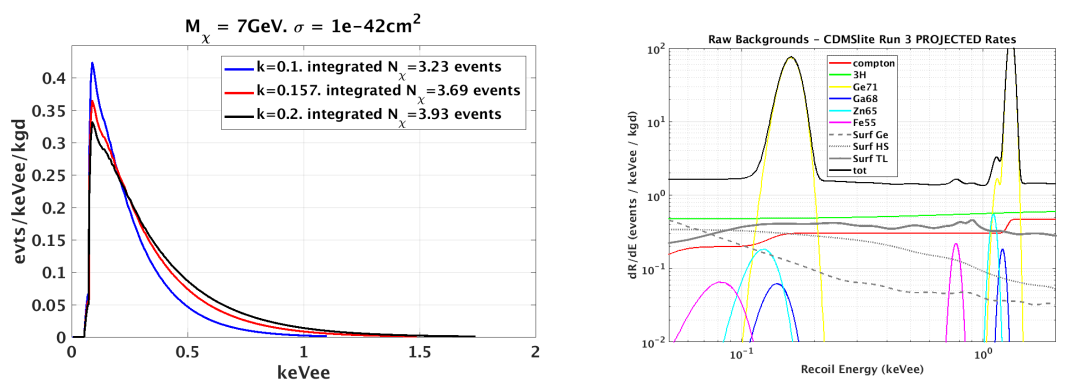

Figure 5.3: (left) The distribution expected (with detector efficiency applied) from a $7 \mathrm{GeV} / c^{2} \mathrm{DM}$ particle for different values of the Lindhard $k$ parameter. (right) Background distributions at the magnitude expected in the Run 3 data (estimated prior to unblinding).

\section{Surface Background Morphing Parameters}

The surface background components (Surface Germanium, Surface Housing, and Surface Top Lid) deserve additional discussion because they are treated differently than the non-surface backgrounds in two ways. First, the alpha-count study described in Sec. 5.1.5 provides prior information on the rate of this background; we constrain the rate of the surface backgrounds with a Gaussian prior according to the alpha rates study. Second, as shown in Fig. 5.4, there is significantly larger uncertainty on the spectral shape of the surface backgrounds than on non-surface backgrounds. As explained in Sec. 5.1.5, this is due to systematic uncertainties in the modeling of the voltage cut and the variations in the voltage map for the surface backgrounds; the voltage cut and map modeling systematics do not arise for the other backgrounds which are more homogeneously distributed throughout the detector. Whereas the shapes of the non-surface backgrounds have only small freedom to change in the likelihood fit (due to efficiency and resolution parameters), we allow the surface background shapes to shift significantly if the data pulls the surface distributions away from the mean distributions.

This shape-shifting and rate-shifting is implemented with a "morphing parameter," which acts as a fit parameter in the likelihood. With three curves giving the mean, $1 \sigma$ upper, and $1 \sigma$ lower event densities of a background component, the morphing parameter $m$ is implemented as:

$$
\rho(E, m)= \begin{cases}\rho_{\text {med }}(E)+m \times\left(\rho_{\text {up }}(E)-\rho_{\text {med }}(E)\right) & m>0 \\ \rho_{\text {med }}(E)+m \times\left(\rho_{\text {med }}(E)-\rho_{\text {low }}(E)\right) & m<0\end{cases}
$$




\subsection{Likelihood and Limit Setting}
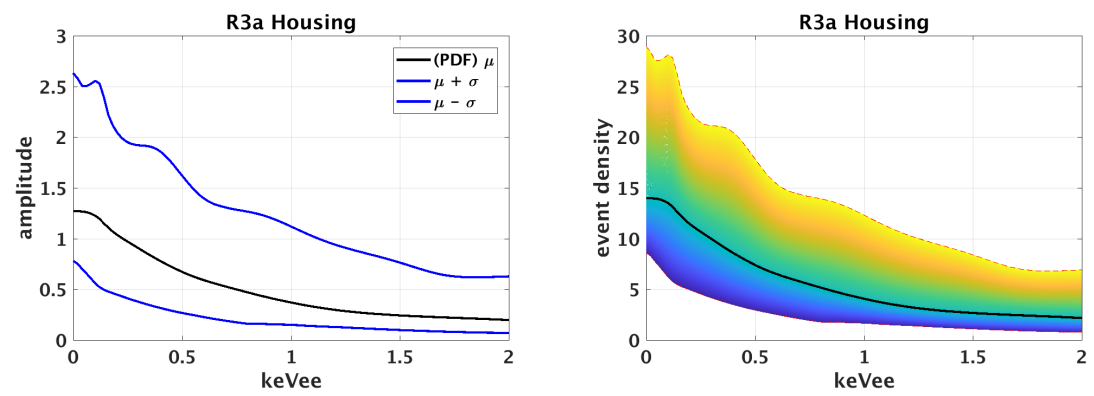

Figure 5.4: (left) Uncertainty on the shape of the housing background. (right) The uncertainty on the shape and normalization of the housing background, as controlled by a single morphing parameter that is given by a single color.

where $m=0$ results in the mean curve, and $m=1$ and $m=-1$ result in the $1 \sigma$ upper and $1 \sigma$ lower curve respectively, and other values of $m$ result in a linear combination of two of the curves. The $\rho_{\text {low }}, \rho_{\text {med }}$, and $\rho_{\text {up }}$ variables are shown in Fig. 5.4 left as the lower, middle, and upper curves. Figure 5.4 right shows the effect of the morphing parameter, where the color legend is yellow: $m=1$, black: $m=0$, blue: $m=-1$. The event density spectra are normalized to the expected number of events for that background (from the normalization based on the alpha count study, discussed in Sec. 5.1.5). For example, for the Ge surface spectrum:

$$
\int \rho_{G e}(E) d E=N_{G e}
$$

While the morphing parameters are designed to keep track of the uncertainty due to the voltage cut and voltage map systematics, we can additionally incorporate the uncertainty on the surface background normalization due to the alpha counts measurement. Incorporating this extra uncertainty has the effect of increasing the uncertainty on the morphing parameters.

From the alpha study, the total number of events we expect, with no cuts applied, from each surface component is $N_{G e}=5.7 \pm 1.5, N_{H S}=11.0 \pm$ $0.68, N_{T L}=28 \pm 3.0$ with correlations $\rho_{G e-H S}=-0.35, \rho_{G e-T L}=-0.18$, $\rho_{H S-T L}=-0.19$. The morphing parameters, because of their connection to an efficiency systematic uncertainty, are $100 \%$ correlated. For example, if the voltage cut from the surface background modeling procedure were to be looser than its approximated central value value by $1 \sigma$, then all the spectra would shift up together. We use Monte Carlo techniques to combine the 


\subsection{Likelihood and Limit Setting}

uncertainty from the alpha study and the morphing parameters, and the resulting matrix describing the covariance between numbers of events from the germanium, housing, and top lid is:

$$
\mathbf{V}=\left(\begin{array}{ccc}
8.9 & 28.6 & 25 \\
28.6 & 149 & 107 \\
25 & 107 & 111
\end{array}\right)
$$

With the uncertainties from the alpha study and the morphing parameters combined into a covariance matrix for the number of surface background events from different sources, we can propagate the correlated uncertainty to the morphing parameters. Following standard error propagation rules, and with variable names $m_{1}=m_{G e}, m_{2}=m_{H S}, m_{3}=m_{T L}$; and $N_{1}=N_{G e}$, $N_{2}=N_{H S}, N_{3}=N_{T L}$ with a covariance matrix $\mathbf{V}$ between the three variables, we can determine the covariance/variance between the variables, for example between $m_{1}$ and $m_{2}$, as:

$$
\mathbf{M}_{m 1, m 2}=\left.\sum_{i, j}^{3} \frac{\partial m_{1}}{\partial N_{i}} \frac{\partial m_{2}}{\partial N_{j}}\right|_{x=\mu} \times V_{i j},
$$

where the relationship between a morphing parameter and a corresponding number of events is known and displayed in Fig. 5.4.

This gives the following covariance matrix between morphing parameters:

$$
\mathbf{M}=\left(\begin{array}{lll}
1.24 & 0.89 & 0.91 \\
0.89 & 1.04 & 0.87 \\
0.91 & 0.87 & 1.06
\end{array}\right)
$$

With the morphing parameters as fit parameters in the likelihood, they are constrained with a three-dimensional Gaussian whose variances/covariances are given by the $\mathbf{M}$ matrix above. Explicitly, the constraint is given by:

$$
\ln \mathcal{L}_{\mathbf{M} \text { Constraint }}=-\frac{1}{2}\left[\sum_{i=1}^{3} \sum_{j=1}^{3} \mathbf{M}_{i j}^{-1}\left(m_{i}-\mu_{m_{i}}\right)\left(m_{j}-\mu_{m_{j}}\right)\right]
$$

where $\mu_{m}$ is the mean of the constraint and $m$ are the fit parameters.

\subsubsection{Likelihood Function}

We employ the unbinned extended maximum likelihood method to fit our signal and background models to the data. The signal and background 


\subsection{Likelihood and Limit Setting}

models discussed above are incorporated as a product of three likelihoods: a term to allow the fitted number of signal and background events to have Poissonian fluctuations around the observed number of events, a term with the signal and background distributions to discriminate between signal and backgrounds, and a term that constrains certain parameters with prior information:

$$
\mathcal{L}=\mathcal{L}_{\text {Poiss. }} \times \mathcal{L}_{\text {energy dist. }} \times \mathcal{L}_{\text {Constr. }} .
$$

The data input into the likelihood are the number of events passing all cuts, as well as the energies of those events. Taking the logarithm of likelihood, and dropping constant terms, which are unnecessary for maximizing the likelihood, we obtain, for the $\mathcal{L}_{\text {Poiss. }}$ and $\mathcal{L}_{\text {energy dist. terms: }}$

$$
\begin{aligned}
\ln \left(\mathcal{L}_{\text {Poiss. }}\right)+\ln \left(\mathcal{L}_{\text {energy dist. }}\right)=-[ & \left.\nu_{\chi}+\sum_{b} \nu_{b}+\sum_{s b} \nu_{s b}\right] \\
& +\sum_{i=1}^{N} \ln \left[\nu_{\chi} f_{\chi}\left(E_{i}, \vec{n}\right)\right. \\
& +\sum_{b} \nu_{b} f_{b}\left(E_{i}, \vec{n}\right) \\
& \left.+\sum_{s b} \rho_{s b}\left(E_{i}, m_{s b}, \vec{n}\right)\right]
\end{aligned}
$$

The $\nu_{\chi}$ is the expected number of events from the WIMP component, $\nu_{s b}$ are expected event numbers from the surface background components, and $\nu_{b}$ are expected event numbers from the non-surface backgrounds. The $f$ terms are PDFs for the energy of the WIMP distribution and the nonsurface background distributions. The $\rho$ terms are the event densities, as a function of energy, of the surface backgrounds, which depend on the morphing parameters $m$. The $\rho$ variables have normalization of events/energy such that if they are integrated over energy the result is a number of events coming from that surface background, as described in Eq. 5.8. Therefore, the $\rho$ terms have the same units as the $\nu_{b} f_{b}$ terms in the same sum. The $\vec{n}$ is a vector of efficiency and resolution parameters that allow the shapes of the $f_{b}$ and $\rho$ shapes to shift to within a prior determined range that is representative of the uncertainty of the efficiency and resolution. The iterator $i$ is over the $\mathrm{N}$ events that are being fitted.

The full constraint term in the likelihood is shown in Eq. 5.15. Because the Lindhard $k$ constraint is in 1 dimension it is instructive to step 


\subsection{Likelihood and Limit Setting}

through that constraint, and then apply analogously the same idea in higher dimensions when thinking about the other constraints. The Lindhard $k$ is a parameter in the fit that can vary in order to maximize the likelihood, but this constraint applies a penalty (a negative contribution to the likelihood value) if $k$ is different than the central value of the prior on $k$. This central value is given by $\mu_{k}=0.157$, as given by Lindhard theory applied to germanium. For the form of the constraint, we use a Gaussian prior. Therefore, the magnitude of the penalty is determined by the uncertainty on $\mu_{k}$, which is given by $\sigma_{k}$, and is estimated from auxiliary measurements of the ionization yield in germanium [73]. Because these measurements do not provide precise information about the NR ionization yield, particularly at low energy, we use a weak constraint on $k$ by choosing $\sigma_{k}=0.05$.

$$
\begin{aligned}
\ln \left(\mathcal{L}_{\text {Constr. }}\right)= & -\frac{\left(k-\mu_{k}\right)^{2}}{2 \sigma_{k}^{2}} \\
& -\frac{1}{2}\left[\sum_{i, j}^{3}\left(e_{i}-\mu_{e_{i}}\right) \mathbf{E}_{i j}^{-1}\left(e_{j}-\mu_{e_{j}}\right)\right] \\
& -\frac{1}{2}\left[\sum_{i, j}^{6}\left(r_{i}-\mu_{r_{i}}\right) \mathbf{R}_{i j}^{-1}\left(r_{j}-\mu_{r_{j}}\right)\right] \\
& -\frac{1}{2}\left[\sum_{i, j}^{3}\left(m_{i}-\mu_{m_{i}}\right) \mathbf{M}_{i j}^{-1}\left(m_{j}-\mu_{m_{j}}\right)\right] .
\end{aligned}
$$

We constrain the three parameters describing the signal efficiency, $\vec{e}$, with a 3D Gaussian prior using the results of Sec. 4.9. The center of the 3D Gaussian is given by the best-fit values of the parameters $\vec{\mu}_{e}$, and its shape is determined by the covariance matrix between best-fit values, given by $\mathbf{E}$. We similarly constrain the resolution parameters, $\vec{r}$, using the 6D Gaussian prior from the resolution model of Sec. 5.1.1, with best-fit resolution model values of $\vec{\mu}_{r}$ and covariance matrix R. Because the Run 3a and Run 3b detector resolutions were modeled independently, $\mathbf{R}$ contains zeros in elements linking the two periods. The morphing parameters, $\vec{m}$, which incorporate systematics of the surface backgrounds, are constrained in the final term of Eq. 5.15. The expected values for the morphing parameters, $\vec{\mu}_{m}$, as well as the covariance matrix $(\mathbf{M})$ between them, determine the constraint. 


\begin{tabular}{l|c|r}
\hline Variable & Constraint & Parameter Boundary \\
\hline $\mathbf{e}$ (efficiency vector) & 3D Gaussian & All variables $>0$ \\
$\mathbf{m}$ (morphing vector) & 3D Gaussian & All event densities $>0$ \\
$\mathbf{r}$ (resolution vector) & 6D Gaussian & All variables $>0$ \\
$k$ (Lindhard) & 1D Gaussian & $0<k<0.3\left(3 \sigma\right.$ from $\left.\mu_{k}\right)$ \\
$\sigma_{\chi}$ (DM cross section) & No Constraint & $\sigma_{\chi}>0$ \\
\hline
\end{tabular}

Table 5.6: Constrained or bound variables in the likelihood fit

\subsubsection{Limit-Setting Approach}

For a given WIMP mass we test the hypothesis that a WIMP signal with cross section $\sigma_{\chi}$ exists in the data. The PLR method allows us to calculate the probability of observing a $\sigma_{\chi}$ signal strength when the hypothesis is true. Then we scan over $\sigma_{\chi}$ to calculate the probability ( $\mathrm{p}$ value) of different signal strength hypotheses, and then quote a cross section sensitivity when the probability of the signal hypothesis equals 0.1 . This is equivalent to quoting a cross section when the signal hypothesis is rejected at the $90 \%$ confidence level (CL).

The first step in the limit setting procedure is to compute the $q$ statistic for the dataset, for a certain signal strength hypothesis, $\sigma_{\chi}$ :

$$
q_{\sigma_{\chi}}= \begin{cases}-2 \ln \lambda\left(\sigma_{\chi}\right) & \hat{\sigma}_{\chi}<\sigma_{\chi} \\ 0 & \hat{\sigma}_{\chi}>\sigma_{\chi}\end{cases}
$$

where $\lambda$ is defined as

$$
\lambda\left(\sigma_{\chi}\right)=\frac{\mathcal{L}\left(\sigma_{\chi}, \hat{\hat{\theta}}\right)}{\mathcal{L}\left(\hat{\sigma}_{\chi}, \hat{\theta}\right)} .
$$

The numerator of $\lambda\left(\sigma_{\chi}\right)$ is the likelihood of a fit that has constrained the signal component to the test hypothesis value $\sigma_{\chi}$, and $\hat{\hat{\theta}}$ are the values of the nuisance parameters that maximize the likelihood given the constraint on $\sigma_{\chi}$. The denominator of $\lambda\left(\sigma_{\chi}\right)$ is the likelihood with no constraints - the cross section $\sigma_{\chi}$ is permitted to float, along with the nuisance parameters, and the values that maximize the likelihood are labeled $\hat{\sigma}_{\chi}$ and $\hat{\theta}$.

To provide some sense for these statistical variables, $\lambda\left(\sigma_{\chi}\right)$ is a number between 0 and 1 , and $\lambda\left(\sigma_{\chi}\right)=1$ corresponds to the most test-signallike outcome - the best fit signal value of $\sigma_{\chi}$ equals the hypothesis value of 


\subsection{Likelihood and Limit Setting}
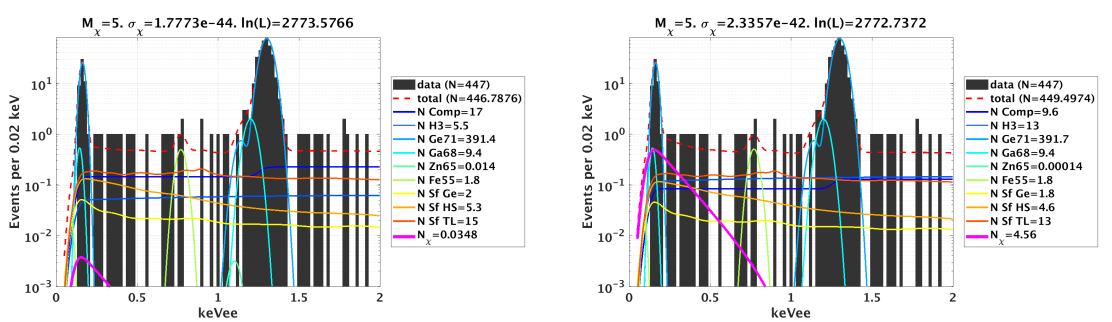

Figure 5.5: (left) Likelihood fit, to a pseudo-dataset, with WIMP cross section freely floating. (right) A likelihood fit, to the same pseudo-dataset, with WIMP cross section clamped to $\sigma_{\chi}=2.3 \times 10^{-42} \mathrm{~cm}^{2}$. On both plots, the best fit number of background events from the different backgrounds are shown in the legend, and the likelihood values are shown in the plot title.

$\sigma_{\chi}$. The $\lambda\left(\sigma_{\chi}\right)=1$ case corresponds to $q_{\sigma_{\chi}}=0$. Signal hypotheses for which $\hat{\sigma}_{\chi}>\sigma_{\chi}$ are compatible with the data when calculating upper limits. Therefore $q_{\sigma_{\chi}}$ is set to 0 in these cases, which is the value that indicates the highest degree of compatibility between the signal hypothesis and the data.

Using this likelihood ratio statistic all parameters in the likelihood other than $\sigma_{\chi}$ (i.e. the systematic uncertainty parameters and the numbers of background events) are profiled out as nuisance parameters by maximizing $\mathcal{L}$ as a function of these parameters with $\sigma_{\chi}$ held constant.

An example of the signal-rejection power of the likelihood ratio test is demonstrated in the following plot which shows fit results of background models and a $5 \mathrm{GeV} / c^{2} \mathrm{DM}$ signal to a pseudo-dataset. The left plot shows a fit representing the denominator of $\lambda\left(\sigma_{\chi}\right)$ with the WIMP cross section floating, giving a $\ln \mathcal{L}$ value of 2773.577. The right plot shows a fit representing the numerator of $\lambda\left(\sigma_{\chi}\right)$ with the WIMP cross section clamped to $2.3 \times 10^{-42} \mathrm{~cm}^{2}$, giving a $\ln \mathcal{L}$ value of 2772.737 . Therefore, $\lambda\left(\sigma_{\chi}\right)=\Delta \ln \mathcal{L}=$ -0.84 and $q_{\sigma_{\chi}}=1.68$. Based on this $q_{\sigma_{\chi}}$ value we can assign a rejection confidence level (CL) to a $5 \mathrm{GeV} / c^{2}$ DM particle with $\sigma_{\chi}=2.3 \times 10^{-42} \mathrm{~cm}^{2}$ existing in this data set. We now discuss how to calculate the rejection CL.

After obtaining the $q_{\sigma_{\chi} \text {,obs }}$ statistic for a data set, the probability of obtaining that $q_{\sigma_{\chi} \text {,obs }}$ value assuming the signal hypothesis is true (i.e. that a signal of size $\sigma_{\chi}$ exists in the data) is given by

$$
p_{\sigma_{\chi}}=\int_{q_{\sigma_{\chi}, \text { obs }}}^{\infty} f\left(q_{\sigma_{\chi}} \mid \sigma_{\chi}\right) d q_{\sigma_{\chi}} .
$$




\subsection{Likelihood and Limit Setting}
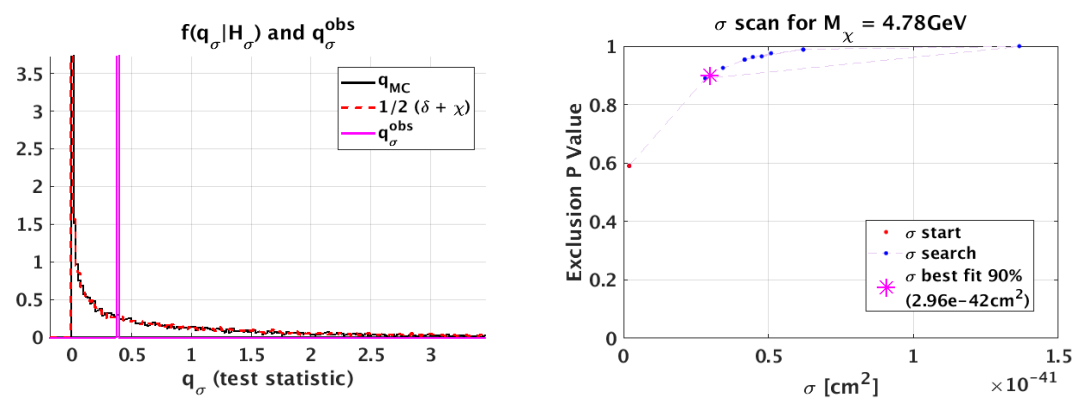

Figure 5.6: An example $q_{\sigma_{\chi}, \text { obs }}$ relative to a MC produced $f\left(q_{\sigma_{\chi}} \mid \sigma_{\chi}\right)$ distribution, as well as the theoretical $(1 / 2) \times\left(\delta(0)+\chi^{2}\right)$ distribution.

Here $f\left(q_{\sigma_{\chi}} \mid \sigma_{\chi}\right)$ is the probability distribution function of $q_{\sigma_{\chi}}$ when the test hypothesis is true. We reject $\sigma_{\chi}$ at $90 \%$ confidence when $p_{\sigma_{\chi}}=0.1$.

Computing $f\left(q_{\sigma_{\chi}} \mid \sigma_{\chi}\right)$ requires significant amounts of computation because the distribution of $q_{\sigma_{\chi}}$ values must be approximated by simulating and fitting at least hundreds of pseudo-datasets containing background + signal components. To avoid calculating $f\left(q_{\sigma_{\chi}} \mid \sigma_{\chi}\right)$, Wilks' theorem [140] says that this distribution asymptotically approaches a mixture of a delta function at zero and a chi-square distribution with one degree of freedom, with the delta function $\delta(0)$ and the $\chi_{\mathrm{dof}=1}^{2}$ distribution each having a weight of $1 / 2$. In the text below we will refer to this mixture of distributions as $(1 / 2) \times\left(\delta(0)+\chi^{2}\right)$. If it can be shown that this asymptotic behavior is realized for the CDMSlite R3 likelihood function then $f\left(q_{\sigma_{\chi}} \mid \sigma_{\chi}\right)=(1 / 2) \times\left(\delta(0)+\chi^{2}\right)$ can be assumed. We calculate $f\left(q_{\sigma_{\chi}} \mid \sigma_{\chi}\right)$ by fitting pseudo-datasets of signal + background and confirm that these distributions converge to the distribution predicted by Wilks' Theorem. One such of these checks is shown in Fig. 5.6, and so we use the theoretical distribution in sensitivity and limit calculations.

\section{The $\mathrm{CL}_{\mathrm{s}}$ Method}

A slight modification is made to the above formalism of setting a 90\% CL limit. This modification is used in order to protect against the possibility of the profile likelihood method excluding a WIMP cross section to which the experiment is not sensitive in the case that the background statistically fluctuates to a low number of events. The method, called the $\mathrm{CL}_{\mathrm{s}}$ method [141], gives a slightly higher signal cross section for the $90 \%$ exclusion than would otherwise be obtained, and in this sense it is conservative. 
The method adds an additional integral into the denominator of the p-value that is the probability of $\mathrm{q}$ to be larger than the observed test statistic under the background-only hypothesis:

$$
p_{\sigma_{\chi}, C L s}=\frac{\int_{q_{\sigma_{\chi}, o b s}}^{\infty} f\left(q_{\sigma_{\chi}} \mid \sigma_{\chi}\right) d q_{\sigma_{\chi}}}{\int_{q_{\sigma_{\chi}, o b s}}^{\infty} f\left(q_{\sigma_{\chi}} \mid \sigma_{\chi}=0\right) d q_{\sigma_{\chi}}}
$$

This has the effect of making $p_{\sigma_{\chi}, C L s}$ greater than $p_{\sigma_{\chi}}$, which will require a higher $\sigma_{\chi}$ hypothesis to be tested in order to reach $p_{\sigma_{\chi}}=0.1$ and exclude that hypothesis at $90 \%$ CL.

\subsection{Results}

The best-fit value of $\sigma_{\chi}$ for the DM masses considered in this analysis is found to be well below the experiment's sensitivity, and so we choose to set an upper limit.

\subsubsection{Background Model Goodness of Fit}

After unsalting the R3 data set, we test whether the background model likelihood is a good fit to the data. The likelihood fitting procedure described in Sec. 5.2 provides no information as to the goodness of fit (GOF) of the model to the data, and therefore we define a procedure to evaluate the GOF that outputs a probability (i.e. a p-value) for the data on the assumption that the model is correct. We use the Cramér-von Mises GOF statistic [142] because it does not require binning of the data, overcomes some deficiencies of the more common KS test, yet is still relatively simple compared to some alternative GOF metrics. The Cramér-von Mises statistic $C V M$ is defined as:

$$
C V M=n \int\left[F_{n}(x)-F(x)\right]^{2} P(x) d x
$$

where $F_{n}(x)$ is the empirical cumulative distribution function (CDF) of the data, $F(x)$ is the CDF of the model's fit to the data, and $P(x)$ is the PDF of the model's fit to the data. The $n$ variable is the number of data points. Also, since our model is a function of energy, the $x$ variable is energy. A larger Cramér-von Mises statistics corresponds to a worse fit. 
We use Monte Carlo to calculate the confidence level that the Cramérvon Mises statistic indicates that the data comes from the background model. MC distributions of the goodness-of-fit statistic are calculated using fits to fake data sets that have been generated according to the background model. This custom MC approach allows us to take into account the systematic uncertainties in the background model. The steps of the procedure are:

1. Fit the real data and determine best fit values and posterior distributions for the model parameters.

(a) This fit and the posterior distributions are shown in Fig. 5.10.

2. Calculate the Cramér-von Mises statistic for the fit to the real data.

3. Generate 1000 pseudo-experiments based off the fit to the real data.

(a) For the number of events from each background, sample from a Poissonian distribution with mean centered at the best fit values from step (1).

(b) For the other nuisance parameters, sample from the posterior distributions from step (1).

(c) Fit and calculate the Cramér-von Mises statistic for each pseudoexperiment

4. Calculate the fraction of the pseudo-experiments' Cramér-von Mises statistics that are larger than the one for the real data from step (2). This fraction is the p-value.

Prior to unblinding, we agreed on a p-value threshold of 0.05 , below which we would investigate inaccuracies in the background model, abandon the limit obtained with the profile likelihood method, and resort to the more conservative optimum interval limit-setting technique.

As shown in Fig. 5.7, the GOF procedure gives a p-value of 0.988 for the hypothesis that the data comes from the background model, indicating a particularly good fit. Two varieties of checks were performed to ensure that the above procedure was unbiased. First, the procedure above was repeated but removing step (3b) and instead, when creating the pseudodatasets, setting the systematic nuisance parameters to the best fit values we found in step (1). This changed the p-value only slightly, from 0.988 to 0.986 , indicating that the high p-value is not a result of an overestimation of 


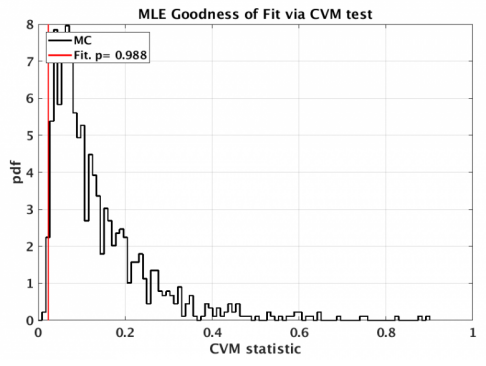

Figure 5.7: Cramér-von Mises Statistic of the fit to the data (red line) and 1000 pseudo experiments.

our systematic uncertainties. Second, pseudo-experiments themselves were tested as if they were the real data set (i.e. they were fit with the likelihood and the Cramér-von Mises statistic was determined for that fit, and subsequently a set of pseudo-experiment based on that fit were created and fit, etc.). This procedure, carried out 7 times, did not show any bias in the obtained p-value scores, which were between 0.053 and 0.85 .

\subsubsection{Exposure of Salt}

With the analysis (cuts, background models, likelihood function) frozen, the dataset was unblinded by exposing the salt in the dataset. Followup checks were performed on the salt and confirmed that it was inserted at the intended magnitude and with the intended energy and radial distribution, as shown in Fig. 5.8.

The passage fraction of the salt as a function of energy from $0-2 \mathrm{keV}_{\text {ee }}$ was examined to check the expectation that the salt passage fraction matched, to within uncertainties, the estimated signal efficiency. The red error bars in Fig. 5.9 left show the salt passage fraction with all cuts (DQ efficiency cuts + multiples + muon veto + radial $)$ applied. Relative to the estimated signal efficiency, the passage fraction is for salt is systematically lower. The multiples cut is the largest reason for the reduced salt passage fraction. The multiples cut and muon veto cut passage fraction of the salt is the largest discrepancy with our estimated signal efficiency, since both of these cuts were estimated to be $>98 \%$ efficient for signal, while they are $\sim 80 \%$ efficient for salt.

The reason why the multiples cut has a lower passage fraction of salt than we estimated for the signal is that the replaced events come from background 

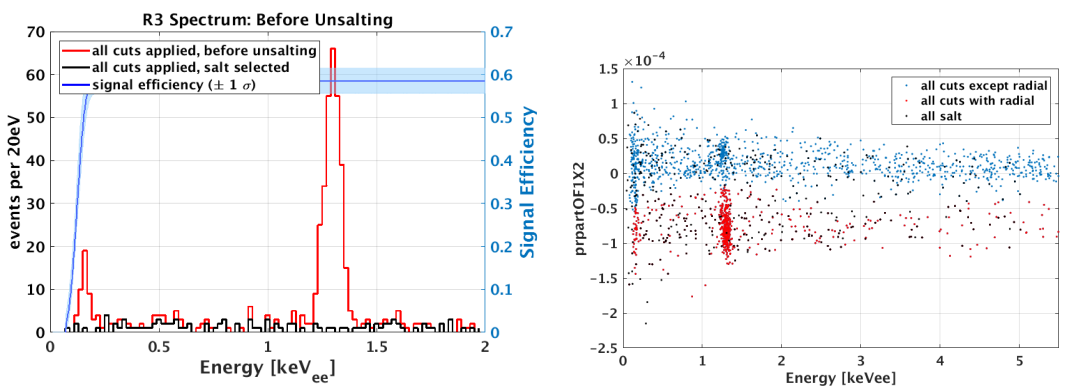

Figure 5.8: (left) The R3 final spectrum with all cuts applied, and with salt included and highlighted). (right) The distribution of salt (with no cuts applied) highlighted in the energy vs. $\xi$ radial parameter plane, with the real data shown in the background.

(i.e. $\alpha, \beta, \gamma$ recoils), and these sources are expected to pass the multiples cut with lower efficiency than a DM signal. This is explicitly shown in Fig. 5.9 center, where the unsalted dataset is loaded and the events that were replaced by salt are selected. $73 / 393 \simeq 19 \%$ of these events are identified by the multiples cut. When the waveform of these events is replaced with a salted waveform (i.e. a good low energy pulse) the event is still likely going to be identified as a multiple because the waveform information on the other detectors is not changed in the salting algorithm. This $19 \%$ multiples rate is consistent with Fig. 5.9 left.

Figure 5.9 right shows the salt passage fraction with just the DQ efficiency cuts and radial cut applied to the salt in pink. Omitting the multiples and muon veto cuts removes the systematic $1.5-2 \sigma$ disagreements. This is a confirmation that the salting procedure was successful, as it assumed a distribution in event ntuple parameter space that resembled a DM signal.

\subsubsection{Final Spectrum Fit}

The final Run 3 spectrum after application of all selection cuts is shown in Fig. 5.10. The main features are the ${ }^{71} \mathrm{Ge}$ electron-capture $L$ - and $M$ shell peaks at 1.30 and $0.16 \mathrm{keV}_{\text {ee }}$ respectively. Events contributed from backgrounds other than ${ }^{71} \mathrm{Ge}$ exist between the peaks and are well modeled. We do not observe a population of events below the $M$ shell, which is consistent with the steep decrease of the signal efficiency in this range and consistent with the expectations from the background model. Posteriors for the number of events contributing each background are shown in 


\subsection{Results}
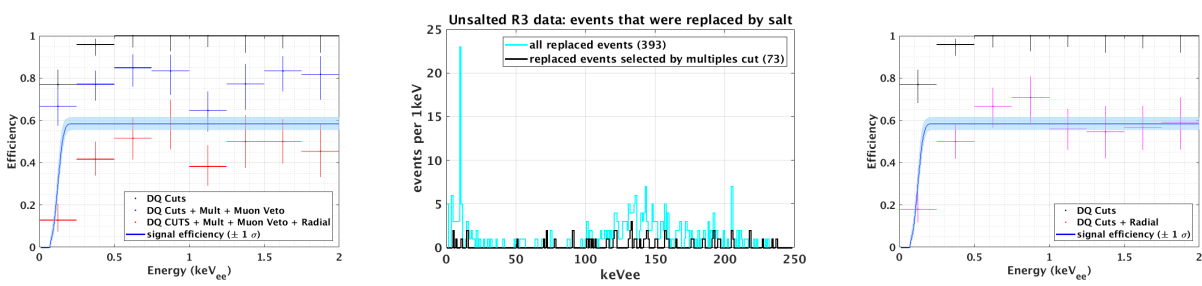

Figure 5.9: (left) The salt passage fraction with sequential application of cuts (shown in the legend) compared to the signal efficiency estimate. (center) The energy spectrum of events, in the unsalted dataset, selected to be replaced by salt events. Roughly $19 \%$ of them were multiples, consistent with the passage fraction given by the left hand figure. (right) The salt passage fraction, but without the multiples and muon veto cut.

Fig. 5.10 right. In the figure axes labels, m1, m2, m3 corresponds to the morphing parameters for the Ge, Housing, and Top Lid surface backgrounds. As expected, a strong covariance is observed between the Compton and ${ }^{3} \mathrm{H}$ background components, which in this energy range do not contain sufficiently distinct spectral features to remove their degeneracy in the fit. The surface background components are strongly correlated through the prior constraint covariance matrix, $\mathbf{M}$, described in Sec. 5.2.

We find that the surface background component covariances from the likelihood fit match the prior constraint covariances, shown in the top row of Fig. 5.11, indicating that these $0.07-2.0 \mathrm{keV}_{\text {ee }}$ data do not provide any additional information on the surface background. Figure 5.11 also shows that all of the systematic uncertainty parameters that were constrained in the likelihood (i.e. resolution and efficiency parameters) are within the range to which they were constrained by the prior. Additionally, the posteriors have similar widths as the priors indicating that the data are not providing additional information for these parameters.

\subsubsection{Background Rates}

We calculate the average background rates of single-scatter events between the ${ }^{71}$ Ge peaks, corrected for efficiency, as shown in Table 5.7. The Run 3 background rate is higher than Run 2, in line with the expectation of the higher surface background for an endcap detector (T2Z1) relative to T5Z2. T5Z2 was surrounded on both of its faces by the two adjacent detectors, whereas T2Z1 was the highest detector in the tower and therefore had one 

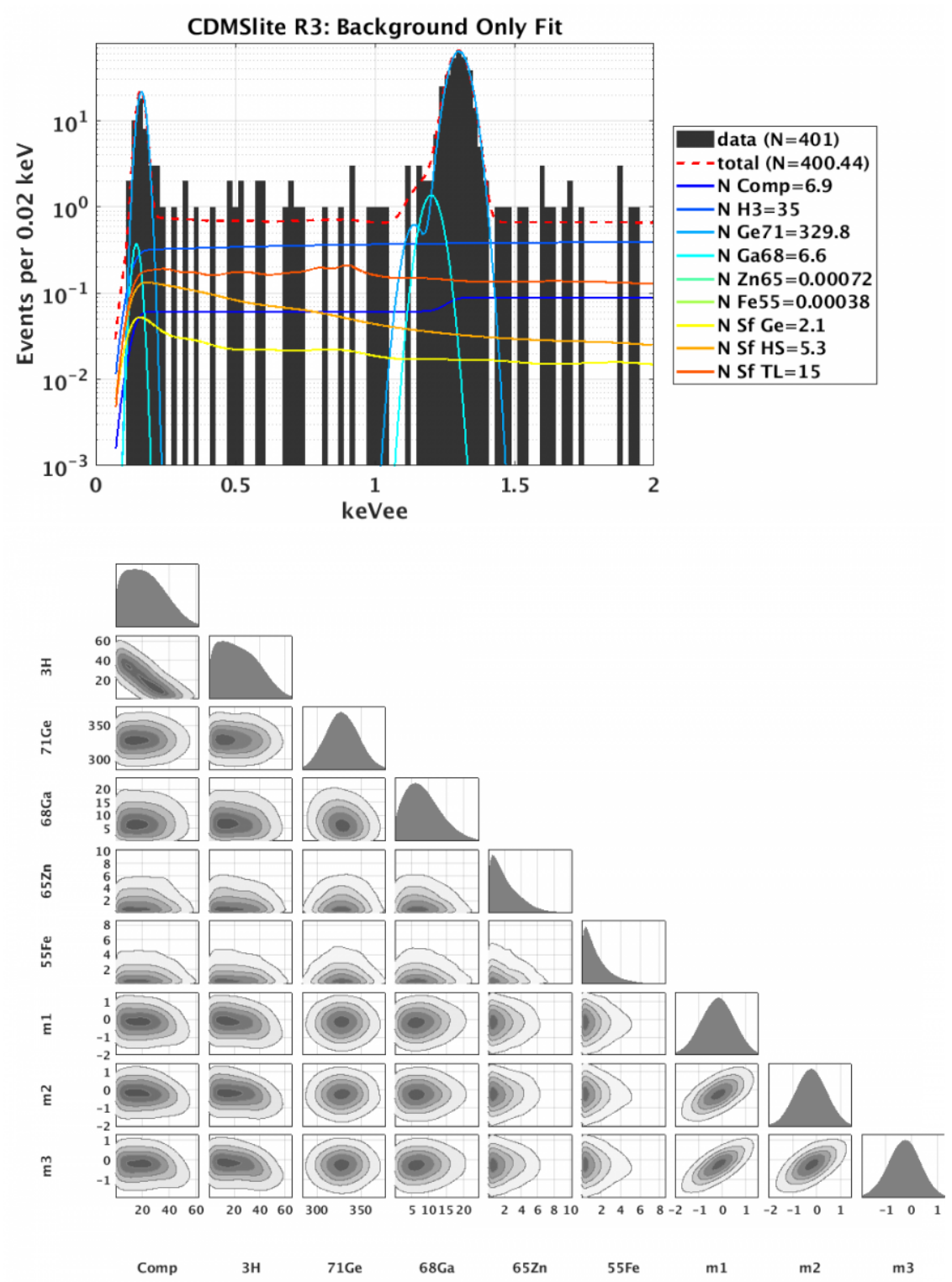

Figure 5.10: (top) Background best fit amplitudes overlaid on the Run 3 unsalted data. "Sf" is an abbreviation for "surface". (bottom) $1 \mathrm{D}$ and 2D posterior distributions for the number of events (or morphing parameter in the case of surface backgrounds) contributing from each background. By posterior, we do not mean to imply that these are the results of a Bayesian analysis; rather, these are the fit results from the likelihood, with asymmetric uncertainties determined with a Markov-chain Monte Carlo sampling algorithm. 


\subsection{Results}
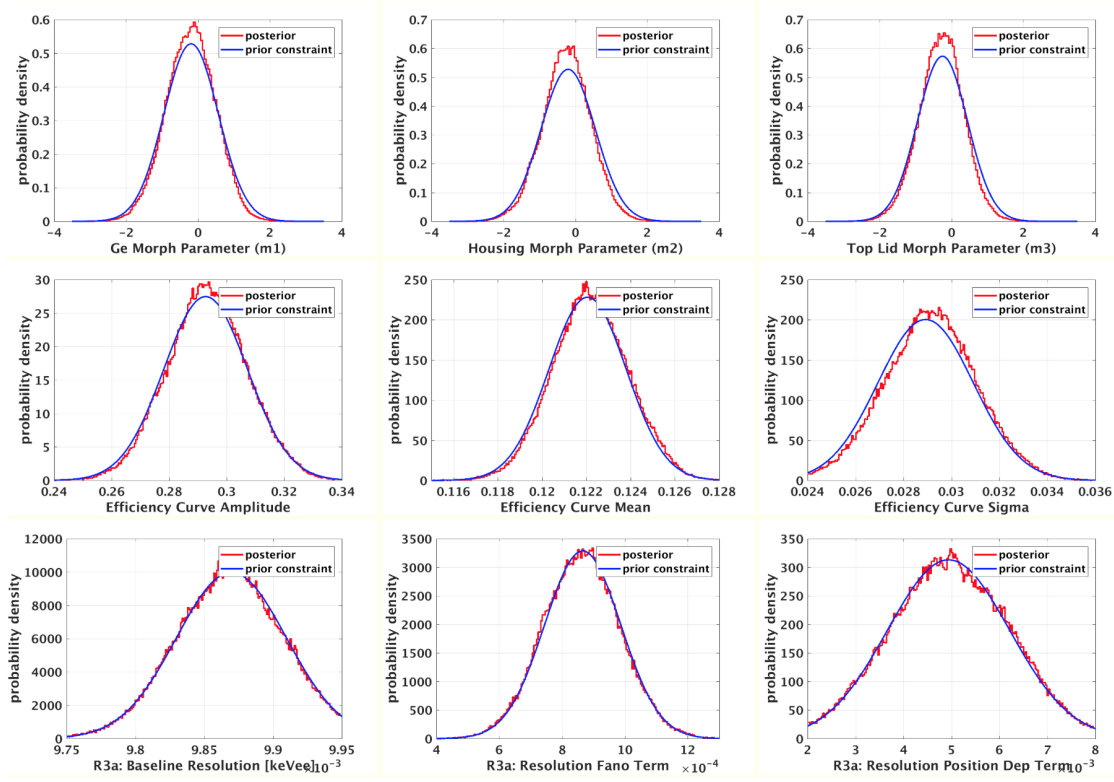

Figure 5.11: Comparing prior constraints with fit results of the maximum likelihood for the efficiency, morphing, and resolution parameters. The similar variance of the prior and posteriors indicates that the data are not providing additional information for these systematic uncertainty parameters, and the similar mean indicates that our signal efficiency and resolution model parameters are in agreement with the data. 


\subsection{Results}

\begin{tabular}{ccc}
\hline \hline $\begin{array}{c}\text { Range } \\
{\left[\mathrm{keV}_{\mathrm{ee}}\right]}\end{array}$ & $\begin{array}{c}\text { Run 2 Rate } \\
{\left[\mathrm{keV}_{\mathrm{ee} \mathrm{kg} \mathrm{d}}\right]^{-1}}\end{array}$ & $\begin{array}{c}\text { Run 3 Rate } \\
{\left[\mathrm{keV}_{\mathrm{ee}} \mathrm{kg} \mathrm{d}\right]^{-1}}\end{array}$ \\
\hline $0.2-1.2$ & $1.09 \pm 0.18$ & $1.9 \pm 0.3$ \\
$1.4-10$ & $1.00 \pm 0.06$ & $1.3 \pm 0.1$ \\
$11-20$ & $0.30 \pm 0.03$ & $0.71 \pm 0.07$ \\
\hline \hline
\end{tabular}

Table 5.7: Average single-scatter event rates for energy regions between the activation lines in Run 2 and Run 3, corrected for efficiency. All errors contain $\pm \sqrt{N}$ Poissonian uncertainties, and the lowest energy range values additionally include uncertainty from the signal efficiency.

face exposed to the top lid copper. It is also expected from T2Z1's position in the tower that the ability to identify and remove multiple scatters is diminished. Additionally, Run 3 had fewer other active detectors in the full array relative to Run 2. Since the active detectors are used to identify the multiple scatter events, this factor would also reduce the efficiency of the Run 3 multiples cut. Therefore, a higher fraction of multiple scatter events could be passing the multiples cut and contributing to the event counts in the table.

\subsubsection{Sensitivity Estimate}

Prior to unsalting the data, we calculated the 90\% CL sensitivity of the Run 3 analysis to a DM signal based on projected background rates in this analysis's energy region of interest (ROI), 0.07-2.0 keV ee. The sensitivity calculation also uses the likelihood framework presented in Sec. 5.2. To estimate the background rates in the ROI, we measure them in the $5-25 \mathrm{keV}_{\text {ee }}$ range and extrapolate the rates to lower energy. We choose $5 \mathrm{keV}_{\text {ee }}$ because salt was not inserted above this energy and because the DM signal contribution above this energy for DM masses $<10 \mathrm{GeV} / c^{2}$ is expected to be negligible. Also, because $5 \mathrm{keV}_{\text {ee }}$ is below the lowest $K$-shell energy of the EC isotopes considered, all background components are constrained in this range. We perform a maximum likelihood fit, using the likelihood defined in Eq. 5.13 but without the DM signal. We also omit the resolution and efficiency systematic uncertainties because those extra terms are unnecessary when fitting the $5-25 \mathrm{keV}_{\text {ee }}$ background spectrum. This fit provides best-fit values of, as well as covariances between, background rates in the $5-25 \mathrm{keV}_{\text {ee }}$ range for the nine background components. The expected background in the ROI can directly be calculated from the best fit in the $5-25 \mathrm{keV}_{\text {ee }}$ range. 
The uncertainty is determined from the covariance matrix of the fit.

Background-only pseudo-experiments are then generated by sampling from the nine different background distributions. The number of events thrown for each background component is randomized, first by sampling from the 9D Gaussian distribution provided by the $5-25 \mathrm{keV}_{\text {ee }}$ maximum likelihood fit and second by adding a Poissonian fluctuation to the sampled value. The $90 \%$ CL PLR limit, using the $\mathrm{CL}_{s}$ technique, is calculated for 500 of these pseudo-experiments, and the resulting $\pm 1 \sigma$ bands are shown as the red band in Fig. 5.12.

In addition to determining parameters for generating the pseudo-experiments, the $5-25 \mathrm{keV}_{\text {ee }}$ fit provides constraints on the surface background morphing parameters (the $\mu_{m_{i}}$ of Eq. 5.15). While this fit used a prior constraint centered at 0 for all morphing parameters, the respective posteriors peaked at $-0.19,-0.2$, and -0.25 for the germanium, top lid, and housing surface background locations respectively. This indicates a slightly lower surface background rate than predicted by the alpha decay study. The $5-25 \mathrm{keV}_{\text {ee }}$ fit also slightly reduced the uncertainty on the morphing parameters, as given in the following covariance matrix:

$$
\mathbf{M}=\left(\begin{array}{lll}
0.570 & 0.419 & 0.420 \\
0.419 & 0.572 & 0.389 \\
0.420 & 0.389 & 0.485
\end{array}\right)
$$

These updated central values for the constraint were used in the likelihood for both the sensitivity estimate and the final limit, along with the updated covariance matrix for the morphing parameters.

\subsubsection{DM Limit}

Figure 5.13 shows the final CDMSlite Run 3 limit calculated with the spectrum in Fig. 5.10. From $2.5-10 \mathrm{GeV} / c^{2}$ we find a factor of $2-3$ improvement in the excluded DM-nucleon cross section over the CDMSlite Run 2 optimum interval analysis [143]. This improvement is achieved despite the smaller exposure (36 vs. $70 \mathrm{~kg}$-days) and higher background rate in Run 3, demonstrating the discrimination power of the PLR method. Below $2.5 \mathrm{GeV} / c^{2}$, we exclude little to no additional parameter space because the effective energy threshold for this analysis is slightly higher than that for CDMSlite Run 2. 


\subsection{Results}

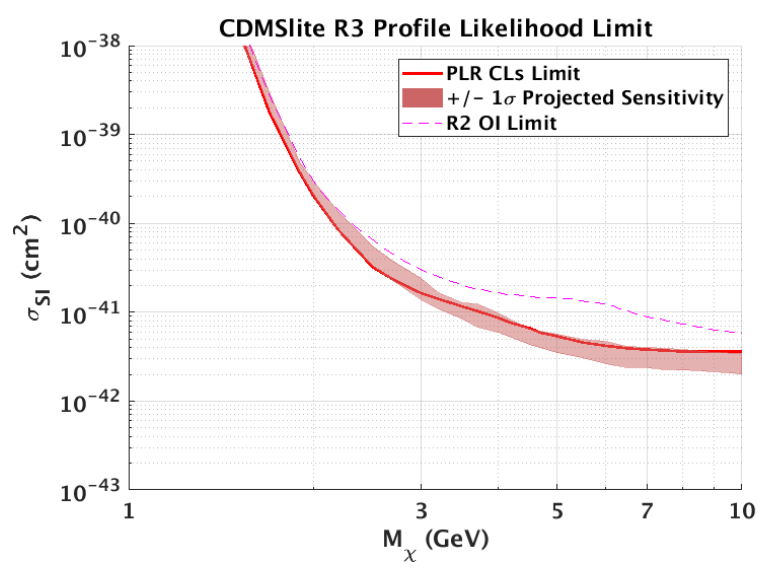

Figure 5.12: (left) R3 $1 \sigma$ sensitivity band and PLR limit (with R2 OI limit overlaid)

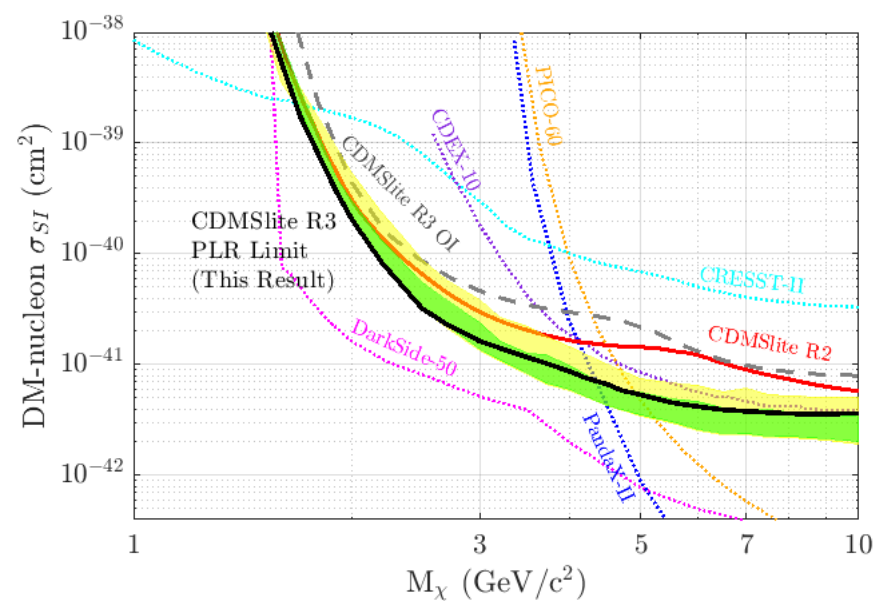

Figure 5.13: The CDMSlite Run 3 90\% CL PLR limit (this result, solid black) on the spin-independent WIMP-nucleon cross section, along with the $\pm 1 \sigma$ and $\pm 2 \sigma$ sensitivity bands (green and yellow respectively). The CDMSlite Run 3 optimum interval limit (dashed grey) and Run 2 (red) optimum interval limit [143] are overlaid. Examples of limits from other detector technologies are overlaid: DarkSide-50 2018 No Quenching Fluctuations (magenta) [84]; PandaX-II 2016 (blue) [43]; PICO-60 2017 (orange) [144]; CRESST-II 2016 (cyan) [94]; CDEX-10 2018 (purple) [145]. 


\section{Chapter 6}

\section{Conclusion and Future Outlook}

The ability of the CDMSlite operating mode to improve the SuperCDMS detectors' sensitivity to low mass DM is clear from the exclusion limits in Fig. 5.13. The CDMSlite Run 1 result first demonstrated the promise of the Neganov-Trofimov-Luke (NTL) effect to lower the detector threshold, while CDMSlite Run 2 used an improved understanding of the detector noise environment and electric field to reduce backgrounds and further lower the detector threshold, producing world-leading sensitivity to DM particles between 2 and $6 \mathrm{GeV} / c^{2}$. Then in this thesis, the CDMSlite approach was taken a step further.

In Chapter 4 and Chapter 5, we introduced new analysis methods that moved CDMSlite from an exclusion-only analysis to one with discovery potential, in addition to improving the low-mass sensitivity of CDMSlite. Specifically, the results demonstrate successful modeling of radioactive backgrounds in CDMSlite detectors down to low energies, as well as the discrimination power of a profile likelihood fit to set strong limits on a DM signal even in the presence of irreducible backgrounds. A number of analysis developments enabled this approach, including improved rejection of instrumental backgrounds using detector-detector correlations in a boosted decision tree, removal of events at high radii with misreconstructed energies by an improved fiducial volume cut, and Monte Carlo modeling of surface backgrounds in the detector.

The SuperCDMS collaboration is currently constructing a new experiment, SuperCDMS SNOLAB, which will use the NTL effect to reach lower thresholds in detectors designed specifically for high-voltage operation [97, 146]. The analysis developments in the CDMSlite Run 3 analysis, in particular the profile likelihood approach, are planned for future SNOLAB analyses. Because our use of the profile likelihood method for CDMSlite Run 3 was the first time it had been used in a search for DM in a CDMSlite detector, our results show that employment of such a method is possible even when the analysis is pushing to the limits of the detector threshold. 
In addition to the CDMSlite Run 3 analysis, the detector characterization results presented in Chapter 3 provided important information for the projected sensitivity of the SuperCDMS SNOLAB detectors. Namely, the improved baseline resolution measured on prototype lower transition temperature $\left(T_{c}\right)$ detectors confirmed resolution extrapolations for the lower $T_{c}$ detectors being fabricated for SuperCDMS SNOLAB. The number of detectors, as well as their projected resolution, is given in Table 6.1.

The projected DM sensitivity of the SuperCDMS SNOLAB experiment can be calculated using the values from Table 6.1 as well as the background rate projections detailed in Ref. [97]. By moving to the deeper laboratory at SNOLAB, the flux from cosmogenic muons and cosmogenically produced neutrons will be reduced by approximately 2.5 orders of magnitude relative to Soudan. The neutron background is not expected to be significant relative to electron recoil backgrounds, and the decays from ${ }^{3} \mathrm{H}$ are expected to contribute the highest rate in Ge detectors. The projected sensitivities, calculated using the conservative optimum interval method, are shown in Fig. 6.1.

Looking towards the future of DM direct detection, the dotted yellow line in Fig. 6.1 is the region of parameter space where the solar neutrino coherent elastic scatter rate is expected to begin to mask the interaction rate from DM particles. These nuclear recoil events would look like DM events. Of course it is possible that particle DM is discovered before this coherent neutrino scattering "floor" is reached, but if it is not then the coherent neutrino scattering will constitute a new, difficult background to discriminate against. Pushing down to this floor will mark an important benchmark for direct detection, and pushing past it will require innovative new detector designs. 
Chapter 6. Conclusion and Future Outlook

\begin{tabular}{l|cc|cc}
\hline & Ge (iZIP) & Si (iZIP) & Ge (HV) & Si (HV) \\
\hline Number of detectors & 10 & 2 & 8 & 4 \\
Total exposure $(\mathrm{kg} \cdot \mathrm{yr})$ & 56 & 4.8 & 44 & 9.6 \\
Phonon resolution $(\mathrm{eV})$ & 50 & 25 & 10 & 5 \\
Ionization resolution $(\mathrm{eV})$ & 100 & 110 & - & - \\
Voltage Bias (V) & 6 & 8 & 100 & 100 \\
\hline
\end{tabular}

Table 6.1: The projected exposures and detector parameters for the four types of SNOLAB detectors: Ge iZIP, Si iZIP, Ge HV, Si HV. The exposures are based on 5 years of operation with $80 \%$ live time. The HV detectors do not have ionization sensors. The arrangement will be 4 towers of 6 detectors each. Table reproduced from Ref. [97].

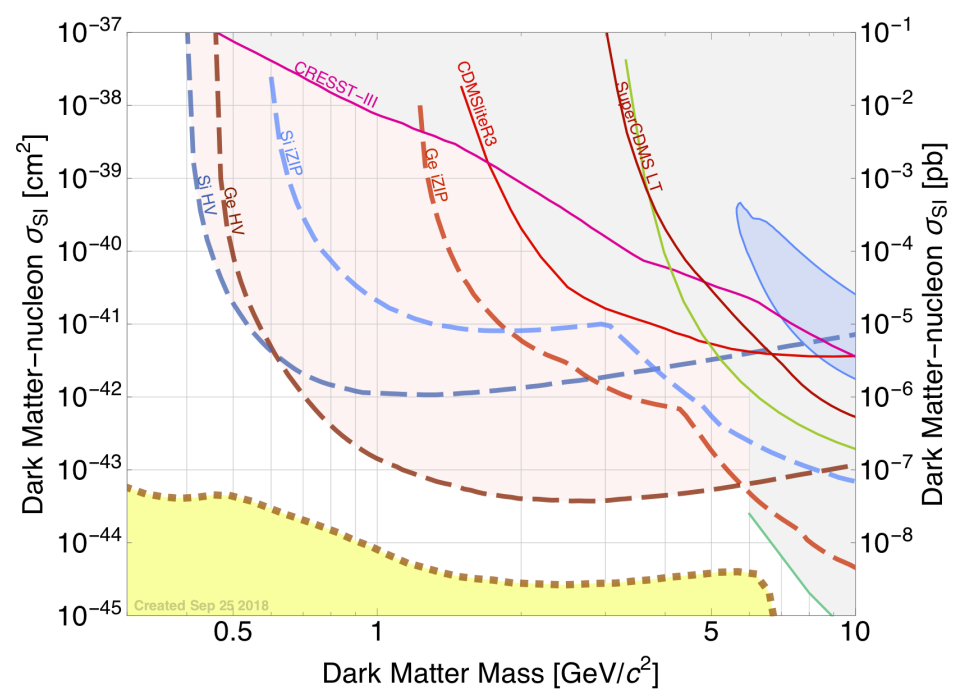

Figure 6.1: The dashed lines show the projected $90 \%$ exclusion sensitivity of the SuperSCDMS SNOLAB experiment, calculated using the optimum interval method. The y axis is the spin-independent WIMP-nucleon cross section. The solid lines show the current $90 \%$ from the CRESST-II [94], SuperCDMS [126, 143], and LUX [147] experiments. The dotted yellow line is the region of parameter space where the solar neutrino coherent elastic scatter rate begins to mask the interaction rate from DM particles, as calculated in Ref. [148]. Figure from SuperCDMS approved public plots. 


\section{Bibliography}

[1] E. Hubble and M. L. Humason. The Velocity-Distance Relation among Extra-Galactic Nebulae. apj, 74:43, July 1931. doi: 10.1086/143323.

[2] F. Zwicky. On the Masses of Nebulae and of Clusters of Nebulae. apj, 86:217, October 1937. doi: 10.1086/143864.

[3] S. Smith. The Mass of the Virgo Cluster. apj, 83:23, January 1936. doi: $10.1086 / 143697$.

[4] D. Hooper G. Bertone. A History of Dark Matter. 2016. URL https: //arxiv.org/pdf/1605.04909.pdf.

[5] D. H. Rogstad and G. S. Shostak. Gross Properties of Five Scd Galaxies as Determined from 21-CENTIMETER Observations. apj, 176:315, September 1972. doi: 10.1086/151636.

[6] H. W. Babcock. The rotation of the Andromeda Nebula. Lick Observatory Bulletin, 19:41-51, 1939. doi: 10.5479/ADS/bib/1939LicOB. 19.41B.

[7] V. C. Rubin and W. K. Ford, Jr. Rotation of the Andromeda Nebula from a Spectroscopic Survey of Emission Regions. , 159:379, February 1970. doi: 10.1086/150317.

[8] V. C. Rubin, W. K. Ford, Jr., and N. Thonnard. Extended rotation curves of high-luminosity spiral galaxies. IV - Systematic dynamical properties, SA through SC. apjl, 225:L107-L111, November 1978. doi: $10.1086 / 182804$.

[9] A. Vikhlinin, A. V. Kravtsov, R. A. Burenin, H. Ebeling, W. R. Forman, A. Hornstrup, C. Jones, S. S. Murray, D. Nagai, H. Quintana, and A. Voevodkin. Chandra cluster cosmology project iii: Cosmological parameter constraints. The Astrophysical Journal, 692(2):1060, 2009. URL http://stacks.iop.org/0004-637X/692/i=2/a=1060. 
[10] J.-P. Kneib, R. S. Ellis, I. Smail, W. J. Couch, and R. M. Sharples. Hubble Space Telescope Observations of the Lensing Cluster Abell 2218. apj, 471:643, November 1996. doi: 10.1086/177995.

[11] Maxim Markevitch. Chandra observation of the most interesting cluster in the universe. 2005. [ESA Spec. Publ.604,723(2006)].

[12] Douglas Clowe, Marua Brada, Anthony H. Gonzalez, Maxim Markevitch, Scott W. Randall, Christine Jones, and Dennis Zaritsky. A direct empirical proof of the existence of dark matter. The Astrophysical Journal Letters, 648(2):L109, 2006. URL http://stacks.iop. org $/ 1538-4357 / 648 / i=2 / a=L 109$.

[13] S. Burles, K. M. Nollett, and Michael S. Turner. Deuterium and big bang nucleosynthesis. Nucl. Phys., A663:861c-864c, 2000. doi: 10.1016/S0375-9474(99)00732-0.

[14] C. Patrignani et al. Review of Particle Physics. Chin. Phys., C40(10): 100001, 2016. doi: 10.1088/1674-1137/40/10/100001.

[15] P. A. R. Ade et al. Planck 2013 results. XVI. Cosmological parameters. Astron. Astrophys., 571:A16, 2014. doi: 10.1051/0004-6361/ 201321591.

[16] S. Dodelson. Modern Cosmology. Elsevier, 2003.

[17] Planck Collaboration, Ade, P. A. R., Aghanim, N., Arnaud, M., Ashdown, M., Aumont, J., Baccigalupi, C., Banday, A. J., Barreiro, R. B., Bartlett, J. G., Bartolo, N., Battaner, E., Battye, R., Benabed, K., Benoît, A., Benoit-Lévy, A., Bernard, J.-P., Bersanelli, M., Bielewicz, P., Bock, J. J., Bonaldi, A., Bonavera, L., Bond, J. R., Borrill, J., Bouchet, F. R., Boulanger, F., Bucher, M., Burigana, C., Butler, R. C., Calabrese, E., Cardoso, J.-F., Catalano, A., Challinor, A., Chamballu, A., Chary, R.-R., Chiang, H. C., Chluba, J., Christensen, P. R., Church, S., Clements, D. L., Colombi, S., Colombo, L. P. L., Combet, C., Coulais, A., Crill, B. P., Curto, A., Cuttaia, F., Danese, L., Davies, R. D., Davis, R. J., de Bernardis, P., de Rosa, A., de Zotti, G., Delabrouille, J., Désert, F.-X., Di Valentino, E., Dickinson, C., Diego, J. M., Dolag, K., Dole, H., Donzelli, S., Doré, O., Douspis, M., Ducout, A., Dunkley, J., Dupac, X., Efstathiou, G., Elsner, F., Enßlin, T. A., Eriksen, H. K., Farhang, M., Fergusson, J., Finelli, F., Forni, O., Frailis, M., Fraisse, A. A., Franceschi, E., Frejsel, A., Galeotta, 
S., Galli, S., Ganga, K., Gauthier, C., Gerbino, M., Ghosh, T., Giard, M., Giraud-Héraud, Y., Giusarma, E., Gjerløw, E., González-Nuevo, J., Górski, K. M., Gratton, S., Gregorio, A., Gruppuso, A., Gudmundsson, J. E., Hamann, J., Hansen, F. K., Hanson, D., Harrison, D. L., Helou, G., Henrot-Versillé, S., Hernández-Monteagudo, C., Herranz, D., Hildebrandt, S. R., Hivon, E., Hobson, M., Holmes, W. A., Hornstrup, A., Hovest, W., Huang, Z., Huffenberger, K. M., Hurier, G., Jaffe, A. H., Jaffe, T. R., Jones, W. C., Juvela, M., Keihänen, E., Keskitalo, R., Kisner, T. S., Kneissl, R., Knoche, J., Knox, L., Kunz, M., Kurki-Suonio, H., Lagache, G., Lähteenmäki, A., Lamarre, J.-M., Lasenby, A., Lattanzi, M., Lawrence, C. R., Leahy, J. P., Leonardi, R., Lesgourgues, J., Levrier, F., Lewis, A., Liguori, M., Lilje, P. B., Linden-Vørnle, M., López-Caniego, M., Lubin, P. M., Macías-Pérez, J. F., Maggio, G., Maino, D., Mandolesi, N., Mangilli, A., Marchini, A., Maris, M., Martin, P. G., Martinelli, M., Martínez-González, E., Masi, S., Matarrese, S., McGehee, P., Meinhold, P. R., Melchiorri, A., Melin, J.-B., Mendes, L., Mennella, A., Migliaccio, M., Millea, M., Mitra, S., Miville-Deschênes, M.-A., Moneti, A., Montier, L., Morgante, G., Mortlock, D., Moss, A., Munshi, D., Murphy, J. A., Naselsky, P., Nati, F., Natoli, P., Netterfield, C. B., Nørgaard-Nielsen, H. U., Noviello, F., Novikov, D., Novikov, I., Oxborrow, C. A., Paci, F., Pagano, L., Pajot, F., Paladini, R., Paoletti, D., Partridge, B., Pasian, F., Patanchon, G., Pearson, T. J., Perdereau, O., Perotto, L., Perrotta, F., Pettorino, V., Piacentini, F., Piat, M., Pierpaoli, E., Pietrobon, D., Plaszczynski, S., Pointecouteau, E., Polenta, G., Popa, L., Pratt, G. W., Prézeau, G., Prunet, S., Puget, J.-L., Rachen, J. P., Reach, W. T., Rebolo, R., Reinecke, M., Remazeilles, M., Renault, C., Renzi, A., Ristorcelli, I., Rocha, G., Rosset, C., Rossetti, M., Roudier, G., Rouillé d’Orfeuil, B., Rowan-Robinson, M., Rubiño-Martín, J. A., Rusholme, B., Said, N., Salvatelli, V., Salvati, L., Sandri, M., Santos, D., Savelainen, M., Savini, G., Scott, D., Seiffert, M. D., Serra, P., Shellard, E. P. S., Spencer, L. D., Spinelli, M., Stolyarov, V., Stompor, R., Sudiwala, R., Sunyaev, R., Sutton, D., Suur-Uski, A.-S., Sygnet, J.-F., Tauber, J. A., Terenzi, L., Toffolatti, L., Tomasi, M., Tristram, M., Trombetti, T., Tucci, M., Tuovinen, J., Türler, M., Umana, G., Valenziano, L., Valiviita, J., Van Tent, F., Vielva, P., Villa, F., Wade, L. A., Wandelt, B. D., Wehus, I. K., White, M., White, S. D. M., Wilkinson, A., Yvon, D., Zacchei, A., and Zonca, A. Planck 2015 results - xiii. cosmological parameters. A\&A, 594:A13, 2016. doi: 10.1051/0004-6361/201525830. URL https://doi.org/10.1051/0004-6361/201525830. 
[18] C. Alcock, R. A. Allsman, D. R. Alves, T. S. Axelrod, A. C. Becker, D. P. Bennett, K. H. Cook, N. Dalal, A. J. Drake, K. C. Freeman, M. Geha, K. Griest, M. J. Lehner, S. L. Marshall, D. Minniti, C. A. Nelson, B. A. Peterson, P. Popowski, M. R. Pratt, P. J. Quinn, C. W. Stubbs, W. Sutherland, A. B. Tomaney, T. Vandehei, D. L. Welch, and The MACHO Collaboration. Macho project limits on black hole dark matter in the 1-30 m range. The Astrophysical Journal Letters, 550(2):L169, 2001. URL http://stacks.iop.org/1538-4357/550/ $i=2 / a=L 169$.

[19] S. D. M. White, M. Davis, and C. S. Frenk. The size of clusters in a neutrino-dominated universe. mnras, 209:27P-31P, July 1984. doi: 10.1093/mnras/209.1.27P.

[20] Steven Weinberg. A new light boson? Phys. Rev. Lett., 40:223-226, Jan 1978. doi: 10.1103/PhysRevLett.40.223. URL https://link. aps.org/doi/10.1103/PhysRevLett.40.223.

[21] F. Wilczek. Problem of strong $p$ and $t$ invariance in the presence of instantons. Phys. Rev. Lett., 40:279-282, Jan 1978. doi: 10.1103/PhysRevLett.40.279. URL https://link.aps.org/doi/10. 1103/PhysRevLett.40.279.

[22] Gerard Jungman, Marc Kamionkowski, and Kim Griest. Supersymmetric dark matter. Phys. Rept., 267:195-373, 1996. doi: 10.1016/ 0370-1573(95)00058-5.

[23] C. A. Baker, D. D. Doyle, P. Geltenbort, K. Green, M. G. D. van der Grinten, P. G. Harris, P. Iaydjiev, S. N. Ivanov, D. J. R. May, J. M. Pendlebury, J. D. Richardson, D. Shiers, and K. F. Smith. Improved experimental limit on the electric dipole moment of the neutron. Phys. Rev. Lett., 97:131801, Sep 2006. doi: 10.1103/PhysRevLett.97. 131801. URL https://link.aps.org/doi/10.1103/PhysRevLett. 97.131801.

[24] R. D. Peccei and Helen R. Quinn. Constraints imposed by CP conservation in the presence of pseudoparticles. Phys. Rev. D, 16: 1791-1797, Sep 1977. doi: 10.1103/PhysRevD.16.1791. URL https: //link.aps.org/doi/10.1103/PhysRevD.16.1791.

[25] Georg Raffelt. Stellar-evolution limits on axion properties. $\mathrm{Nu}$ clear Physics B - Proceedings Supplements, 72:43 - 53, 1999. 
ISSN 0920-5632. doi: https://doi.org/10.1016/S0920-5632(98) 00501-5. URL http://www.sciencedirect.com/science/article/ pii/S0920563298005015. Proceedings of the 5th IFT Workshop on Axions.

[26] N. Du, N. Force, R. Khatiwada, E. Lentz, R. Ottens, L. J Rosenberg, G. Rybka, G. Carosi, N. Woollett, D. Bowring, A. S. Chou, A. Sonnenschein, W. Wester, C. Boutan, N. S. Oblath, R. Bradley, E. J. Daw, A. V. Dixit, J. Clarke, S. R. O'Kelley, N. Crisosto, J. R. Gleason, S. Jois, P. Sikivie, I. Stern, N. S. Sullivan, D. B Tanner, and G. C. Hilton. Search for invisible axion dark matter with the axion dark matter experiment. Phys. Rev. Lett., 120: 151301, Apr 2018. doi: 10.1103/PhysRevLett.120.151301. URL https://link.aps.org/doi/10.1103/PhysRevLett.120.151301.

[27] Dan Hooper, Neal Weiner, and Wei Xue. Dark forces and light dark matter. Phys. Rev. D, 86:056009, Sep 2012. doi: 10.1103/PhysRevD. 86.056009. URL https://link.aps.org/doi/10.1103/PhysRevD. 86.056009 .

[28] R. Foot. Hidden sector dark matter explains the dama, cogent, cresstii and cdms/si experiments. Phys. Rev. D, 88:025032, Jul 2013. doi: 10.1103/PhysRevD.88.025032. URL https://link.aps.org/ doi/10.1103/PhysRevD.88.025032.

[29] Maxim Pospelov, Adam Ritz, and Mikhail Voloshin. Secluded wimp dark matter. Physics Letters B, 662(1):53 - 61, 2008. ISSN 0370-2693. doi: https://doi.org/10.1016/j.physletb.2008.02. 052. URL http://www.sciencedirect.com/science/article/pii/ S0370269308002402.

[30] Kalliopi Petraki and Raymond R. Volkas. Review of asymmetric dark matter. International Journal of Modern Physics A, 28(19):1330028, 2013. doi: 10.1142/S0217751X13300287. URL https://doi .org/10. 1142/S0217751X13300287.

[31] David B. Kaplan. Single explanation for both baryon and dark matter densities. Phys. Rev. Lett., 68:741-743, Feb 1992. doi: 10.1103/PhysRevLett.68.741. URL https://link.aps.org/doi/10. 1103/PhysRevLett.68.741.

[32] Kathryn M. Zurek. Asymmetric dark matter: Theories, signatures, and constraints. Physics Reports, 537(3):91 - 121, 2014. 
ISSN 0370-1573. doi: https://doi.org/10.1016/j.physrep.2013.12. 001. URL http://www.sciencedirect.com/science/article/pii/ S0370157313004341. Asymmetric Dark Matter: Theories, signatures, and constraints.

[33] Martin C. Smith, Gregory R. Ruchti, Amina Helmi, Rosemary F. G. Wyse, J. P. Fulbright, K. C. Freeman, J. F. Navarro, G. M. Seabroke, M. Steinmetz, M. Williams, O. Bienaym, J. Binney, J. BlandHawthorn, W. Dehnen, B. K. Gibson, G. Gilmore, E. K. Grebel, U. Munari, Q. A. Parker, R.-D. Scholz, A. Siebert, F. G. Watson, and T. Zwitter. The rave survey: constraining the local galactic escape speed. Monthly Notices of the Royal Astronomical Society, 379(2):755-772, 2007. doi: 10.1111/j.1365-2966.2007.11964.x. URL http://dx.doi.org/10.1111/j.1365-2966.2007.11964.x.

[34] J. Binney and S. Tremaine. Galactic Dynamics. Princeton University Press, 2 edition, 2008. ISBN 9781400828722.

[35] Miguel Pato, Oscar Agertz, Gianfranco Bertone, Ben Moore, and Romain Teyssier. Systematic uncertainties in the determination of the local dark matter density. Phys. Rev. D, 82:023531, Jul 2010. doi: 10.1103/PhysRevD.82.023531. URL https://link.aps.org/ doi/10.1103/PhysRevD.82.023531.

[36] J.D. Lewin and P.F. Smith. Review of mathematics, numerical factors, and corrections for dark matter experiments based on elastic nuclear recoil. Astroparticle Physics, 6(1):87 - 112, 1996. ISSN 0927-6505. doi: https://doi.org/10.1016/S0927-6505(96)00047-3. URL http://www . sciencedirect.com/science/article/pii/S0927650596000473.

[37] J I Read. The local dark matter density. Journal of Physics G: Nuclear and Particle Physics, 41(6):063101, 2014. URL http://stacks.iop. org $/ 0954-3899 / 41 / i=6 / a=063101$.

[38] Sebastian Baum, Katherine Freese, and Chris Kelso. Dark Matter implications of DAMA/LIBRA-phase2 results. 2018.

[39] Govinda Adhikari, Pushparaj Adhikari, Estella Barbosa de Souza, Nelson Carlin, Seonho Choi, Mitra Djamal, Anthony C. Ezeribe, Chang Hyon Ha, Insik Hahn, Antonia J. F. Hubbard, Eunju Jeon, Jay Hyun Jo, Hanwool Joo, Woon Gu Kang, Woosik Kang, Matthew Kauer, Bonghee Kim, Hongjoo Kim, Hyounggyu Kim, Kyungwon 
Kim, Nam Young Kim, Sun Kee Kim, Yeongduk Kim, Yong-Hamb Kim, Young Ju Ko, Vitaly A. Kudryavtsev, Hyun Su Lee, Jaison Lee, Jooyoung Lee, Moo Hyun Lee, Douglas S. Leonard, Warren A. Lynch, Reina H. Maruyama, Frederic Mouton, Stephen L. Olsen, Byungju Park, Hyang Kyu Park, Hyeonseo Park, Jungsic Park, Kangsoon Park, Walter C. Pettus, Hafizh Prihtiadi, Sejin Ra, Carsten Rott, Andrew Scarff, Keon Ah Shin, Neil J. C. Spooner, William G. Thompson, Liang Yang, Seok Hyun Yong, and The COSINE-100 Collaboration. An experiment to search for dark-matter interactions using sodium iodide detectors. Nature, 564(7734):83-86, 2018. doi: 10.1038/s41586-018-0739-1. URL https://doi.org/10.1038/ s41586-018-0739-1.

[40] F. Donato, N. Fornengo, and S. Scopel. Effects of galactic dark halo rotation on wimp direct detection. Astroparticle Physics, 9(3):247 - 260, 1998. ISSN 0927-6505. doi: https://doi.org/10.1016/S0927-6505(98) 00025-5. URL http://www.sciencedirect.com/science/article/ pii/S0927650598000255.

[41] Mark W. Goodman and Edward Witten. Detectability of Certain Dark Matter Candidates. Phys. Rev., D31:3059, 1985. doi: 10.1103/ PhysRevD.31.3059. [,325(1984)].

[42] S. Archambault et al. Constraints on Low-Mass WIMP Interactions on ${ }^{19} \mathrm{~F}$ from PICASSO. Phys. Lett., B711:153-161, 2012. doi: 10. 1016/j.physletb.2012.03.078.

[43] Andi Tan, Mengjiao Xiao, Xiangyi Cui, Xun Chen, Yunhua Chen, Deqing Fang, Changbo Fu, Karl Giboni, Franco Giuliani, Haowei Gong, Xuyuan Guo, Ke Han, Shouyang Hu, Xingtao Huang, Xiangdong Ji, Yonglin Ju, Siao Lei, Shaoli Li, Xiaomei Li, Xinglong Li, Hao Liang, Qing Lin, Huaxuan Liu, Jianglai Liu, Wolfgang Lorenzon, Yugang Ma, Yajun Mao, Kaixuan Ni, Xiangxiang Ren, Michael Schubnell, Manbin Shen, Fang Shi, Hongwei Wang, Jimin Wang, Meng Wang, Qiuhong Wang, Siguang Wang, Xuming Wang, Zhou Wang, Shiyong Wu, Xiang Xiao, Pengwei Xie, Binbin Yan, Yong Yang, Jianfeng Yue, Xionghui Zeng, Hongguang Zhang, Hua Zhang, Huanqiao Zhang, Tao Zhang, Li Zhao, Jing Zhou, Ning Zhou, and Xiaopeng Zhou. Dark Matter Results from First 98.7 Days of Data from the PandaX-II Experiment. Phys. Rev. Lett., 
117:121303, Sep 2016. doi: 10.1103/PhysRevLett.117.121303. URL https://link.aps.org/doi/10.1103/PhysRevLett.117.121303.

[44] E. Aprile, J. Aalbers, F. Agostini, M. Alfonsi, L. Althueser, F. D. Amaro, M. Anthony, F. Arneodo, L. Baudis, B. Bauermeister, M. L. Benabderrahmane, T. Berger, P. A. Breur, A. Brown, A. Brown, E. Brown, S. Bruenner, G. Bruno, R. Budnik, C. Capelli, J. M. R. Cardoso, D. Cichon, D. Coderre, A. P. Colijn, J. Conrad, J. P. Cussonneau, M. P. Decowski, P. de Perio, P. Di Gangi, A. Di Giovanni, S. Diglio, A. Elykov, G. Eurin, J. Fei, A. D. Ferella, A. Fieguth, W. Fulgione, A. Gallo Rosso, M. Galloway, F. Gao, M. Garbini, C. Geis, L. Grandi, Z. Greene, H. Qiu, C. Hasterok, E. Hogenbirk, J. Howlett, R. Itay, F. Joerg, B. Kaminsky, S. Kazama, A. Kish, G. Koltman, H. Landsman, R. F. Lang, L. Levinson, Q. Lin, S. Lindemann, M. Lindner, F. Lombardi, J. A. M. Lopes, J. Mahlstedt, A. Manfredini, T. Marrodán Undagoitia, J. Masbou, D. Masson, M. Messina, K. Micheneau, K. Miller, A. Molinario, K. Morå, M. Murra, J. Naganoma, K. Ni, U. Oberlack, B. Pelssers, F. Piastra, J. Pienaar, V. Pizzella, G. Plante, R. Podviianiuk, N. Priel, D. Ramírez García, L. Rauch, S. Reichard, C. Reuter, B. Riedel, A. Rizzo, A. Rocchetti, N. Rupp, J. M. F. dos Santos, G. Sartorelli, M. Scheibelhut, S. Schindler, J. Schreiner, D. Schulte, M. Schumann, L. Scotto Lavina, M. Selvi, P. Shagin, E. Shockley, M. Silva, H. Simgen, D. Thers, F. Toschi, G. Trinchero, C. Tunnell, N. Upole, M. Vargas, O. Wack, H. Wang, Z. Wang, Y. Wei, C. Weinheimer, C. Wittweg, J. Wulf, J. Ye, Y. Zhang, and T. Zhu. Dark matter search results from a one ton-year exposure of xenon1t. Phys. Rev. Lett., 121:111302, Sep 2018. doi: 10.1103/PhysRevLett.121.111302. URL https://link.aps.org/doi/10.1103/PhysRevLett.121.111302.

[45] D. S. Akerib, S. Alsum, H. M. Araújo, X. Bai, A. J. Bailey, J. Balajthy, P. Beltrame, E. P. Bernard, A. Bernstein, T. P. Biesiadzinski, E. M. Boulton, R. Bramante, P. Brás, D. Byram, S. B. Cahn, M. C. Carmona-Benitez, C. Chan, A. A. Chiller, C. Chiller, A. Currie, J. E. Cutter, T. J. R. Davison, A. Dobi, J. E. Y. Dobson, E. Druszkiewicz, B. N. Edwards, C. H. Faham, S. Fiorucci, R. J. Gaitskell, V. M. Gehman, C. Ghag, K. R. Gibson, M. G. D. Gilchriese, C. R. Hall, M. Hanhardt, S. J. Haselschwardt, S. A. Hertel, D. P. Hogan, M. Horn, D. Q. Huang, C. M. Ignarra, M. Ihm, R. G. Jacobsen, W. Ji, K. Kamdin, K. Kazkaz, D. Khaitan, R. Knoche, N. A. 
Larsen, C. Lee, B. G. Lenardo, K. T. Lesko, A. Lindote, M. I. Lopes, A. Manalaysay, R. L. Mannino, M. F. Marzioni, D. N. McKinsey, D.M. Mei, J. Mock, M. Moongweluwan, J. A. Morad, A. St. J. Murphy, C. Nehrkorn, H. N. Nelson, F. Neves, K. O'Sullivan, K. C. Oliver-Mallory, K. J. Palladino, E. K. Pease, P. Phelps, L. Reichhart, C. Rhyne, S. Shaw, T. A. Shutt, C. Silva, M. Solmaz, V. N. Solovov, P. Sorensen, S. Stephenson, T. J. Sumner, M. Szydagis, D. J. Taylor, W. C. Taylor, B. P. Tennyson, P. A. Terman, D. R. Tiedt, W. H. To, M. Tripathi, L. Tvrznikova, S. Uvarov, J. R. Verbus, R. C. Webb, J. T. White, T. J. Whitis, M. S. Witherell, F. L. H. Wolfs, J. Xu, K. Yazdani, S. K. Young, and C. Zhang. Results from a search for dark matter in the complete lux exposure. Phys. Rev. Lett., 118:021303, Jan 2017. doi: 10.1103/PhysRevLett.118.021303. URL https://link.aps.org/doi/10.1103/PhysRevLett.118.021303.

[46] D. Moore. A Search for Low-Mass Dark Matter with the Cryogenic Dark Matter Search and the Development of Highly Multiplexed Phonon-Mediated Particle Detectors. PhD thesis, California Institute of Technology, 2012. URL http://cdms.berkeley.edu/ Dissertations/moore.pdf.

[47] Vardan Khachatryan et al. Search for dark matter, extra dimensions, and unparticles in monojet events in protonproton collisions at $\sqrt{s}=8 \mathrm{TeV}$. Eur. Phys. J., C75(5):235, 2015. doi: 10.1140/epjc/ s10052-015-3451-4.

[48] Georges Aad et al. Search for new phenomena in final states with an energetic jet and large missing transverse momentum in pp collisions at $\sqrt{s}=8 \mathrm{TeV}$ with the ATLAS detector. Eur. Phys. J., C75 (7):299, 2015. doi: 10.1140/epjc/s10052-015-3517-3,10.1140/epjc/ s10052-015-3639-7. [Erratum: Eur. Phys. J.C75,no.9,408(2015)].

[49] A. A. Aguilar-Arevalo et al. Dark Matter Search in a Proton Beam Dump with MiniBooNE. Phys. Rev. Lett., 118(22):221803, 2017. doi: 10.1103/PhysRevLett.118.221803.

[50] Patrick deNiverville, Chien-Yi Chen, Maxim Pospelov, and Adam Ritz. Light dark matter in neutrino beams: production modelling and scattering signatures at MiniBooNE, T2K and SHiP. Phys. Rev., D95(3):035006, 2017. doi: 10.1103/PhysRevD.95.035006. 
[51] Filip Jediny, Athanasios Hatzikoutelis, Sergey Kotelnikov, and Biao Wang. Looking amongst the neutrinos for lightweight dark matter in the NOvA Near Detector. PoS, ICHEP2016:876, 2017. doi: 10.22323/ 1.282.0876.

[52] Eder Izaguirre, Yonatan Kahn, Gordan Krnjaic, and Matthew Moschella. Testing Light Dark Matter Coannihilation With FixedTarget Experiments. Phys. Rev., D96(5):055007, 2017. doi: 10.1103/ PhysRevD.96.055007.

[53] M. Battaglieri et al. The Heavy Photon Search Test Detector. Nucl. Instrum. Meth., A777:91-101, 2015. doi: 10.1016/j.nima.2014.12.017.

[54] W. B. Atwood, A. A. Abdo, M. Ackermann, W. Althouse, B. Anderson, M. Axelsson, L. Baldini, J. Ballet, D. L. Band, G. Barbiellini, J. Bartelt, D. Bastieri, B. M. Baughman, K. Bechtol, D. Bdrde, F. Bellardi, R. Bellazzini, B. Berenji, G. F. Bignami, D. Bisello, E. Bissaldi, R. D. Blandford, E. D. Bloom, J. R. Bogart, E. Bonamente, J. Bonnell, A. W. Borgland, A. Bouvier, J. Bregeon, A. Brez, M. Brigida, P. Bruel, T. H. Burnett, G. Busetto, G. A. Caliandro, R. A. Cameron, P. A. Caraveo, S. Carius, P. Carlson, J. M. Casandjian, E. Cavazzuti, M. Ceccanti, C. Cecchi, E. Charles, A. Chekhtman, C. C. Cheung, J. Chiang, R. Chipaux, A. N. Cillis, S. Ciprini, R. Claus, J. Cohen-Tanugi, S. Condamoor, J. Conrad, R. Corbet, L. Corucci, L. Costamante, S. Cutini, D. S. Davis, D. Decotigny, M. DeKlotz, C. D. Dermer, A. de Angelis, S. W. Digel, E. do Couto e Silva, P. S. Drell, R. Dubois, D. Dumora, Y. Edmonds, D. Fabiani, C. Farnier, C. Favuzzi, D. L. Flath, P. Fleury, W. B. Focke, S. Funk, P. Fusco, F. Gargano, D. Gasparrini, N. Gehrels, F.-X. Gentit, S. Germani, B. Giebels, N. Giglietto, P. Giommi, F. Giordano, T. Glanzman, G. Godfrey, I. A. Grenier, M.H. Grondin, J. E. Grove, L. Guillemot, S. Guiriec, G. Haller, A. K. Harding, P. A. Hart, E. Hays, S. E. Healey, M. Hirayama, L. Hjalmarsdotter, R. Horn, R. E. Hughes, G. Jhannesson, G. Johansson, A. S. Johnson, R. P. Johnson, T. J. Johnson, W. N. Johnson, T. Kamae, H. Katagiri, J. Kataoka, A. Kavelaars, N. Kawai, H. Kelly, M. Kerr, W. Klamra, J. Kndlseder, M. L. Kocian, N. Komin, F. Kuehn, M. Kuss, D. Landriu, L. Latronico, B. Lee, S.-H. Lee, M. LemoineGoumard, A. M. Lionetto, F. Longo, F. Loparco, B. Lott, M. N. Lovellette, P. Lubrano, G. M. Madejski, A. Makeev, B. Marangelli, M. M. Massai, M. N. Mazziotta, J. E. McEnery, N. Menon, C. Meurer, P. F. Michelson, M. Minuti, N. Mirizzi, W. Mitthumsiri, T. Mizuno, 
A. A. Moiseev, C. Monte, M. E. Monzani, E. Moretti, A. Morselli, I. V. Moskalenko, S. Murgia, T. Nakamori, S. Nishino, P. L. Nolan, J. P. Norris, E. Nuss, M. Ohno, T. Ohsugi, N. Omodei, E. Orlando, J. F. Ormes, A. Paccagnella, D. Paneque, J. H. Panetta, D. Parent, M. Pearce, M. Pepe, A. Perazzo, M. Pesce-Rollins, P. Picozza, L. Pieri, M. Pinchera, F. Piron, T. A. Porter, L. Poupard, S. Rain, R. Rando, E. Rapposelli, M. Razzano, A. Reimer, O. Reimer, T. Reposeur, L. C. Reyes, S. Ritz, L. S. Rochester, A. Y. Rodriguez, R. W. Romani, M. Roth, J. J. Russell, F. Ryde, S. Sabatini, H. F.-W. Sadrozinski, D. Sanchez, A. Sander, L. Sapozhnikov, P. M. Saz Parkinson, J. D. Scargle, T. L. Schalk, G. Scolieri, C. Sgr, G. H. Share, M. Shaw, T. Shimokawabe, C. Shrader, A. Sierpowska-Bartosik, E. J. Siskind, D. A. Smith, P. D. Smith, G. Spandre, P. Spinelli, J.-L. Starck, T. E. Stephens, M. S. Strickman, A. W. Strong, D. J. Suson, H. Tajima, H. Takahashi, T. Takahashi, T. Tanaka, A. Tenze, S. Tether, J. B. Thayer, J. G. Thayer, D. J. Thompson, L. Tibaldo, O. Tibolla, D. F. Torres, G. Tosti, A. Tramacere, M. Turri, T. L. Usher, N. Vilchez, V. Vitale, P. Wang, K. Watters, B. L. Winer, K. S. Wood, T. Ylinen, and M. Ziegler. The large area telescope on the fermi gamma-ray space telescope mission. The Astrophysical Journal, 697(2):1071, 2009. URL http://stacks.iop.org/0004-637X/697/i=2/a=1071.

[55] Tansu Daylan, Douglas P. Finkbeiner, Dan Hooper, Tim Linden, Stephen K.N. Portillo, Nicholas L. Rodd, and Tracy R. Slatyer. The characterization of the gamma-ray signal from the central milky way: A case for annihilating dark matter. Physics of the Dark Universe, 12:1 - 23, 2016. ISSN 2212-6864. doi: https://doi.org/10.1016/j. dark.2015.12.005. URL http://www.sciencedirect.com/science/ article/pii/S2212686416000030.

[56] Samuel K. Lee, Mariangela Lisanti, Benjamin R. Safdi, Tracy R. Slatyer, and Wei Xue. Evidence for unresolved $\gamma$-ray point sources in the inner galaxy. Phys. Rev. Lett., 116:051103, Feb 2016. doi: 10. 1103/PhysRevLett.116.051103. URL https://link.aps.org/doi/ 10.1103/PhysRevLett.116.051103.

[57] Richard Bartels and Christoph Weniger. Millisecond Pulsars in the Galactic Bulge? An Extended Discussion on the Wavelet Analysis of the Fermi-LAT data. IAU Symp., 322:193-196, 2016. doi: 10.1017/ S174392131601200X. 
[58] M. G. Aartsen, R. Abbasi, Y. Abdou, M. Ackermann, J. Adams, J. A. Aguilar, M. Ahlers, D. Altmann, J. Auffenberg, X. Bai, M. Baker, S. W. Barwick, V. Baum, R. Bay, K. Beattie, J. J. Beatty, S. Bechet, J. Becker Tjus, K.-H. Becker, M. Bell, M. L. Benabderrahmane, S. BenZvi, J. Berdermann, P. Berghaus, D. Berley, E. Bernardini, A. Bernhard, D. Bertrand, D. Z. Besson, D. Bindig, M. Bissok, E. Blaufuss, J. Blumenthal, D. J. Boersma, S. Bohaichuk, C. Bohm, D. Bose, S. Böser, O. Botner, L. Brayeur, A. M. Brown, R. Bruijn, J. Brunner, S. Buitink, M. Carson, J. Casey, M. Casier, D. Chirkin, B. Christy, K. Clark, F. Clevermann, S. Cohen, D. F. Cowen, A. H. Cruz Silva, M. Danninger, J. Daughhetee, J. C. Davis, C. De Clercq, S. De Ridder, P. Desiati, G. de Vries-Uiterweerd, M. de With, T. DeYoung, J. C. Díaz-Vélez, J. Dreyer, M. Dunkman, R. Eagan, B. Eberhardt, J. Eisch, R. W. Ellsworth, O. Engdegård, S. Euler, P. A. Evenson, O. Fadiran, A. R. Fazely, A. Fedynitch, J. Feintzeig, T. Feusels, K. Filimonov, C. Finley, T. Fischer-Wasels, S. Flis, A. Franckowiak, R. Franke, K. Frantzen, T. Fuchs, T. K. Gaisser, J. Gallagher, L. Gerhardt, L. Gladstone, T. Glüsenkamp, A. Goldschmidt, G. Golup, J. A. Goodman, D. Góra, D. Grant, A. Groß, M. Gurtner, C. Ha, A. Haj Ismail, A. Hallgren, F. Halzen, K. Hanson, D. Heereman, P. Heimann, D. Heinen, K. Helbing, R. Hellauer, S. Hickford, G. C. Hill, K. D. Hoffman, R. Hoffmann, A. Homeier, K. Hoshina, W. Huelsnitz, P. O. Hulth, K. Hultqvist, S. Hussain, A. Ishihara, E. Jacobi, J. Jacobsen, G. S. Japaridze, K. Jero, O. Jlelati, B. Kaminsky, A. Kappes, T. Karg, A. Karle, J. L. Kelley, J. Kiryluk, F. Kislat, J. Kläs, S. R. Klein, J.-H. Köhne, G. Kohnen, H. Kolanoski, L. Köpke, C. Kopper, S. Kopper, D. J. Koskinen, M. Kowalski, M. Krasberg, G. Kroll, J. Kunnen, N. Kurahashi, T. Kuwabara, M. Labare, H. Landsman, M. J. Larson, M. Lesiak-Bzdak, J. Leute, J. Lünemann, J. Madsen, R. Maruyama, K. Mase, H. S. Matis, F. McNally, K. Meagher, M. Merck, P. Mészáros, T. Meures, S. Miarecki, E. Middell, N. Milke, J. Miller, L. Mohrmann, T. Montaruli, R. Morse, R. Nahnhauer, U. Naumann, H. Niederhausen, S. C. Nowicki, D. R. Nygren, A. Obertacke, S. Odrowski, A. Olivas, M. Olivo, A. O'Murchadha, L. Paul, J. A. Pepper, C. Pérez de los Heros, C. Pfendner, D. Pieloth, N. Pirk, J. Posselt, P. B. Price, G. T. Przybylski, L. Rädel, K. Rawlins, P. Redl, E. Resconi, W. Rhode, M. Ribordy, M. Richman, B. Riedel, J. P. Rodrigues, C. Rott, T. Ruhe, B. Ruzybayev, D. Ryckbosch, S. M. Saba, T. Salameh, H.-G. Sander, M. Santander, S. Sarkar, K. Schatto, M. Scheel, F. Scheriau, T. Schmidt, M. Schmitz, S. Schoe- 
nen, S. Schöneberg, L. Schönherr, A. Schönwald, A. Schukraft, L. Schulte, O. Schulz, D. Seckel, S. H. Seo, Y. Sestayo, S. Seunarine, C. Sheremata, M. W. E. Smith, M. Soiron, D. Soldin, G. M. Spiczak, C. Spiering, M. Stamatikos, T. Stanev, A. Stasik, T. Stezelberger, R. G. Stokstad, A. Stößl, E. A. Strahler, R. Ström, G. W. Sullivan, H. Taavola, I. Taboada, A. Tamburro, S. Ter-Antonyan, S. Tilav, P. A. Toale, S. Toscano, M. Usner, D. van der Drift, N. van Eijndhoven, A. Van Overloop, J. van Santen, M. Vehring, M. Voge, M. Vraeghe, C. Walck, T. Waldenmaier, M. Wallraff, R. Wasserman, Ch. Weaver, M. Wellons, C. Wendt, S. Westerhoff, N. Whitehorn, K. Wiebe, C. H. Wiebusch, D. R. Williams, H. Wissing, M. Wolf, T. R. Wood, K. Woschnagg, C. Xu, D. L. Xu, X. W. Xu, J. P. Yanez, G. Yodh, S. Yoshida, P. Zarzhitsky, J. Ziemann, S. Zierke, A. Zilles, and M. Zoll. Search for dark matter annihilations in the sun with the 79-string icecube detector. Phys. Rev. Lett., 110: 131302, Mar 2013. doi: 10.1103/PhysRevLett.110.131302. URL https://link.aps.org/doi/10.1103/PhysRevLett.110.131302.

[59] K. Choi, K. Abe, Y. Haga, Y. Hayato, K. Iyogi, J. Kameda, Y. Kishimoto, M. Miura, S. Moriyama, M. Nakahata, Y. Nakano, S. Nakayama, H. Sekiya, M. Shiozawa, Y. Suzuki, A. Takeda, T. Tomura, R. A. Wendell, T. Irvine, T. Kajita, I. Kametani, K. Kaneyuki, K. P. Lee, Y. Nishimura, K. Okumura, T. McLachlan, L. Labarga, E. Kearns, J. L. Raaf, J. L. Stone, L. R. Sulak, S. Berkman, H. A. Tanaka, S. Tobayama, M. Goldhaber, G. Carminati, W. R. Kropp, S. Mine, A. Renshaw, M. B. Smy, H. W. Sobel, K. S. Ganezer, J. Hill, N. Hong, J. Y. Kim, I. T. Lim, T. Akiri, A. Himmel, K. Scholberg, C. W. Walter, T. Wongjirad, T. Ishizuka, S. Tasaka, J. S. Jang, J. G. Learned, S. Matsuno, S. N. Smith, T. Hasegawa, T. Ishida, T. Ishii, T. Kobayashi, T. Nakadaira, K. Nakamura, Y. Oyama, K. Sakashita, T. Sekiguchi, T. Tsukamoto, A. T. Suzuki, Y. Takeuchi, C. Bronner, S. Hirota, K. Huang, K. Ieki, M. Ikeda, T. Kikawa, A. Minamino, T. Nakaya, K. Suzuki, S. Takahashi, Y. Fukuda, Y. Itow, G. Mitsuka, P. Mijakowski, J. Hignight, J. Imber, C. K. Jung, C. Yanagisawa, H. Ishino, A. Kibayashi, Y. Koshio, T. Mori, M. Sakuda, T. Yano, Y. Kuno, R. Tacik, S. B. Kim, H. Okazawa, Y. Choi, K. Nishijima, M. Koshiba, Y. Totsuka, M. Yokoyama, K. Martens, Ll. Marti, M. R. Vagins, J. F. Martin, P. de Perio, A. Konaka, M. J. Wilking, S. Chen, Y. Zhang, and R. J. Wilkes. Search for neutrinos from annihilation of captured low-mass dark matter parti- 
cles in the sun by super-kamiokande. Phys. Rev. Lett., 114:141301, Apr 2015. doi: 10.1103/PhysRevLett.114.141301. URL https: //link.aps.org/doi/10.1103/PhysRevLett.114.141301.

[60] Mariangela Lisanti. Lectures on Dark Matter Physics. In Proceedings, Theoretical Advanced Study Institute in Elementary Particle Physics: New Frontiers in Fields and Strings (TASI 2015): Boulder, CO, USA, June 1-26, 2015, pages 399-446, 2017. doi: 10.1142/9789813149441_ 0007.

[61] G. Bertolini, A. Coche. Semiconductor Detectors. North-Holland Publishing Company, 1968.

[62] R. C. Alig and S. Bloom. Electron-hole-pair creation energies in semiconductors. Phys. Rev. Lett., 35:1522-1525, Dec 1975. doi: 10.1103/PhysRevLett.35.1522. URL https://link.aps.org/doi/ 10.1103/PhysRevLett.35.1522.

[63] Jens Lindhard, Morten Scharff, and H. E. Schiott. Range Concepts and Heavy Ion Ranges (Notes on Atomic Collisions, II). Mat. Fys. Medd. K. Dan. Vid. Selsk., 33(14), 1963.

[64] Jens Lindhard, Vibeke Nielsen, and Morten Scharff. Approximation Method in Classical Scattering by Screened Coulomb Fields (Notes on Atomic Collisions, I). Mat. Fys. Medd. K. Dan. Vid. Selsk., 36(10), 1968.

[65] Jens Lindhard, Vibeke Nielsen, Morten Scharff, and P. V. Thomsen. Integral Equations Governing Radiation Effects (Notes on Atomic Collisions, III). Mat. Fys. Medd. K. Dan. Vid. Selsk., 33(10), 1963.

[66] S. Hertel. Advancing the Search for Dark Matter: from CDMS II to SuperCDMS. PhD thesis, Massachusetts Institute of Technology, 2012. URL http://cdms.berkeley.edu/Dissertations/hertel.pdf.

[67] Mark David Pepin. Low-Mass Dark Matter Search Results and Radiogenic Backgrounds for the Cryogenic Dark Matter Search. PhD thesis, University of Minnesota, 2016. URL http://cdms.berkeley. edu/Dissertations/pepin.pdf.

[68] K. W. Jones and H. W. Kraner. Stopping of 1- to 1.8-keV ${ }^{73}$ Ge Atoms in Germanium. Phys. Rev. C, 4(1):125-129, jul 1971. ISSN 05562813. doi: 10.1103/PhysRevC.4.125. URL https://link.aps.org/ doi/10.1103/PhysRevC.4.125. 
[69] K. W. Jones and H. W. Kraner. Energy lost to ionization by 254eV ${ }^{73}$ Ge atoms stopping in Ge. Phys. Rev. A, 11(4):1347-1353, apr 1975. ISSN 0556-2791. doi: 10.1103/PhysRevA.11.1347. URL https: //link.aps.org/doi/10.1103/PhysRevA.11.1347.

[70] A. R. Sattler, F. L. Vook, and J. M. Palms. Ionization Produced by Energetic Germanium Atoms within a Germanium Lattice. Phys. Rev., 143(2):588-594, mar 1966. ISSN 0031-899X. doi: 10.1103/PhysRev. 143.588. URL https://link.aps.org/doi/10.1103/PhysRev.143. 588.

[71] Y. Messous, B. Chambon, V. Chazal, M. De Jésus, D. Drain, C. Pastor, A. de Bellefon, M. Chapellier, G. Chardin, E. Gaillard-Lecanu, G. Gerbier, Y. Giraud-Héraud, D. Lhote, J. Mallet, L. Mosca, M.C. Perillo-Isaac, C. Tao, and D. Yvon. Calibration of a Ge crystal with nuclear recoils for the development of a dark matter detector. Astropart. Phys., 3(4):361-366, aug 1995. ISSN 0927-6505. doi: 10.1016/0927-6505(95)00007-4. URL https ://www. sciencedirect. com/science/article/pii/0927650595000074.

[72] P S Barbeau, J I Collar, and O Tench. Large-mass ultralow noise germanium detectors: performance and applications in neutrino and astroparticle physics. JCAP, 2007(09):009-009, sep 2007. ISSN 1475-7516. doi: 10.1088/1475-7516/2007/09/ 009. URL http://stacks.iop.org/1475-7516/2007/i=09/a=009? key=crossref . 81960450665b4416e0ec40a61866127f.

[73] D. Barker and D.-M. Mei. Germanium detector response to nuclear recoils in searching for dark matter. Astropart. Phys., 38:1-6, oct 2012. ISSN 0927-6505. doi: 10.1016/J.ASTROPARTPHYS.2012. 08.006. URL https://www.sciencedirect.com/science/article/ pii/S0927650512001521.

[74] Kevin McCarthy. http://cdms.berkeley.edu/Dissertations/mccarthy.pdf. PhD thesis, Massachusetts Institute of Technology, 2013. URL http://cdms. berkeley.edu/Dissertations/mccarthy.pdf.

[75] B.S. Neganov and V.N. Trofimov. Calorimetric method measuring ionizing radiation. Otkrytia $i$ Izobret., 146:215, 1985. URL http: //inspirehep.net/record/1416918.

[76] P. N. Luke. Voltage-assisted calorimetric ionization detector. J. Appl. Phys., 64(12):6858, 1988. ISSN 00218979. doi: 10.1063/1. 
341976. URL http://scitation.aip.org/content/aip/journal/ jap/64/12/10.1063/1.341976.

[77] J.P. Filippini. A Search for WIMP Dark Matter Using the First FiveTower Run of the Cryogenic Dark Matter Search. PhD thesis, University of California, Berkeley, 2008. URL http://cdms. berkeley.edu/ Dissertations/filippini.pdf.

[78] K.D. Irwin and G.C. Hilton. Transition-Edge Sensors, pages 63-150. Springer Berlin Heidelberg, Berlin, Heidelberg, 2005. ISBN 978-3-54031478-3. doi: 10.1007/10933596_3. URL https://doi.org/10.1007/ 10933596_3.

[79] M. Pyle. Non-stationary Optimum Filter: the dream of no position correction. CDMS Intern. iZIP R\&D Ebook, note 17.5, 2010. URL http://titus.stanford.edu/cdms_restricted/ mpyle1/ebooks/IZIP2/YPt_poscor_free/Ypt_pcfree.html.

[80] A. Villano. Non-Stationary OF (for cdmsbats) Usage Primer. CDMS Intern. Run 133-135 Anal. Ebook, HT1, 2013. URL http://titus.stanford.edu/cdms_restricted/Soudan/R133/ ebook/130113/index.html.

[81] R. Agnese, A. J. Anderson, D. Balakishiyeva, R. Basu Thakur, D. A. Bauer, A. Borgland, D. Brandt, P. L. Brink, R. Bunker, B. Cabrera, D. O. Caldwell, D. G. Cerdeno, H. Chagani, M. Cherry, J. Cooley, B. Cornell, C. H. Crewdson, P. Cushman, M. Daal, P. C. F. Di Stefano, E. Do Couto E Silva, T. Doughty, L. Esteban, S. Fallows, E. Figueroa-Feliciano, J. Fox, M. Fritts, G. L. Godfrey, S. R. Golwala, J. Hall, H. R. Harris, J. Hasi, S. A. Hertel, B. A. Hines, T. Hofer, D. Holmgren, L. Hsu, M. E. Huber, A. Jastram, O. Kamaev, B. Kara, M. H. Kelsey, S. A. Kenany, A. Kennedy, C. J. Kenney, M. Kiveni, K. Koch, B. Loer, E. Lopez Asamar, R. Mahapatra, V. Mandic, C. Martinez, K. A. McCarthy, N. Mirabolfathi, R. A. Moffatt, D. C. Moore, P. Nadeau, R. H. Nelson, L. Novak, K. Page, R. Partridge, M. Pepin, A. Phipps, K. Prasad, M. Pyle, H. Qiu, R. Radpour, W. Rau, P. Redl, A. Reisetter, R. W. Resch, Y. Ricci, T. Saab, B. Sadoulet, J. Sander, R. Schmitt, K. Schneck, R. W. Schnee, S. Scorza, D. Seitz, B. Serfass, B. Shank, D. Speller, A. Tomada, A. N. Villano, B. Welliver, D. H. Wright, S. Yellin, J. J. Yen, B. A. Young, and J. Zhang. Demonstration of surface electron rejection with interleaved germanium detectors for dark mat- 
ter searches. Applied Physics Letters, 103(16):164105, 2013. doi: 10.1063/1.4826093. URL https://doi.org/10.1063/1.4826093.

[82] R. Agnese, A. J. Anderson, T. Aralis, T. Aramaki, I. J. Arnquist, W. Baker, D. Balakishiyeva, D. Barker, R. Basu Thakur, D. A. Bauer, T. Binder, M. A. Bowles, P. L. Brink, R. Bunker, B. Cabrera, D. O. Caldwell, R. Calkins, C. Cartaro, D. G. Cerdeño, Y. Chang, H. Chagani, Y. Chen, J. Cooley, B. Cornell, P. Cushman, M. Daal, P. C. F. Di Stefano, T. Doughty, L. Esteban, E. Fascione, E. Figueroa-Feliciano, M. Fritts, G. Gerbier, M. Ghaith, G. L. Godfrey, S. R. Golwala, J. Hall, H. R. Harris, Z. Hong, E. W. Hoppe, L. Hsu, M. E. Huber, V. Iyer, D. Jardin, A. Jastram, C. Jena, M. H. Kelsey, A. Kennedy, A. Kubik, N. A. Kurinsky, A. Leder, B. Loer, E. Lopez Asamar, P. Lukens, D. MacDonell, R. Mahapatra, V. Mandic, N. Mast, E. H. Miller, N. Mirabolfathi, R. A. Moffatt, B. Mohanty, J. D. Morales Mendoza, J. Nelson, J. L. Orrell, S. M. Oser, K. Page, W. A. Page, R. Partridge, M. Pepin, M. Peñalver Martinez, A. Phipps, S. Poudel, M. Pyle, H. Qiu, W. Rau, P. Redl, A. Reisetter, T. Reynolds, A. Roberts, A. E. Robinson, H. E. Rogers, T. Saab, B. Sadoulet, J. Sander, K. Schneck, R. W. Schnee, S. Scorza, K. Senapati, B. Serfass, D. Speller, M. Stein, J. Street, H. A. Tanaka, D. Toback, R. Underwood, A. N. Villano, B. von Krosigk, B. Welliver, J. S. Wilson, M. J. Wilson, D. H. Wright, S. Yellin, J. J. Yen, B. A. Young, X. Zhang, and X. Zhao. Lowmass dark matter search with CDMSlite. Phys. Rev. D, 97(2):022002, jan 2018. ISSN 2470-0010. doi: 10.1103/PhysRevD.97.022002. URL https://link.aps.org/doi/10.1103/PhysRevD.97.022002.

[83] R.B. Thakur. The Cryogenic Dark Matter Search Low IonizationThreshold Experiment. $\mathrm{PhD}$ thesis, University of Illinois at Urbana-Champaign, 2015. URL http://cdms.berkeley.edu/ Dissertations/basuthakur.pdf.

[84] P. Agnes, I. F. M. Albuquerque, T. Alexander, A. K. Alton, G. R. Araujo, D. M. Asner, M. P. Ave, H. O. Back, B. Baldin, G. Batignani, K. Biery, V. Bocci, G. Bonfini, W. Bonivento, B. Bottino, F. Budano, S. Bussino, M. Cadeddu, M. Cadoni, F. Calaprice, A. Caminata, N. Canci, A. Candela, M. Caravati, M. Cariello, M. Carlini, M. Carpinelli, S. Catalanotti, V. Cataudella, P. Cavalcante, S. Cavuoti, A. Chepurnov, C. Cicalò, L. Cifarelli, A. G. Cocco, G. Covone, D. D'Angelo, M. D'Incecco, D. D'Urso, S. Davini, A. De Candia, S. De Cecco, M. De Deo, G. De Filippis, G. De Rosa, M. De Vin- 
cenzi, P. Demontis, A. V. Derbin, A. Devoto, F. Di Eusanio, G. Di Pietro, C. Dionisi, M. Downing, E. Edkins, A. Empl, A. Fan, G. Fiorillo, K. Fomenko, D. Franco, F. Gabriele, A. Gabrieli, C. Galbiati, P. Garcia Abia, S. Giagu, C. Giganti, G. K. Giovanetti, O. Gorchakov, A. M. Goretti, F. Granato, M. Gromov, M. Guan, Y. Guardincerri, M. Gulino, B. R. Hackett, M. H. Hassanshahi, K. Herner, B. Hosseini, D. Hughes, P. Humble, E. V. Hungerford, Al. Ianni, An. Ianni, V. Ippolito, I. James, T. N. Johnson, Y. Kahn, K. Keeter, C. L. Kendziora, I. Kochanek, G. Koh, D. Korablev, G. Korga, A. Kubankin, M. Kuss, M. La Commara, M. Lai, X. Li, M. Lisanti, M. Lissia, B. Loer, G. Longo, Y. Ma, A. A. Machado, I. N. Machulin, A. Mandarano, L. Mapelli, S. M. Mari, J. Maricic, C. J. Martoff, A. Messina, P. D. Meyers, R. Milincic, S. Mishra-Sharma, A. Monte, M. Morrocchi, B. J. Mount, V. N. Muratova, P. Musico, R. Nania, A. Navrer Agasson, A. O. Nozdrina, A. Oleinik, M. Orsini, F. Ortica, L. Pagani, M. Pallavicini, L. Pandola, E. Pantic, E. Paoloni, F. Pazzona, K. Pelczar, N. Pelliccia, V. Pesudo, A. Pocar, S. Pordes, S. S. Poudel, D. A. Pugachev, H. Qian, F. Ragusa, M. Razeti, A. Razeto, B. Reinhold, A. L. Renshaw, M. Rescigno, Q. Riffard, A. Romani, B. Rossi, N. Rossi, D. Sablone, O. Samoylov, W. Sands, S. Sanfilippo, M. Sant, R. Santorelli, C. Savarese, E. Scapparone, B. Schlitzer, E. Segreto, D. A. Semenov, A. Shchagin, A. Sheshukov, P. N. Singh, M. D. Skorokhvatov, O. Smirnov, A. Sotnikov, C. Stanford, S. Stracka, G. B. Suffritti, Y. Suvorov, R. Tartaglia, G. Testera, A. Tonazzo, P. Trinchese, E. V. Unzhakov, M. Verducci, A. Vishneva, B. Vogelaar, M. Wada, T. J. Waldrop, H. Wang, Y. Wang, A. W. Watson, S. Westerdale, M. M. Wojcik, M. Wojcik, X. Xiang, X. Xiao, C. Yang, Z. Ye, C. Zhu, A. Zichichi, and G. Zuzel. Low-mass Dark Matter Search with the DarkSide-50 Experiment. feb 2018. URL http://arxiv.org/abs/1802.06994.

[85] W. Hampel and L. P. Remsberg. Half-life of ${ }^{71}$ Ge. Phys. Rev. C, 31(2): 666-667, feb 1985. ISSN 0556-2813. doi: 10.1103/PhysRevC.31.666. URL https://link.aps.org/doi/10.1103/PhysRevC.31.666.

[86] J. A. Bearden and A. F. Burr. Reevaluation of X-Ray Atomic Energy Levels. Rev. Mod. Phys., 39(1):125-142, jan 1967. ISSN 00346861. doi: 10.1103/RevModPhys.39.125. URL https://link.aps. org/doi/10.1103/RevModPhys.39.125.

[87] E. Schnfeld. Calculation of fractional electron capture prob- 
abilities. Applied Radiation and Isotopes, 49(9):1353 - 1357, 1998. ISSN 0969-8043. doi: https://doi.org/10.1016/S0969-8043(97) 10073-2. URL http://www.sciencedirect.com/science/article/ pii/S0969804397100732.

[88] J. Hall. R133 detector summary. CDMS Intern. Run 133-135 Anal. Ebook, HT167, 2013. URL http://titus.stanford.edu/cdms_ restricted/Soudan/R133/ebook/130422/ElectronCaptureProb . html.

[89] K. M. Sundqvist. Carrier Transport and Related Effects in Detectors of the Cryogenic Dark Matter Search. PhD thesis, University of California, Berkeley, 2012. URL http://cdms.berkeley.edu/ Dissertations/sundqvist.pdf.

[90] J. Morales M. Pyle. Correlation and Phase of Electronics Noise. CDMS Intern. Run 133-135 Anal. Ebook, HT269, 2014. URL http://titus. stanford.edu/cdms_restricted/Soudan/R133/ebook/140910_jm/.

[91] W. Rau. New Position Estimators based on 2-template quantities. CDMS Intern. Run 133-135 Anal. Ebook, 60lite2, 2015. URL http://titus.stanford.edu/cdms_restricted/Soudan/R133/ ebook/150707_wr/.

[92] S. Yellin. Finding an upper limit in the presence of an unknown background. Phys. Rev. D, 66(3):032005, aug 2002. ISSN 05562821. doi: 10.1103/PhysRevD.66.032005. URL http://journals. aps.org/prd/abstract/10.1103/PhysRevD.66.032005.

[93] S. Yellin. Extending the optimum interval method. page 12, sep 2007. URL http://arxiv.org/abs/0709.2701.

[94] G. Angloher, A. Bento, C. Bucci, L. Canonica, X. Defay, A. Erb, F. von Feilitzsch, N. Ferreiro Iachellini, P. Gorla, A. Gütlein, D. Hauff, J. Jochum, M. Kiefer, H. Kluck, H. Kraus, J. C. Lanfranchi, J. Loebell, A. Münster, C. Pagliarone, F. Petricca, W. Potzel, F. Pröbst, F. Reindl, K. Schäffner, J. Schieck, S. Schönert, W. Seidel, L. Stodolsky, C. Strandhagen, R. Strauss, A. Tanzke, H. H. Trinh Thi, C. Türkoğlu, M. Uffinger, A. Ulrich, I. Usherov, S. Wawoczny, M. Willers, M. Wüstrich, and A. Zöller. Results on light dark matter particles with a low-threshold CRESST-II detector. Eur. Phys. J. C, 76(1):25, Jan 2016. ISSN 1434-6052. doi: 
10.1140/epjc/s10052-016-3877-3. URL https://doi.org/10.1140/ epjc/s10052-016-3877-3.

[95] M. Pyle. Optimizing the Design and Analysis of Cryogenic Semiconductor Dark Matter Detectors for Maximum Sensitivity. PhD thesis, Stanford University, 2002. URL http://cdms.berkeley.edu/ Dissertations/pyle.pdf.

[96] B. Welliver. R133 Charge OF Noise Donuts From Low Bg using Optimal Filter Quantities. CDMS Intern. Run 133-135 Anal. Ebook, 2012. URL http://titus.stanford.edu/cdms_restricted/ Soudan/R133/ebook/120423_q/120423_q.html.

[97] R. Agnese, A. J. Anderson, T. Aramaki, I. Arnquist, W. Baker, D. Barker, R. Basu Thakur, D. A. Bauer, A. Borgland, M. A. Bowles, P. L. Brink, R. Bunker, B. Cabrera, D. O. Caldwell, R. Calkins, C. Cartaro, D. G. Cerdeño, H. Chagani, Y. Chen, J. Cooley, B. Cornell, P. Cushman, M. Daal, P. C. F. Di Stefano, T. Doughty, L. Esteban, S. Fallows, E. Figueroa-Feliciano, M. Fritts, G. Gerbier, M. Ghaith, G. L. Godfrey, S. R. Golwala, J. Hall, H. R. Harris, T. Hofer, D. Holmgren, Z. Hong, E. Hoppe, L. Hsu, M. E. Huber, V. Iyer, D. Jardin, A. Jastram, M. H. Kelsey, A. Kennedy, A. Kubik, N. A. Kurinsky, A. Leder, B. Loer, E. Lopez Asamar, P. Lukens, R. Mahapatra, V. Mandic, N. Mast, N. Mirabolfathi, R. A. Moffatt, J. D. Morales Mendoza, J. L. Orrell, S. M. Oser, K. Page, W. A. Page, R. Partridge, M. Pepin, A. Phipps, S. Poudel, M. Pyle, H. Qiu, W. Rau, P. Redl, A. Reisetter, A. Roberts, A. E. Robinson, H. E. Rogers, T. Saab, B. Sadoulet, J. Sander, K. Schneck, R. W. Schnee, B. Serfass, D. Speller, M. Stein, J. Street, H. A. Tanaka, D. Toback, R. Underwood, A. N. Villano, B. von Krosigk, B. Welliver, J. S. Wilson, D. H. Wright, S. Yellin, J. J. Yen, B. A. Young, X. Zhang, and X. Zhao. Projected sensitivity of the SuperCDMS SNOLAB experiment. Phys. Rev. D, 95(8):082002, apr 2017. ISSN 2470-0010. doi: 10.1103/PhysRevD.95.082002. URL http://link.aps.org/doi/10.1103/PhysRevD.95.082002.

[98] R. K. Romani, P. L. Brink, B. Cabrera, M. Cherry, T. Howarth, N. Kurinsky, R. A. Moffatt, R. Partridge, F. Ponce, M. Pyle, A. Tomada, S. Yellin, J. J. Yen, and B. A. Young. Thermal detection of single e-h pairs in a biased silicon crystal detector. Applied 
Physics Letters, 112(4):043501, 2018. doi: 10.1063/1.5010699. URL https://doi.org/10.1063/1.5010699.

[99] W. Schottky. ber spontane stromschwankungen in verschiedenen elektrizittsleitern. Annalen der Physik, 362(23):541-567. doi: 10.1002/ andp.19183622304. URL https://onlinelibrary.wiley.com/doi/ abs/10.1002/andp.19183622304.

[100] Frank Rice. A frequency-domain derivation of shot-noise. American Journal of Physics, 84(1):44-51, 2016. doi: 10.1119/1.4934706. URL https://doi.org/10.1119/1.4934706.

[101] M. J. Persky. Review of black surfaces for space-borne infrared systems. Review of Scientific Instruments, 70(5):2193-2217, 1999. doi: 10.1063/1.1149739. URL https://doi.org/10.1063/1.114973,.

[102] M. Pepin and R. Basu Thakur. CDMSlite Run 2 Cryo Cooler Noise/ Noise Monitor. CDMS Intern. Run 133-135 Anal. Ebook, 18lite2, 2014. URL http://titus.stanford.edu/cdms_restricted/ Soudan/R133/ebook/140616/note2.html.

[103] David Arthur and Sergei Vassilvitskii. K-means++: The advantages of careful seeding. In Proceedings of the Eighteenth Annual ACMSIAM Symposium on Discrete Algorithms, SODA '07, pages 10271035, Philadelphia, PA, USA, 2007. Society for Industrial and Applied Mathematics. ISBN 978-0-898716-24-5. URL http://dl.acm.org/ citation. cfm?id=1283383.1283494.

[104] Reuben Walter Ogburn. A Seach For Particle Dark Matter Using Cryogenic Germanium And Silicon Detectors In The One And TwoTower Runs Of CDMS-II At Soudan. PhD thesis, Stanford University, 2008. URL http://cdms.berkeley.edu/Dissertations/ogburn. pdf.

[105] W.W. Daniel. Applied nonparametric statistics. The Duxbury advanced series in statistics and decision sciences. PWS-Kent Publ., 1990. ISBN 9780534919764. URL https://books.google.ca/books? id $=0 \mathrm{hPvAAAAMAAJ}$.

[106] K. Schneck. Low-frequency noise cut using Prodv5-3 PTlfnoise1OF quantities. CDMS Intern. Run 133-135 Anal. Ebook, 10HT, 2013. URL http://titus.stanford.edu/cdms_restricted/Soudan/ R133/ebook/130605/index.html. 
[107] Yoav Freund and Robert E Schapire. A decision-theoretic generalization of on-line learning and an application to boosting. J. Comput. Syst. Sci., 55(1):119-139, August 1997. ISSN 0022-0000. doi: 10.1006/ jcss.1997.1504. URL http://dx.doi.org/10.1006/jcss.1997.1504.

[108] Hui Zou, J Zhu, S Rosset, and T Hastie. Multi-class adaboost. Statistics and its Interface, 2:349-360, 2009. ISSN 1938-7989.

[109] C. Gini. On the measure of concentration with special reference to income and statistics. Colorado College Publication, 208(9):73-79, 1936.

[110] J. Nix, J. Ma, G.N. Perdue, Y. Zheng, and Y.W. Wah. Blind background prediction using a bifurcated analysis scheme. Nucl. Instrum. Methods Phys. Res. A, 615(2):223-229, 2010. ISSN 0168-9002. doi: https://doi.org/10.1016/j.nima.2010.01.074. URL http://www . sciencedirect.com/science/article/pii/S0168900210001683.

[111] Vadim Liviu Rusu. PhD thesis, University of Pennsylvania, 2003. URL https://sno.phy.queensu.ca/papers/VadimRusuPhD.pdf.

[112] Hill Peter D. Kernel estimation of a distribution function. Communications in Statistics - Theory and Methods, 14(3):605-620, 1985. doi: 10.1080/03610928508828937. URL https://doi.org/10.1080/ 03610928508828937.

[113] D. Barker. Resolution Model for Simulation Data . CDMS Intern. Backgrounds Ebook, 2017. URL http://titus.stanford.edu/ cdms_restricted/barker/LowEnergyBkgdModel/SurfaceBkgd/ 14Dec2017.html.

[114] R. Underwood. CDMSlite Run 3 Radial Resolution. CDMS Intern. Run 133-135 Anal. Ebook, 56lite3, 2018. URL http://titus. stanford.edu/cdms_restricted/Soudan/R133/ebook/180130_RU/ PeakEfficiencyR3/QueensCDMSlite_peak_efficiency.html.

[115] W. Page. CDMSlite R3 Phonon Energy Calibration and Correction . CDMS Intern. Run 133-135 Anal. Ebook, 10lite3, 2016. URL http://titus.stanford.edu/cdms_restricted/Soudan/R133/ ebook/160613_BP/lite3GainCorrect.html.

[116] W. Page. Optimum Interval Sensitivity vs. Radial Cut Location. CDMS Intern. Run 133-135 Anal. Ebook, 72lite3, 2018. URL http://titus.stanford.edu/cdms_restricted/Soudan/R133/ ebook/180427_BP/R3RadialCutOptimization.html. 
[117] S. Hertel. R133 detector summary. CDMS Intern. Run 133-135 Anal. Ebook, HT1, 2012. URL http://titus.stanford.edu/cdms_ restricted/Soudan/R133/ebook/r133detectors.html.

[118] W. Page. CDMSlite R3 Trigger Efficiency. CDMS Intern. Run 133-135 Anal. Ebook, 11lite3, $2016 . \quad$ URL http://titus.stanford.edu/cdms_restricted/Soudan/R133/ ebook/160913_BP/R3TrigEff.html.

[119] Daniel Foreman-Mackey, David W. Hogg, Dustin Lang, and Jonathan Goodman. emcee : The mcmc hammer. Publications of the Astronomical Society of the Pacific, 125(925):306, 2013. URL http: //stacks. iop.org/1538-3873/125/i=925/a=306.

[120] L. Hsu A.J. Anderson and B. Serfass. Estimation of quality cut efficiencies from template-based pulse simulation . CDMS Intern. Run 133-135 Anal. Ebook, 38LT, 2013. URL http://titus.stanford. edu/cdms_restricted/Soudan/R133/ebook/131010/.

[121] Z. XU T. Ullrich. Treatment of Errors in Efficiency Calculations. arXiv:0701199, 2007. URL https://arxiv.org/pdf/physics/ 0701199v1.pdf.

[122] R. Underwood K. Page. Calculating $10 \mathrm{keV}$ line efficiency and the energy-independent part of efficiency of the radial cut in CDMSlite. CDMS Intern. Run 133-135 Anal. Ebook, 68lite2, 2015. URL http://titus.stanford.edu/cdms_restricted/Soudan/ R133/ebook/150731_kmp/150730_kmp/QueensCDMSlite_energy_ efficiency.html.

[123] D. Barker. SuperCDMS Background Models for Low-Mass Dark Matter Searches. PhD thesis, University of Minnesota, 2018. URL http://cdms . berkeley . edu/Dissertations/barker . pdf.

[124] E. Lopez Asamar D. Barker. Prediction of the radiogenic neutron background in CDMSlite run 3. CDMS Intern. Backgrounds Ebook, 18-lite2, 2017. URL http://titus.stanford.edu/cdms_ restricted/Soudan/R133/ebook/170904_ELA/index.html.

[125] U. Fano. Ionization Yield of Radiations. II. The Fluctuations of the Number of Ions. Phys. Rev., 72(1):26-29, jul 1947. ISSN 0031-899X. doi: 10.1103/PhysRev.72.26. URL https://link.aps.org/doi/10. 1103/PhysRev.72.26. 
[126] T. Aramaki I.J. Arnquist E. Azadbakht W. Baker S. Banik D. Barker D.A. Bauer T. Binder M.A. Bowles P.L. Brink R. Bunker B. Cabrera R. Calkins R.A. Cameron C. Cartaro D.G. Cerdeo Y.-Y. Chang J. Cooley B. Cornell P. Cushman F. De Brienne T. Doughty E. Fascione E. Figueroa-Feliciano C.W. Fink M. Fritts G. Gerbier R. Germond M. Ghaith S.R. Golwala H.R. Harris N. Herbert Z. Hong E.W. Hoppe L. Hsu M.E. Huber V. Iyer D. Jardin A. Jastram C. Jena M.H. Kelsey A. Kennedy A. Kubik N.A. Kurinsky R.E. Lawrence B. Loer E. Lopez Asamar P. Lukens D. MacDonell R. Mahapatra V. Mandic N. Mast E. Miller N. Mirabolfathi B. Mohanty J.D. Morales Mendoza J. Nelson H. Neog J.L. Orrell S.M. Oser W.A. Page R. Partridge M. Pepin F. Ponce S. Poudel M. Pyle H. Qiu W. Rau A. Reisetter R. Ren T. Reynolds A. Roberts A.E. Robinson H.E. Rogers T. Saab B. Sadoulet J. Sander A. Scarff R.W. Schnee S. Scorza K. Senapati B. Serfass D. Speller C. Stanford M. Stein J. Street H.A. Tanaka D. Toback R. Underwood A.N. Villano B. von Krosigk S.L. Watkins J.S. Wilson M.J. Wilson J. Winchell D.H. Wright S. Yellin B.A. Young X. Zhang X. Zhao R. Agnese, T. Aralis. Search for Low-Mass Dark Matter with CDMSlite Using a Profile Likelihood Fit. Submitted for publication in Physical Review D Journal, 2018. URL https://arxiv.org/abs/ 1808.09098.

[127] R. Agnese, T. Aralis, T. Aramaki, I. J. Arnquist, E. Azadbakht, W. Baker, D. Barker, D. A. Bauer, T. Binder, M. A. Bowles, P. L. Brink, R. Bunker, B. Cabrera, R. Calkins, C. Cartaro, D. G. Cerdeño, Y. Y. Chang, J. Cooley, B. Cornell, P. Cushman, T. Doughty, E. Fascione, E. Figueroa-Feliciano, C. W. Fink, M. Fritts, G. Gerbier, R. Germond, M. Ghaith, S. R. Golwal, H. R. Harris, Z. Hong, E. W. Hoppe, L. Hsu, M. E. Huber, V. Iyer, D. Jardin, A. Jastram, C. Jena, M. H. Kelsey, A. Kennedy, A. Kubik, N. A. Kurinsky, R. E. Lawrence, B. Loer, E. Lopez Asamar, P. Lukens, D. MacDonell, R. Mahapatra, V. Mandic, N. Mast, E. Miller, N. Mirabolfathi, B. Mohanty, J. D. Morales Mendoza, J. Nelson, J. L. Orrell, S. M. Oser, W. A. Page, R. Partridge, M. Pepin, F. Ponce, S. Poudel, M. Pyle, H. Qiu, W. Rau, A. Reisetter, R. Ren, T. Reynolds, A. Roberts, A. E. Robinson, H. E. Rogers, T. Saab, B. Sadoulet, S. Banik, J. Sander, A. Scarff, R. W. Schnee, S. Scorza, K. Senapati, B. Serfass, D. Speller, M. Stein, J. Street, H. A. Tanaka, D. Toback, R. Underwood, A. N. Villano, B. von Krosigk, S. L. Watkins, J. S. Wilson, M. J. Wilson, J. Winchell, D. H. Wright, S. Yellin, 
B. A. Young, X. Zhang, and X. Zhao. Production Rate Measurement of Tritium and Other Cosmogenic Isotopes in Germanium with CDMSlite. jun 2018. doi: 10.1016/j.astropartphys.2018.08.006. URL http://arxiv.org/abs/1806.07043. accepted for publication in Astropart. Phys.

[128] Kenneth S. Krane. Introductory Nuclear Physics. John Wiley \& Sons, Inc, 2nd edition, 1988. ISBN 978-0471805533.

[129] Bogdan Povh, Klaus Rith, Christoph Scholz, and Frank Zetsche. Particles and Nuclei: An Introduction to the Physical Concepts. Springer, 2008. ISBN 978-3-540-79367-0.

[130] Albert Thompson, David Attwood, Eric Gullikson, Malcolm Howells, Kwang-Je Kim, Janos Kirz, Jeffrey Kortright, Ingolf Lindau, Yanwei Liu, Piero Pianetta, Arthur Robinson, James Scofield, James Underwood, Gwyn Williams, and Herman Winick. X-Ray Data Booklet. Technical report, Lawrence Berkeley National Laboratory, Berkeley, 2009. URL http://xdb.1bl.gov/.

[131] E. Schönfeld. Calculation of fractional electron capture probabilities. Appl. Radiat. Isot., 49(9-11):1353-1357, aug 1998. ISSN 0969-8043. doi: 10.1016/S0969-8043(97)10073-2. URL https://www.sciencedirect.com/science/article/pii/ S0969804397100732?via\{\%\}3Dihub.

[132] J.M.C. Brown, M.R. Dimmock, J.E. Gillam, and D.M. Paganin. A low energy bound atomic electron Compton scattering model for Geant4. Nucl. Instrum. Methods Phys. Res. B, 338:77-88, 2014. doi: 10.1016/ j.nimb.2014.07.042.

[133] K. Ramanathan, A. Kavner, A. E. Chavarria, P. Privitera, D. Amidei, T.-L. Chou, A. Matalon, R. Thomas, J. Estrada, J. Tiffenberg, and J. Molina. Measurement of low energy ionization signals from Compton scattering in a charge-coupled device dark matter detector. Phys. Rev. D, 96(4):042002, aug 2017. ISSN 2470-0010. doi: 10.1103/PhysRevD.96.042002. URL https://link.aps.org/doi/ 10.1103/PhysRevD.96.042002.

[134] D'Ann Barker. Low Energy Background Spectrum in CDMSlite. In Proc. 38th Int. Conf. High Energy Phys., volume 282, page 874, Chicago, IL, feb 2017. doi: 10.22323/1.282.0874. URL https: //pos.sissa.it/282/874. 
[135] S. Agostinelli, J. Allison, K. Amako, J. Apostolakis, H. Araujo, P. Arce, M. Asai, D. Axen, S. Banerjee, G. Barrand, F. Behner, L. Bellagamba, J. Boudreau, L. Broglia, A. Brunengo, H. Burkhardt, S. Chauvie, J. Chuma, R. Chytracek, G. Cooperman, G. Cosmo, P. Degtyarenko, A. Dell'Acqua, G. Depaola, D. Dietrich, R. Enami, A. Feliciello, C. Ferguson, H. Fesefeldt, G. Folger, F. Foppiano, A. Forti, S. Garelli, S. Giani, R. Giannitrapani, D. Gibin, J.J. Gómez Cadenas, I. González, G. Gracia Abril, G. Greeniaus, W. Greiner, V. Grichine, A. Grossheim, S. Guatelli, P. Gumplinger, R. Hamatsu, K. Hashimoto, H. Hasui, A. Heikkinen, A. Howard, V. Ivanchenko, A. Johnson, F.W. Jones, J. Kallenbach, N. Kanaya, M. Kawabata, Y. Kawabata, M. Kawaguti, S. Kelner, P. Kent, A. Kimura, T. Kodama, R. Kokoulin, M. Kossov, H. Kurashige, E. Lamanna, T. Lampén, V. Lara, V. Lefebure, F. Lei, M. Liendl, W. Lockman, F. Longo, S. Magni, M. Maire, E. Medernach, K. Minamimoto, P. Mora de Freitas, Y. Morita, K. Murakami, M. Nagamatu, R. Nartallo, P. Nieminen, T. Nishimura, K. Ohtsubo, M. Okamura, S. O'Neale, Y. Oohata, K. Paech, J. Perl, A. Pfeiffer, M.G. Pia, F. Ranjard, A. Rybin, S. Sadilov, E. Di Salvo, G. Santin, T. Sasaki, N. Savvas, Y. Sawada, S. Scherer, S. Sei, V. Sirotenko, D. Smith, N. Starkov, H. Stoecker, J. Sulkimo, M. Takahata, S. Tanaka, E. Tcherniaev, E. Safai Tehrani, M. Tropeano, P. Truscott, H. Uno, L. Urban, P. Urban, M. Verderi, A. Walkden, W. Wander, H. Weber, J.P. Wellisch, T. Wenaus, D.C. Williams, D. Wright, T. Yamada, H. Yoshida, and D. Zschiesche. Geant4 - a simulation toolkit. Nucl. Instrum. Methods Phys. Res. A, 506(3):250-303, jul 2003. ISSN 0168-9002. doi: 10.1016/S0168-9002(03)01368-8. URL http://www . sciencedirect.com/science/article/pii/S0168900203013688.

[136] J. Allison, K. Amako, J. Apostolakis, H. Araujo, P. Arce Dubois, M. Asai, G. Barrand, R. Capra, S. Chauvie, R. Chytracek, G.A.P. Cirrone, G. Cooperman, G. Cosmo, G. Cuttone, G.G. Daquino, M. Donszelmann, M. Dressel, G. Folger, F. Foppiano, J. Generowicz, V. Grichine, S. Guatelli, P. Gumplinger, A. Heikkinen, I. Hrivnacova, A. Howard, S. Incerti, V. Ivanchenko, T. Johnson, F. Jones, T. Koi, R. Kokoulin, M. Kossov, H. Kurashige, V. Lara, S. Larsson, F. Lei, O. Link, F. Longo, M. Maire, A. Mantero, B. Mascialino, I. McLaren, P. Mendez Lorenzo, K. Minamimoto, K. Murakami, P. Nieminen, L. Pandola, S. Parlati, L. Peralta, J. Perl, A. Pfeiffer, M.G. Pia, A. Ribon, P. Rodrigues, G. Russo, S. Sadilov, 
G. Santin, T. Sasaki, D. Smith, N. Starkov, S. Tanaka, E. Tcherniaev, B. Tome, A. Trindade, P. Truscott, L. Urban, M. Verderi, A. Walkden, J.P. Wellisch, D.C. Williams, D. Wright, and H. Yoshida. Geant4 developments and applications. IEEE Trans. Nucl. Sci., 53(1):270278, feb 2006. ISSN 0018-9499. doi: 10.1109/TNS.2006.869826. URL http://ieeexplore.ieee.org/document/1610988/.

[137] J. Allison, K. Amako, J. Apostolakis, P. Arce, M. Asai, T. Aso, E. Bagli, A. Bagulya, S. Banerjee, G. Barrand, B.R. Beck, A.G. Bogdanov, D. Brandt, J.M.C. Brown, H. Burkhardt, Ph. Canal, D. CanoOtt, S. Chauvie, K. Cho, G.A.P. Cirrone, G. Cooperman, M.A. CortésGiraldo, G. Cosmo, G. Cuttone, G. Depaola, L. Desorgher, X. Dong, A. Dotti, V.D. Elvira, G. Folger, Z. Francis, A. Galoyan, L. Garnier, M. Gayer, K.L. Genser, V.M. Grichine, S. Guatelli, P. Guèye, P. Gumplinger, A.S. Howard, I. Hivnáčová, S. Hwang, S. Incerti, A. Ivanchenko, V.N. Ivanchenko, F.W. Jones, S.Y. Jun, P. Kaitaniemi, N. Karakatsanis, M. Karamitros, M. Kelsey, A. Kimura, T. Koi, H. Kurashige, A. Lechner, S.B. Lee, F. Longo, M. Maire, D. Mancusi, A. Mantero, E. Mendoza, B. Morgan, K. Murakami, T. Nikitina, L. Pandola, P. Paprocki, J. Perl, I. Petrović, M.G. Pia, W. Pokorski, J.M. Quesada, M. Raine, M.A. Reis, A. Ribon, A. Ristić Fira, F. Romano, G. Russo, G. Santin, T. Sasaki, D. Sawkey, J.I. Shin, I.I. Strakovsky, A. Taborda, S. Tanaka, B. Tomé, T. Toshito, H.N. Tran, P.R. Truscott, L. Urban, V. Uzhinsky, J.M. Verbeke, M. Verderi, B.L. Wendt, H. Wenzel, D.H. Wright, D.M. Wright, T. Yamashita, J. Yarba, and H. Yoshida. Recent developments in Geant4. Nucl. Instrum. Methods Phys. Res. A, 835:186-225, nov 2016. ISSN 0168-9002. doi: 10.1016/J.NIMA.2016.06.125. URL http://www . sciencedirect.com/science/article/pii/S0168900216306957.

[138] Marcus H. Mendenhall and Robert A. Weller. An algorithm for computing screened Coulomb scattering in Geant4. Nucl. Instrum. Methods Phys. Res. B, 227(3):420-430, 2005. ISSN 0168583X. doi: 10.1016/j.nimb.2004.08.014. URL http://www.sciencedirect.com/ science/article/pii/S0168583X04009851.

[139] E. Aprile, K. Arisaka, F. Arneodo, A. Askin, L. Baudis, A. Behrens, K. Bokeloh, E. Brown, T. Bruch, J. M. R. Cardoso, B. Choi, D. Cline, E. Duchovni, S. Fattori, A. D. Ferella, K.-L. Giboni, E. Gross, A. Kish, C. W. Lam, J. Lamblin, R. F. Lang, K. E. Lim, S. Lindemann, M. Lindner, J. A. M. Lopes, T. Marrodán Undagoitia, Y. Mei, A. J. 
Melgarejo Fernandez, K. Ni, U. Oberlack, S. E. A. Orrigo, E. Pantic, G. Plante, A. C. C. Ribeiro, R. Santorelli, J. M. F. dos Santos, M. Schumann, P. Shagin, A. Teymourian, D. Thers, E. Tziaferi, O. Vitells, H. Wang, M. Weber, and C. Weinheimer. Likelihood approach to the first dark matter results from XENON100. Phys. Rev. D, 84:052003, Sep 2011. doi: 10.1103/PhysRevD.84.052003. URL https://link.aps.org/doi/10.1103/PhysRevD.84.052003.

[140] S. S. Wilks. The Large-Sample Distribution of the Likelihood Ratio for Testing Composite Hypotheses. Ann. Math. Statist., 9(1):60-62, 03 1938. doi: 10.1214/aoms/1177732360. URL https://doi .org/10. 1214/aoms/1177732360.

[141] A. L. Read. Presentation of search results: The $\mathrm{CL}_{s}$ technique. J. Phys. G, 28(10):2693, 2002. URL http://stacks.iop.org/ $0954-3899 / 28 / i=10 / a=313$.

[142] Z. W. Birnbaum. Distribution-free Tests of fit for Continuous Distribution Functions. Ann. Math. Statist., 24(1):1-8, 03 1953. doi: 10.1214/aoms/1177729078. URL https://doi.org/10.1214/aoms/ 1177729078.

[143] R. Agnese, A. J. Anderson, T. Aramaki, M. Asai, W. Baker, D. Balakishiyeva, D. Barker, R. Basu Thakur, D. A. Bauer, J. Billard, A. Borgland, M. A. Bowles, P. L. Brink, R. Bunker, B. Cabrera, D. O. Caldwell, R. Calkins, D. G. Cerdeno, H. Chagani, Y. Chen, J. Cooley, B. Cornell, P. Cushman, M. Daal, P. C. F. Di Stefano, T. Doughty, L. Esteban, S. Fallows, E. Figueroa-Feliciano, M. Ghaith, G. L. Godfrey, S. R. Golwala, J. Hall, H. R. Harris, T. Hofer, D. Holmgren, L. Hsu, M. E. Huber, D. Jardin, A. Jastram, O. Kamaev, B. Kara, M. H. Kelsey, A. Kennedy, A. Leder, B. Loer, E. Lopez Asamar, P. Lukens, R. Mahapatra, V. Mandic, N. Mast, N. Mirabolfathi, R. A. Moffatt, J. D. Morales Mendoza, S. M. Oser, K. Page, W. A. Page, R. Partridge, M. Pepin, A. Phipps, K. Prasad, M. Pyle, H. Qiu, W. Rau, P. Redl, A. Reisetter, Y. Ricci, A. Roberts, H. E. Rogers, T. Saab, B. Sadoulet, J. Sander, K. Schneck, R. W. Schnee, S. Scorza, B. Serfass, B. Shank, D. Speller, D. Toback, R. Underwood, S. Upadhyayula, A. N. Villano, B. Welliver, J. S. Wilson, D. H. Wright, S. Yellin, J. J. Yen, B. A. Young, and J. Zhang. New Results from the Search for Low-Mass Weakly Interacting Massive Particles with the CDMS Low Ionization Threshold Experiment. 
Phys. Rev. Lett., 116(7):071301, feb 2016. ISSN 0031-9007. doi: 10.1103/PhysRevLett.116.071301. URL http://link.aps.org/doi/ 10.1103/PhysRevLett.116.071301.

[144] C. Amole, M. Ardid, I. J. Arnquist, D. M. Asner, D. Baxter, E. Behnke, P. Bhattacharjee, H. Borsodi, M. Bou-Cabo, P. Campion, G. Cao, C. J. Chen, U. Chowdhury, K. Clark, J. I. Collar, P. S. Cooper, M. Crisler, G. Crowder, C. E. Dahl, M. Das, S. Fallows, J. Farine, I. Felis, R. Filgas, F. Girard, G. Giroux, J. Hall, O. Harris, E. W. Hoppe, M. Jin, C. B. Krauss, M. Laurin, I. Lawson, A. Leblanc, I. Levine, W. H. Lippincott, F. Mamedov, D. Maurya, P. Mitra, T. Nania, R. Neilson, A. J. Noble, S. Olson, A. Ortega, A. Plante, R. Podviyanuk, S. Priya, A. E. Robinson, A. Roeder, R. Rucinski, O. Scallon, S. Seth, A. Sonnenschein, N. Starinski, I. Štekl, F. Tardif, E. Vázquez-Jáuregui, J. Wells, U. Wichoski, Y. Yan, V. Zacek, and J. Zhang. Dark Matter Search Results from the PICO-60 $\mathrm{C}_{3} \mathrm{~F}_{8}$ Bubble Chamber. Phys. Rev. Lett., 118:251301, Jun 2017. doi: 10.1103/PhysRevLett.118.251301. URL https://link.aps.org/doi/10.1103/PhysRevLett.118.251301.

[145] H. Jiang, L. P. Jia, Q. Yue, K. J. Kang, J. P. Cheng, Y. J. Li, H. T. Wong, M. Agartioglu, H. P. An, J. P. Chang, J. H. Chen, Y. H. Chen, Z. Deng, Q. Du, H. Gong, L. He, J. W. Hu, Q. D. Hu, H. X. Huang, H. B. Li, H. Li, J. M. Li, J. Li, X. Li, X. Q. Li, Y. L. Li, B. Liao, F. K. Lin, S. T. Lin, S. K. Liu, Y. D. Liu, Y. Y. Liu, Z. Z. Liu, H. Ma, J. L. Ma, H. Pan, J. Ren, X. C. Ruan, B. Sevda, V. Sharma, M. B. Shen, L. Singh, M. K. Singh, T. X. Sun, C. J. Tang, W. Y. Tang, Y. Tian, G. F. Wang, J. M. Wang, L. Wang, Q. Wang, Y. Wang, S. Y. Wu, Y. C. Wu, H. Y. Xing, Y. Xu, T. Xue, L. T. Yang, S. W. Yang, N. Yi, C. X. Yu, H. J. Yu, J. F. Yue, X. H. Zeng, M. Zeng, Z. Zeng, F. S. Zhang, Y. H. Zhang, M. G. Zhao, J. F. Zhou, Z. Y. Zhou, J. J. Zhu, and Z. H. Zhu. Limits on Light Weakly Interacting Massive Particles from the First $102.8 \mathrm{~kg} \times$ day Data of the CDEX-10 Experiment. Phys. Rev. Lett., 120:241301, Jun 2018. doi: 10.1103/PhysRevLett.120.241301. URL https://link.aps.org/doi/10.1103/PhysRevLett.120.241301.

[146] Noah Kurinsky, Paul Brink, Richard Partridge, Blas Cabrera, and Matt Pyle. SuperCDMS SNOLAB Low-Mass Detectors: UltraSensitive Phonon Calorimeters for a Sub-GeV Dark Matter Search. 
In Proc. 38th Int. Conf. High Energy Phys., Chicago, IL, nov 2016. URL http://arxiv.org/abs/1611.04083.

[147] D. S. Akerib, H. M. Araújo, X. Bai, A. J. Bailey, J. Balajthy, P. Beltrame, E. P. Bernard, A. Bernstein, T. P. Biesiadzinski, E. M. Boulton, A. Bradley, R. Bramante, S. B. Cahn, M. C. Carmona-Benitez, C. Chan, J. J. Chapman, A. A. Chiller, C. Chiller, A. Currie, J. E. Cutter, T. J. R. Davison, L. de Viveiros, A. Dobi, J. E. Y. Dobson, E. Druszkiewicz, B. N. Edwards, C. H. Faham, S. Fiorucci, R. J. Gaitskell, V. M. Gehman, C. Ghag, K. R. Gibson, M. G. D. Gilchriese, C. R. Hall, M. Hanhardt, S. J. Haselschwardt, S. A. Hertel, D. P. Hogan, M. Horn, D. Q. Huang, C. M. Ignarra, M. Ihm, R. G. Jacobsen, W. Ji, K. Kazkaz, D. Khaitan, R. Knoche, N. A. Larsen, C. Lee, B. G. Lenardo, K. T. Lesko, A. Lindote, M. I. Lopes, D. C. Malling, A. Manalaysay, R. L. Mannino, M. F. Marzioni, D. N. McKinsey, D.-M. Mei, J. Mock, M. Moongweluwan, J. A. Morad, A. St. J. Murphy, C. Nehrkorn, H. N. Nelson, F. Neves, K. O’Sullivan, K. C. Oliver-Mallory, R. A. Ott, K. J. Palladino, M. Pangilinan, E. K. Pease, P. Phelps, L. Reichhart, C. Rhyne, S. Shaw, T. A. Shutt, C. Silva, V. N. Solovov, P. Sorensen, S. Stephenson, T. J. Sumner, M. Szydagis, D. J. Taylor, W. Taylor, B. P. Tennyson, P. A. Terman, D. R. Tiedt, W. H. To, M. Tripathi, L. Tvrznikova, S. Uvarov, J. R. Verbus, R. C. Webb, J. T. White, T. J. Whitis, M. S. Witherell, F. L. H. Wolfs, K. Yazdani, S. K. Young, and C. Zhang. Improved limits on scattering of weakly interacting massive particles from reanalysis of 2013 lux data. Phys. Rev. Lett., 116:161301, Apr 2016. doi: 10.1103/PhysRevLett.116.161301. URL https://link.aps.org/doi/10.1103/PhysRevLett.116.161301.

[148] F. Ruppin, J. Billard, E. Figueroa-Feliciano, and L. Strigari. Complementarity of dark matter detectors in light of the neutrino background. Phys. Rev. D, 90:083510, Oct 2014. doi: 10.1103/PhysRevD. 90.083510. URL https://link.aps.org/doi/10.1103/PhysRevD. 90.083510.

[149] A. Phipps. Ionization Collection in Detectors of the Cryogenic Dark Matter Search. PhD thesis, University of California, Berkeley, 2016. URL http://cdms.berkeley.edu/Dissertations/phipps.pdf. 


\section{Appendix A}

\section{Voltage Scan Calibration Results}

\section{A.1 Linearity}

Calibration studies from CDMSlite Run 1 and Run 2 indicated that the energy scale of the CDMSlite detectors was more complex than the simple Neganov-Trofimov-Luke model (Eq. 2.9) and that the total phonon amplification was not exactly linear as a function of bias voltage, $V_{\mathrm{b}}$. These studies also indicated that the ionization and phonon collection of the CDMSlite detectors differed from the iZIP detectors and therefore the energy scale differed between the two detector configurations.

Therefore, prior to the CDMSlite Run 3 analysis, we performed a dedicated study of the CDMSlite detectors' energy scale as a function of bias voltage. The $10.4 \mathrm{keV}_{\text {ee }}$ and $1.3 \mathrm{keV}_{\text {ee }}$ ER events were used to calibrate the energy scale of both the T2Z1 and T5Z2 detectors at a variety of voltages. When first processing the data, the calibration used was the iZIP calibration which was determined with the detector at 4V. Fig. 2.14 and A.1 show the reconstructed energy of the $10.4 \mathrm{keV}_{\text {ee }}$ and $1.3 \mathrm{keV}_{\text {ee }}$ peaks for T5Z2 detector voltages of $0 \mathrm{~V}, 30 \mathrm{~V}, 50 \mathrm{~V}$, and $70 \mathrm{~V}$.

\section{A.2 Significance of Calibration Systematics}

The bias scan data can be better explained with slight modifications to simple Luke gain scaling assumption: $E_{p t}=E_{r}\left(1+\frac{e V_{b}}{\epsilon}\right)$. If there is suppressed collection of recombination phonons in CDMSlite, but we still assume 100\% collection of recombination phonons, how does this affect the ER and NR energy scale? If there is impact ionization ${ }^{10}$ that is ignored, how does this

\footnotetext{
${ }^{10}$ Impact ionization is a process by which $e^{-} / h^{+}$pairs in the detectors can ionize additional $e^{-} / h^{+}$pairs, thereby creating additional Luke phonons with an energy that is non-linear as a function of the voltage across the detector. While this is a second-order process, as seen by the fact that the total phonon energy scale is to first order linear with
} 


\section{A.2. Significance of Calibration Systematics}
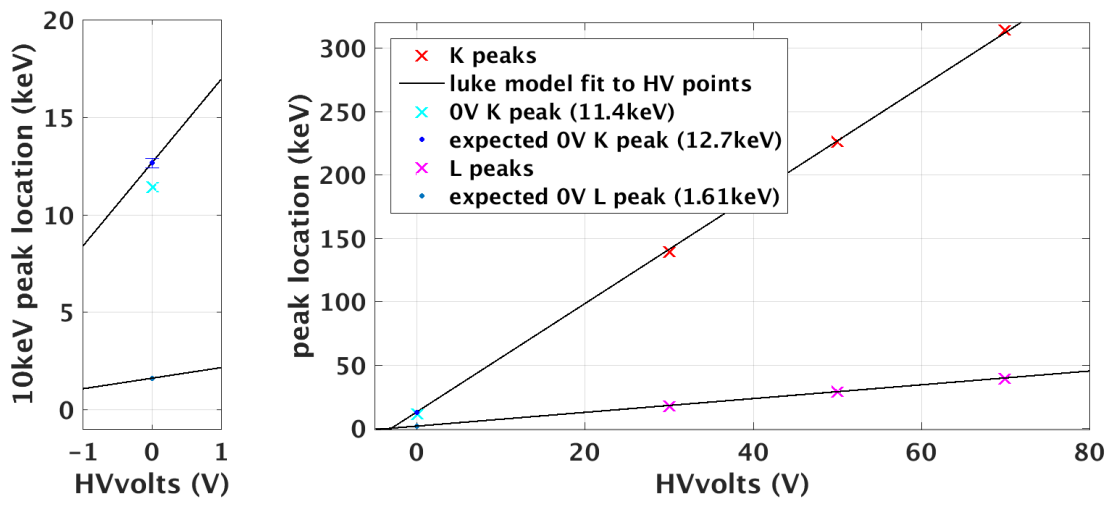

Figure A.1: (left) The zoomed-in version of the left hand plot highlights the discrepancy between the expected, by extrapolation, location of the $K$-shell peak at $0 \mathrm{~V}$. (right) The location of the $K$ - and $L$ - shell peaks as a function of detector voltage. The higher voltage peaks $(30 \mathrm{~V}, 50 \mathrm{~V}$, and $70 \mathrm{~V})$ are linear to within uncertainty, but there is a deviation from linearity at $0 \mathrm{~V}$. The overall energy scale (y axis) has used a calibration constant for the detector obtained at $4 \mathrm{~V}$, though the overall scaling of the $\mathrm{y}$ axis is irrelevant when checking for linearity.

affect the ER and NR energy scale? We now introduce a few variables to model these effects. The true recombination phonon collection fraction is given by $f_{R C, T}$. The true Luke phonon collection fraction is given by $f_{L, T}$. Fortunately, neglecting the two effects above will have no effect on the electron recoil energy scale, as discussed below. In the NR energy scale, neglecting the effects will only introduce a slight bias. The plots in Fig. A.2 show the ratio between the measured NR energy, $E_{n r, M}$, and the true NR energy, $E_{n r, T}$, as a function of $f_{R C, T}$ and $f_{L, T}$. We used an example ionization yield of $Y\left(E_{r}\right)=0.2$ and assumed $\epsilon=3 \mathrm{eV}$.

\section{Electron Recoils}

Due to the ratio of $E_{\text {gap }}=0.79 \mathrm{eV}$ to $E_{\text {create }}=3 \mathrm{eV}$ in $\mathrm{Ge}, 74 \%$ of an electron recoil's energy, $E_{r}$, goes into primary phonons. The other $26 \%$ of energy goes into ionization, which either recombines immediately, emitting the energy as phonons (when $V_{b}=0$ ), or drifts across the crystal emitting energy as Luke phonons $\left(E_{\text {luke }}=n_{e h} e V_{b}\right)$, until the ionization recombines

the voltage, it has been observed in CDMS style detectors[149]. 


\section{A.2. Significance of Calibration Systematics}

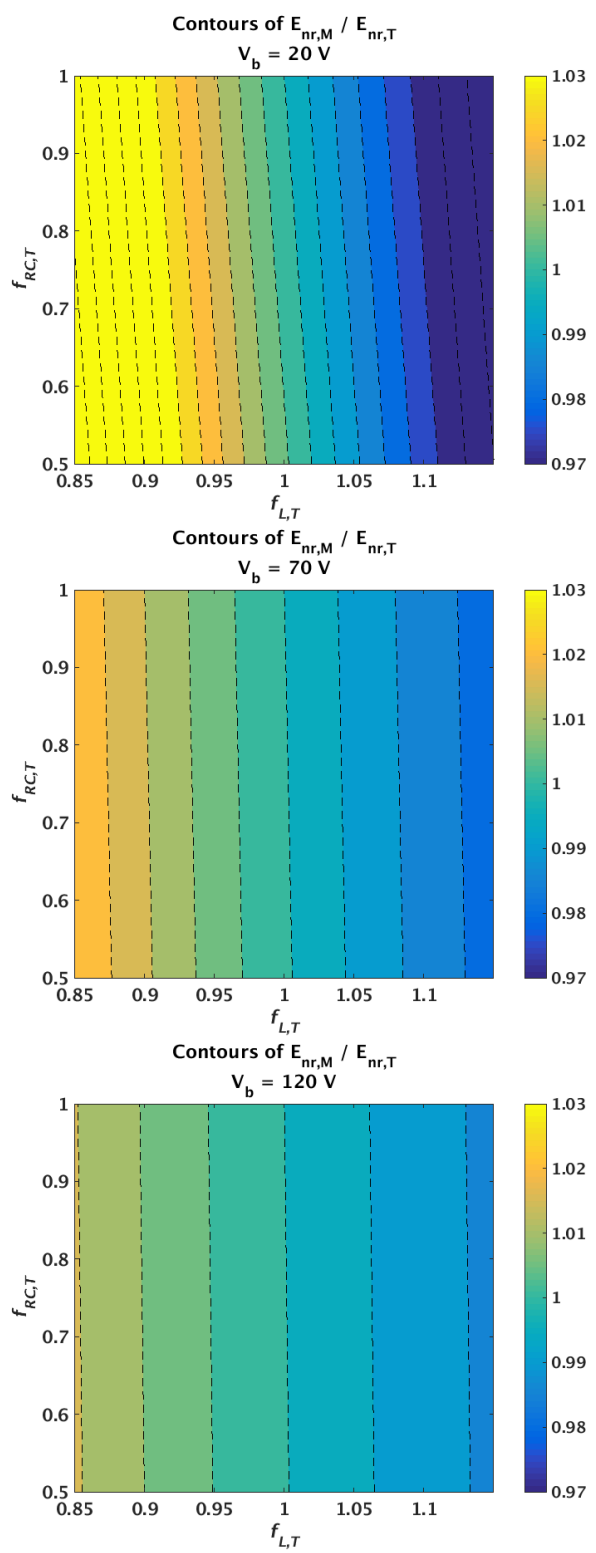

Figure A.2: Large systematics in Luke phonon collection (15\%) and recombination phonon collection $(50 \%)$ would contribute at most to a $\sim 3 \%$ error in the nuclear recoil energy scale. The recombination phonon error becomes negligible at high detector bias. Also the error decreases at higher detector voltage, $V_{b}$, as the total phonon energy becomes more correlated with the ionization. 


\section{A.2. Significance of Calibration Systematics}

at the electrodes and emits the recombination energy as phonons back into the crystal.

The bias scan data can be better explained with slight modifications to the above process. One explanation is that recombination phonons are never being collected. At $0 \mathrm{~V}$ charges could be trapping and never recombining. At higher voltages it's possible that recombination phonons are not being collected because (1) phonons created at the electrodes aren't emitted back into the crystal or (2) perhaps the charges trap near the surface and never recombine. We can treat this as a recombination phonon suppression, $f_{R C, T}$, which is $<1$.

An equally consistent explanation of the bias scan data is that impact ionization is occurring at the higher voltages, where drifting ionization deneutralizes an impurity, creating more ionization and therefore excess Luke phonons. We can treat this as a preferential collection of Luke phonons in the energy scaling, where $f_{L}, T>1$.

These effects modify the total phonon energy scale. With full recombination phonon collection and without impact ionization the total phonon energy scale becomes

$$
E_{p t}=E_{r}\left(1+\frac{e V_{b}}{\epsilon}\right)
$$

while with suppressed recombination phonon collection and impact ionization it becomes

$$
E_{p t}=E_{r}\left(0.74+0.26 \times f_{R C}+f_{L} \frac{e V_{b}}{\epsilon}\right)
$$

At a certain voltage $V_{b}$, we measure a calibration constant, $a_{M}$, that scales an ADC value to the total phonon energy, $E_{p t} . a_{M}$ is typically estimated given an electron recoil $E_{r}$ of known energy and assuming the energy scale of Eq. A.1:

$$
\begin{gathered}
E_{p t, M}=a_{M} \mathrm{ADC}, \\
a_{M}=\frac{10 \mathrm{keV}\left(1+\frac{e V_{b}}{\epsilon}\right)}{\left.\mathrm{ADC}\right|_{10 \mathrm{keV}}} .
\end{gathered}
$$

If Eq. A.1 is an incorrect energy scale, and instead the true energy scale is given by Eq. A.2, then the true calibration constant, $a_{T}$, would be different than $a_{M}$ (making $E_{p t, M}$ incorrect). The true calibration is denoted by 
subscript $T$ :

$$
a_{T}=\frac{10 \mathrm{keV}\left(0.74+0.26 \times f_{R C}+f_{L} \frac{e V_{b}}{\epsilon}\right)}{\left.\mathrm{ADC}\right|_{10 \mathrm{keV}}} .
$$

However, when converting from the total phonon energy scale to the electron recoil scale, if a mistake is done the calibration, that mistake is undone and the correct electron recoil energy is still obtained. Below we compare the measured (" $M$ ") electron recoil to the true (" $T$ ") electron recoil and see that they are equivalent:

$$
\begin{aligned}
E_{r, M} & =\frac{E_{p t, M}}{1+\frac{e V_{b}}{\epsilon}} \\
& =\frac{a_{M} \mathrm{ADC}}{1+\frac{e V_{b}}{\epsilon}} \\
& =\frac{10 \mathrm{keV}}{\left.\mathrm{ADC}\right|_{10 \mathrm{keV}}} \mathrm{ADC}
\end{aligned}
$$

vs.

$$
\begin{aligned}
E_{r, T} & =\frac{E_{p t, T}}{\left(0.74+0.26 \times f_{R C}+f_{L} \frac{e V_{b}}{\epsilon}\right)} \\
& =\frac{a_{T} \mathrm{ADC}}{\left(0.74+0.26 \times f_{R C}+f_{L} \frac{e V_{b}}{\epsilon}\right)} \\
& =\frac{10 \mathrm{keV}}{\left.\mathrm{ADC}\right|_{10 \mathrm{keV}}} \mathrm{ADC} .
\end{aligned}
$$

The bias in the $E_{p t, M}$ energy scale, is "calibrated out," and does not introduce a bias in the ER energy scale.

\section{Nuclear Recoils}

For the nuclear recoil energy scale a small $(\sim 1 \%)$ systematic is introduced when a wrong assumption is made about recombination phonon collection or impact ionization, which does not cancel out, because the CDMSlite calibration is always done using electron recoils. The full recombination phonon collection, without impact ionization, energy scale is given by

$$
E_{p t}=E_{n r}\left(1+Y \frac{e V_{b}}{\epsilon}\right)
$$




\section{A.2. Significance of Calibration Systematics}

while suppressed recombination phonon collection efficiency and impact ionization is given by

$$
E_{p t}=E_{n r}\left[(1-Y(0.26))+f_{R C} Y(0.26)+f_{L} Y \frac{e V_{b}}{\epsilon}\right] .
$$

When converting from total phonon energy to nuclear recoil energy, now the presence of the ionization yield factor $Y\left(E_{r}\right)$ creates the small systematic bias in the measured nuclear recoil $E_{n r, M}$ vs. the true nuclear recoil $E_{n r, T}$.

$$
\begin{gathered}
E_{n r, M}=\frac{E_{p t, M}}{1+Y \frac{e V_{b}}{\epsilon}}=\frac{a_{M} \times \mathrm{ADC}}{1+Y \frac{e V_{b}}{\epsilon}}=\frac{1}{1+Y \frac{e V_{b}}{\epsilon}} \frac{10 \mathrm{keV}\left(1+\frac{e V_{b}}{\epsilon}\right)}{\left.\mathrm{ADC}\right|_{10 \mathrm{keV}}} \times \mathrm{ADC} \\
E_{n r, T}=\frac{a_{T} \times \mathrm{ADC}}{(1-Y(0.26))+f_{R C} Y(0.26)+f_{L} Y \frac{e V_{b}}{\epsilon}} \\
=\frac{1}{(1-Y(0.26))+f_{R C} Y(0.26)+f_{L} Y \frac{e V_{b}}{\epsilon}} \frac{10 \mathrm{keV}\left(0.76+f_{L} \frac{e V_{b}}{\epsilon}\right)}{\left.\mathrm{ADC}\right|_{10 \mathrm{keV}}} \times \mathrm{ADC}
\end{gathered}
$$

The $a_{M}$ and $a_{T}$ are the calibrations from the Electron Recoil section above.

The ratio of the measured NR energy to the true NR energy is given by:

$$
\frac{E_{n r, M}}{E_{n r, T}}=\frac{\left[(1-Y(0.26))+f_{R C} Y(0.26)+f_{L} Y \frac{e V_{b}}{\epsilon}\right]\left[1+\frac{e V_{b}}{\epsilon}\right]}{\left[1+Y \frac{e V_{b}}{\epsilon}\right]\left[\left(0.74+0.26 \times f_{R C}+f_{L} \frac{e V_{b}}{\epsilon}\right)\right]} .
$$

The dependence of this ratio on $f_{L}$ and $f_{R C}$ is shown in the contour plots in Fig. A.2 at the beginning of this appendix. It shows that not accounting for suppressed recombination phonon collection and not accounting for impact ionization has a small effect on the NR energy scale.

Three different cases are shown explicitly here. We use a nuclear recoil with $Y=0.2$, a detector voltage bias of $70 \mathrm{~V}$, and assume $\epsilon=3 \mathrm{eV}$.

1. With no impact ionization, $f_{L}=1$, but with $f_{R C}=0$, the measured recoil energy is only $0.2 \%$ larger than the true recoil.

2. With $f_{L}=1.1$ (10\% impact ionization), and with $f_{R C}=0$ the measured recoil energy is $0.99 \%$ smaller than the true energy.

3. With $f_{L}=1.1$ and with $f_{R C}=1$, the measured recoil energy is $1.2 \%$ smaller than the true energy. 